Juan Carlos Cutipa Luque

\title{
Controle Robusto Multivariável para um Veículo Submersível Autônomo
}

Dissertação apresentada à Escola Politécnica da Universidade de São Paulo para obtenção do título de Mestre em Engenharia Mecânica. 


\section{Juan Carlos Cutipa Luque}

\section{Controle Robusto Multivariável para um Veículo Submersível Autônomo}

Dissertação apresentada à Escola Politécnica da Universidade de São Paulo para obtenção do título de Mestre em Engenharia Mecânica.

Área de concentração:

Engenharia de Controle e Automação Mecânica

Orientador:

Prof. Dr. Decio Crisol Donha 


\section{FICHA CATALOGRÁFICA}

\section{Cutipa Luque, Juan Carlos}

Controle robusto multivariável para um veículo submersível autônomo / J.C. Cutipa Luque. -- São Paulo, 2007.

$113 \mathrm{p}$.

Dissertação (Mestrado) - Escola Politécnica da Universidade de São Paulo. Departamento de Engenharia Mecânica.

1.Veículos submersíveis não tripulados 2.Sistemas de controle 3.Controle ótimo I.Universidade de São Paulo. Escola Politécnica. Departamento de Engenharia Mecânica Il.t. 
Aos meus pais, por tudo. 


\section{Agradecimentos}

Ao Prof. Decio Donha pela paciente orientação e pelo incentivo durante a realização deste trabalho.

Ao Prof. Jaime da Cruz, quem sempre esteve disposto a discutir minhas dúvidas referente ao controle em sistemas multivariáveis e assim dando a orientação certa ao trabalho.

À CAPES (Coordenação de Aperfeiçoamento de Pessoal de Nível Superior), pela concessão da bolsa de mestrado.

Ao Prof. Pablo Aco-Cárdenas, cuja motivação inicial, foi suficiente e necessária para continuar meus estudos de mestrado nesta prestigiosa Universidade.

Aos colegas dos Departamentos de Engenharia Mecânica e Engenharia Naval e Oceânica, e a todos aqueles que direta ou indiretamente colaboraram com a realização deste trabalho. 


\section{Resumo}

Este trabalho trata do controle dos movimentos de um Veículo Submersível Autônomo (VSA). Veículos submersíveis são difíceis de controlar devido à alta não linearidade de seus modelos, ao forte acoplamento de movimentos, ao desconhecimento de certas dinâmicas, às incertezas do próprio modelo, devido a distúrbios externos impostos pelo ambiente e devido ao ruído de sensores. A dificuldade de controle pode ser exacerbada quando o veículo é subatuado. Para realização deste trabalho foi escolhido um VSA do tipo torpedo, cujo modelo matemático disponível na literatura foi devidamente modificado para garantir uma melhor descrição de seus movimentos em seis graus de liberdade (6-GL). O modelo foi então validado através de simulações numéricas.

Para a síntese dos controladores utilizou-se uma técnica de controle avançada. Mais especificamente, utilizou-se a abordagem do controle $\mathcal{H}_{\infty}$ para sistemas multivariáveis. Assim foram obtidos controladores centralizados capazes de superar o problema do forte acoplamento de movimentos. Técnicas de controle avançado permitem também considerar as informações disponíveis sobre perturbações, incertezas, ruídos e diferentes tipos de entrada já na fase de síntese, o que permite obter controladores com desempenho adequado numa ampla faixa de operação. Neste trabalho, em particular, a técnica da Sensibilidade Mista foi escolhida para a síntese de controladores robustos. Nesta abordagem, formatam-se algumas funções de malha fechada ligadas a sensibilidade do sistema buscando garantir estabilidade e desempenho robusto para o sistema controlado. Usando a mesma técnica de controle desenvolveu-se ainda um controlador de dois graus de liberdade (2-GL), apropriado para aplicação no problema de guiagem, onde procura-se seguir trajetórias tridimensionais.

Os controladores desenvolvidos foram testados em simulações numéricas, produzindo-se uma grande quantidade de resultados. A análise destes resultados revela o poder e flexibilidade das técnicas escolhidas. 


\section{Abstract}

This work focuses the motion control of an Autonomous Underwater Vehicle (AUV). Underwater vehicles are difficult to control due to high non-linearities of its model, coupling between dynamics, unknown dynamics, model uncertainties, disturbances and sensor noises. Difficulty is greater, when the system is subactuated. In this work, a mathematical model of a torpedo-like AUV available in the bibliography was chosen and refined, leading to a six degree of freedom (6-DOF) model. The model was further analyzed and validated by a number of numerical simulations.

Advanced approaches were used for the synthesis of controllers. Specifically, a $\mathcal{H}_{\infty}$ approach for multivariable systems was used. Thus, a centralized controller was developed, able to avoid the problem of high coupling between the variables. This advanced approach is also able to use informations about perturbations, uncertainties, noises and different types of input signals in the synthesis stage, leading to controllers with better performance in a large operation bandwidth. In this work, a Mixed Sensitivity approach was employed. This control approach is based on the shapping of well known closed-loop sensitivity functions, seeking to achieve stability and performance robustness. Using a similar technique, a controller with two degree of freedom (2-DOF) was also synthesised, to tackle the guidance problem tracking of 3-D trajectories was then fully achieved.

The controllers developed were tested in a number of numerical simulations. Analyses of results reveals the power and flexibility of the employed techniques. 


\section{Sumário}

\section{Lista de Figuras}

Lista de Tabelas

Lista de Abreviaturas

Lista de Símbolos

1 Introdução 1

1.1 Motivação e Objetivos . . . . . . . . . . . . . . . 2

1.2 Revisão Bibliográfica . . . . . . . . . . . . . . . . . . 2

1.3 Estrutura da Dissertação . . . . . . . . . . . . . . . . . . 4

2 Descrição do Veículo 5

2.1 Características principais . . . . . . . . . . . . 5

2.2 Arranjo geral . . . . . . . . . . . . . . . . 7

2.3 Sensores de Navegação . . . . . . . . . . . . . . . . . . . . 8

2.4 Lastro . . . . . . . . . . . . . . . . . . . . . . . . . . . 9

2.5 Energia . . . . . . . . . . . . . . . . . . 9

2.6 Superfícies de Controle . . . . . . . . . . . . . . . . . . . 9

3 Modelagem Dinâmica do Veículo $\quad 11$

3.1 Introdução . . . . . . . . . . . . . . . . . . . . 11

3.2 Sistema de Coordenadas . . . . . . . . . . . . . . . . . . 11

3.3 Cinemática do Veículo . . . . . . . . . . . . . . . . . . . . . . . . 12 
3.4 Dinâmica de Corpo Rígido do Veículo . . . . . . . . . . . . . . . . . . . . . 13

3.4 .1 Movimentos de Translação . . . . . . . . . . . . . . . . . . . . 13

3.4 .2 Movimentos de Rotação . . . . . . . . . . . . . . . . . . 15

3.5 Coeficientes Hidrodinâmicos . . . . . . . . . . . . . . . . . . . . 17

3.5.1 Massa Adicionada . . . . . . . . . . . . . . . . . . . . . 18

3.5.2 Resistência ao Avanço . . . . . . . . . . . . . . . . . . . . . 19

3.5.3 Propulsor ............................ 20

3.5.4 Esforços de Sustentação . . . . . . . . . . . . . . . 22

3.5.5 Esforços Restauradores . . . . . . . . . . . . . . . . 24

3.6 Modelo Não Linear do Veículo . . . . . . . . . . . . . . . . . . . . . . . . . 24

3.7 Modelo Dinâmico Vetorial . . . . . . . . . . . . . . . . . . . . 26

3.8 Linearização . . . . . . . . . . . . . . . . . . . . . . . . . . . . . . 28

4 Lei de Controle $\quad 30$

4.1 Introdução . . . . . . . . . . . . . . . . . . . . 30

4.2 Abordagem de Projeto . . . . . . . . . . . . . . . . . . . 31

4.3 Representação do Sistema Dinâmico Linear . . . . . . . . . . . . . . . . . . 31

4.4 Controlabilidade e Observabilidade . . . . . . . . . . . . . . 33

4.5 Ferramentas Analíticas . . . . . . . . . . . . . . . . . 34

4.5.1 Valores Singulares . . . . . . . . . . . . . . . . . . 34

4.5.2 Norma $\mathcal{H}_{2}$ e $\mathcal{H}_{\infty} \ldots \ldots \ldots \ldots$. . . . . . . . . . . . . . . . . .

4.6 Incertezas . . . . . . . . . . . . . . . . . . . . 36

4.6.1 Incertezas Estruturadas . . . . . . . . . . . . . . . . . . 36

4.6 .2 Incertezas Sem-estrutura . . . . . . . . . . . . . . . . . . . 36

4.7 Especificações e Limitações de Desempenho . . . . . . . . . . . . . . . . . 38

4.7 .1 Normalização . . . . . . . . . . . . . . . . . . . . . . . . . 40

4.8 Estabilidade . . . . . . . . . . . . . . . . . . 40 
4.8.1 Estabilidade Robusta . . . . . . . . . . . . . . . . . . . . 41

4.9 Desempenho . . . . . . . . . . . . . . . . . . . . 42

4.9.1 Desempenho Robusto . . . . . . . . . . . . . . . . . . . 43

4.9 .2 Formatação das Funções de Sensibilidade . . . . . . . . . . . . . . . 45

4.9 .3 Configuração de Dois Portos . . . . . . . . . . . . . . . . . . . 47

4.10 Metodologia da Sensibilidade Mista . . . . . . . . . . . . . . . . . . 48

4.11 Controlador . . . . . . . . . . . . . . . . . . . 48

4.11.1 Formulação do Problema de Controle . . . . . . . . . . . . . . . . . 49

5 Resultados $\quad 52$

5.1 Introdução . . . . . . . . . . . . . . . . . . . . . . . 52

5.2 Apresentação do Problema . . . . . . . . . . . . . . . . . . . . . 52

5.3 Modelo Não-linear . . . . . . . . . . . . . . . . . . . . . 52

5.4 Modelo Linear . . . . . . . . . . . . . . . . . . . . . . 56

5.4 .1 Análise do Modelo Linear do VSA . . . . . . . . . . . . . . . . . 57

5.4 .2 Normalização . . . . . . . . . . . . . . . . . . . . . . . 60

5.5 Síntese do Controlador $\mathcal{H}_{\infty} \ldots \ldots \ldots \ldots$. . . . . . . . . . . 61

5.5.1 Especificações do projeto . . . . . . . . . . . . . . . 62

5.5.2 Funções de ponderação . . . . . . . . . . . . . . . . . . . . . 62

5.5.3 Funções de Sensibilidade . . . . . . . . . . . . . . . . . . . 66

5.5.4 Resposta Temporal . . . . . . . . . . . . . . . 71

5.5.5 Resposta Linear . . . . . . . . . . . . . . . . . . 71

5.5.6 Resposta Modelo Não Linear . . . . . . . . . . . . . . . . . . . . . . 73

5.6 Controlador de Dois Graus de Liberdade . . . . . . . . . . . . . . . . . 80

5.7 Guiagem . . . . . . . . . . . . . . . . . . . 83

6 Conclusões $\quad 91$

6.1 Sugestões para trabalhos futuros . . . . . . . . . . . . . . . . . . 92 
Apêndice A - Hidrodinâmica do VSA 98

A.1 Coeficientes Hidrodinâmicos do Veículo . . . . . . . . . . . . . . . . . . 98

A.2 Modelo Linear do VSA . . . . . . . . . . . . . . . . . . . . . 100

Apêndice B - Espaços Normados 101

B.1 Espaços $\mathcal{H}_{2}$ e $\mathcal{H}_{\infty} \ldots \ldots \ldots \ldots \ldots 10 \ldots \ldots$

B.2 Definições para Sistemas . . . . . . . . . . . . . . . . . . . . . 102

B.3 Transformação Fracional Linear LFT . . . . . . . . . . . . . . . . . . . 103

Apêndice C - Métodos Numéricos 104

C.1 Método de Euler . . . . . . . . . . . . . . . . . . . . . . . 104

C.2 Método de Euler modificado . . . . . . . . . . . . . . . . . . . . 104

C.3 Método Runge Kutta . . . . . . . . . . . . . . . . . . . 105

C.4 Simulação Computacional . . . . . . . . . . . . . . . . 105

Apêndice D - Código fonte $\quad 106$

D.1 Síntese do Controlador . . . . . . . . . . . . . . . . . . . . 111 


\section{Lista de Figuras}

2.1 a) Casco Carmichael b) Casco torpedo. . . . . . . . . . . . . . 7

2.2 Arranjo Geral de um VSA típico. . . . . . . . . . . . . . . . 8

2.3 Diagrama de blocos de navegação, guiagem e controle para o veículo submersível. . . . . . . . . . . . . . . . . . . . . . 8

2.4 Superfícies de controle do VSA . . . . . . . . . . . . . . . . . . . 10

3.1 Sistema de coordenadas para o VSA, referencial fixo e referencial móvel. . . 12

3.2 Translação e rotação do referencial móvel relativamente ao referencial fixo. 15

3.3 Resistência de avanço para o VSA. . . . . . . . . . . . . . 20

3.4 Curvas características do propulsor para o veículo submersível autônomo

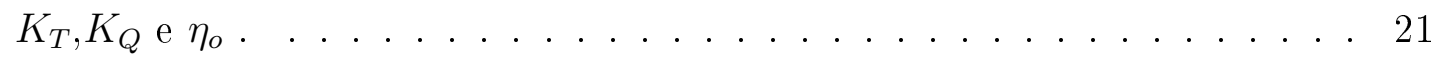

4.1 Controlador Clássico - SISO . . . . . . . . . . . . . . . . 31

4.2 Matriz Pequena e Grande, valores singulares. . . . . . . . . . . . . . . 35

4.3 Incerteza aditiva. . . . . . . . . . . . . . . . . . . 37

4.4 Incerteza multiplicativa na saída. . . . . . . . . . . . . . . . . 37

4.5 Barreiras de Robustez e Especificações para $S$ e T. . . . . . . . . . . . . 39

4.6 Estabilidade Robusta, SISO . . . . . . . . . . . . . . . . . . . . . 42

4.7 Desempenho Robusto . . . . . . . . . . . . . . . . . . . . . 44

4.8 Função de Ponderação $W_{s} \ldots \ldots \ldots$. . . . . . . . . . . . . 46

4.9 Função de Ponderação $W_{u} \ldots \ldots \ldots$. . . . . . . . . . . . . . 46

4.10 Configuração Genérica de Dois Portos. . . . . . . . . . . . . . . . . . 47

5.1 Modelo não-linear sem controle: $\delta_{r}=15^{\circ}$ e $\delta_{s}=0^{\circ} \ldots \ldots \ldots \ldots$

5.2 Modelo não-linear sem controle: $\delta_{r}=-15^{\circ}$ e $\delta_{s}=0^{\circ} \ldots \ldots \ldots$ 
5.3 Modelo não-linear sem controle: $\delta_{r}=0^{\circ}$ e $\delta_{s}=15^{\circ} \ldots \ldots \ldots \ldots \ldots$

5.4 Modelo não-linear sem controle: $\delta_{r}=0^{\circ}$ e $\delta_{s}=-15^{\circ} \ldots \ldots \ldots \ldots$

5.5 Modelo Linear Invariante no Tempo do Veículo Submersível Autônomo, . . 57

5.6 Distribuição de pólos e zeros do modelo linear do VSA. . . . . . . . . . . . 58

5.7 Magnitude dos autovetores associados a seus autovalores correspondentes $\lambda_{*} 59$

5.8 Valores Singulares de $G(s)$ em malha aberta. . . . . . . . . . . . 60

5.9 Configuração geral para síntese. . . . . . . . . . . . . . . . . . . 61

5.10 Matriz de transferência da função de ponderação $\left(1 / W_{S}\right) \ldots$. . . . . . . . 64

5.11 Matriz de transferência da função de ponderação $\left(1 / W_{T}\right) \ldots$. . . . . . . 65

5.12 Matriz de transferência da função de ponderação $\left(1 / W_{C}\right) \ldots$. . . . . . . . 65

5.13 Função de sensibilidade $(S)$ e inversa da função de ponderação $\left(1 / W_{S}\right)$ . 68

5.14 Função de sensibilidade complementar $(T)$ e inversa da função de ponderação $\left(1 / W_{T}\right) \ldots \ldots \ldots \ldots \ldots$

5.15 Função de sensibilidade do controlador $(C)$ e inversa da função de ponderação $\left(1 / W_{C}\right) \ldots \ldots \ldots \ldots$. . . . . . . . . . . . . . . . . 69

5.16 Matriz de Transferência $W_{S} S \ldots \ldots \ldots \ldots$

5.17 Matriz de Transferência $W_{T} T \ldots \ldots \ldots \ldots$

5.18 Matriz de Transferência $W_{C} C \ldots \ldots \ldots \ldots$. . . . . . . . . 71

5.19 Resposta ao degrau unitário do modelo linear controlado: balanço, caturro, velocidade de guinada e velocidade de submersão. . . . . . . . . . . . . . . 72

5.20 Resposta ao degrau unitário do modelo não linear controlado: balanço, caturro, velocidade de guinada e velocidade de submersão. . . . . . . . . . 74

5.21 Resposta ao degrau unitário para o caturro . . . . . . . . . . . . 76

5.22 Caso 1; velocidade e posição do veículo controlado (degrau unitário no caturro e velocidade de avanço de $u=2,7 \mathrm{~m} / \mathrm{s}) \ldots \ldots . . \ldots 77$

5.23 Caso 2; velocidade e posição do veículo controlado (degrau unitário no caturro e velocidade de avanço de $u=1,1 \mathrm{~m} / \mathrm{s}) \ldots \ldots . \ldots 78$ 
5.24 Resposta não linear do sistema controlado considerando incertezas de massa adicionada . . . . . . . . . . . . . . . . . . 79

5.25 Controlador de dois graus de liberdade, configuração genérica de dois portos. 81

5.26 Resposta ao degrau unitário com o controlador de 2-GL - caso não linear. . 82

5.27 Resposta ao degrau 0,5 com o controlador de 2-GL - caso não linear. . . . . 82

5.28 Diagrama de Blocos: Guiagem e Controle para o VSA. . . . . . . . . . . . 84

5.29 Trajetória desejada de acordo com LOS: Os alvos são representados por pequenos círculos, e o círculo maior representa a restrição imposta $\left(\right.$ raio $\left.=\rho_{o}\right) .84$

5.30 Trajetória circular: $u=2 \mathrm{~m} / \mathrm{s} \ldots \ldots \ldots \ldots \ldots$. . . . . . . . 85

5.31 Trajetória circular: movimento dos lemes. . . . . . . . . . . . . . 85

5.32 Trajetória Circular: sinal de comando. . . . . . . . . . . . . . . 86

5.33 Trajetória circular: resposta do sistema controlado. . . . . . . . . . . . 86

5.34 Trajetória Circular, posição $x, y$ e $z$ do VSA. . . . . . . . . . . . . . 87

5.35 Trajetória zig-zag: $u=2 \mathrm{~m} / \mathrm{s} . \ldots \ldots \ldots$. . . . . . . . 87

5.36 Trajetória zig-zag: movimento dos lemes. . . . . . . . . . . . . 88

5.37 Trajetória zig-zag: sinal de comando. . . . . . . . . . . . . . 88

5.38 Trajetória zig-zag: resposta do sistema controlado. . . . . . . . . . . . . . 89

5.39 Trajetória zig-zag: posição $x, y$ e $z$ do VSA. . . . . . . . . . . . . . 89

5.40 Trajetória helicoidal do VSA . . . . . . . . . . . . . . . . . 90

B.1 Transformações Fracionais Lineares . . . . . . . . . . . . . . . . . . . . 103 


\section{Lista de Tabelas}

2.1 Descrição do Veículo Submersível Autônomo . . . . . . . . . . . . . . . . . 5

3.1 Sistema de Coordenadas . . . . . . . . . . . . . . . . . . . . 11

5.1 Pólos do Sistema Linear Invariante no Tempo . . . . . . . . . . . . . . 57

5.2 Autovalores e Autovetores da Planta $G(s) \ldots \ldots \ldots$. . . . . . . 58

5.3 Resposta do sistema, assumindo um tempo de acomodação $T=4 \tau$. . . . 62

5.4 Funções de Sensibilidade do sistema controlado . . . . . . . . . . . . . . 66

A.1 Parâmetros hidrodinâmicos normalizados para o VSA em 6 DOF . . . . . . 98

A.2 Parâmetros hidrodinâmicos para o VSA em 6 DOF . . . . . . . . . . . . 99 


\section{Lista de Abreviaturas}

VSA Veículo Submersível Autônomo

GL Grau de Liberdade

LIT Linear Invariante no Tempo

EDOs Equações Diferenciais Ordinarias

CFD Dinâmica de Fluidos Computacionais (Computational Fluid Dynamics)

SISO Sistema dinâmico escalar, uma entrada e uma saída (Single Input and Single Output)

MIMO Sistema dinâmico multivariável, várias entradas e várias saídas (Multiple Input and Multiple Output)

ER Estabilidade Robusta

DR Desempenho Robusto 


\section{Lista de Símbolos}

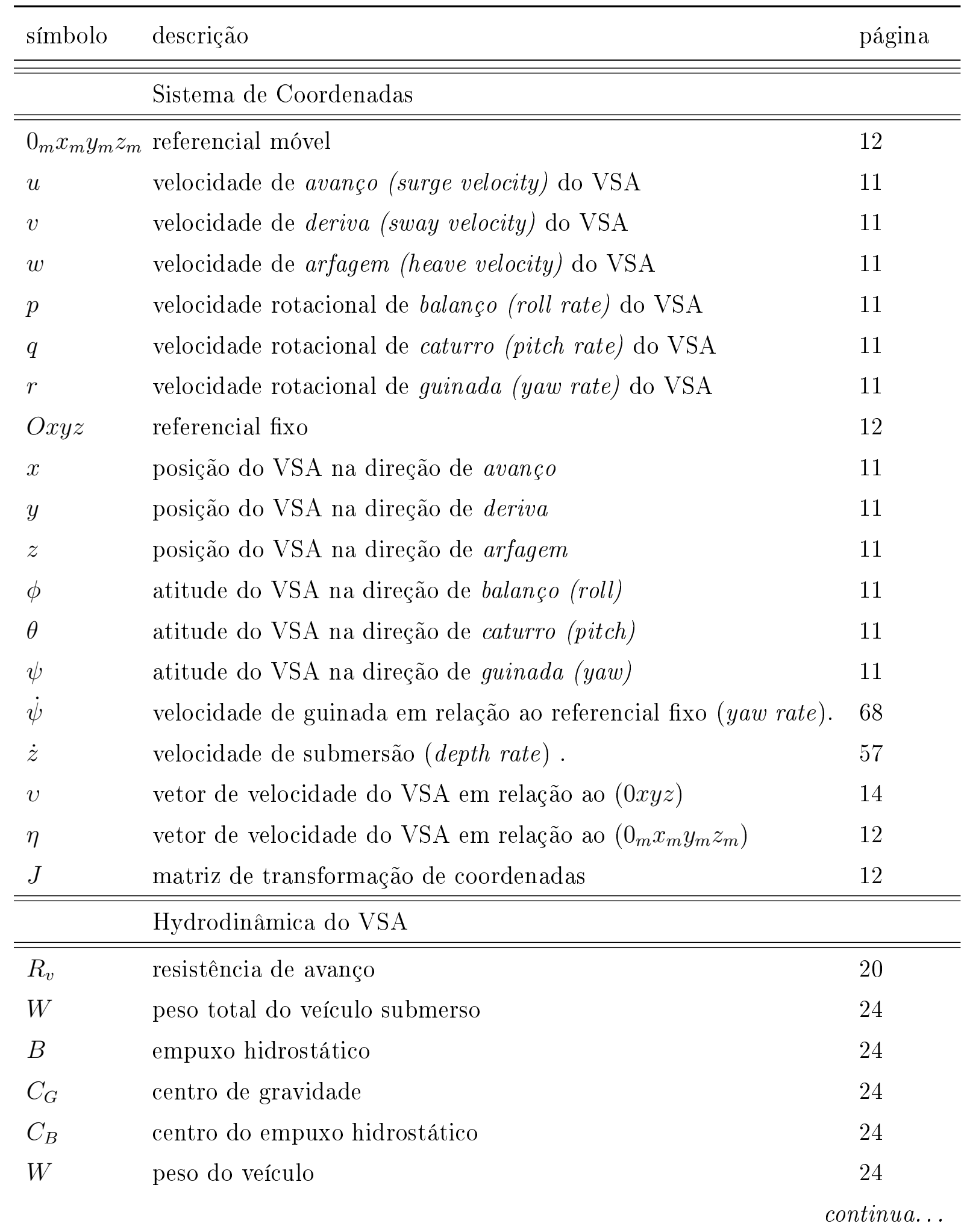




\begin{tabular}{|c|c|c|}
\hline símbolo & descrição & página \\
\hline$M_{A}$ & matriz de massa adicional & 18 \\
\hline$K_{T}$ & coeficiente de empuxo do propulsor & 21 \\
\hline \multirow{2}{*}{$K_{Q}$} & coeficiente de torque do propulsor & 21 \\
\hline & Controle Avançado & \\
\hline$x(t)$ & vetor de controle do sistema & 31 \\
\hline$y(t)$ & vetor de saída do sistema & 31 \\
\hline$u(t)$ & vetor de estados do sistema & 31 \\
\hline$A$ & matriz de estados do sistema & 31 \\
\hline$B$ & matriz de controle do sistema & 31 \\
\hline$C$ & matriz de observador de estados do sistema & 31 \\
\hline$D$ & matriz do sistema & 31 \\
\hline$G_{\triangle}$ & planta Real & 37 \\
\hline G & planta Nominal & 31 \\
\hline $\mathbb{R}$ & campo dos números reais & 31 \\
\hline $\mathcal{R}$ & matrizes de transferência próprias, racionais e reais & 101 \\
\hline$\triangle_{A}$ & incerteza Aditiva & 37 \\
\hline$\triangle_{M}$ & incerteza Multiplicativa & 37 \\
\hline$S$ & função de sensibilidade & 38 \\
\hline$T$ & função de sensibilidade complementar & 38 \\
\hline$C$ & $(K S)$ função de sensibilidade do controlador & 38 \\
\hline$W_{S}$ & função de ponderação da sensibilidade & 45 \\
\hline$W_{T}$ & função de ponderação da sensibilidade complementar & 45 \\
\hline$W_{C}$ & função de ponderação da sensibilidade do controlador & 45 \\
\hline$P(s)$ & Planta Generalizada & 48 \\
\hline$K(s)$ & controlador & 48 \\
\hline$w$ & distúrbios externos ou entradas exogenas & 61 \\
\hline$z$ & sinal do erro & 61 \\
\hline$L(s)$ & função de transferência de malha aberta & 42 \\
\hline
\end{tabular}




\section{Introdução}

Atividades submarinas cada vez mais intensas, principalmente no que se refere à exploração de recursos marinhos, pesquisas biológicas e aspectos relativos à segurança nacional dos países têm levado ao estudo e ao desenvolvimento de diversos tipos de veículos submersíveis de pequeno porte. Os mini-submersíveis mais comuns atualmente são classificados em: VSORs - veículos submersíveis operados de forma remota (ROV: Remotely Operated Vehicles) e VSAs - veículos submersíveis autônomos (AUV: Autonomous Underwater Vehicle). Uma classificação mais detalhada e extensiva pode ser encontrada em (SORANI, 2002).

O potencial tecnológico e econômico dos veículos submersíveis autônomos é apreciável, principalmente, em atividades como busca e prospecção de petróleo e gás natural em águas profundas; coleta de dados para pesquisas relacionadas ao meio-ambiente e à biologia marinha; inspeção de grandes estruturas flutuantes ou subaquáticas além da atuação em missões militares e de guerra para a segurança nacional.

Este trabalho é parte de uma linha de pesquisa dos Departamentos de Engenharia Mecânica e Engenharia Naval e Oceânica da Escola Politécnica da USP que visa à construção veículos submersíveis de pequeno porte. O veículo estudado neste trabalho deverá ser autônomo, operar a grandes profundidades, ser autopropelido, ser dotado de controle automático e ter capacidade de visão e manipulação no fundo do mar. O objetivo deste trabalho é projetar um controlador que possa garantir a manobrabilidade do veículo no meio offshore. Para atingir este propósito, desenvolveu-se um modelo físico-matemático de ordem completa (seis graus de liberdade) que foi simulado em computador. A partir da solução numérica do modelo foi possível observar o forte acoplamento das dinâmicas, e confirmou-se a natureza muito não linear do sistema.

As técnicas de controle para VSAs utilizam, geralmente, modelos aproximados ou modelos de ordem reduzida. As técnicas de controle mais utilizadas requerem, em geral, modelos lineares aproximados, e na maioria dos casos de ordem reduzida, obtidos desacoplandose as dinâmicas do veículo em subsistemas. Neste trabalho, ao contrário, utilizaram-se técnicas de controle robusto e sintetizou-se um controlador a partir do modelo linear apro- 
ximado de ordem completa. O sistema controlado foi simulado, tanto para o caso linear como para o sistema completo não linear.

\subsection{Motivação e Objetivos}

Uma ampla discussão sobre VSAs pode ser encontrada em Valavanis et al. (1997). As pesquisas sobre veículos submersíveis autônomos têm sido desenvolvidas desde a década de sessenta. Alguns protótipos foram desenvolvidos na década de oitenta. Os VSAs são capazes de operar em diversos ambientes, incluindo o litoral de águas rasas e sob as camadas de gelo polares. Eles podem detectar objetos utilizando sonares ou câmeras de vídeo, podem ainda medir temperatura, a salinidade da água, etc. Estas características são interessantes para pesquisas oceanográficas e operações militares como a localização de minas e reconhecimento de portos.

O desenvolvimento do estudo de VSAs tem levado à elaboração de modelos matemáticos complexos, incluindo modelos não-lineares com seis graus de liberdade (CANHETTI, 1998; PRESTERO, 2001). Em geral, estes modelos têm um alto acoplamento entre suas variáveis, o que dificulta sobremaneira o desenvolvimento de um controlador. Técnicas de controle clássico têm sido implementadas para sistemas desacoplados com poucos graus de liberdade, com resultados satisfatórios. Um projeto de controle envolvendo todos os graus de liberdade demanda, naturalmente, um esforço bem maior.

Existem poucos trabalhos, onde o controle de VSAs é feita de maneira centralizada e multivariável. O uso de modelos centralizados de ordem elevada permite que o VSA realize manobras muito mais complexas no meio 3D submarino.

O objetivo desta dissertação, em contraposição à abordagem clássica, é projetar um controlador centralizado multivariável para os seis graus de liberdade de um VSA, garantindo estabilidade e desempenho robustos, apesar de distúrbios, ruídos e incertezas de modelagem.

\subsection{Revisão Bibliográfica}

Existem inumeráveis estratégias de controle que podem ser implementadas em veículos submersíveis. Dada a natureza do problema, estratégias SISO (single Input and Single output) adquirem pouca relevância e estratégias MIMO (Multiple Input and Multiple Output) são as mais adequadas no projeto do controlador.

Este trabalho começa pelo estudo e validação do modelo matemático do VSA. McEwen 
(2002) e (SONG; AN; SMITH, 2002) disponibilizam modelos matemáticos de VSAs com todos seus coeficientes hidrodinâmicos. Estes veículos têm características similares ao VSA usado neste trabalho, o que permitiu realizar uma comparação e verificação do modelo e coeficientes hidrodinâmicos desenvolvidos por Canhetti (1998). Não foi feita uma revisão exaustiva do modelo, o que sem dúvida, conduziria à realização de testes em tanques de prova. Recomenda-se aqui o trabalho de Prestero (2001), onde a modelagem é abordada do ponto de vista experimental, com auxílio de tanques de prova e MMP (Mecanismos de Movimento Planar).

Para aprendizagem dos conceitos hidrodinâmicos, usou-se a apostila elaborada por Triantafyllou e Hover (2003) no curso Maneuvering and Control of Surface and Underwater Vehicles, MIT, onde abordam-se tópicos relevantes relativos ao estudo hidrodinâmico de veículos submersíveis como estabilidade, arrasto hidrodinâmico, sustentação, propulsão, teoria de corpos esbeltos, etc. Para melhor entendimento do assunto recomenda-se ainda os trabalhos de (ABKOWITZ, 1969; NEWMAN, 1977).

O modelo não linear obtido, foi linearizado de acordo com o muito bem conhecido método de perturbações, expostos em (MEIROVITCH, 1985). Porém, o modelo linear foi também analisado por abordagens de autovalores e autovetores, expostos em (KWAK, 1999), permitindo quantificar o acoplamento existente entre cada uma das dinâmicas do submersível. A característica multivariável dos modelos de VSAs, induzem e motivam fortemente o uso de técnicas de controle avançado, robusto e multivariável. Existe uma extensa variedade de estratégias de Controle Robusto, muitas difíceis de entender e de implementar. Entre as consultadas destacam-se: o Controle por Modos Deslizantes, o Controle Fuzzy, o Controle $L Q G / L T R$ e o Controle $\mathcal{H}_{2}$ e $\mathcal{H}_{\infty}$. O Controle Robusto garante a estabilidade e o desempenho do sistema, mesmo em condições onde perturbações, ruídos e incertezas de modelagem estejam presentes e por isso foi a abordagem escolhida.

Para o caso multivariável, aplicações de Controle por Modos Deslizantes em veículos submersíveis podem ser encontradas nos trabalhos de Healey e Lienard (1993), Fossen (1994) e Souza (2003). Esta técnica é muito complexa quando aborda sistemas multivariáveis (LOGAN, 1993). O Controle Fuzzy pode ser utilizado como uma estratégia quando carece-se do conhecimento do modelo do veículo, e através de regras simples resolve-se o problema de controle. O Controle Fuzzy pode também ser usado otimizando as respostas de um Controlador Robusto (SONG; AN; SMITH, 2002). O Controle Robusto LQG/LTR, somente aplica-se em sistemas quadrados (número de variáveis de controle igual ao número de variáveis controladas). Aplicações em veículos submersíveis encontram-se em Martin, Athans e Valvani (1986), Naeem, Sutton e M.Ahmad (2003).

O Controle Robusto $\mathcal{H}_{\infty}$ multivariável, técnica adotada neste trabalho, também foi uti- 
lizado para o controle de VSAs por outros autores, cabendo destacar os trabalhos de Whitcomb (2000), Silvestre e Pascoal (2005); porém nestes trabalhos, a dinâmica do submersível é dividida em susbsitemas de ordem reduzida.

Finalmente foi revisada uma extensa bibliografia relativa ao Controle Robusto $\mathcal{H}_{\infty}$ (ZHOU; DOYLE; GLOVER, 1995; SKOGESTAD; POSTLETHWAITE, 1996; LUNDSTRÖM; SKOGESTAD; DOYLE, 1999; ZHOU, 2000; TANNER; GEERING, 2003). Porém, a apostila elaborada por Donha (2003), para sistemas de controle multivariáveis, foi chave neste estudo. A técnica de Sensibilidade Mista $\mathcal{H}_{\infty}$ foi proposta para resolver o problema de controle. Também, é preciso salientar o trabalho de Donha e Katebi (2004) na otimização de parâmetros importantes para a síntese do controlador centralizado.

\subsection{Estrutura da Dissertação}

Para descrever os trabalhos realizados, optou-se por dividir a dissertação em seis capítulos, as referências bibliográficas e finalmente vários apêndices.

No capítulo 2 apresenta-se uma descrição do VSA utilizado neste trabalho.

No capítulo 3 apresentam-se os conceitos hidrodinâmicos e a formulação matemática do modelo VSA.

No capítulo 4 apresenta-se a estratégia de controle avançada para o VSA.

No capítulo 5 apresenta-se a implementação da estratégia de controle no VSA, resultados e testes do modelo (linear e não linear) controlado, visa-se atender os requisitos de controle.

No capitulo 6 apresentam-se as conclusões e comentários deste trabalho. A seguir são apresentados as Referências Bibliográficas e os Apêndices. 


\section{Descrição do Veículo}

Este capítulo trata da descrição física do Veículo Submersível Autônomo (VSA) escolhido para a elaboração desta dissertação. São apresentadas as dimensões principais, as características estruturais, a forma do casco, os sistemas de propulsão, de energia, de controle, sistemas de navegação e posicionamento e sistemas auxiliares.

\subsection{Características principais}

A forma hidrodinâmica do casco de um veículo é determinada por características de propulsão, estabilidade e manobrabilidade a diferentes velocidades. A forma do casco impõe limitações de acesso, lançamento e recuperação, manutenção e modularidade.

A tabela 2.1 abaixo apresenta as principais características físicas do VSA utilizado na consecução deste trabalho. As velocidades podem variar entre $1 \mathrm{e} 6 \mathrm{~m} / \mathrm{s}$, e a velocidade de cruzeiro é $2 \mathrm{~m} / \mathrm{s}$.

Tabela 2.1: Descrição do Veículo Submersível Autônomo

\begin{tabular}{lcrc}
\hline \hline Massa & $m$ & 1428,6 & $\mathrm{~kg}$ \\
Comprimento total & $l$ & 5,989 & $\mathrm{~m}$ \\
Diâmetro & $d$ & 0,624 & $\mathrm{~m}$ \\
Deslocamento submerso & $D_{s}$ & 14000 & $\mathrm{~N}$ \\
\hline
\end{tabular}

São ítens importantes na seleção do casco para o VSA:

- redução do arrasto total do veículo

- regime de escoamento laminar

- estabilidade e manobrabilidade adequada em velocidades baixas. 
O arrasto de fricção e o arrasto de forma determinam o arrasto total do veículo na modelagem adotada. O arrasto de fricção varia em função da velocidade e da área superficial exposta; assim, cascos pequenos com menor área superficial oferecem menor arrasto de fricção. O arrasto de forma é função da forma geométrica do veículo e do fenômeno de separação. Geralmente formas geométricas longas e esbeltas minimizam a separação e em conseqüência o arrastro de forma diminui.

Numa perspectiva simples, a forma de casco que promove escoamento laminar na camada limite é a melhor escolha. O casco Carmichael desenvolvido e testado por Bruce Carmichael nos anos setenta (ISE, 2000), é um bom exemplo deste tipo de casco. No escoamento laminar as partículas se movimentam em lâminas ou camadas. $\mathrm{O}$ arrasto de fricção aumenta no escoamento turbulento, onde as partículas de fluido se movimentam desordenadamente, gerando altas pressões sobre a superfície do casco.

Para garantir o escoamento laminar, o casco deve ser projetado de maneira tal que a seção transversal se incremente gradualmente a partir da proa, promovendo uma gradiente de pressão favorável, na ordem de 60-70\% do casco. Nesta região, a superfície deve ser lisa e hidrodinamicamente o mais limpa possível. Superfícies de controle (hidroplanos) não podem ser instaladas próximos à proa, pois podem desestabilizar o escoamento laminar. Em conseqüência, elas aparecem na parte posterior do casco. Sensores acústicos, antenas de comunicação e demais sensores devem também ser instaladas o mais distante possível da proa.

A ilustração da figura 2.1 mostra duas formas alternativas de casco: a primeira produz um escoamento perfeitamente laminar, já a forma de torpedo produz um escoamento semilaminar. Porém, o casco torpedo tem sido a escolha mais freqüente dos projetistas há um século atrás, devido à modularidade, fácil construção e também a maior disponibilidade de espaço para a instalação do equipamento eletrônico. A forma geométrica do torpedo também facilita a tarefa de modelagem e a obtenção de parâmetros hidrodinâmicos.

Para veículos da forma torpedo, Prestero (2001) em testes experimentais, baseado nos estudos de Hoerner $(1965,1992)$, afirma que as formas hidrodinâmicas com uma taxa comprimento $(l)$ e diâmetro $(d)$ entre 6 e 11 são ótimas para minimizar o arrasto total do veículo. Assim, o casco torpedo produz um escoamento semi-laminar e semi-turbulento a velocidades baixas $(2 \mathrm{~m} / \mathrm{s})$.

A forma de casco torpedo (ver figura 2.1) é a forma do veículo submersível estudado neste trabalho, tem um nariz parabólico, seguido de uma seção cilíndrica no meio, e na popa estão localizadas as superfícies de controle e o propulsor, as dimensões exatas e planos de construção podem-se encontrar em (CANHETTI, 1998). 


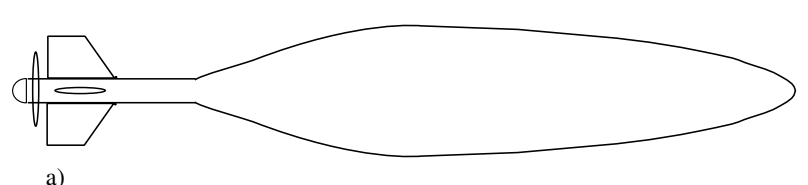

a)

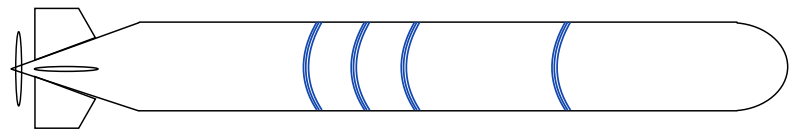

b)

Figura 2.1: a) Casco Carmichael b) Casco torpedo.

\section{$2.2 \quad$ Arranjo geral}

O arranjo geral do veículo está dividido em 6 partes

1. Popa: propulsor, lemes, eixo e hidrofones

2. Motor: motor e motoredutores

3. Inversor: inversor, amplificadores e tanque de lastro de ré

4. Baterias: bateria de propulsão e baterias auxiliares

5. Computador: computador, sensor inercial e eletrônicos

6. Tanque de lastro: tanque de lastro de vante

7. Proa: lastro sólido e hidrofones

Os veículos submersíveis autônomos têm aplicações diversas, em conseqüência o número e tipo de sensores utilizados podem ser muito diferentes.

Primeiramente, destacam-se sensores acústicos que atendem os requisitos das aplicações marinhas, porém, há uma ampla gama de sensores não acústicos para medidas, como por exemplo medidores da qualidade da água para instalação de cabos submarinos.

Os sensores acústicos são utilizados em batimetria, desvio de obstáculo, cálculo da velocidade de avanço através do principio dopler. 


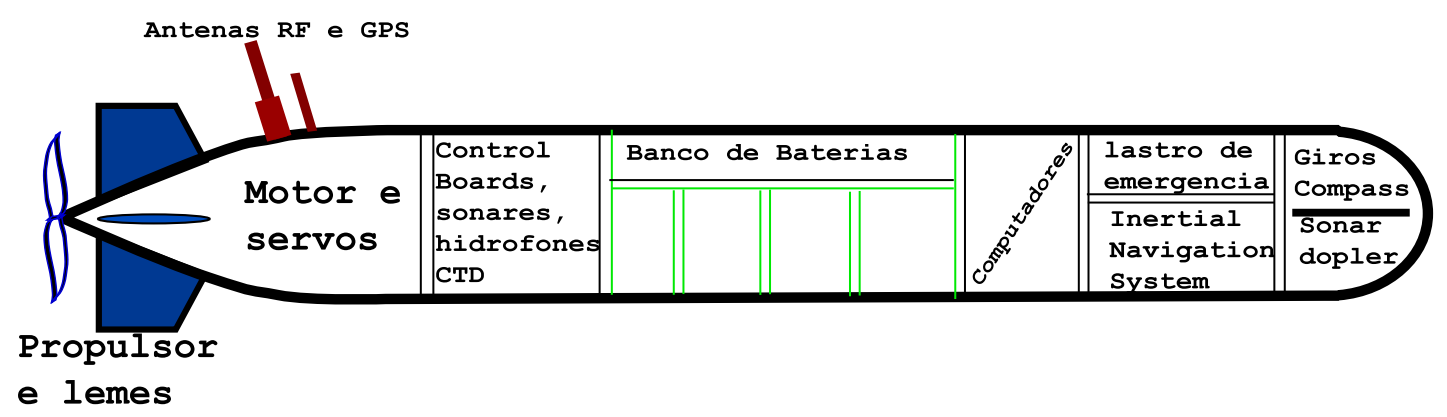

Figura 2.2: Arranjo Geral de um VSA típico.

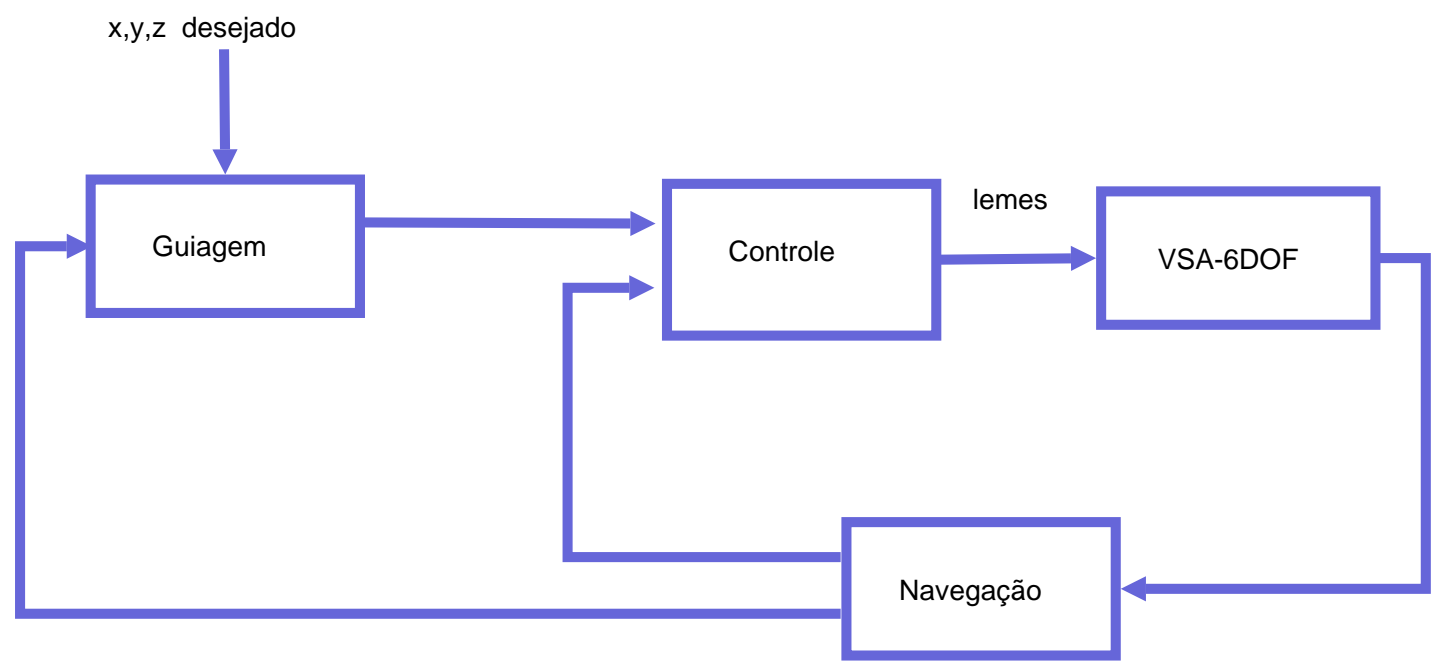

Figura 2.3: Diagrama de blocos de navegação, guiagem e controle para o veículo submersível.

\subsection{Sensores de Navegação}

Os sensores de navegação são equipamentos que permitem estimar a posição e atitude do veículo relativamente a um sistema de coordenadas $^{1}$, levando informações necessárias para os blocos de controle e guiagem (ver figura 2.3). O bloco de guiagem processa os dados, do sistema de navegação e da trajetória desejada e configura os sinais de comando para o bloco de controle. O bloco de controle aciona os atuadores a partir dos sinais observados pelo sistema.

Para o desenvolvimento do controlador multivariável desenvolvido nesta pesquisa, são necessárias quatro variáveis (ângulo de balanço, ângulo de caturro, velocidade de guinada e velocidade de submersão). Geralmente, para um VSA desenvolve-se um controlador independente para o avanço; assim, é instalado um sensor de velocidade de avanço. Para a guiagem são necessárias três variáveis de posição $x, y$ e $z$. Estas podem ser obtidas por

\footnotetext{
${ }^{1}$ Os sistemas de coordenadas serão expostos no capítulo seguinte
} 
um algoritmo de estimação.

Os giroscópios provêem três medidas angulares, e com ajuda de algoritmos de estimação, como os filtros de Kalman, podem-se conseguir as variáveis de estado inacessíveis do sistema.

A precisão dos sensores de navegação está fortemente associada ao custo da tecnologia usada, e missões de longa duração precisam de instrumentos de melhor qualidade (ISE, 2000).

\subsection{Lastro}

Nos projetos de veículos submersíveis é necessário projetar sistemas de lastro para compensar o empuxo hidrostático, conseguindo assim submergir e emergir o veículo. São utilizados tanques de lastro equipados com bombas hidráulicas e sistemas de ar comprimido. É preciso ressaltar que a utilização destes sistemas é limitada pela profundidade da missão. Para o VSA, inicialmente os tanques de lastro estão cheios de água, garantindo o equilibro hidrostático neutro, de forma que caso o sistema de propulsão falhe, seja acionado o sistema de ar comprimido, expulsando toda a água dos tanques de lastro e assim o veículo possa voltar à superfície.

\subsection{Energia}

A potência instalada é função da resistência de avanço do casco hidrodinâmico na velocidade máxima desejada. Domiciniano (1996) propõe critérios para alcançar o regime de escoamento turbulento, o que resultou na adoção das seguintes características:

- Potência instalada $=11,0 \mathrm{KW}$;

- Velocidade Máxima $=6 \mathrm{~m} / \mathrm{s}$ (11 nós);

- Autonomia a $65 \%$ de potência $=1$ hora.

\subsection{Superfícies de Controle}

Movimentos de rotação ou submersão de submersíveis podem ser efetuados pela ação de superfícies de controle. O veículo escolhido conta com duas superfícies de controle 

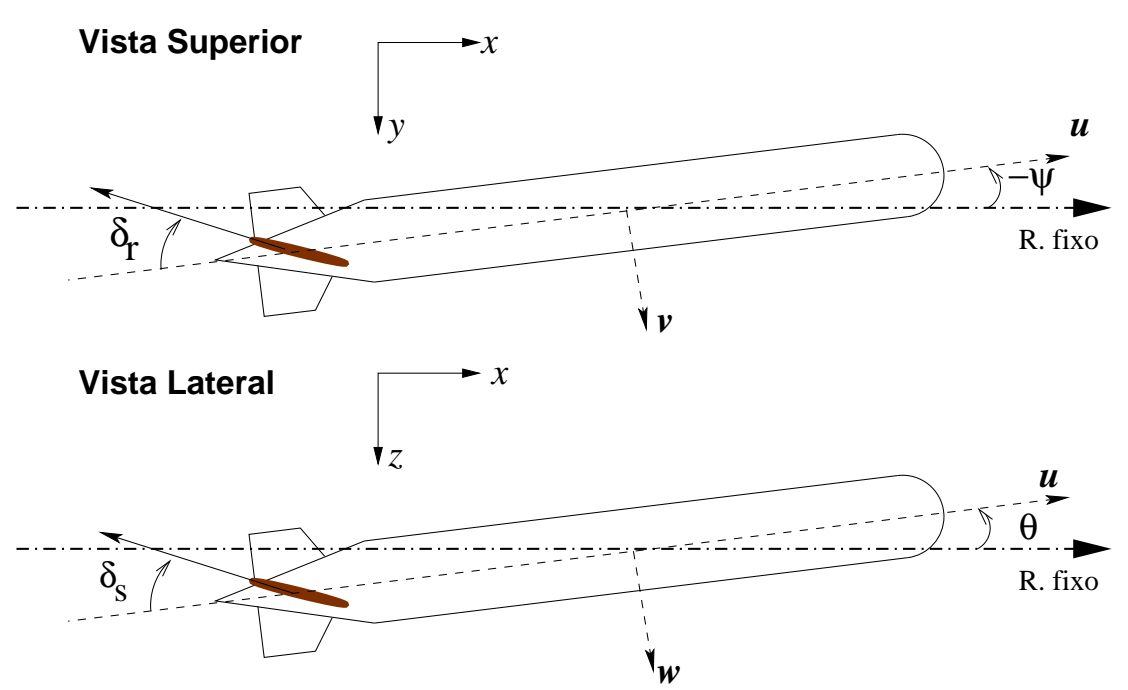

Figura 2.4: Superfícies de controle do VSA.

para realizar estes movimentos: leme horizontal e leme vertical, os quais permitem dirigir o veículo numa determinada direção programada pelo subsistema de guiagem. O leme vertical faz com que o veículo altere seu ângulo de rumo $(\psi)$. O leme horizontal altera o ângulo de caturro $(\theta)$, conforme ilustra a figura 2.4 .

Os valores máximos permitidos para a inclinação destes lemes no veículo escolhido são:

$\delta_{r}=30^{\circ}$ ângulo máximo permitido para o leme vertical.

$\delta_{s}=25^{\circ}$ ângulo máximo permitido para o leme horizontal. 


\section{Modelagem Dinâmica do Veículo}

\subsection{Introdução}

Neste capítulo definem-se as equações que governam o movimento do Veículo Submersível Autônomo (VSA). O modelo matemático consiste de equações cinemáticas e dinâmicas, sendo que as últimas incluem a descrição de forças e momentos externos.

\subsection{Sistema de Coordenadas}

Seis coordenadas são necessárias para determinar a posição e atitude de um veículo submersível no meio marinho. As três primeiras coordenadas descrevem o movimento linear do veículo ao longo do eixo $x, y$ e $z$, e as três últimas coordenadas descrevem o movimento rotacional do veículo. Os seis graus de liberdade do Veículo Submersível Autônomo são definidos como: avanço, deriva, arfagem, balanço, caturro e guinada; associados, respectivamente às variáveis $x, y$ e $z$ e rotações ao redor destes mesmos eixos, conforme apresenta a tabela 3.1.

Tabela 3.1: Sistema de Coordenadas

\begin{tabular}{r|cccc}
\hline & descrição & $\begin{array}{c}\text { forças e } \\
\text { momentos }\end{array}$ & $\begin{array}{c}\text { velocidade } \\
\text { linear e angular }\end{array}$ & $\begin{array}{c}\text { posição e } \\
\text { atitude }\end{array}$ \\
\hline \hline avanço & mov. na direção $x$ & $X$ & $u$ & $x$ \\
deriva & mov. na direção $y$ & $Y$ & $v$ & $y$ \\
arfagem & mov. na direção $z$ & $Z$ & $w$ & $z$ \\
balanço & rot. ao redor do eixo $x$ & $K$ & $p$ & $\phi$ \\
caturro & rot. ao redor do eixo $y$ & $M$ & $q$ & $\theta$ \\
guinada & rot. ao redor do eixo $z$ & $N$ & $r$ & $\psi$ \\
\hline
\end{tabular}




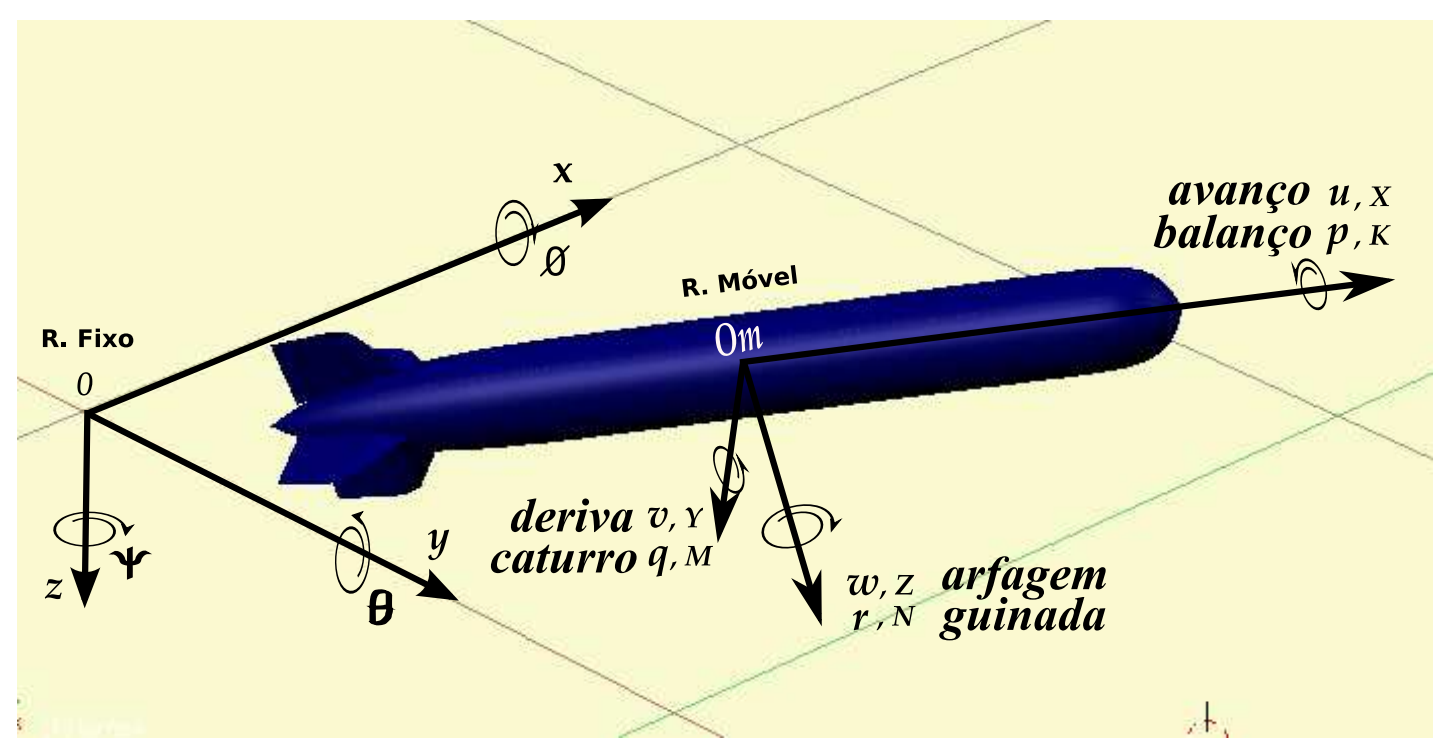

Figura 3.1: Sistema de coordenadas para o VSA, referencial fixo e referencial móvel.

\subsection{Cinemática do Veículo}

O movimento do sistema de referência móvel $0_{m} x_{m} y_{m} z_{m}$ (referencial móvel) é definido em relação a um sistema de referência inercial $0 x y z$ (referencial fixo), ver figura 3.1. O movimento do veículo em seis graus de liberdade pode ser descrito pelos seguintes vetores:

$$
\begin{gathered}
\eta_{1}=\left[\begin{array}{lll}
x & y & z
\end{array}\right]^{T} ; \quad \eta_{2}=\left[\begin{array}{lll}
\phi & \theta & \psi
\end{array}\right]^{T} ; \quad \eta=\left[\begin{array}{ll}
\eta_{1}, & \eta_{2}
\end{array}\right]^{T} \\
v_{1}=\left[\begin{array}{lll}
u & v & w
\end{array}\right]^{T} ; \quad v_{2}=\left[\begin{array}{lll}
p & q & r
\end{array}\right]^{T} ; \quad v=\left[\begin{array}{lll}
v_{1}, & v_{2}
\end{array}\right]^{T} \\
\tau_{1}=\left[\begin{array}{lll}
X & Y & Z
\end{array}\right]^{T} ; \quad \tau_{2}=\left[\begin{array}{lll}
K & M & N
\end{array}\right]^{T} ; \quad \tau=\left[\begin{array}{ll}
\tau_{1}, & \tau_{2}
\end{array}\right]^{T}
\end{gathered}
$$

onde $\eta$ descreve a posição e orientação do veículo em relação ao referencial fixo, $v$ a velocidade de translação e rotação em relação ao referencial móvel, e $\tau$ descreve as forcas e momentos totais que agem no veículo.

A figura 3.1 ilustra os sistemas de coordenadas do veículo submersível. A seguinte transformação de coordenadas relaciona as velocidades de translação entre o sistema de referência inercial e o sistema de referência móvel (FOSSEN, 1994):

$$
\left[\begin{array}{c}
\dot{x} \\
\dot{y} \\
\dot{z}
\end{array}\right]=J_{1}\left(\eta_{2}\right)\left[\begin{array}{lll}
u & v & w
\end{array}\right]^{T}
$$


onde:

$$
J_{1}\left(\eta_{2}\right)=\left[\begin{array}{ccc}
\cos \psi \cos \theta & \cos \psi \sin \theta \sin \psi-\sin \psi \cos \phi & \sin \psi \sin \phi-\cos \psi \cos \phi \sin \theta \\
\sin \psi \cos \theta & \cos \phi \cos \psi+\sin \phi \sin \theta \sin \psi & \cos \phi \sin \theta \sin \psi-\cos \psi \sin \phi \\
-\sin \theta & \cos \theta \sin \phi & \cos \phi \cos \theta
\end{array}\right]
$$

Note-se que $J_{1}\left(\eta_{2}\right)$ é ortogonal, quer dizer:

$$
\left[J_{1}\left(\eta_{2}\right)\right]^{-1}=\left[J_{1}\left(\eta_{2}\right)\right]^{T}
$$

A segunda transformação de coordenadas relaciona as velocidades rotacionais dos sistemas de coordenadas inercial e móvel:

$$
\left[\begin{array}{c}
\dot{\phi} \\
\dot{\theta} \\
\dot{\psi}
\end{array}\right]=J_{2}\left(\eta_{2}\right)\left[\begin{array}{lll}
p & q & r
\end{array}\right]^{T}
$$

onde:

$$
J_{2}\left(\eta_{2}\right)=\left[\begin{array}{ccc}
1 & \sin \phi \frac{\sin \theta}{\cos \theta} & \cos \phi \frac{\sin \theta}{\cos \theta} \\
0 & \cos \phi & -\sin \phi \\
0 & \frac{\sin \phi}{\cos \theta} & \frac{\cos \phi}{\cos \theta}
\end{array}\right]
$$

As matrizes $J_{1}$ e $J_{2}$ são matrizes de transformação de coordenadas. Note-se que $J_{2}$ não é definida para $\theta=90^{\circ}$. Isto não é problema sempre e quando o movimento do veículo não passe por esta singularidade, porém pode-se limitar $\theta$ nos valores próximos $\pm 89,99^{\circ} \mathrm{e}$ $\pm 90,01^{\circ}$. Outra alternativa é utilizar outra representação cinemática, como quaternions ou parâmetros de Rodriguez, ver (HUGHES, 1986).

\subsection{Dinâmica de Corpo Rígido do Veículo}

\subsubsection{Movimentos de Translação}

Inicialmente, descreve-se o movimento do veículo como um corpo rígido no sistema de coordenadas inercial. A lei fundamental de Newton estabelece que:

$$
\vec{F}=\frac{d}{d t}(m \vec{v})
$$

Onde $\vec{F}$ é a força total atuante no corpo rígido de massa $m$, é $\vec{v}$ é a velocidade com que o veículo se movimenta. O corpo rígido consiste de um extenso número de pequenas 
partículas, que podem ser indexadas, de forma que:

$$
\vec{F}=\sum_{i=1}^{N}\left(\vec{F}_{i}+\vec{R}_{i}\right)=\sum_{i=1}^{N} \frac{d}{d t}\left(m_{i} \vec{v}_{i}\right)
$$

onde $\vec{F}_{i}$ é a força externa atuando sobre a partícula e $\vec{R}_{i}$ é a força de reação exercida pelas demais partículas a seu ao redor (forças internas). Dado que o conjunto de partículas não é tratado de forma separada mediante forças internas, assume-se que se tem forças internas iguais é com sentidos opostos, tal que:

$$
\sum_{i=1}^{N} \vec{R}_{i}=\overrightarrow{0}
$$

A equação (3.8) se reduz a:

$$
\sum_{i=1}^{N} \vec{F}_{i}=\sum_{i=1}^{N} \frac{d}{d t}\left(m_{i} \vec{v}_{i}\right)
$$

Observa-se que as velocidades das partículas não são independentes, porque as partículas estão unidas de maneira rígida. Considerando-se, então, o sistema móvel, com a origem em $O_{m}$ e a partícula $i$ localizado a um raio $\vec{r}_{i}$ do referencial móvel, a expressão (3.10) escreve-se:

$$
\begin{aligned}
\sum_{i=1}^{N} \vec{F}_{i} & =\sum_{i=1}^{N} \frac{d}{d t}\left(m_{i}\left(\vec{v}_{o}+\vec{\omega} \times \vec{r}_{i}\right)\right) \\
& =m \frac{\partial \vec{v}_{o}}{\partial t}+\frac{d}{d t}\left[\vec{\omega} \times \sum_{i=1}^{N} m_{i} \vec{r}_{i}\right]
\end{aligned}
$$

onde $m=\sum_{i=1}^{N} m_{i}$ e $\vec{v}_{i}=\vec{v}_{o}+\vec{\omega} \times \vec{r}_{i}$. A seguir, define-se o vetor $\vec{r}_{G}$ como o vetor posição do centro de gravidade, e assim:

$$
m \vec{r}_{G}=\sum_{i=1}^{N} m_{i} \vec{r}_{i}
$$

o que conduz a equação (3.11) a:

$$
\sum_{i=1}^{N} \vec{F}_{i}=m \frac{\partial \vec{v}_{o}}{\partial t}+m \frac{d}{d t}\left(\vec{\omega} \times \vec{r}_{G}\right)
$$

Usando a expansão para a derivada total, a equação vetorial no sistema de coordenadas móvel é:

$$
\sum_{i=1}^{N} \vec{F}_{i}=m\left(\frac{\partial \vec{v}_{o}}{\partial t}+\vec{\omega} \times \vec{v}_{o}+\frac{d \omega}{d t} \times \vec{r}_{G}+\vec{\omega} \times\left(\vec{\omega} \times \vec{r}_{G}\right)\right)
$$




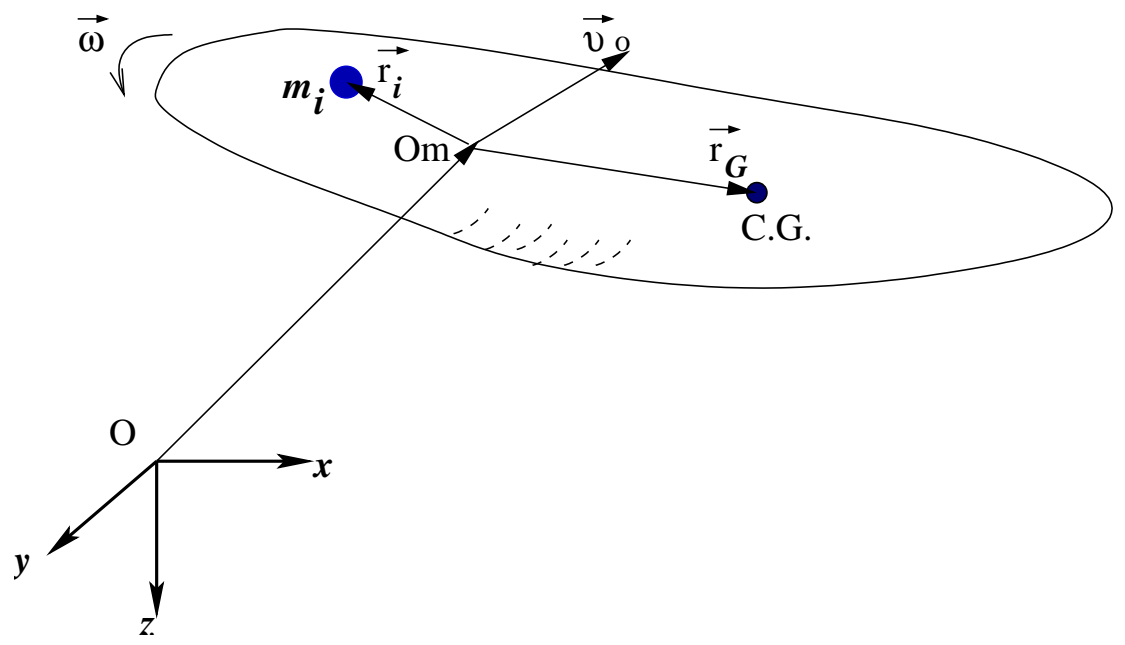

Figura 3.2: Translação e rotação do referencial móvel relativamente ao referencial fixo.

onde,

$\vec{v}_{o}=[u, v, w]^{T}$ é o vetor velocidade linear em relação ao referencial móvel;

$\vec{\omega}=[p, q, r]^{T}$ é o vetor velocidade angular em relação ao referencial móvel;

$\vec{r}_{G}=\left[x_{G}, y_{G}, z_{G}\right]^{T}$ é o vetor posição do centro de gravidade e

$\vec{F}=\left[X_{e x t}, Y_{e x t}, Z_{e x t}\right]^{T}$ é o vetor forças externas em relação ao referencial móvel.

Usando a identidade do triplo produto vetorial:

$$
\vec{\omega} \times\left(\vec{\omega} \times \vec{r}_{G}\right)=\left(\vec{\omega} \cdot \vec{r}_{G}\right) \vec{\omega}-(\vec{\omega} \cdot \vec{\omega}) \vec{r}_{G}
$$

a equação vetorial (3.14) se reduz à forma seguinte (TRIANTAFYLLOU; HOVER, 2003):

$$
\begin{aligned}
X_{\text {ext }} & =m\left[\dot{u}-v r+w q-x_{G}\left(q^{2}+r^{2}\right)+y_{G}(p q-\dot{r})+z_{G}(p r+\dot{q})\right] \\
Y_{\text {ext }} & =m\left[\dot{v}-w p+u r-y_{G}\left(r^{2}+p^{2}\right)+z_{G}(q r-\dot{p})-x_{G}(q p+\dot{r})\right] \\
Z_{\text {ext }} & =m\left[\dot{w}-u q+v p-z_{G}\left(p^{2}+q^{2}\right)+x_{G}(r p-\dot{q})+y_{G}(r q+\dot{p})\right]
\end{aligned}
$$

Note-se que nestas equações o centro de gravidade do veículo não coincide com o sistema de coordenadas móveis, quer dizer $\vec{r}_{G} \neq \overrightarrow{0}$.

\subsubsection{Movimentos de Rotação}

O momento angular de todas as partículas de um corpo rígido é dada pela equação:

$$
\sum_{i=1}^{N} \vec{M}_{i}=\sum_{i=1}^{N}\left(\vec{r}_{i} \times \vec{F}_{i}\right)=\sum_{i=1}^{N} \vec{r}_{i} \times \frac{d}{d t}\left(m_{i} \times \vec{v}_{i}\right),
$$


onde $\vec{M}_{i}$ é o momento externo aplicado sobre a partícula $i$. Procedendo-se da mesma maneira como para o movimento de translação, e usando a restrição de corpo rígido para as partículas tem-se:

$$
\begin{aligned}
\sum_{i=1}^{N} \vec{M}_{i}= & \sum_{i=1}^{N} m_{i} \vec{r}_{i} \times\left[\frac{\partial \vec{v}_{o}}{\partial t}+\vec{\omega} \times \vec{v}_{o}\right]+ \\
& \sum_{i=1}^{N} m_{i} \vec{r}_{i} \times\left(\frac{\partial \vec{\omega}}{\partial t} \times \vec{r}_{i}\right)+ \\
& \sum_{i=1}^{N} m_{i} \vec{r}_{i} \times\left(\vec{\omega} \times\left(\vec{\omega} \times \vec{r}_{i}\right)\right)
\end{aligned}
$$

Usando a relação (3.12) para o primeiro termo do lado direito da equação (3.18), ele se reduz a:

$$
m \vec{r}_{G} \times\left[\frac{\partial \vec{v}_{o}}{\partial t}+\vec{\omega} \times \vec{v}_{o}\right]
$$

O segundo termo pode ser expandido (usando o triplo produto interno) como:

$$
\begin{aligned}
\sum_{i=1}^{N} m_{i} \vec{r}_{i} \times\left(\frac{\partial \vec{\omega}}{\partial t} \times \vec{r}_{i}\right) & =\sum_{i=1}^{N} m_{i}\left(\left(\vec{r}_{i} \times \vec{r}_{i}\right) \frac{\partial \vec{\omega}}{\partial t}-\left(\frac{\partial \vec{\omega}}{\partial t} \cdot \vec{r}_{i}\right) \vec{r}_{i}\right) \\
& =\left\{\begin{array}{c}
\sum_{i=1}^{N} m_{i}\left(\left(y_{i}^{2}+z_{i}^{2}\right) \dot{p}-\left(y_{i} \dot{q}+z_{i} \dot{r}\right) x_{i}\right) \\
\sum_{i=1}^{N} m_{i}\left(\left(x_{i}^{2}+z_{i}^{2}\right) \dot{q}-\left(x_{i} \dot{q}+z_{i} \dot{r}\right) y_{i}\right) . \\
\sum_{i=1}^{N} m_{i}\left(\left(x_{i}^{2}+y_{i}^{2}\right) \dot{r}-\left(x_{i} \dot{q}+y_{i} \dot{r}\right) z_{i}\right)
\end{array}\right\}
\end{aligned}
$$

onde observa-se o aparecimento das definições de momentos e produtos de inércia como se segue:

$$
\begin{gathered}
I_{x}=\sum_{i=1}^{N} m_{i}\left(y_{i}^{2}+z_{i}^{2}\right) \\
I_{y}=\sum_{i=1}^{N} m_{i}\left(x_{i}^{2}+z_{i}^{2}\right) \\
I_{x}=\sum_{i=1}^{N} m_{i}\left(x_{i}^{2}+y_{i}^{2}\right) \\
I_{x y}=I_{y x}=-\sum_{i=1}^{N} m_{i} x_{i} y_{i} \\
I_{x z}=I_{z x}=-\sum_{i=1}^{N} m_{i} x_{i} z_{i} \\
I_{y z}=I_{z y}=-\sum_{i=1}^{N} m_{i} y_{i} z_{i}
\end{gathered}
$$


O terceiro termo da equação (3.18) pode ser trabalhado seguindo a mesma linha do segundo termo, chegando-se a:

$$
\begin{aligned}
\sum_{i=1}^{N} m_{i} \vec{r}_{i} \times\left(\vec{\omega} \times\left(\vec{\omega} \times \vec{r}_{i}\right)\right)= & \sum_{i=1}^{N} m_{i} \times \vec{r}_{i}\left(\left(\vec{\omega} \cdot \vec{r}_{i}\right) \vec{\omega}-(\vec{\omega} \cdot \vec{\omega}) \vec{r}_{i}\right) \\
= & \left\{\begin{array}{l}
\sum_{i=1}^{N} m_{i}\left(y_{i} r-z_{i} q\right)\left(x_{i} p+y_{i} q+z_{i} r\right) \\
\sum_{i=1}^{N} m_{i}\left(z_{i} p-x_{i} q\right)\left(x_{i} p+y_{i} q+z_{i} r\right) \\
\sum_{i=1}^{N} m_{i}\left(x_{i} q-y_{i} q\right)\left(x_{i} p+y_{i} q+z_{i} r\right)
\end{array}\right\} \\
= & \left\{\begin{array}{l}
I_{y z}\left(r^{2}-q^{2}\right)-I_{x z} p q-I_{x y} p r \\
I_{x z}\left(p^{2}-r^{2}\right)-I_{x y} r q-I_{y z} p q \\
I_{x y}\left(q^{2}-p^{2}\right)-I_{y z} p r-I_{x z} q r
\end{array}\right\}+ \\
& \left\{\begin{array}{l}
\left(I_{z}-I_{y}\right) r q \\
\left(I_{x}-I_{z}\right) r p \\
\left(I_{y}-I_{z}\right) p q
\end{array}\right\}
\end{aligned}
$$

Seja $\vec{M}=\left[K_{\text {ext }}, M_{\text {ext }}, N_{\text {ext }}\right]^{T}$ o momento externo total atuando sobre o veículo, i.e., o lado esquerdo da equação (3.18), a equação para o veículo se reduz à forma seguinte:

$$
\begin{aligned}
K_{e x t}= & I_{x} \dot{p}-I_{x y} \dot{q}-I_{x z} \dot{r}+\left(I_{z}-I_{y}\right) r q+I_{y z}\left(r^{2}-q^{2}\right)-I_{x z} p q+I_{x y} p r+ \\
& m\left[y_{G}(\dot{w}+p v-q u)-z_{G}(\dot{v}+r u-p w)\right] \\
M_{e x t}= & -I_{x y} \dot{p}+I_{y} \dot{q}-I_{y z} \dot{r}+\left(I_{x}-I_{z}\right) r p+I_{x z}\left(p^{2}-r^{2}\right)-I_{x y} r q+I_{y z} p q+ \\
& m\left[z_{G}(\dot{u}+q w-r v)-x_{G}(\dot{w}+p v-q u)\right] \\
N_{e x t}= & -I_{x z} \dot{p}-I_{y z} \dot{q}+I_{z} \dot{r}+\left(I_{y}-I_{x}\right) q p+I_{x y}\left(q^{2}-p^{2}\right)-I_{y z} p r+I_{x z} q r+ \\
& m\left[x_{G}(\dot{v}+r u-p w)-y_{G}(\dot{u}+q w-r v)\right]
\end{aligned}
$$

As equações de movimento do veículo em seis graus de liberdade, três de translação e três de rotação, são expressas de forma geral pelas equações (3.16) e (3.23).

\subsection{Coeficientes Hidrodinâmicos}

As equações de movimento do veículo incluem derivadas hidrodinâmicas, tais como $N_{v}$, $Z_{q}, K_{v}, Y_{\delta}$, etc. que dependem da forma do veículo, tamanho, distribuição de inércia e condições de equilíbrio. O tema de coeficientes hidrodinâmicos abrange uma grande área da mecânica de fluidos. Para sua determinação são usados métodos analíticos, numéricos e empíricos. Métodos analíticos provêm resultados para geometrias não muito complexas que podem ser usadas para comparar soluções obtidas por métodos numéricos. 
Devido à complexidade associada com o escoamento no meio marinho, dá-se preferência aos métodos empíricos no estudo da hidrodinâmica de estruturas marinhas. Isto principalmente porquê, em primeiro lugar, alguns problemas nessa área ainda esperam por uma solução numérica; segundo, porquê os métodos numéricos têm levado a resultados pouco satisfatórios e sua precisão precisa ser validada pelos outros métodos. É portanto aconselhável conduzir o projeto usando-se os três métodos. Trabalhos elaborados nesta ampla área podem ser encontrados em (HOVER, 1995; AVILA, 2003; BARROS; PASCOAL; SA, 2004; RENTSCHLER; HOVER; CHRYSSOSTOMIDIS, 2006). Neste trabalho apenas se adotará valores dos coeficientes hidrodinâmicos obtidos por Canhetti (1998), com algumas modificações para corrigir o comportamento do veículo submersível em seus seis graus de liberdade.

\subsubsection{Massa Adicionada}

Massas adicionadas podem ser entendidas como forças e momentos de pressão induzidas devido a um movimento harmônico forçado do corpo rígido e são proporcionais à aceleração do corpo (FOSSEN, 1994). Para corpos esbeltos pode ser aplicada a teoria de faixas para o cálculo destes coeficientes, integrando-se os coeficientes bi-dimensionais de massa adicionada ao longo do comprimento do veículo (NEWMAN, 1977).

Outra tentativa é usar ferramentas computacionais da dinâmica de fluidos (CFD), que resolvem o escoamento potencial ao redor do corpo rígido (BO, 2004). A matriz de massa adicionada para o veículo considerando o movimento em todas as direções é dada por:

$$
M_{A}=\left[\begin{array}{cccccc}
X_{\dot{u}} & X_{\dot{v}} & X_{\dot{w}} & X_{\dot{p}} & X_{\dot{q}} & X_{\dot{r}} \\
Y_{\dot{u}} & Y_{\dot{v}} & Y_{\dot{w}} & Y_{\dot{p}} & Y_{\dot{q}} & Y_{\dot{r}} \\
Z_{\dot{u}} & Z_{\dot{v}} & Z_{\dot{w}} & Z_{\dot{p}} & Z_{\dot{q}} & Z_{\dot{r}} \\
K_{\dot{u}} & K_{\dot{v}} & K_{\dot{w}} & K_{\dot{p}} & K_{\dot{q}} & K_{\dot{r}} \\
M_{\dot{u}} & M_{\dot{v}} & M_{\dot{w}} & M_{\dot{p}} & M_{\dot{q}} & M_{\dot{r}} \\
N_{\dot{u}} & N_{\dot{v}} & N_{\dot{w}} & N_{\dot{p}} & N_{\dot{q}} & N_{\dot{r}}
\end{array}\right]
$$

A forma aproximadamente simétrica do casco do veículo (nos planos $x y$ e $x z$ ) sugere que sejam eliminados alguns coeficientes de valor ínfimo, obtendo-se assim a matriz de massa 
adicionada para o veículo estudado:

$$
M_{A}=\left[\begin{array}{cccccc}
X_{\dot{u}} & 0 & 0 & 0 & 0 & 0 \\
0 & Y_{\dot{v}} & 0 & Y_{\dot{p}} & 0 & Y_{\dot{r}} \\
0 & 0 & Z_{\dot{w}} & 0 & Z_{\dot{q}} & 0 \\
0 & K_{\dot{v}} & 0 & K_{\dot{p}} & 0 & K_{\dot{r}} \\
0 & 0 & M_{\dot{w}} & 0 & M_{\dot{q}} & 0 \\
0 & N_{\dot{v}} & 0 & N_{\dot{p}} & 0 & N_{\dot{r}}
\end{array}\right]
$$

Da expressão (3.25) derivam-se os termos cruzados de massa adicionada (IMLAY, 1961).

$$
\begin{array}{llll}
X_{w q}=Z_{\dot{w}} & X_{q q}=Z_{\dot{q}} & X_{v r}=-Y_{\dot{r}} & X_{r r}=-Y_{\dot{r}} \\
X_{r p}=-Y_{\dot{p}} & Y_{u r}=X_{\dot{u}} & Y_{w p}=-Z_{\dot{w}} & Y_{p q}=-Z_{\dot{q}} \\
Z_{u q}=-X_{\dot{u}} & Z_{v p}=Y_{\dot{v}} & Z_{r p}=Y_{\dot{r}} & Z_{r p}=Y_{\dot{r}} \\
Z_{p p}=Y_{\dot{p}} & & & \\
& & & \\
K_{v w}=-\left(Y_{\dot{v}}-Z_{\dot{w}}\right) & K_{w r}=-\left(Y_{\dot{r}}+Z_{\dot{q}}\right) & K_{v q}=\left(Z_{\dot{r}}+Z_{\dot{q}}\right) & K_{p q}=K_{\dot{r}} \\
M_{u w a}=-\left(Z_{\dot{w}}-X_{\dot{u}}\right) & M_{v p}=-Y_{\dot{r}} & M_{r p}=\left(K_{\dot{p}}-N_{\dot{r}}\right) & M_{u q}=-Z_{\dot{q}} \\
M_{p p}=-K_{\dot{r}} & M_{r r}=K_{\dot{r}} & N_{u v a}=-\left(X_{\dot{u}}-Y_{\dot{v}}\right) & N_{w p}=Z_{\dot{q}} \\
N_{p q}=-\left(K_{\dot{p}}-M_{\dot{q}}\right) & N_{u r}=Y_{\dot{r}} & N_{v q}=-Y_{\dot{p}} & N_{q r}=-K_{\dot{r}}
\end{array}
$$

Na equação (3.26) acima, os termos deixados de lado na modelagem do VSA foram: $Z_{r p}$, $Z_{p p}, K_{v w}, K_{w r}, K_{v q}, K_{p q}, M_{p p}, M_{r r}, M_{v r}, M_{v p}, N_{w p}, N_{v q}$ e $N_{q r}$.

\subsubsection{Resistência ao Avanço}

Trata-se de uma força que age contra o movimento do submersível no meio fluido. Este esforço dissipativo pode ser de dois tipos: arrasto de fricção, gerado por tensões tangenciais de natureza viscosa entre o fluido e o casco do VSA, e arrasto de forma ou de pressão, causado por diferenças de pressões normais à superfície do corpo à jusante e à montante do veículo. Em Canhetti (1998), o arrasto hidrodinâmico é modelado pelo termo $R_{v}$ seguinte:

$$
R v=-0.2478 u^{3}+24.98 u^{2}+8.5217 u
$$

O comportamento desta força pode ser observado na figura 3.3.

Para o VSA, foi modelado o arrasto hidrodinâmico que produzem os lemes através dos coeficientes $X_{\delta_{r} \delta r}, X_{\delta_{s} \delta s}$ (na direção de avanço) e $K_{\delta_{r}}$ (na direção de balanço). Também foram modelados os arrastos nas direções de deriva $Y_{p|p|}$ e $Y_{v|v| R}$, caturro $M_{w|w| R}$ e guinada $N_{v|v| R}$ 


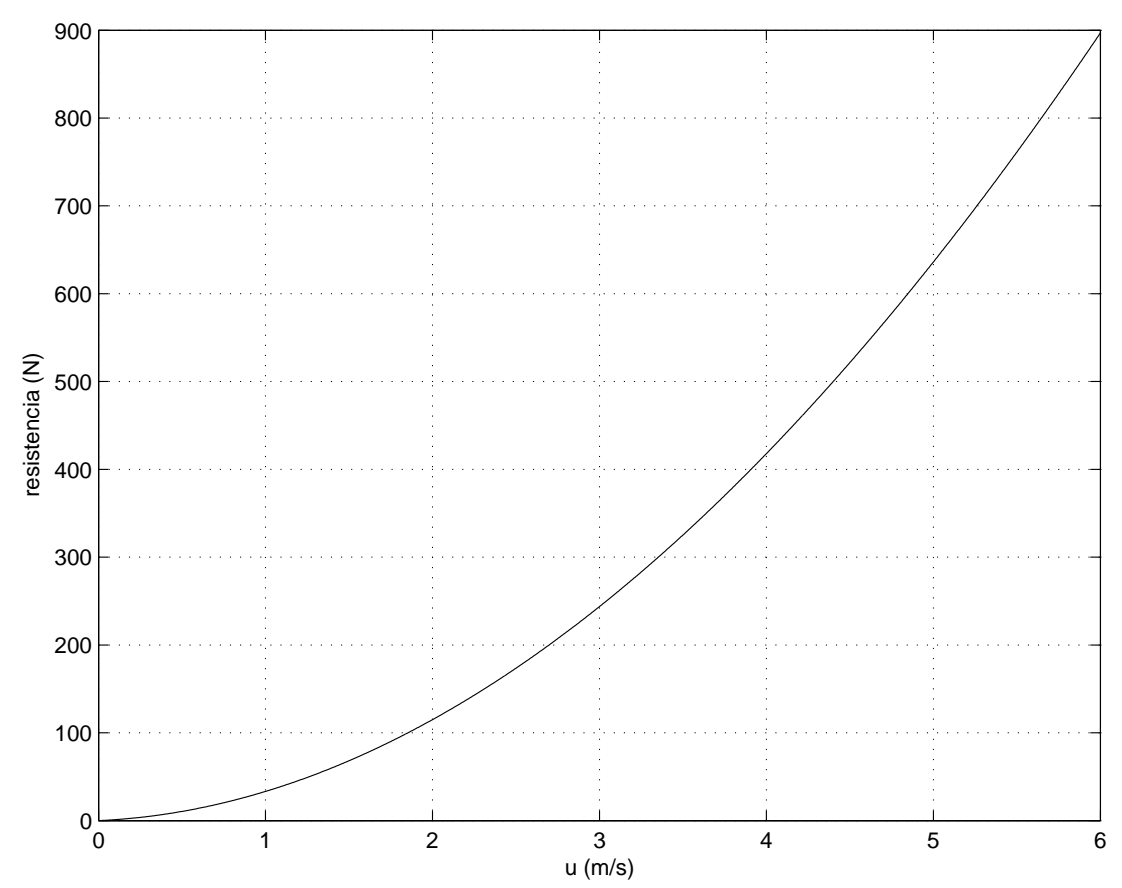

Figura 3.3: Resistência de avanço para o VSA.

\subsubsection{Propulsor}

O empuxo gerado pelos propulsores são de extrema importância para o movimento e para o controle dos veículos submersíveis. Aplicando forças no fluido através da rotação de propulsores, o veículo sofre forças de reação aplicadas pelo fluido e, assim, consegue movimentá-lo no meio submarino. Em Feldman (1979), a força propulsora é modelada pelo termo $T_{p}$ da equação (3.44) adiante, e o torque ou momento gerado pelos propulsores é modelado pelo termo $Q_{p}$ da equação (3.47) adiante. O uso de propulsores contrarotativos é usual em projetos de torpedo, cuja vantagem principal é a diminuição de efeitos indesejáveis do torque motor e hidrodinâmico atuantes no sistema. Isto leva a uma diminuição na solicitação de lemes e atuadores, e portanto reduz-se a resistência ao avanço. Neste trabalho foram adotados os dados do modelo livre desenvolvido pela Marinha do Brasil, nos trabalhos de Sbragio (1995) e Domiciniano (1996). Define-se coeficiente de avanço do propulsor por :

$$
J=\frac{U_{p}}{n_{p} D}
$$

onde $U_{p}$ é a velocidade do fluido que atravessa o propulsor $(\mathrm{m} / \mathrm{s}), D$ é o diâmetro do propulsor $(\mathrm{m})$ e $n_{p}$ é a velocidade de rotação do propulsor (rpm).

$U_{p}=U\left(1-w_{e}\right)$, onde o parâmetro $U$ é a velocidade do veículo e $w_{e}$ é o coeficiente de esteira, que relaciona a velocidade do veículo com a velocidade da água. O valor de $w_{e}$ para o projeto do VSA foi de 0,1 , o que indica que a velocidade da água que entra no 


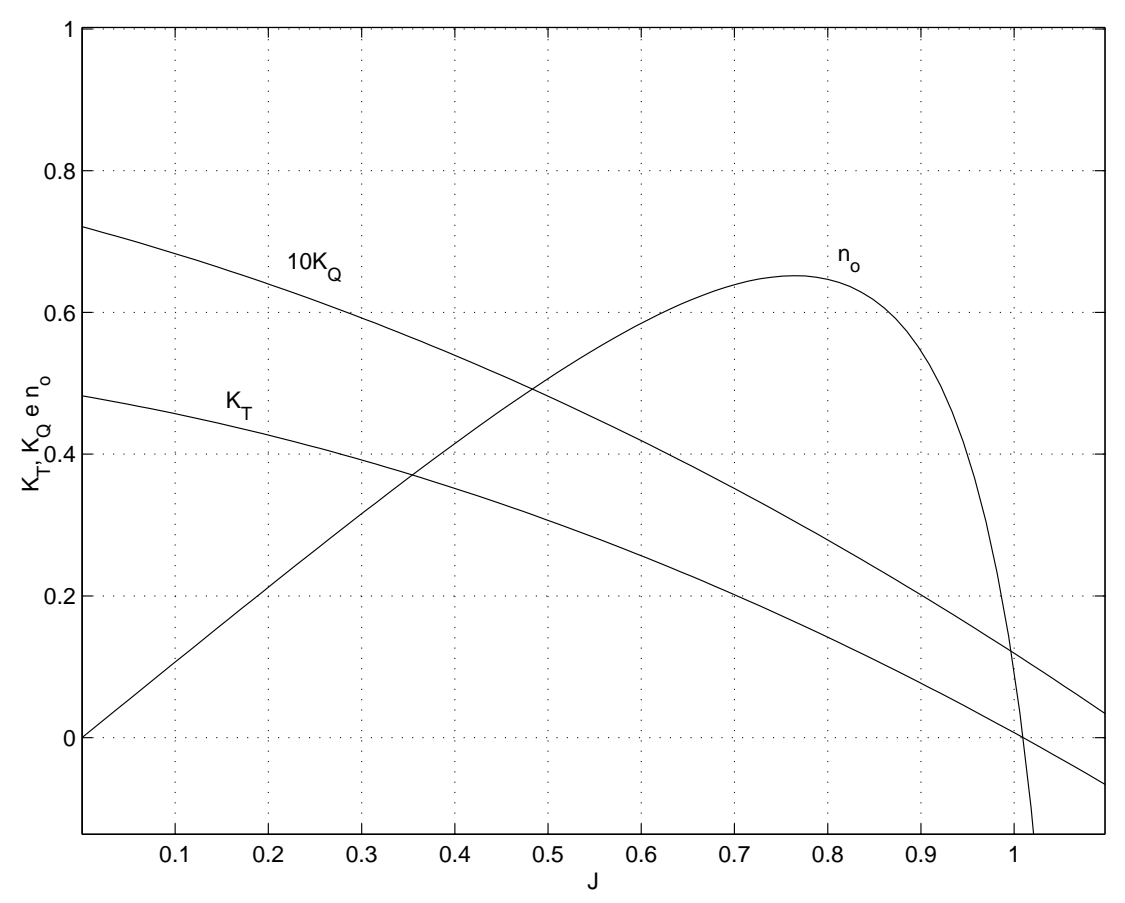

Figura 3.4: Curvas características do propulsor para o veículo submersível autônomo $K_{T}, K_{Q}$ e $\eta_{o}$.

propulsor é apenas $90 \%$ da velocidade do veículo.

O funcionamento do propulsor na água pode ser caracterizado por dois parâmetros adimensionais: o coeficiente de empuxo e o coeficiente de torque respectivamente dados por:

$$
\begin{aligned}
K_{T} & =\frac{T_{p}}{\rho \eta_{p}^{2} D^{4}} \\
K_{Q} & =\frac{Q_{p}}{\rho \eta_{p}^{2} D^{5}}
\end{aligned}
$$

Estes coeficientes são parametrizados em função do coeficiente de avanço (J), e modelados pelas seguintes equações:

$$
\begin{aligned}
& K_{T}=-0.2482 J^{2}-0.2272 J+0.4823 \\
& K_{Q}=-0.0247 J^{2}-0.0355 J+0.0721
\end{aligned}
$$

O desempenho do propulsor é definido por:

$$
\eta_{o}=\frac{T_{p} U}{2 \pi \eta_{o} Q_{p}}=\frac{J K_{T}}{2 \pi K_{Q}}
$$

A figura 3.4 apresenta as curvas características do propulsor $K_{T}, K_{Q}$ e $\eta_{o}$ para o VSA 
em função de $J$.

\subsubsection{Esforços de Sustentação}

Esforços de sustentação são esforços normais ao movimento do veículo, que surgem quando o submarino se desloca no fluido. Esses esforços são dependentes da geometria do casco e lemes do submersível e, também, da velocidade de avanço do veículo. Os esforços de sustentação são modelados de acordo com as formulas empíricas bem conhecidas (HOERNER, 1992):

$$
L=-\frac{1}{2} \rho A_{p} C_{l} u^{2}
$$

onde L é o esforço de sustentação, $\rho$ é a densidade do fluido, $A_{p}$ é a área projetada do casco do veiculo, $u$ é a velocidade do veículo e $C_{l}$ é o coeficiente de sustentação. A partir da fórmula (3.32) encontram-se os coeficientes de força e momento tanto para o corpo rígido quanto para os lemes atuadores.

Segundo Prestero (2001), sempre que se garanta a condição estabelecida por Hoerner (1992) $\left(6,7 \leq \frac{l}{d} \leq 11\right)$ e para ângulos de ataque pequenos $\left(\tan \left(\frac{w}{u}\right) \approx \frac{w}{u}\right.$ e $\left.\tan \left(\frac{v}{u}\right) \approx \frac{v}{u}\right)$, os esforços de sustentação gerados pelo veículo podem se escrever como segue:

$$
L_{Z}=-\frac{1}{2} \rho A_{p} C_{Z} u w \quad e \quad L_{Y}=-\frac{1}{2} \rho A_{p} C_{Y} u v
$$

$L_{Z}$ e $L_{Y}$ são os esforços de sustentação relativos ao eixo $z$ e $y$, respectivamente; $C_{Z}$ é $C_{Y}$ são os coeficientes de sustentação associados a ambos esforços. $u, v$ e $w$ são as velocidades de avanço, deriva e arfagem do veículo, respectivamente.

De (3.33) resulta $Z_{w}$ e $Y_{v}$, coeficientes usados no modelo matemático do VSA:

$$
Z_{w}=-\frac{1}{2} \rho A_{p} C_{Z} \quad \text { e } \quad Y_{v}=-\frac{1}{2} \rho A_{p} C_{Y}
$$

Dada a geometria do VSA, é importante adicionar mais dois coeficientes hidrodinâmicos $M_{w}$ e $N_{v}$ relativos ao momento Munk, que obedecem à ação da força viscosa do fluido e às massas adicionais:

$$
\begin{gathered}
M_{w}=M_{u w a}+Z_{w}\left(-x_{z e r o}-0,65 l\right) \\
N_{v}=N_{u v a}+Y_{w}\left(-x_{z e r o}-0,65 l\right)
\end{gathered}
$$

$M_{u w a}$ e $N_{u v a}$ resultam da equação (3.26) e representam o momento no corpo rígido do veículo, $x_{z e r o}$ é a posição da popa do VSA relativamente à origem do sistema de coordenadas móveis. A força viscosa está localizada a 0,65 do comprimento total do veículo 
(PRESTERO, 2001).

Os esforços de sustentação produzidos pelos lemes atuadores também podem ser separados em forças e momentos. Assim, utilizando-se a equação (3.32), para um leme (fólio) as fórmulas empíricas para os esforços de sustentação são dadas como seguem:

$$
\begin{aligned}
L_{f} & =\frac{1}{2} \rho C_{l f} S_{f} \delta_{e} v_{e}^{2} \\
M_{f} & =x_{f} L_{f}
\end{aligned}
$$

onde $C_{l f}$ é o coeficiente de sustentação do fólio, $S_{f}$ é a área plana do folio, $\delta_{e}$ é o ângulo efetivo do fólio, $v_{e}$ é a velocidade efetiva do folio, $x_{f}$ é a posição do fólio relativamente ao sistema de coordenadas móveis. $C_{l f}$ é definido em função do ângulo de ataque (HOERNER, 1992):

$$
C_{l f}=\frac{d C_{l f}}{d \alpha}=\frac{2 \pi}{1 / \alpha+2 / A R}
$$

onde AR é a razão de aspecto do fólio, dado pela fórmula seguinte:

$$
A R=b^{2} / S_{f}
$$

onde $b$ é a largura do fólio e $S_{f}$ é a área da superfície plana do fólio.

De maneira que, substituindo as expressões acima (3.37) e (3.38) na equação (3.36) obtêmse os esforços de sustentação dos lemes atuadores:

$$
\begin{aligned}
Y_{r} & =\frac{1}{2} \rho C_{l f} S_{f} u^{2} \delta_{r} \\
Z_{s} & =-\frac{1}{2} \rho C_{l f} S_{f} u^{2} \delta_{s} \\
M_{s} & =\frac{1}{2} \rho C_{l f} S_{f} x_{f} u^{2} \delta_{r} \\
N_{r} & =\frac{1}{2} \rho C_{l f} S_{f} x_{f} u^{2} \delta_{s}
\end{aligned}
$$

Finalmente, os coeficientes hidrodinâmicos para os esforços de sustentação dos lemes atuadores são:

$$
\begin{aligned}
Y_{\delta_{r}} & =\frac{1}{2} \rho C_{l f} S_{f} \\
Z_{\delta_{s}} & =-\frac{1}{2} \rho C_{l f} S_{f} \\
M_{\delta_{s}} & =\frac{1}{2} \rho C_{l f} S_{f} x_{f} \\
N_{\delta_{r}} & =\frac{1}{2} \rho C_{l f} S_{f} x_{f}
\end{aligned}
$$




\subsubsection{Esforços Restauradores}

O veículo experimenta forças e momentos hidrostáticos restauradores devido ao empuxo hidrostático $(B)$. Estas componentes estão em direções laterais e verticais relativos ao movimento do veículo submarino. Obviamente, o peso do VSA é $W=m g$ e o empuxo hidrostático $B=-\rho \nabla g$, onde $\rho$ representa a densidade do fluido e $\nabla$ representa o volume de fluido deslocado.

É necessário expressar essas forças e momentos em relação ao sistema de coordenadas fixo Oxyz, para isso se usa a seguinte matriz de transformação:

$$
\vec{f}_{G}\left(\eta_{2}\right)=J_{1}^{-1}\left[\begin{array}{c}
0 \\
0 \\
W
\end{array}\right] \quad \vec{f}_{B}\left(\eta_{2}\right)=J_{1}^{-1}\left[\begin{array}{l}
0 \\
0 \\
B
\end{array}\right]
$$

As forças hidrostáticas e momentos podem ser expressos por:

$$
\begin{array}{r}
\vec{F}_{H S}=\vec{f}_{G}-\vec{f}_{B} \\
\vec{M}_{H S}=\vec{r}_{G} \times \vec{f}_{G}-\vec{r}_{B} \times \vec{f}_{B}
\end{array}
$$

Estas equações levam a equações não lineares para as forças e momentos hidrostáticos, reproduzidas a seguir:

$$
\left\{\begin{array}{c}
X_{H S} \\
Y_{H S} \\
Z_{H S} \\
K_{H S} \\
M_{H S} \\
N_{H S}
\end{array}\right\}=\left\{\begin{array}{c}
-(W-B) \operatorname{sen} \theta \\
-(W-B) \cos \theta \operatorname{sen} \theta \\
-(W-B) \cos \theta \cos \phi \\
\left(y_{G} W-y_{B} B\right) \cos \theta \cos \phi-\left(z_{G} W-z_{B} B\right) \cos \theta \operatorname{sen} \phi \\
\left(x_{G} W-x_{B} B\right) \cos \theta \cos \phi-\left(z_{G} W-z_{B} B\right) \operatorname{sen} \theta \\
\left(x_{G} W-x_{B} B\right) \cos \theta \sin \phi-\left(y_{G} W-y_{B} B\right) \operatorname{sen} \theta
\end{array}\right\}
$$

\subsection{Modelo Não Linear do Veículo}

Na seção anterior apresentou-se a origem dos coeficientes hidrodinâmicos que compõem as forças e momentos sobre o veículo. As equações (3.16) e (3.23) podem ser expandidas o que leva ao conjunto de equações que modelam o movimento do veículo nos seis graus de liberdade. 
Avanço (translação na direção do eixo $x$ ):

$$
\begin{array}{r}
m\left[\dot{u}-v r+w q-x_{G}\left(q^{2}+r^{2}\right)+y_{G}(p q-\dot{r})+z_{G}(p r+\dot{q})\right] \\
=X_{q q} q^{2}+X_{r r} r^{2}+X_{v r} v r+X_{w q} w q+X_{\dot{u}} \dot{u}+ \\
X_{\delta_{r} \delta_{r}} u^{2} \delta_{r}^{2}+X_{\delta_{s} \delta_{s}} u^{2} \delta_{s}^{2}+T_{p}-\frac{R_{v}}{1-c_{t}}+X_{H S}
\end{array}
$$

Deriva (translação na direção do eixo y):

$$
\begin{aligned}
& m\left[\dot{v}-w p+u r-y_{G}\left(r^{2}+p^{2}\right)+z_{G}(q r-\dot{p})-x_{G}(q p+\dot{r})\right] \\
& =Y_{p|p|} p|p|+Y_{p q} p q+Y_{u r} u r+Y_{p} u p+Y_{w p} w p+Y_{v} u v+Y_{v|v| R} v \sqrt{v^{2}+w^{2}}+ \\
& Y_{\delta_{r}} u^{2} \delta_{r}+Y_{\dot{v}} \dot{v}+Y_{\dot{p}} \dot{p}+Y_{\dot{r}} \dot{r}+Y_{H S}
\end{aligned}
$$

Arfagem (translação na direção do eixo $z$ ):

$$
\begin{aligned}
& m\left[\dot{w}-u q+v p-z_{G}\left(p^{2}+q^{2}\right)+x_{G}(r p-\dot{q})+y_{G}(r q+\dot{p})\right] \\
& =Z_{q} u q+Z_{v p} v p+Z_{w} u w+Z_{\delta_{s}} u^{2} \delta_{s}+Z_{\dot{w}} \dot{w}+Z_{\dot{q}} \dot{q}+Z_{H S}
\end{aligned}
$$

Balanço (rotação ao redor do eixo $x$ ):

$$
\begin{array}{r}
I_{x} \dot{p}+\left(I_{z}-I_{y}\right) r q+m\left[y_{G}(\dot{w}+p v-q u)-z_{G}(\dot{v}+r u-p w)\right] \\
=K_{q r} q r+K_{p} u p+K_{r} u r+K_{w p} w p+K_{v r} u v+K_{\delta_{r}} u^{2} \delta_{r}+Q_{p}+ \\
K_{\dot{v}} \dot{v}+K_{\dot{r}} \dot{r}+K_{\dot{p}} \dot{p}+K_{H S}
\end{array}
$$

Caturro (rotação ao redor do eixo $y$ ):

$$
\begin{array}{r}
I_{y} \dot{q}+\left(I_{x}-I_{z}\right) r p+m\left[z_{G}(\dot{u}+q w-r v)-x_{G}(\dot{w}+p v-q u)\right] \\
=M_{r p} r p+M_{q} u q+M_{*} u^{2}+M_{w} u w+M_{w|w| R} w \sqrt{v^{2}+w^{2}}+ \\
M_{\delta_{s}} u^{2} \delta_{s}+M_{\dot{w}} \dot{w}+M_{\dot{q}} \dot{q}+M_{H S}
\end{array}
$$

Guinada (rotação ao redor do eixo $z$ ):

$$
\begin{array}{r}
I_{z} \dot{r}+\left(I_{y}-I_{x}\right) q p+m\left[x_{G}(\dot{v}+r u-p w)-y_{G}(\dot{u}+q w-r v)\right] \\
=N_{p q} p q+N_{p} u p+N_{r} u r+N_{v} u v+N_{v|v| R} v \sqrt{v^{2}+w^{2}}+ \\
N_{\delta_{r}} u^{2} \delta_{r}+N_{\dot{v}} \dot{v}+N_{\dot{p}} \dot{p}+N_{\dot{r}} \dot{r}+N_{H S}
\end{array}
$$




\subsection{Modelo Dinâmico Vetorial}

Dada a complexidade do modelo e sua natureza altamente não-linear, é imprescindível o uso de métodos de integração numérica para a solução do sistema de equações obtido.

Realizam-se, a seguir, modificações nas equações que governam o movimento do VSA, com o fim de facilitar a manipulação computacional.

Após transformações algébricas simples, as equações (3.44)-(3.49) são colocadas na forma usual da Segunda Lei de Newton: onde $\mathbf{M}$ é a matriz de massas inércias e massas adicionais, $v$ é o vetor velocidade do veículo em relação ao sistema de coordenadas móveis (equação 3.3), e F é o vetor de forças e momentos totais que atuam no veículo:

$$
\mathbf{M} \dot{v}=\mathbf{F}
$$

onde

$$
\begin{gathered}
\mathbf{M}=\left[\begin{array}{cccccc}
m-X_{\dot{u}} & 0 & 0 & 0 & m z_{G} & -m z_{G} \\
0 & m-Y_{\dot{v}} & 0 & -Y_{\dot{p}}-m_{G} & 0 & -Y_{\dot{r}}-m x_{G} \\
0 & 0 & m-Z_{\dot{w}} & m y_{G} & -m x_{G}-Z \dot{q} & 0 \\
0 & -m z_{G}-K_{\dot{v}} & m y_{G} & I_{x}-K_{\dot{p}} & 0 & -K_{\dot{r}} \\
m x_{G} & 0 & -m x_{G}-M_{\dot{w}} & 0 & I_{y}-M_{\dot{q}} & 0 \\
-m z_{G} & m x_{G}-N_{\dot{v}} & 0 & -N_{\dot{p}} & 0 & I_{z}-N_{\dot{r}}
\end{array}\right] \\
\mathbf{F}=\left[\begin{array}{llllll}
X & Y & Z & M & N & K
\end{array}\right]^{T} \\
v=\left[\begin{array}{llllll}
u & v & w & p & q & r
\end{array}\right]^{T}
\end{gathered}
$$




$$
\begin{aligned}
X= & m\left[v r-w q+x_{G}\left(q^{2}+r^{2}\right)-y_{G} p q-z_{G} p r\right]+ \\
& X_{q q} q^{2}+X_{r r} r^{2}+X_{v r} v r+X_{w q} w q+X_{\dot{u}} \dot{u}+ \\
& X_{\delta_{r} \delta_{r}} u^{2} \delta_{r}^{2}+X_{\delta_{s} \delta_{s}} u^{2} \delta_{s}^{2}+T_{p}-\frac{R_{v}}{1-c_{t}}+X_{H S} \\
Y= & m\left[w p-u r+y_{G}\left(r^{2}+p^{2}\right)-z_{G} q r+x_{G} q p\right]+ \\
& Y_{p|p|} p|p|+Y_{p q} p q+Y_{u r} u r+Y_{p} u p+Y_{w p} w p+ \\
& Y_{v} u v+Y_{v|v| R} v \sqrt{v^{2}+w^{2}}+Y_{\delta_{r}} u^{2} \delta_{r}+Y_{H S} \\
Z= & m\left[u q-v p+z_{G}\left(p^{2}+q^{2}\right)-x_{G} r p-y_{G} r q\right]+ \\
& Z_{q} u q+Z_{v p} v p+Z_{w} u w+Z_{\delta_{s}} u^{2} \delta_{s}+Z_{H S} \\
K= & \left(I_{y}-I_{z}\right) r q-m\left[y_{G}(p v-q u)-z_{G}(r u-p w)\right]+ \\
& K_{q r} q r+K_{p} u p+K_{r} u r+K_{w p} w p+K_{v r} u v+K_{\delta_{r}} u^{2} \delta_{r}+ \\
& Q_{p}+K_{H S} \\
M= & -I_{y} \dot{q}+\left(I_{z}-I_{x}\right) r p-m\left[z_{G}(q w-r v)-x_{G}(p v-q u)\right]+ \\
& M_{r p} r p+M_{q} u q+M_{*} u^{2}+M_{w} u w+ \\
& M_{w|w| R} w \sqrt{v^{2}+w^{2}}+M_{\delta_{s}} u^{2} \delta_{s}+M_{H S} \\
N= & -I_{z} \dot{r}+\left(I_{x}-I_{y}\right) q p-m\left[x_{G}(r u-p w)-y_{G}(q w-r v)\right]+ \\
& N_{p q} p q+N_{p} u p+N_{r} u r+N_{v} u v+ \\
& N_{v|v| R} v \sqrt{v^{2}+w^{2}}+N_{\delta_{r}} u^{2} \delta_{r}+N_{H S}
\end{aligned}
$$

Assim, a expressão matricial da Segunda Lei de Newton (3.50), pode ser reescrita como:

$$
\dot{v}=\mathbf{M}^{-1} \mathbf{F}
$$

que pode facilmente ser colocada na forma usual de equações de estado:

$$
\begin{aligned}
\dot{\mathbf{x}}(t) & =\mathbf{f}(\mathbf{x}(t), \mathbf{u}(t)) \text { (equação de evolução de estados) } \\
\mathbf{y}(t) & =\mathbf{g}(\mathbf{x}(t), \mathbf{u}(t)) \text { (equação de saídas instantâneas do sistema) }
\end{aligned}
$$

onde $\mathbf{x}(t) \in \mathbb{R}^{12}$ é o vetor de estados; $\mathbf{u}(t) \in \mathbb{R}^{3}$ é o vetor de entradas; $\mathbf{y}(t) \in \mathbb{R}^{4}$ é o vetor de estados medidos; $\mathbf{f}(.) \in \mathbb{R}^{12}$ é uma função não linear vetorial de estado; $\mathbf{g}(.) \in \mathbb{R}^{4}$ é uma função vetorial contínua é limitada.

A equação (3.56) resulta das Equações Diferenciais Ordinárias (EDOs) que definem as equações dinâmicas do veículo (3.55) e das equações de sua cinemática (3.2) e (3.5). A equação (3.57) define os estados observados do sistema.

Para facilidade de leitura, os vetores de estado, de entrada e saída são explicitados a seguir: 


$$
\mathbf{x}(t)=\left[\begin{array}{c}
u(t) \\
v(t) \\
w(t) \\
p(t) \\
q(t) \\
r(t) \\
x(t) \\
y(t) \\
z(t) \\
\phi(t) \\
\theta(t) \\
\psi(t)
\end{array}\right] \quad \mathbf{u}(t)=\left[\begin{array}{c}
\eta(t) \\
\delta_{r}(t) \\
\delta_{s}(t)
\end{array}\right] \quad \mathbf{y}(t)=\left[\begin{array}{c}
\phi(t) \\
\theta(t) \\
\dot{\psi}(t) \\
\dot{z}(t)
\end{array}\right]
$$

O modelo não linear do VSA é invariante no tempo. A não linearidade inclui um forte acoplamento dinâmico entre suas variáveis e na matriz de transformação de coordenadas $(J)$.

\subsection{Linearização}

É necessário linearizar o modelo do veículo para as leis de controle que se vão aplicar aqui. A partir das equações (3.56) e (3.57), obtém-se, inicialmente, a solução em regime permanente $\mathbf{x}_{o}(t), \mathbf{u}_{o}(t)$ e $\mathbf{y}_{o}(t)$ (solução nominal do sistema), que satisfaçam às equações não lineares do veículo.

Para linearização utilizou-se o método das perturbações. Assim, o vetor de estados, o vetor de entradas e o vetor de condições iniciais são substituídos respectivamente por:

$$
\begin{aligned}
\mathbf{x}(t) & =\mathbf{x}(t)+\delta \mathbf{x}(t) \\
\mathbf{u}(t) & =\mathbf{u}(t)+\delta \mathbf{u}(t) \\
\mathbf{x}_{o}(t) & =\mathbf{x}_{o}(t)+\delta \mathbf{x}_{o}(t)
\end{aligned}
$$

Substituindo as variáveis perturbadas nas equações (3.56) e (3.57) e expandindo em 
Série de Taylor de primeira ordem para o caso multivariável, obtém-se o seguinte sistema:

$$
\begin{aligned}
& \delta \dot{\mathbf{x}}(t)=\left[\frac{\partial \mathbf{f}}{\partial \mathbf{x}}\right]_{\mathbf{x}_{o}} \delta \mathbf{x}(t)+\left[\frac{\partial \mathbf{f}}{\partial \mathbf{x}}\right]_{\mathbf{u}_{o}} \delta \mathbf{u}(t) \\
& \delta \mathbf{y}(t)=\left[\frac{\partial \mathbf{g}}{\partial \mathbf{x}}\right]_{\mathbf{x}_{o}} \delta \mathbf{x}(t)+\left[\frac{\partial \mathbf{g}}{\partial \mathbf{x}}\right]_{\mathbf{u}_{o}} \delta \mathbf{u}(t)
\end{aligned}
$$

Onde a matriz $\left[\frac{\partial \mathbf{f}}{\partial \mathbf{x}}\right]_{\mathbf{x}_{o}}$ de ordem $n \times n$ é o Jacobiano, ou as derivadas parciais de $\mathbf{f}$ com respeito a $\mathbf{x}$. As outras matrizes jacobianas da equação (3.60) são obtidas de maneira similar.

O modelo linearizado na equação (3.60) será invariante no tempo, se a solução nominal for constante, isto é, se a solução nominal corresponder a um ponto de equilíbrio, tal que:

$$
\begin{array}{r}
\mathbf{f}(\mathbf{x}(t), \mathbf{u}(t))=\mathbf{A x}(t)+\mathbf{B u}(t) \\
\mathbf{g}(\mathbf{x}(t), \mathbf{u}(t))=\mathbf{C x}(t)+\mathbf{D} \mathbf{u}(t)
\end{array}
$$

Onde:

$$
\begin{array}{ll}
\mathbf{A}=\left[\frac{\partial \mathbf{f}}{\partial \mathbf{x}}\right]_{\mathbf{x}_{o}} \quad \mathbf{B}=\left[\frac{\partial \mathbf{f}}{\partial \mathbf{u}}\right]_{\mathbf{u}_{o}} \\
\mathbf{C}=\left[\frac{\partial \mathbf{g}}{\partial \mathbf{x}}\right]_{\mathbf{x}_{o}} \quad \mathbf{D}=\left[\frac{\partial \mathbf{g}}{\partial \mathbf{u}}\right]_{\mathbf{u}_{o}}
\end{array}
$$

As matrizes A, B, C e D são as derivadas parciais do modelo não linear, avaliadas num ponto de equilíbrio: $\mathbf{x}_{o}=\left[\begin{array}{llllllllllll}u_{o} & v_{o} & w_{o} & p_{o} & q_{o} & r_{o} & x_{o} & y_{o} & z_{o} & \phi_{o} & \theta_{o} & \psi_{o}\end{array}\right]^{T}$, $\mathbf{u}_{o}=\left[\begin{array}{lll}\eta_{o} & \delta_{r_{o}} & \delta_{s_{o}}\end{array}\right]^{T}$. 


\section{Lei de Controle}

\subsection{Introdução}

No presente capítulo apresenta-se a teoria de controle $\mathcal{H}_{\infty}$ utilizada neste trabalho. O material de referência são os trabalhos de Skogestad e Postlethwaite (1996), Donha (2003) e Zhou (2000). Todos estes textos adotam como referências principais artigos, apostilas e livros clássicos onde o fundamento matemático está bem exposto como: Zames (1979), Francis (1988) e Doyle, Francis e Tannenbaum (1990).

O projeto de qualquer controlador envolve basicamente duas tarefas principais: a estabilidade e o ajuste (sintonia) de seus parâmetros para alcançar um desempenho considerado 'ótimo' de acordo com algum critério (índice de desempenho), de forma a satisfazer especificações estabelecidas pelo projetista Donha (2003).

A robustez é uma característica importante de sistemas de controle por pelo menos duas razões. Em primeiro lugar, deve ser preocupação permanente de todo projetista que os sistemas de controle funcionem satisfatoriamente, ainda que as condições de operação sejam distintas daquelas consideradas no modelo de projeto nominal. Em segundo lugar, as condições de robustez podem ser utilizadas com o objetivo de se adotar um modelo de projeto intencionalmente simplificado, não só para facilitar a análise, como também por seu impacto sobre a complexidade do controlador resultante (CRUZ, 1996).

Se o controlador consegue manter a estabilidade para um certo conjunto de condições distinto da nominal, diz-se que o sistema controlado apresenta Estabilidade Robusta (ER) para este conjunto. Analogamente, se o desempenho do sistema controlado não é degradado significativamente, de forma que as especificações do projeto ainda sejam atendidas para uma familia de sistemas incerta, oriunda de um único sistema nominal, diz-se que o sistema apresenta Desempenho Robusto (DR) (ZHOU; DOYLE; GLOVER, 1995).

Em resumo, a estabilidade e desempenho nominais são definidos para plantas nominais, enquanto que ER e DR são relativos a uma familia de plantas, geralmente originárias da planta nominal, e sujeitas a incertezas (de modelo, distúrbio e ruídos) que devem incluir as incertezas da pior espécie (worst case uncertainty) para o problema considerado. 


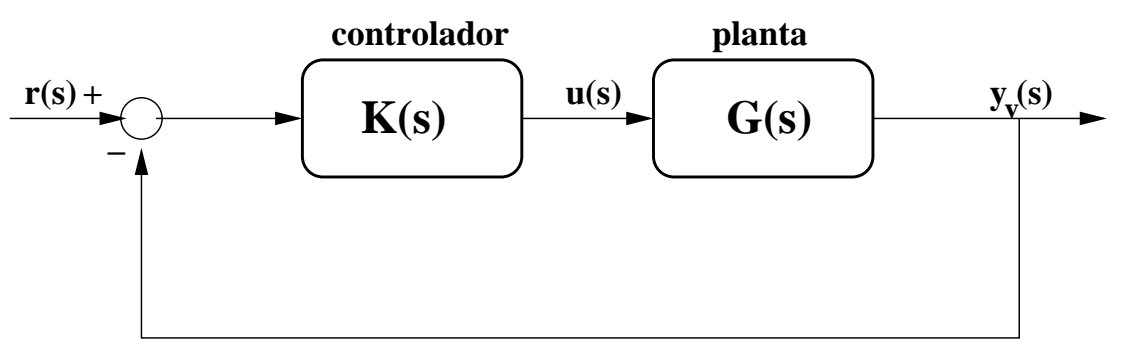

Figura 4.1: Controlador Clássico - SISO.

As teorias de controle apresentadas neste capítulo facilitaram a síntese do controlador com estabilidade e desempenho robustos considerando incertezas não-estruturadas no modelo do VSA. A grande vantagem adicional é levar em conta distúrbios e ruídos presentes no sistema, já na fase de síntese.

\subsection{Abordagem de Projeto}

O processo geral de projeto do controlador para um sistema de controle é basicamente uma iteração entre a síntese de um controlador e a avaliação de seu desempenho. Se o desempenho não é satisfatório, então é preciso ajustar-se algum parâmetro do controlador, por exemplo, modificando-se o ganho proporcional em um controlador PID, ou ajustar algum fator de ponderação no índice de desempenho escolhido, como é o caso de controles ótimos. Para facilitar o entendimento, vai se usar a representação de diagrama de blocos do sistema escalar com realimentação da figura 4.1. Nessa figura $K(s)$ e $G(s)$ representam, respectivamente, as transformadas de Laplace da dinâmica temporal do controlador e da planta ou processo, $r(s)$ é a transformada de Laplace do sinal de controle e $y_{v}(s)$ é transformada de Laplace do sinal de saída verdadeiro do sistema.

\subsection{Representação do Sistema Dinâmico Linear}

Como vimos no capitulo (3), após a linearização ao redor de uma posição de equilíbrio, o sistema pode ser representado na forma convencional de equações de estados, como segue-se:

$$
\begin{aligned}
& \dot{x}(t)=A x(t)+B u(t), x\left(t_{o}\right)=x_{o} \\
& y(t)=C x(t)+D u(t)
\end{aligned}
$$


onde $x(t) \in \mathbb{R}^{8}$ é o estado do sistema; $x\left(t_{o}\right)$ é a condição inicial; $u(t) \in \mathbb{R}^{2}$ é a entrada do sistema e $y(t) \in \mathbb{R}^{4}$ é a saída ou o estado observado.

As matrizes $A, B, C, D$, de dimensões adequadas, são compostas de constantes reais.

Usando-se a notação usual, exprimem-se as equações acima por:

$$
\left[\begin{array}{l}
\dot{x}(t) \\
y(t)
\end{array}\right]=\left[\begin{array}{ll}
A & B \\
C & D
\end{array}\right]\left[\begin{array}{l}
x(t) \\
u(t)
\end{array}\right]
$$

A resposta no tempo do sistema dinâmico pode ser determinada por:

$$
\begin{aligned}
& x(t)=e^{A\left(t-t_{o}\right)} x\left(t_{o}\right)+\int_{t_{o}}^{t} e^{A(t-\tau)} B u(\tau) d \tau \\
& y(t)=C x(t)+D u(t)
\end{aligned}
$$

Aplicando a transformada de Laplace ao sistema de equações (4.1), para condições iniciais quiescentes, pode-se determinar a matriz de transferência de $U(s)$ para $Y(s)$ definida por:

$$
Y(S)=G(S) U(s)
$$

obtendo-se:

$$
G(s)=C(s I-A)^{-1} B+D
$$

Nas teorias avançadas de controle é comum o uso da seguinte notação para representar a matriz de transferência $G(s)$, cuja realização no espaço de estado é dada pela quádrupla $(A, B, C, D)$ :

$$
G(s)=C(s I-A)^{-1} B+D \triangleq\left[\begin{array}{l|l}
A & B \\
\hline C & D
\end{array}\right]
$$

Em controle avançado, pode-se distinguir entre as variáveis de estado controladas (z) e variáveis de estado observadas $(y)$, já que nem sempre $z \equiv y$. A notação no espaço de estados fica, então:

$$
\begin{aligned}
\dot{x}(t) & =A x(t)+B_{1} w(t)+B_{2} u(t), x\left(t_{o}\right)=x_{o} \\
z(t) & =C_{1} x(t)+D_{11} w(t)+D_{12} u(t) \\
y(t) & =C_{2} x(t)+D_{21} w(t)+D_{22} u(t)
\end{aligned}
$$

Então:

$$
G(s)=\left[\begin{array}{ll}
G_{11}(s) & G_{12}(s) \\
G_{21}(s) & G_{22}(s)
\end{array}\right]=\left[\begin{array}{c|cc}
A & B_{1} & B_{2} \\
\hline C_{1} & D_{11} & D_{12} \\
C_{2} & D_{21} & D_{22}
\end{array}\right]
$$


onde $w(t)$ é o vetor de sinais exógenos e ruídos.

\subsection{Controlabilidade e Observabilidade}

Esta seção expõe dois conceitos importantes na teoria de sistemas lineares.

Definição 4.1 (Controlabilidade) O sistema dinâmico descrito em (4.7) ou o par $\left(A, B_{2}\right)$ é controlável se, para qualquer estado inicial $x(0)=x_{0}$, qualquer $t_{1}>0$ e qualquer estado final $x_{1}$, existe uma entrada $u(t)$ tal que $x\left(t_{1}\right)=x_{1}$. De outra maneira o sistema será não controlável.

Uma maneira simples de verificar a controlabilidade de um sistema é a seguinte: dado o par de matrizes $\left(A, B_{2}\right)$, o sistema é controlável se e somente se a matriz de controlabilidade

$$
\mathcal{C} \triangleq\left[\begin{array}{lllll}
B_{2} & A B_{2} & A^{2} B_{2} & \ldots & A^{n-1} B_{2}
\end{array}\right]
$$

é de posto $n$, ie, existe pelo menos um determinante não nulo de ordem $n$.

Definição 4.2 (Observabilidade) O sistema dinâmico descrito em (4.7) ou o par $\left(A, C_{2}\right)$ é observável se, para qualquer tempo $t_{1}>0$, o estado inicial $x(0)=x_{0}$ pode ser determinado a partir da história no tempo da entrada $u(t)$ e a saída y $(t)$ no intervalo $\left[0, t_{1}\right]$. De outra maneia o sistema, ou o par $(A, C)$ é não observável.

Uma maneira simples de verificar a observabilidade de um sistema é a seguinte: dado o par de matrizes $\left(A, C_{2}\right)$, o sistema é observável se a matriz de observabilidade

$$
\mathcal{O} \triangleq\left[\begin{array}{lllll}
C_{2} & C_{2} A & C_{2} A^{2} & \ldots & C_{2} A^{n-1}
\end{array}\right]^{T}
$$

é de posto $n$, ie., existe pelo menos um determinante não nulo de ordem $n$.

Em resumo, a controlabilidade de um sistema indica a possibilidade de transferir o sistema de um estado qualquer à origem (estado nulo), num intervalo finito de tempo, pela aplicação de uma ação de controle conveniente. Por outro lado, a observabilidade indica a possibilidade de se determinar os componentes do estado do sistema através de observações da saída do sistema.

Em controle avançado é importante apresentar-se também as seguintes duas definições que têm a ver com a capacidade de estabilizar e detectar o sistema (estabilizabilidade e a detectabilidade). 
Definição 4.3 O sistema, ou o par $\left(A, B_{2}\right)$ é estabilizável se existe um estado realimentado $u=F x$ que estabilize o sistema, i.e., $A+B_{2} F$ é estável.

Definição 4.4 O sistema, ou o par $\left(C_{2}, A\right)$ é detectável se $A+L C_{2}$ é estável para algum $L$.

\subsection{Ferramentas Analíticas}

Um objetivo muito importante de um sistema de controle, além de garantir a estabilidade, é satisfazer um certo conjunto de especificações relativas ao desempenho. Uma das maneiras de se verificar se as especificações foram satisfeitas, é através da magnitude de alguns sinais ou funções de transferências envolvidos. Por exemplo, a magnitude do sinal de erro de acompanhamento (tracking) do sistema deve ser pequena, enquanto que os sinais de controle não devem ser muito grandes, ou ainda a matriz de funções de transferência entre um distúrbio e a resposta do sistema deve ser pequena, etc. O desempenho de um sistema de controle pode ser determinado pela variação de certas funções de transferência com a freqüência. Esse método levou ao desenvolvimento de técnicas de controle muito utilizadas atualmente. Um destes métodos é o da formatação de malha-aberta (open-loop shaping), que será brevemente discutido. Um método alternativo de controle é oriundo da formatação em malha fechada de determinadas funções envolvendo a realimentação de sinais, como a função de sensibilidade, e a função de sensibilidade complementar. O método de formatação de malha fechada utilizado no presente trabalho é o da Sensibilidade Mista (mixed sensitivity).

\subsubsection{Valores Singulares}

A norma-2 ou euclidiana do vetor $u \in \mathbb{C}^{m}$ é dada por:

$$
\|u\|_{2}=\sqrt{u^{H} u}
$$

Define-se para o mesmo sistema a norma espectral da matriz $G \in \mathbb{R}^{n \times m}$, ou norma induzida pela norma euclidiana por:

$$
\|G\|_{2, i} \triangleq \sup _{\|u\|_{2}=1}\|G u\|_{2}
$$

Esta definição representa uma generalização do conceito de ganho para sistemas multivariáveis que graficamente pode ser visualizado na figura 4.2. 


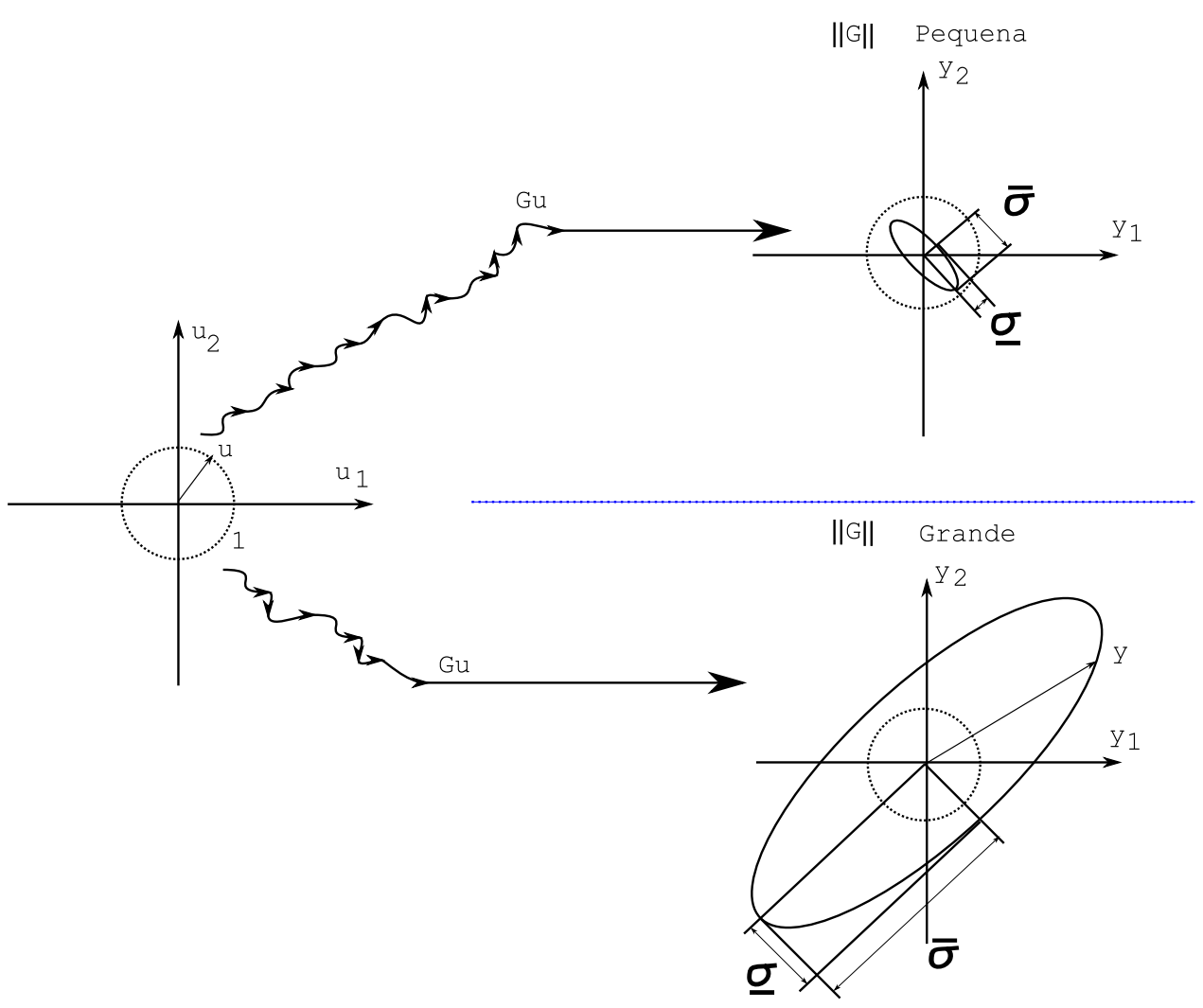

Figura 4.2: Matriz Pequena e Grande, valores singulares.

Do quociente de Raylegh (STRANG, 1988) resulta a seguinte definição para o máximo valor singular de uma matriz:

$$
\|G\|_{2, i}=\sqrt{\lambda_{M}\left(G^{H} G\right)}=\bar{\sigma}
$$

onde $\lambda_{M}$ representa o máximo autovalor do argumento, e $\bar{\sigma}$ é o máximo valor singular de $G$. De imediato, a expressão (4.13) sugere as seguintes definições:

$$
\sigma_{i}(G)=\sqrt{\lambda_{i}\left(G^{H} G\right)}
$$

onde $\sigma_{i}$ são os valores singulares de uma matriz em ordem decrescente:

$$
\bar{\sigma}=\sigma_{1} \geq \sigma_{2} \geq \ldots \geq \sigma_{p}=\underline{\sigma} .
$$

O número de valores singulares será determinado por $p=\min \{m, n\}, \bar{\sigma}$ e $\underline{\sigma}$ representam os valores singulares máximo e mínimo, respectivamente. 


\subsubsection{Norma $\mathcal{H}_{2}$ e $\mathcal{H}_{\infty}$}

Considere-se a representação de um sistema $G(s)$ contínuo por (estável), de dimensão $n \times m$, estritamente próprio, real e racional ${ }^{1}$.

Então, a norma- $\mathcal{H}_{2}$ de $G(s)$ é definida por:

$$
\|G\|_{2}=\left(\frac{1}{2 \pi} \operatorname{tr}\left\{\int_{-\infty}^{\infty}\left[G^{*}(j \omega) G(j \omega)\right]\right\}\right)^{1 / 2}
$$

A norma- $\mathcal{H}_{\infty}$ para $G(s)$ é definida por:

$$
\|G\|_{\infty}:=\sup _{\operatorname{Re}(s)>0} \bar{\sigma}[G(s)]=\sup _{\omega \in \mathbb{R}} \bar{\sigma}[G(j \omega)]
$$

\subsection{Incertezas}

As incertezas na planta ou modelo têm diversas origens (SKOGESTAD; POSTLETHWAITE, 1996), cabendo destacar:

1. a existência de erros nos valores dos parâmetros do modelo ou os valores dos parâmetros são desconhecidos;

2. os parâmetros no modelo linear podem variar devido a não linearidades ou variação do ponto de operação;

3. os erros associados aos instrumentos de medição e

4. a estrutura do modelo em altas freqüências não é conhecida, é onde as incertezas podem ultrapassar o próprio ganho das plantas. As fontes de incertezas podem se agrupar em dois tipos principais. A seguir se descreve cada uma delas.

\subsubsection{Incertezas Estruturadas}

Nestes casos, a estrutura do modelo é completamente conhecida (inclusive em altas freqüências), mas alguns de seus parâmetros são incertos.

\subsubsection{Incertezas Sem-estrutura}

Perturbações dependentes da freqüência são exemplos típicos de incertezas sem- estrutura. Neste tipo de incertezas, geralmente não há interesse em determinar as origens das

\footnotetext{
${ }^{1}$ ver apendice $\mathrm{B}$
} 


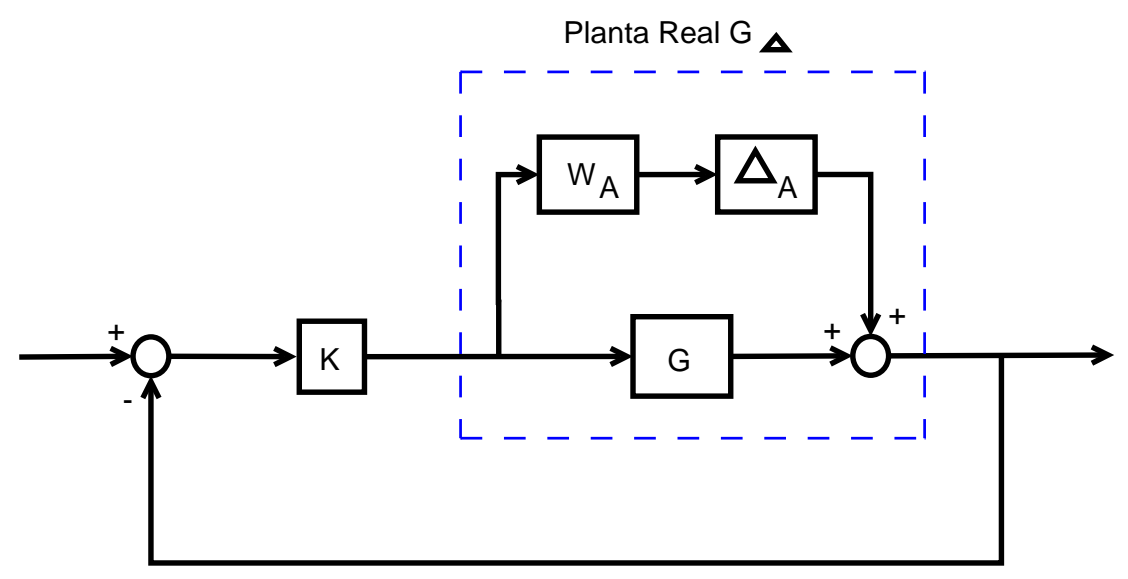

Figura 4.3: Incerteza aditiva.

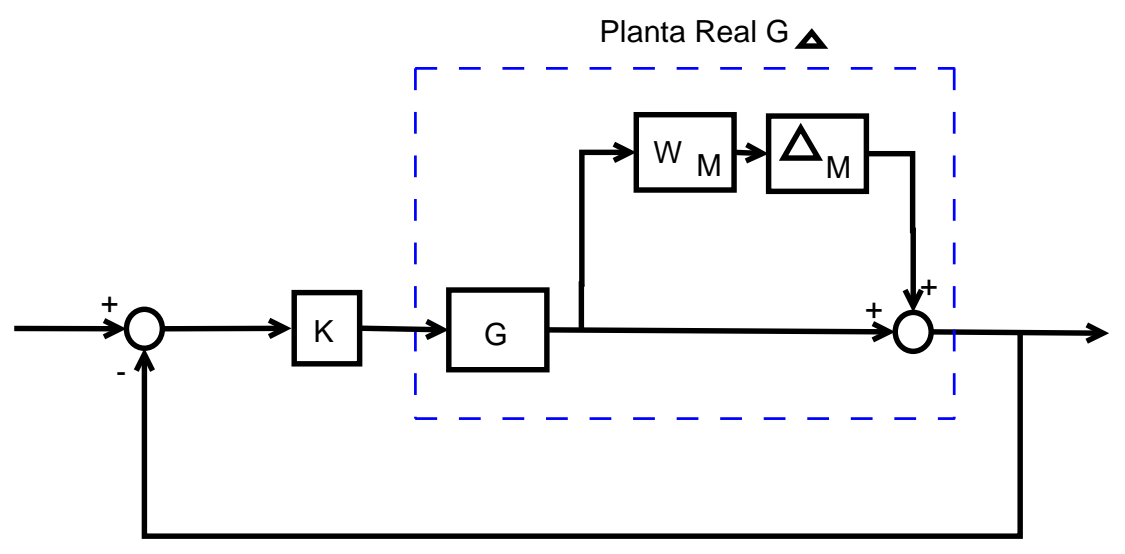

Figura 4.4: Incerteza multiplicativa na saída.

incertezas (procura-se apenas estabelecer seus limites superiores, por exemplo, através da norma $\mathcal{H}_{\infty}$ ). Embora desconhecidas, as incertezas mais comuns são:

- aditiva, modelada por:

$$
G_{\triangle}=G+\triangle_{A} W_{A}
$$

- multiplicativa na saída da planta, modelada por:

$$
G_{\triangle}=\left(I+\triangle_{M} W_{M}\right) G
$$

Existem outros tipos de incerteza, cujas ilustrações podem se encontrar em (SKOGESTAD; POSTLETHWAite, 1996). Nas expressões acima, $G_{\triangle}$, representa a planta real; $G$ a planta nominal; $\triangle_{A}$ é a incerteza aditiva; $\triangle_{M}$ é a incerteza multiplicativa; $W_{A}$ é a magnitude da incerteza aditiva; $W_{M}$ é a magnitude da incerteza multiplicativa. Para o pior caso 
possível $\left\|\Delta_{A}\right\|_{\infty} \equiv 1$ e $\left\|\Delta_{M}\right\|_{\infty} \equiv 1$.

A presença de incertezas no sistema é ilustrada nas figuras 4.3 e 4.4 .

\subsection{Especificações e Limitações de Desempenho}

Na teoria de controle avançado é usual a definição de diversas funções de transferência, ligadas à sensibilidade do sistema ${ }^{2}$.

Função de Sensibilidade $S$

$$
S(s)=(I+G K(s))^{-1}
$$

Função de Sensibilidade Complementar $T$

$$
T(s)=I-S(s)
$$

Função de Sensibilidade do Controlador $C$

$$
C(s)=K S(s)
$$

A forma das funções de sensibilidade tem um impacto direto sobre o desempenho e podem se sumarizados como abaixo (DONHA, 2003):

\section{Em baixas freqüências:}

1. $\bar{\sigma}(S)$ pequeno $\Rightarrow$ rejeição de distúrbios;

2. $\bar{\sigma}(T) \cong 1 \Rightarrow$ acompanhamento do sinal de referência.

\section{Em altas freqüências:}

1. $\bar{\sigma}(T)$ pequeno $\Rightarrow$ atenuação de ruídos;

2. $\bar{\sigma}(C)$ pequeno $\Rightarrow$ rejeição de ruídos;

3. $\bar{\sigma}(T)$ pequeno $\Rightarrow E R$ na presença de incerteza multiplicativa e

4. $\bar{\sigma}(C)$ pequeno $\Rightarrow E R$ na presença de incerteza aditiva.

Embora não se vá utilizar a formatação da função de transferência de malha $(L)$, como estratégia para sintetizar o controlador, apresentam-se também os requisitos para esta

\footnotetext{
${ }^{2}$ para sua demonstração veja-se (ZHOU; DOYLE; GLOVER, 1995; DONHA, 2003)
} 


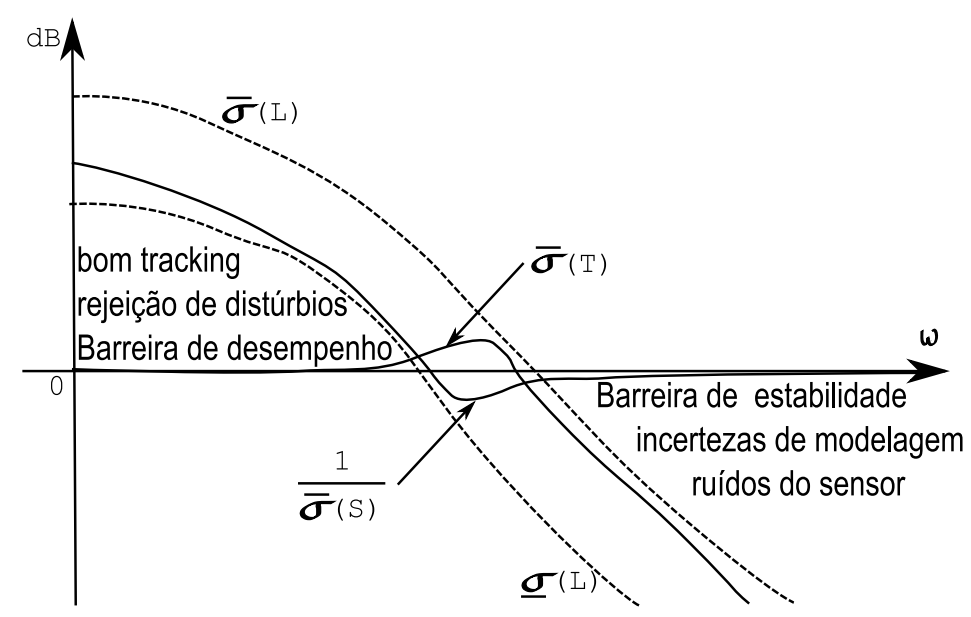

Figura 4.5: Barreiras de Robustez e Especificações para $S$ e $T$.

função, bastante úteis na hora de se analisar o projeto:

\section{Em baixas freqüências:}

1. $\underline{\sigma}(L)$ grande $\Rightarrow$ acompanhamento do sinal de referência;

2. $\underline{\sigma}(L)$ grande $\Rightarrow$ rejeição de distúrbios.

\section{Em altas freqüências:}

1. $\bar{\sigma}(L)$ pequeno $\Rightarrow$ atenuação de ruídos;

2. $\bar{\sigma}(K)$ pequeno $\Rightarrow$ rejeição de distúrbios;

3. $\bar{\sigma}(L)$ pequeno $\Rightarrow E R$ na presença de incerteza multiplicativa e

4. $\bar{\sigma}(K)$ pequeno $\Rightarrow E R$ na presença de incerteza aditiva.

A figura 4.5 ilustra os requisitos de forma para as funções de transferência de malha e para as funções de transferência de malha fechada. Nesta figura percebe-se que o pior caso em alta freqüência está associado com o $\bar{\sigma}(L)$, enquanto que o pior caso em baixa freqüência esta relacionado com o $\underline{\sigma}(L)$. É interessante observar-se também que para o acompanhamento do sinal de referência (tracking), para rejeição de perturbações e para insensibilidade paramétrica, geralmente, estabelecem-se restrições em baixa freqüência. A rejeição de ruídos provenientes dos sensores, minimização do consumo de energia de controle, e incertezas não estruturadas de modelagem impõem restrições em alta freqüência. 
É importante notar-se que na região de baixa freqüência, acima da linha $0 d B$ :

$$
\underline{\sigma}(L(j \omega)) \cong \frac{1}{\bar{\sigma}(S(j \omega))}
$$

e na região de alta freqüência:

$$
\bar{\sigma}(L(j \omega)) \cong \bar{\sigma}(T(j \omega))
$$

\subsubsection{Normalização}

A normalização é muito importante na análise e síntese de controladores avançados. Dada a natureza do problema, existem muitas variáveis a ser controladas, de magnitudes diferentes. Uma normalização adequada facilita a busca de parâmetros para a síntese do controlador.

O sistema descrito na equação (4.1) é normalizado através das matrizes de transformação $S_{u}, S_{x}$ e $S_{y}$, relacionadas com as variáveis de controle, estado, e saída, respectivamente:

$$
\begin{aligned}
& \widetilde{u} \leftarrow S_{u} u \\
& \widetilde{x} \leftarrow S_{x} x \\
& \widetilde{y} \leftarrow S_{y} y
\end{aligned}
$$

A normalização requer uma atitude crítica na hora de iniciar o projeto, no sentido de se avaliar o desempenho desejado e sua compatibilidade com os sinais presentes no sistema. Os requisitos são explicitados a partir das magnitudes máximas esperadas das perturbações, dos ruídos, das possíveis mudanças de referência e dos sinais de saída. As decisões serão baseadas nas magnitudes de sinais de referência e distúrbios, em outros sinais de entrada, como os sinais de controle e com os desvios nos sinais de saída (DONHA, 2003).

\subsection{Estabilidade}

A estabilidade de sistemas multivariáveis pode ser analisada através do critério de Nyquist, e requer considerações adicionais para o estudar a robustez da estabilidade, no caso MIMO. 


\subsubsection{Estabilidade Robusta}

Para ilustrar o problema de estabilidade robusta, consideremos uma planta com incerteza multiplicativa $\left\|\triangle_{M}(j \omega)\right\|_{\infty} \leq 1, \forall \omega$ na saída da planta, como ilustrado na figura 4.4. Admita-se por simplicidade que o sistema em malha fechada tenha estabilidade nominal, isto é, o sistema é estável quando $\triangle_{M}(j \omega)=0, \forall \omega$.

A família de funções de transferência de malha incertas é obtida a partir da função de transferência de malha nominal através da álgebra de diagrama de blocos, dada por:

$$
L_{\triangle}=G_{\triangle} K=\left(1+\triangle_{M} W_{M}\right) G K=L+\triangle_{M} W_{M} L, \quad\left\|\triangle_{M}(j \omega)\right\| \leq 1, \forall \omega
$$

De acordo com o critério de Nyquist, a estabilidade robusta estará garantida se nenhum elemento de $L_{\triangle}$ contornar o ponto -1 , ver figura 4.6.

A figura 4.6 apresenta o gráfico de Nyquist para um sistema escalar genérico, onde $\left\|W_{M} L\right\|$, é o raio de um disco ao redor de um membro nominal $L\left(j w_{i}\right)$, isto é, neste disco estão contidas todas as possíveis $L_{\triangle}$ para a freqüência $w_{i}$. A distância entre o ponto -1 e o $L\left(j w_{i}\right)$ é dada por $\left|1+L\left(j w_{i}\right)\right|$. Considere-se ainda que toda a família de funções de transferência de malha $L_{\triangle}(j w)$ seja estável. Da figura 4.6, o ponto -1 não será envolvido mesmo ocorrendo incerteza da pior espécie $\left(\triangle_{M}=1\right)$ quando:

$$
\begin{aligned}
\left|W_{M} L\right| & <|1+L|, \forall \omega \\
& \Longleftrightarrow\left|W_{M} \frac{L}{1+L}\right|<1, \forall \omega \\
& \Longleftrightarrow\left|W_{M} T\right|<1, \forall \omega \\
& \Longleftrightarrow\left\|W_{M} T\right\|_{\infty}<1 \\
& \Longleftrightarrow\|T\|_{\infty}<\left\|W_{M}\right\|_{\infty}^{-1}
\end{aligned}
$$

A equação (4.26) sugere que deve existir um limite superior para $T$ (sensibilidade complementar) para se obter estabilidade robusta .

- caso escalar

$$
E R \Longleftrightarrow|T|<\left|\frac{1}{W_{M}}\right|
$$

- caso multivariável

$$
\begin{aligned}
E R & \Longleftarrow \bar{\sigma}\left(W_{M} T\right)<1, \forall \omega \\
& \Longleftarrow \bar{\sigma}(T)<\frac{1}{\bar{\sigma}\left(W_{M}\right)}, \forall \omega
\end{aligned}
$$




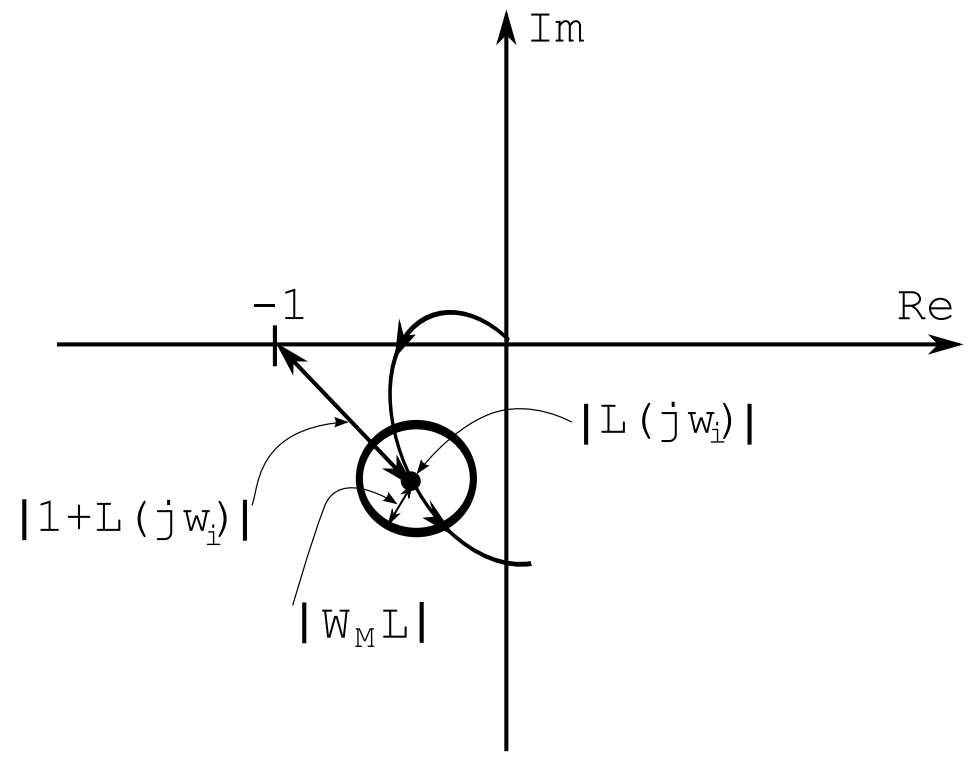

Figura 4.6: Estabilidade Robusta, SISO.

a condição acima é suficiente, mas não necessária, isto é, uma violação desta condição não implica necessariamente a perda da estabilidade robusta, mas se ela ocorre a $E R$ está garantida (SKOGESTAD; POSTLETHWAITE, 1996).

A expressão (4.28) conduz à seguinte expressão para o caso multivariável:

$$
\bar{\sigma}(L)<\frac{1}{\bar{\sigma}\left(W_{M}\right)} \text {, para } \omega \text { em altas freqüências }
$$

Uma prática muito comum é agregarem-se todos os efeitos de incertezas da planta em um único bloco multiplicativo fictício, de modo que seja possível trabalhar-se com a expressão (4.28).

Em geral, tem-se que:

$$
\begin{aligned}
& \|S\|_{\infty},\|T\|_{\infty}, \text { grandes } \Rightarrow E R \text { pobre } \\
& \|S\|_{\infty},\|T\|_{\infty}, \text { pequenas } \nRightarrow \text { necessariamente } E R
\end{aligned}
$$

\subsection{Desempenho}

Embora a estabilidade da malha fechada seja o objetivo prioritário, outro objetivo de igual relevância é a capacidade do sistema manter o desempenho aceitável. Tipicamente, todo sistema está sujeito a perturbações externas, como por exemplo: ruídos nos sensores (navegação e posicionamento), rajadas de vento, ondas e correntezas. Estas perturbações determinam erros de regulação e de acompanhamento do sinal de referência, que tendem a crescer com a severidade das perturbações na maioria de casos práticos. O sistema 
em malha fechada acaba operando com um desempenho considerado inaceitável. Daí a necessidade de utilizar um teste de desempenho (DONHA, 2003). O objetivo deste teste de desempenho é indicar a pior degradação de desempenho associada a um tipo de perturbação (ZHOU; DOYLE; GLOVER, 1995).

A amplitude máxima das funções de sensibilidade dão bons indicativos de desempenho. Segundo regras práticas, um bom desempenho, tipicamente apresenta:

$$
\|S\|_{\infty}<6 d B, \quad\|T\|_{\infty}<2 d B
$$

Em termos gerais, considera-se que um sistema controlado tem bom desempenho nas seguintes situações:

- quando ele é capaz de seguir um sinal de referência com bastante proximidade;

- quando sua capacidade de recuperação frente a distúrbios externos é boa;

- quando ele apresenta boas características de resposta, apesar das eventuais incertezas de modelagem;

- quando ele apresenta boa rejeição a ruídos de medida na saída.

\subsubsection{Desempenho Robusto}

Parte-se da definição de DR para o caso escalar:

$$
\begin{array}{ccc}
\text { DR } \quad \operatorname{def}_{\Leftrightarrow} \quad\left|W_{s} S\right|<1 \quad \forall S, \forall \omega \\
\Leftrightarrow \quad\left|W_{s}\right|<\left|1+L_{\Delta}\right| \quad \forall L_{\Delta}, \forall \omega
\end{array}
$$

onde $W_{s}$ é a função de ponderação do sinal do erro ${ }^{3}, S$ é a função de sensibilidade do sistema.

A derivação da condição de desempenho para sistemas escalares pode ser feita de modo gráfico, como ilustra a figura 4.7.

Para o DR, requer-se que nenhum membro da família de funções incertas $L_{\Delta}$ cruze o disco de raio $W_{s}$ com centro em -1. Portanto, se deseja que:

$$
\left|W_{s}\right|+\left|W_{M} L\right|<|1+L|, \quad \forall \omega
$$

E multiplicando ambos os lados da inequação por $|S|$, obtém-se:

\footnotetext{
${ }^{3}$ função de ponderação da sensibilidade do sistema
} 


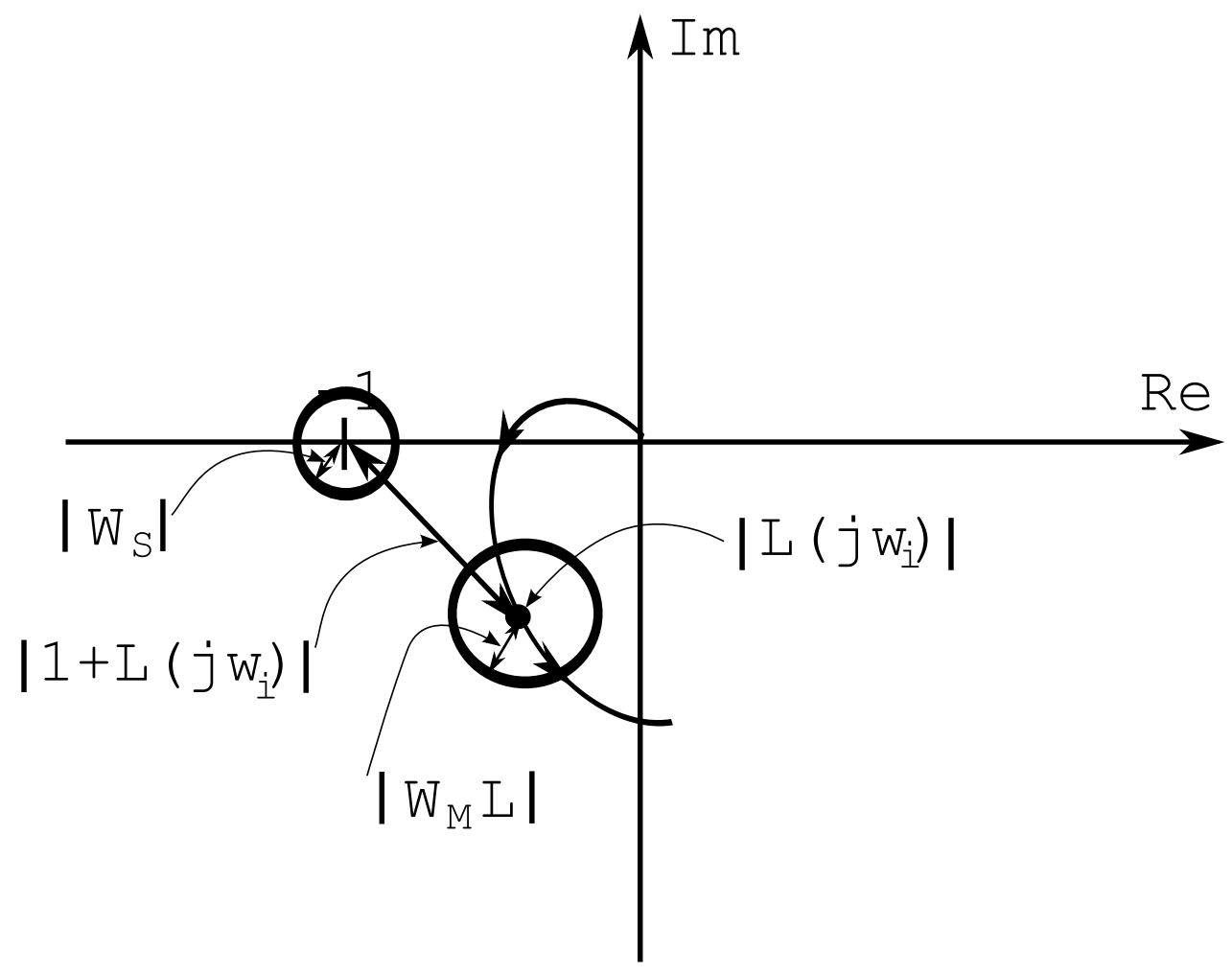

Figura 4.7: Desempenho Robusto .

$$
\begin{gathered}
\left|W_{s} S\right|+\left|W_{M} L S\right|<1, \quad \forall \omega \\
\Leftrightarrow\left|W_{s} S\right|+\left|W_{M} T\right|<1, \quad \forall \omega \\
\Leftrightarrow \quad \max _{\omega}\left(\left|W_{s} S\right|+\left|W_{M} T\right|\right)<1
\end{gathered}
$$

A equação (4.37) é a condição de Desempenho Robusto em termos das funções de malha fechada.

A seguir, apresenta-se a generalização de Desempenho Robusto $(D R)$, para o caso multivariável, com perturbação na saída, conforme o exposto em (ZHOU; DOYLE; GLOVER, 1995).

Considere-se a figura 4.4 para o tipo de incertezas multiplicativas na saída, onde $W_{M}$ é a função de ponderação que dá informação das incertezas $\triangle_{M}, W_{S}$ é a função de ponderação do sinal de erro, $S$ é a função de sensibilidade e $T$ é a função de sensibilidade complementar. Para sistemas MIMO, no pior caso possível $\left(\|\triangle\|_{\infty}=1\right)$, tem-se:

$$
\bar{\sigma}\left(W_{S} S\right)+\bar{\sigma}\left(W_{M} T\right) \leq 1, \forall \omega
$$

A equação (4.38) para sistemas multivariáveis é a generalização da expressão (4.37) para sistemas escalares. 


\subsubsection{Formatação das Funções de Sensibilidade}

A seguir, apresentam-se métodos práticos para a formatação das funções de Sensibilidade. A aplicação em sistemas SISO é clara, porém em sistemas MIMO é de dificuldade considerável.

De acordo com a figura 4.8, a formatação da função de sensibilidade $S$ é feita a partir do conhecimento de especificações de desempenho, procurando-se atingir

$$
\begin{aligned}
& |S(s)| \leq\left|\frac{s}{s / M_{s}+\omega_{b}}\right|, s=j \omega, \forall \omega \\
& \Longleftrightarrow\left|W_{s} S\right| \leq 1, W_{s}=\frac{s / M_{s}+\omega_{b}}{s} .
\end{aligned}
$$

Para uma realização prática:

$$
W_{s}=\frac{s / M_{s}+\omega_{b}}{s+\omega_{b} \varepsilon}
$$

onde $M_{s}$ limita o sobresinal, normalmente em $2 d B$ de amplitude; $w_{b}$ é limitada pela largura de banda do sistema; $\varepsilon$ facilita a implementação computacional do filtro $W_{s}$.

De acordo com a figura 4.9, a formatação da função de sensibilidade de custo do controlador $C$ é feita da mesma maneira que o caso anterior, porém com a seguinte função de ponderação:

$$
W_{C}=\frac{s+w_{b c} / M_{u}}{\varepsilon_{1} s+w_{b c}}
$$

De modo similar, para a função de sensibilidade complementar $T$, usa-se a seguinte função de ponderação:

$$
W_{T}=\frac{s+w_{b t} / M_{t}}{\varepsilon_{2} s+w_{b t}}
$$

Em todas as ponderações anteriores foi admitido que o canal filtrado (ponderado) é sempre escalar; para o caso MIMO pode-se também utilizar uma formatação por canal (sinal), mas a busca de parâmetros torna-se mais intrincada. 


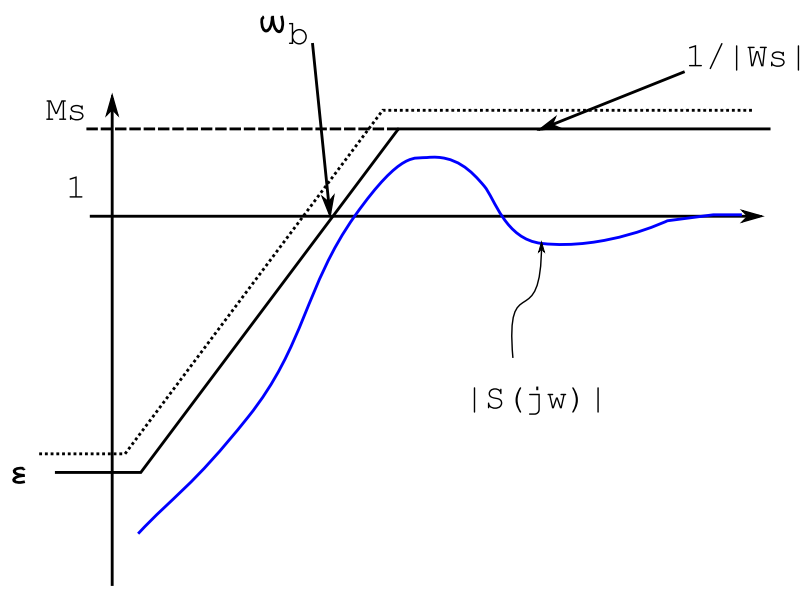

Figura 4.8: Função de Ponderação $W_{s}$.

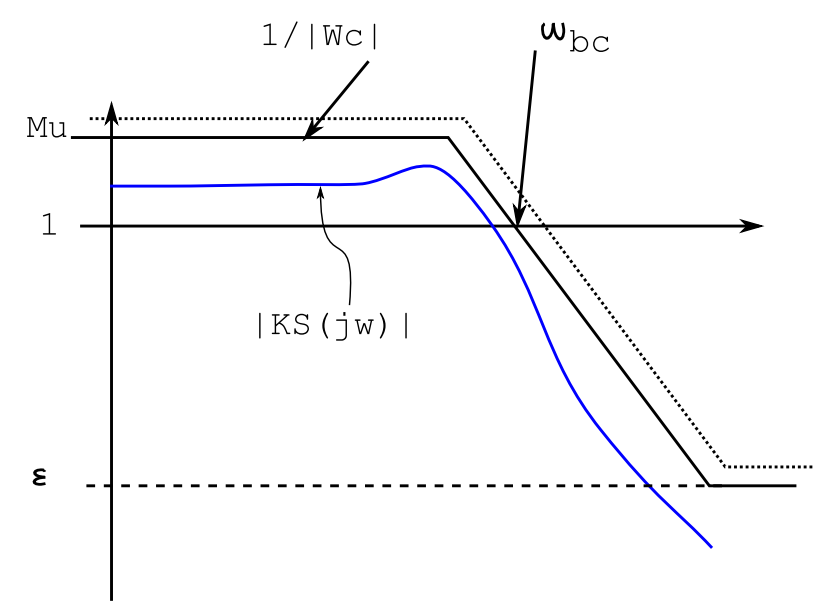

Figura 4.9: Função de Ponderação $W_{u}$. 
Planta Generalizada

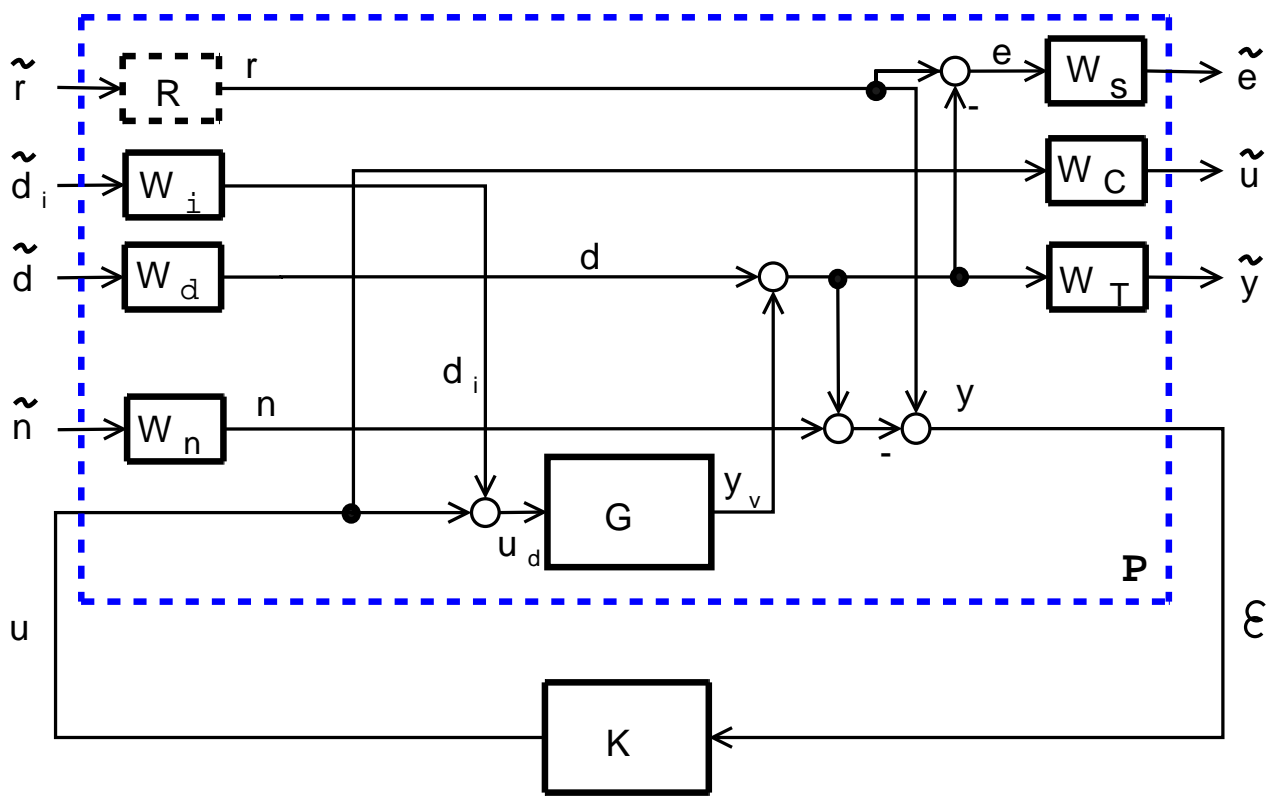

Figura 4.10: Configuração Genérica de Dois Portos.

\subsubsection{Configuração de Dois Portos}

A estrutura clássica é modificada para o emprego de técnicas de controle avançado. A nova configuração é conhecida como Configuração de Dois Portos. Assim, o sistema após manipulado adquire a configuração da figura 4.10, onde:

- $w=\left[\begin{array}{llll}\widetilde{r} & \widetilde{d}_{i} & \widetilde{d} & \widetilde{n}\end{array}\right]^{T}$ : sinais exógenos

- $z=\left[\begin{array}{lll}\widetilde{e} & \widetilde{u} & \widetilde{y}\end{array}\right]^{T}$ : sinal de saída controlado

- P: Planta generalizada, que tem cinco entradas ( $w$ e $u$ ), e quatro saídas $(z$ e $\varepsilon$ ).

Portanto, a planta generalizada (estendida) $P(s)$ é composta da planta nominal $G(s)$ e das funções de ponderação $W_{\bullet}(s)$ e $R(s)$, como se mostra na figura 4.10. O sistema também pode ser expresso na forma matricial por:

$$
\begin{aligned}
{\left[\begin{array}{l}
z \\
\varepsilon
\end{array}\right] } & =P(s)\left[\begin{array}{l}
w \\
u
\end{array}\right]=\left[\begin{array}{ll}
P_{11}(s) & P_{12}(s) \\
P_{21}(s) & P_{22}(s)
\end{array}\right]\left[\begin{array}{l}
w \\
u
\end{array}\right] \\
u & =K(s) \varepsilon
\end{aligned}
$$


onde a realização em espaço de estados de $P(s)$ é como segue:

$$
P(s)=\left[\begin{array}{c|cc}
A & B_{1} & B_{2} \\
\hline C_{1} & D_{11} & D_{12} \\
C_{2} & D_{21} & D_{22}
\end{array}\right]
$$

\subsection{Metodologia da Sensibilidade Mista}

Sensibilidade Mista é o nome dado ao problema de formatação da função de sensibilidade $S=(I+G K)^{-1}$, da função de sensibilidade complementar $T=I-S$ e da função de sensibilidade do controlador $C=K S$.

A partir da figura 4.10, observa-se que $S$ é a função de transferência entre o erro $e$ e o distúrbio externo $d$, com isto, $1 / W_{S}$ terá uma forma parecida a um filtro passa-alta, e $1 / W_{C}$ e $1 / W_{T}$ terão formas parecidas a filtros passa-baixas (ver equações $(4.40),(4.41)$ e (4.42)).

Dado que o distúrbio ocorre tipicamente em baixa freqüência, este será minimizado, se o valor singular máximo de $S$ é pequeno em baixas freqüência. O objetivo, então, é minimizar $\left\|W_{S} S\right\|_{\infty}$. Em termos práticos, também procura-se bom acompanhamento do sinal de referência, isto se consegue com a construção da seguinte pilha:

$$
N=\left\|\left[\begin{array}{l}
W_{S} S \\
W_{T} T
\end{array}\right]\right\|_{\infty}
$$

Caso se deseje estabilidade robusta (ER) além de desempenho robusto (DR), objetivos que podem ser traduzidos em pequena sensibilidade a ruídos e incertezas de qualquer espécie, e adicionalmente se requeira ainda a penalização do uso excessivo de energia para controle, pode ser escolhida a seguinte pilha.

$$
N=\left\|\left[\begin{array}{l}
W_{S} S \\
W_{C} C \\
W_{T} T
\end{array}\right]\right\|_{\infty}
$$

\subsection{Controlador}

A solução do problema de controle usando a norma $\mathcal{H}_{\infty}$ é exposta sucitamente nesta seção. 


\subsubsection{Formulação do Problema de Controle}

O problema do controle $\mathcal{H}_{\infty}$ pode ser formulado da seguinte maneira: "determinar um controlador $K(s)$ que estabilize o sistema e tal que a matriz de transferência $N$ entre $\omega$ e $z$, seja limitada superiormente pela norma $\mathcal{H}_{\infty}$ ".

Considere-se a configuração de dois portos da figura 4.10. Assim, a equação abaixo é a expressão formal do problema:

$$
\|N\|_{\infty} \leq \gamma
$$

Novamente, considere-se a expressão (4.46), pela abordagem de sensibilidade mista:

$$
\|N\|_{\infty}=\left\|\left[\begin{array}{l}
W_{S} S \\
W_{C} C \\
W_{T} T
\end{array}\right]\right\|_{\infty} \leq \gamma
$$

O problema de controle se traduz em: determinar o controle $K(s)$ de forma a minimizar a norma infinita da matriz de transferência $N$ entre o sinal exógeno $w$ e o vetor de saídas controladas $z$.

As seguintes hipóteses são consideradas nos problemas $\mathcal{H}_{\infty}$.

1. $\left(A, B_{2}, C_{2}\right)$ é estabilizável e detectável;

2. $D_{12}$ e $D_{21}$ têm (posto) completo;

3. $\left[\begin{array}{cc}A-j \omega I & B_{2} \\ C_{1} & D_{12}\end{array}\right]$ tem posto de coluna completo para todo $\omega$;

4. $\left[\begin{array}{cc}A-j \omega I & B_{1} \\ C_{2} & D_{21}\end{array}\right]$ tem posto de linha completo para todo $\omega ;$

5. $D_{11}=0, D_{22}=0$;

6. $D_{12}=\left[\begin{array}{l}0 \\ I\end{array}\right]$ e $D_{21}=\left[\begin{array}{ll}0 & I\end{array}\right]$

7. $D_{12}^{T} C_{1}=0$ e $B_{1} D_{21}^{T}=0$ e

8. $\left(A, B_{1}\right)$ é estabilizável e $\left(A, C_{1}\right)$ é detectável.

A hipótese (1) garante a existência de que $K$ que estabilize o sistema, e (2) é condição suficiente para que os controladores sejam próprios e realizáveis. Os items (3) e (4) garantem a não anulação de pólos e zeros no eixo imaginário, o que implicaria instabilidade 
da malha fechada. (5) é hipótese convencional em $\mathcal{H}_{2}$ e em alguns casos de $\mathcal{H}_{\infty}$ e será adotada por simplicidade. $D_{11}=0$ implica que não há alimentação direta entre $w$ e $z$, de tal forma que a função de transferência entre estas variáveis é estritamente própria; $D_{22}=0$ implica que não há alimentação direta entre $u$ e $y$, de tal forma que a função de transferência entre estas variáveis é também estritamente própria. Esta hipótese é desnecessária em controle $\mathcal{H}_{\infty}$. As igualdades do item (6) geralmente são conseguidas através da normalização dos sinais $u$ e $y$, e uma transformação de unitária de $w$ e $z$ e não tiram a generalidade do problema. (7) é comum em $\mathcal{H}_{2}$. Por último, (8) pode substituir (3) e (4) se (7) é verdadeiro.

Em geral, os algoritmos $\mathcal{H}_{\infty}$ encontram um controlador sub-ótimo, tal que para um valor $\gamma$, encontra-se um controlador $K$ que estabilize a matriz de transferência na norma- $\infty$ $\left(\|N\|_{\infty}<\gamma\right)$. Encontrar um controlador $\mathcal{H}_{\infty}$ ótimo é complicado numericamente e teoricamente. Isto contrasta com a teoria $\mathcal{H}_{2}$ onde o controlador ótimo é único e pode ser encontrado através das equações de Ricatti.

\subsubsection{Algoritmo geral $\mathcal{H}_{\infty}$}

Para a configuração genérica de dois portos (figura 4.10), e a realização (4.7), considerando as 8 hipóteses mencionadas na página (49), existe um $K(s)$ que estabilize o sistema, de maneira que $\|N\|_{\infty}<\gamma$, se e apenas se:

i) $X_{\infty}$ é solução da equação de Ricatti

$$
A^{T} X_{\infty}+X_{\infty} A+C_{1}^{T} C_{1}+X_{\infty}\left(\gamma^{-2} B_{1} B_{1}^{T}-B_{2} B_{2}^{T}\right) X_{\infty}=0
$$

tal que $\operatorname{Re} \lambda_{i}\left[A+\left(\gamma^{-2} B_{1} B_{1}^{T}-B_{2} B_{2}^{T}\right) X_{\infty}\right]<0, \forall i$ e

ii) $Y_{\infty} \geq 0$ é solução da equação de Ricatti

$$
A Y_{\infty}+Y_{\infty} A^{T}+B_{1} B_{1}^{T}+Y_{\infty}\left(\gamma^{-2} C_{1}^{T} C_{1}-C_{2}^{T} C_{2}\right) Y_{\infty}=0
$$

tal que $\operatorname{Re} \lambda_{i}\left[A+Y_{\infty}\left(\gamma^{-2} C_{1}^{T} C_{1}-C_{2}^{T} C_{2}\right)\right]<0, \forall i$; e

iii) $\rho\left(X_{\infty} Y_{\infty}\right)<\gamma^{2}$

Todos os controladores são determinados pela transformação ${ }^{4} F_{l}$ :

$$
K=F_{l}\left(K_{c}, Q\right)
$$

\footnotetext{
${ }^{4} F_{l}$ transformação LFT, veja-se apêndice (B.3)
} 
onde:

$$
\begin{gathered}
K_{c}(s)=\left[\begin{array}{c|cc}
A_{\infty} & -Z_{\infty} L_{\infty} & Z_{\infty} B_{2} \\
\hline F_{\infty} & 0 & I \\
-C_{2} & I & 0
\end{array}\right] \\
F_{\infty}=-B_{2}^{T} X_{\infty}, \quad L_{\infty}=-Y_{\infty} C_{2}^{T}, \quad Z_{\infty}=\left(I-\gamma^{-2} Y_{\infty} X_{\infty}\right)^{-1}, \\
A_{\infty}=A+\gamma^{-2} B_{1} B_{1}^{T} X_{\infty}+B_{2} F_{\infty}+Z_{\infty} L_{\infty} C_{2},
\end{gathered}
$$

$\rho$ é o raio espectral, correspondente ao maior autovalor do produto das soluções matriciais das equações de Ricatti; $Q(s)$ é uma função de transferência própria e estável, tal que $\|Q\|_{\infty}<\gamma$.

Para $Q(s)=0$, chega-se a:

$$
K(s)=K_{c_{11}}(s)=-F_{\infty}\left(S I-A_{\infty}\right)^{-1} F_{\infty}
$$

O controlador da expressão (4.55) é chamado de controlador central e tem o mesmo número de estados que a planta estendida $P(s)$. Este controlador utiliza também o Princípio de Separação como fica explícito na seguinte expressão (DONHA, 2003):

$$
\begin{gathered}
\dot{\hat{x}}=A \hat{x}+B_{1} \gamma^{-2} B_{1}^{T} X_{\infty} \hat{x}+B_{2} u+Z_{\infty} L_{\infty}\left(C_{2} \hat{x}-y\right) \\
u=F_{\infty} \hat{x}
\end{gathered}
$$




\section{$5 \quad$ Resultados}

\subsection{Introdução}

Neste capítulo apresentam-se os resultados da aplicação das teorias delineadas no decorrer dos capítulos três e quatro.

O método de Sensibilidade Mista é utilizado para formatar as funções de sensibilidade $S$, sensibilidade complementar $T$ e sensibilidade do controlador $C$.

\subsection{Apresentação do Problema}

O sistema VSA é composto dos seguintes subsistemas:

1. subsistema de sensores: composto por equipamentos acústicos, mecânicos e/ou elétricos, projetados para medir $\phi, \theta, \dot{\psi}$ e $\dot{z}$;

2. subsistema de atuação: propulsores, lemes horizontais e verticais, que são encarregados de produzir forças e momentos para manter a posição e atitude desejadas e

3. subsistema lógico: que processa a informação recebida dos sensores para comandar o sistema atuador. A principal tarefa deste subsistema é operar como controlador do sistema.

\subsection{Modelo Não-linear}

O modelo não linear do VSA foi desenvolvido no capítulo 3. A seguir apresentam-se resultados da sua solução numérica no domínio do tempo.

Consideram-se inicialmente quatro manobras principais com o fim de validar o modelo VSA não linear. Os resultados mostram a ação dos atuadores sobre o veículo, ainda não 
controlado.

Todas as simulações foram feitas utilizando o algoritmo Runge Kutta de 4a Ordem, com duração de 100 segundos e intervalo de integração de 0,01 segundo.

- Caso 1, simulação com condição inicial: velocidade de avanço de $2 \mathrm{~m} / \mathrm{s}$; leme vertical a $15^{\circ}$ e leme horizontal a $0^{\circ}$ (figura 5.1).

- Caso 2, simulação com condição inicial: velocidade de avanço de $2 \mathrm{~m} / \mathrm{s}$; leme vertical a $-15^{\circ}$ e leme horizontal a $0^{\circ}$ (figura 5.2).

- Caso 3, simulação com condição inicial; velocidade de avanço de $2 \mathrm{~m} / \mathrm{s}$; leme vertical a $0^{\circ}$ e leme horizontal a $15^{\circ}$ (figura 5.3).

- Caso 4, simulação com condição inicial: velocidade de avanço de $2 \mathrm{~m} / \mathrm{s}$; leme vertical a $0^{\circ}$ e leme horizontal a $-15^{\circ}$ (figura 5.4).

Nos casos 1 e 2, a ação do leme vertical leva o veículo para a lateral na direção de bombordo e boreste, respectivamente ${ }^{1}$. A posição de deriva $(y)$ decresce ou cresce dependendo da posição do leme vertical (figuras 5.1(b) e 5.2(b)). O ângulo de guinada, da mesma forma, decresce ou cresce (figuras 5.1(d) e 5.2(d)).

Nos casos 3 e 4, a ação do leme horizontal leva o veículo a emergir ou submergir, respectivamente. Portanto, a posição de arfagem $(z)$ decresce ou cresce dependendo da posição do leme horizontal (figuras 5.3(b) e 5.4(b)). O ângulo de caturro, ao contrario de $z$, cresce ou decresce (figuras $5.3(\mathrm{~d})$ e $5.4(\mathrm{~d})$ ).

Pode-se observar, nos quatro casos, que o arrasto hidrodinâmico é notável para o avanço. O movimento de balanço apresenta característica oscilatória muito pouca amortecida, com uma freqüência aproximada de $0,94 \mathrm{rad} / \mathrm{s}(0,15 \mathrm{~Hz})$. Já o caturro, apesar de ser oscilatório, é fortemente amortecido, ver figuras 5.1(c) e 5.2(c). Finalmente, todas as respostas apresentam um forte acoplamento entre seus movimentos, na lateral e na vertical. De fato, o VSA ainda não foi controlado.

\footnotetext{
${ }^{1}$ As figuras 2.4 e 3.1 ilustram os sentidos positivo e negativo para as rotações do veículo e dos lemes.
} 


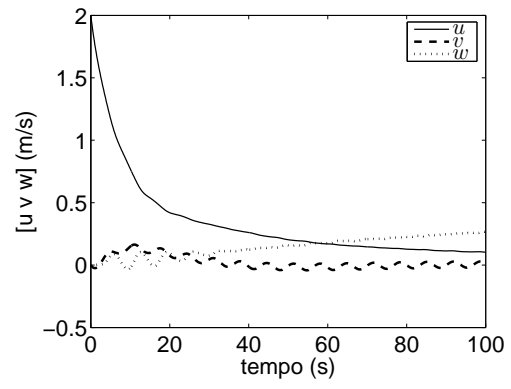

(a) velocidade linear

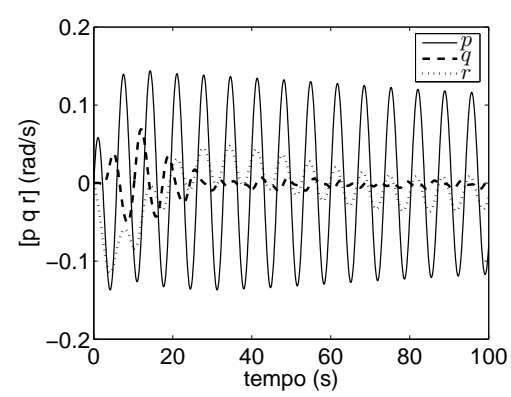

(c) velocidade rotacional

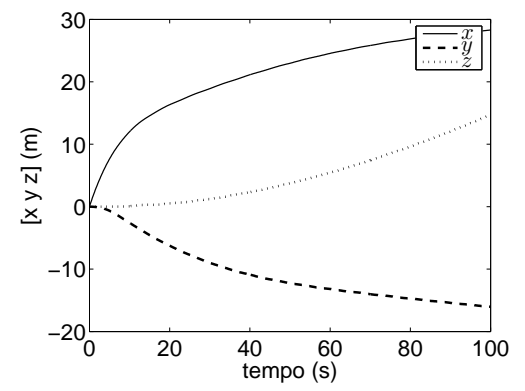

(b) posição

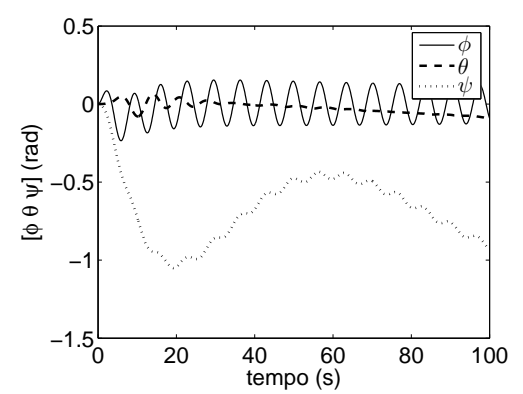

(d) atitude

Figura 5.1: Modelo não-linear sem controle: $\delta_{r}=15^{\circ}$ e $\delta_{s}=0^{\circ}$

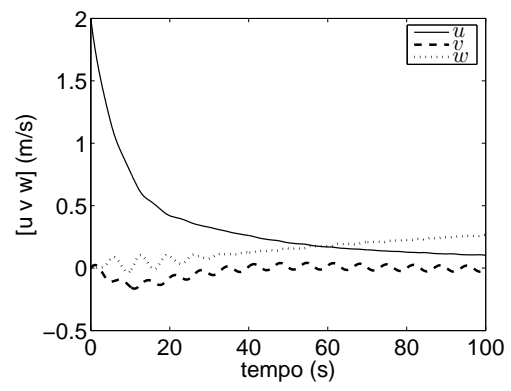

(a) velocidade linear

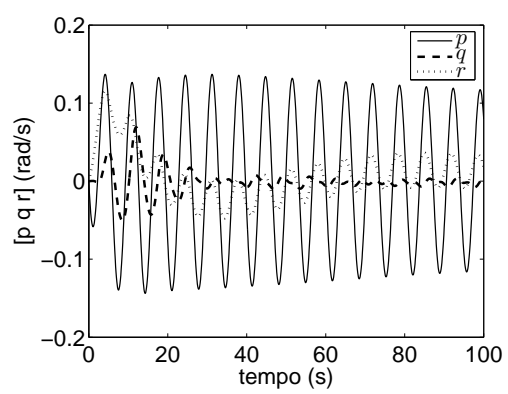

(c) velocidade rotacional

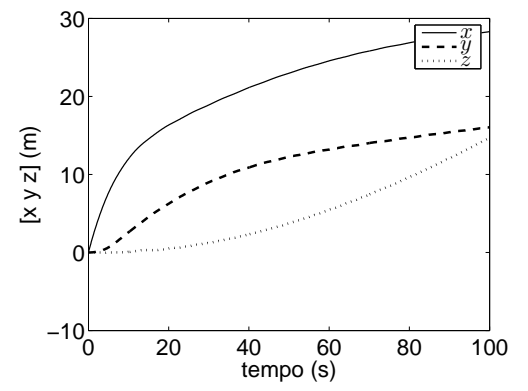

(b) posição

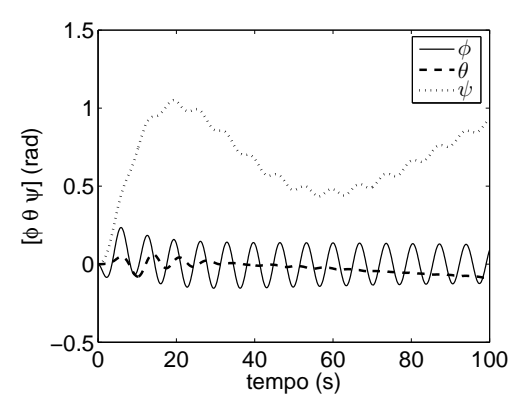

(d) atitude

Figura 5.2: Modelo não-linear sem controle: $\delta_{r}=-15^{\circ}$ e $\delta_{s}=0^{\circ}$ 


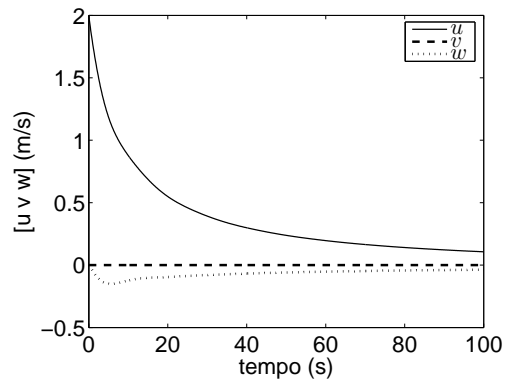

(a) velocidade linear

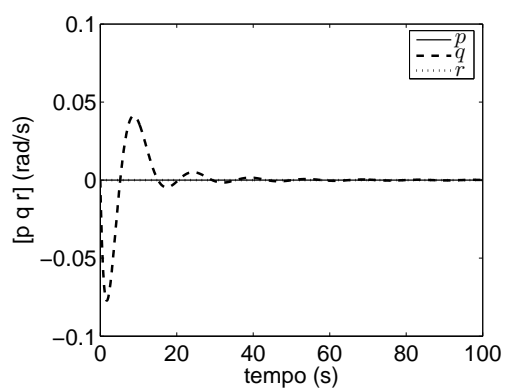

(c) velocidade rotacional

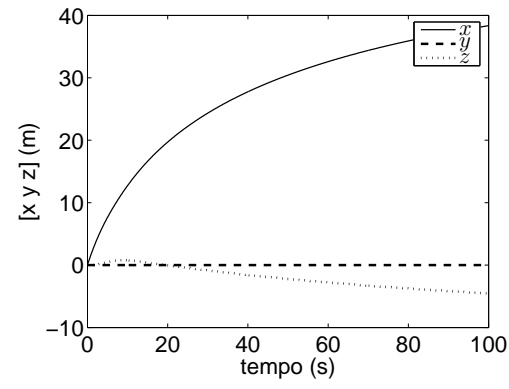

(b) posição

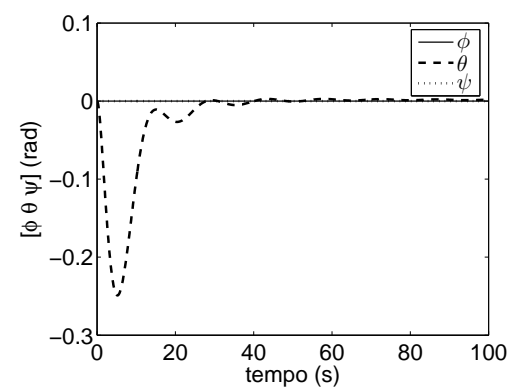

(d) atitude

Figura 5.3: Modelo não-linear sem controle: $\delta_{r}=0^{\circ}$ e $\delta_{s}=15^{\circ}$

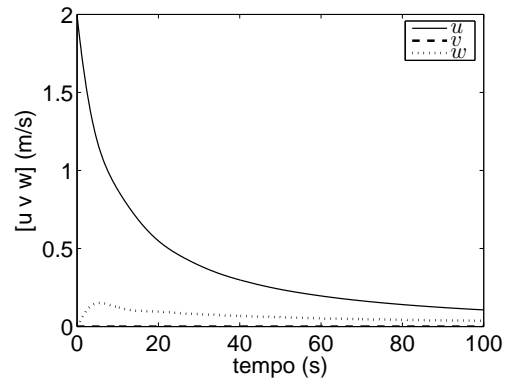

(a) velocidade linear

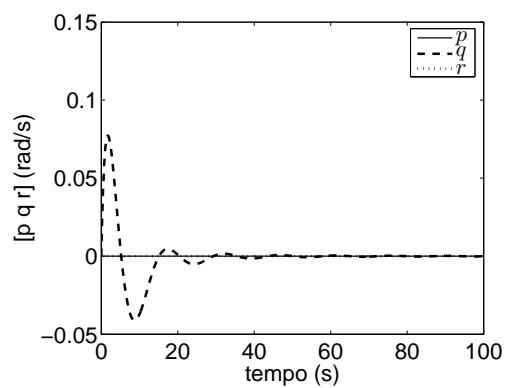

(c) velocidade rotacional

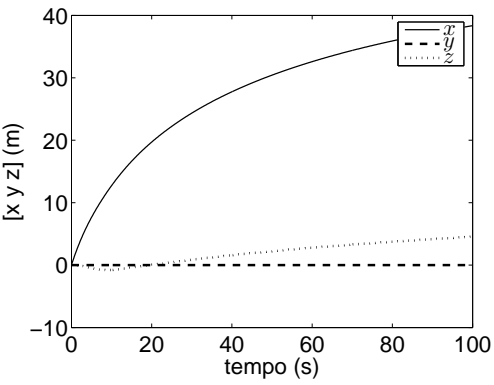

(b) posição

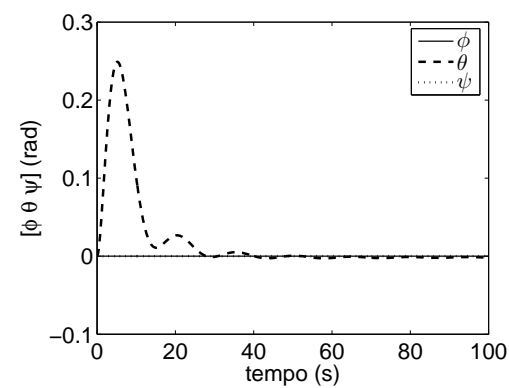

(d) atitude

Figura 5.4: Modelo não-linear sem controle: $\delta_{r}=0^{\circ}$ e $\delta_{s}=-15^{\circ}$ 


\subsection{Modelo Linear}

O ponto de operação típica do submersível é sua velocidade de cruzeiro $(u=2 \mathrm{~m} / \mathrm{s})$. A seção seguinte apresenta a simulação do modelo VSA linearizado ao redor desta velocidade. A equação (4.1) apresenta o sistema linear na forma clássica. As matrizes do sistema são: $A$, a matriz de estados de dimensão $(12 \times 12) ; B$, a matriz de controle, de dimensão $(2 \times 2)$; $C$, a matriz do observador, de dimensão $(4 \times 12)$ e $D$ a matriz de alimentação direta, de dimensão $(4 \times 4)$.

Alguns estados $(x, y, z$ e $\psi$ ) não são considerados, pois são desacoplados das outras variáveis, podendo ser controlados e avaliados independentemente. Assim as matrizes na equação (4.1) ficam reduzidas à dimensão: $A(8 \times 8), B(8 \times 2), C 4 \times 8)$ e finalmente, $D$ de $(4 \times 2)$. Seus valores numéricos estão no anexo A.2.

apresenta-se agora as variáveis do sistema linear invariante no tempo (LIT):

vetor de estado:

$$
x=\left[\begin{array}{ccc}
u & - & \mathrm{m} / \mathrm{s} \\
v & - & \mathrm{m} / \mathrm{s} \\
w & - & \mathrm{m} / \mathrm{s} \\
p & - & \mathrm{rad} / \mathrm{s} \\
q & - & \mathrm{rad} / \mathrm{s} \\
r & - & \mathrm{rad} / \mathrm{s} \\
\phi & - & \mathrm{rad} \\
\theta & - & \mathrm{rad}
\end{array}\right]
$$

vetor de controle (entradas):

$$
u=\left[\begin{array}{lll}
\delta_{r} & - & \mathrm{rad} \\
\delta_{s} & - & \mathrm{rad}
\end{array}\right]
$$

e finalmente, vetor de saídas:

$$
y=\left[\begin{array}{ccc}
\phi & - & \mathrm{rad} \\
\theta & - & \mathrm{rad} \\
\dot{\psi} & - & \mathrm{rad} / \mathrm{s} \\
\dot{z} & - & \mathrm{m} / \mathrm{s}
\end{array}\right]
$$

Para a linearização, admite-se que a força de arrasto seja idêntica à força de empuxo dos propulsores, de modo que o VSA opere com velocidade constante:

$$
T_{p}=\frac{R_{v}}{1-c_{t}}
$$

A figura 5.5 ilustra o núcleo de variáveis acopladas do modelo a ser utilizado no procedimento de controle robusto, que é analisado a seguir. 


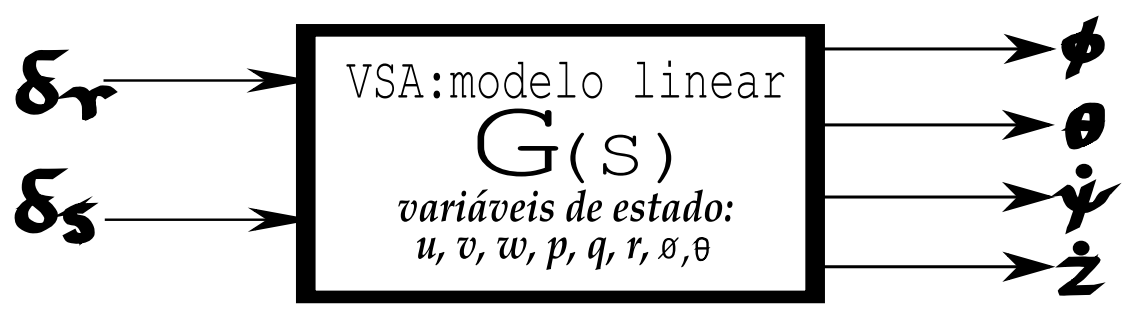

Figura 5.5: Modelo Linear Invariante no Tempo do Veículo Submersível Autônomo, .

\subsubsection{Análise do Modelo Linear do VSA}

Os pólos do sistema não controlado de dimensão 8 são dados na tabela 5.1 e ilustrados na figura 5.6. Conclui-se, então, que o sistema linear construído para o VSA é instável. Obviamente, a mesma análise pode ser feita através dos autovalores, cujos autovetores associados aparecem na tabela 5.2.

Tabela 5.1: Pólos do Sistema Linear Invariante no Tempo

\begin{tabular}{|l|r|r|}
\hline Descrição & Pólos & Freqüência (rad/s) \\
\hline \hline arfagem/caturro (oscilação) & $-0,429 \pm 0,311 i$ & 0,53 \\
caturro & $-0,141$ & 0,141 \\
balanço (oscilação) & $0,0306 \pm 0,918 i$ & 0,918 \\
deriva/balanço (oscilação) & $-0,119 \pm 0,322 i$ & 0,343 \\
velocidade de avanço & $-0,324$ & 0,324 \\
\hline
\end{tabular}

Na figura 5.7 são ilustradas as magnitudes dos autovetores do modelo LIT. Pode-se observar o forte acoplamento entre os movimentos de arfagem/caturro (figura 5.7(a)), deriva/balanço (figura 5.7(d)). O acoplamento deriva/balanço (variáveis $\phi$ e $\psi$ ) é freqüente em veículos marítimos, por isso sistemas mais complexos usam técnicas de estabilização de balanço (roll stabilization) para contrapor este efeito, (FOSSEN, 1994).

O movimento de balanço também é oscilatório (figura 5.7(c)) devido à forma do casco e aos esforços restauradores. A possível causa de instabilidade do movimento de balanço estaria relacionada com o momento de Munk. O momento de Munk tende a desestabilizar o veículo, colocando-o na direção perpendicular ao fluxo de água. A razão é a localização assimétrica dos pontos de estagnação (TRIANTAFYLLOU; HOVER, 2003).

Para encerrar esta seção, mostra-se o gráfico dos valores singulares na figura 5.8 do sistema em malha aberta, onde percebe-se a característica de uma função estritamente 


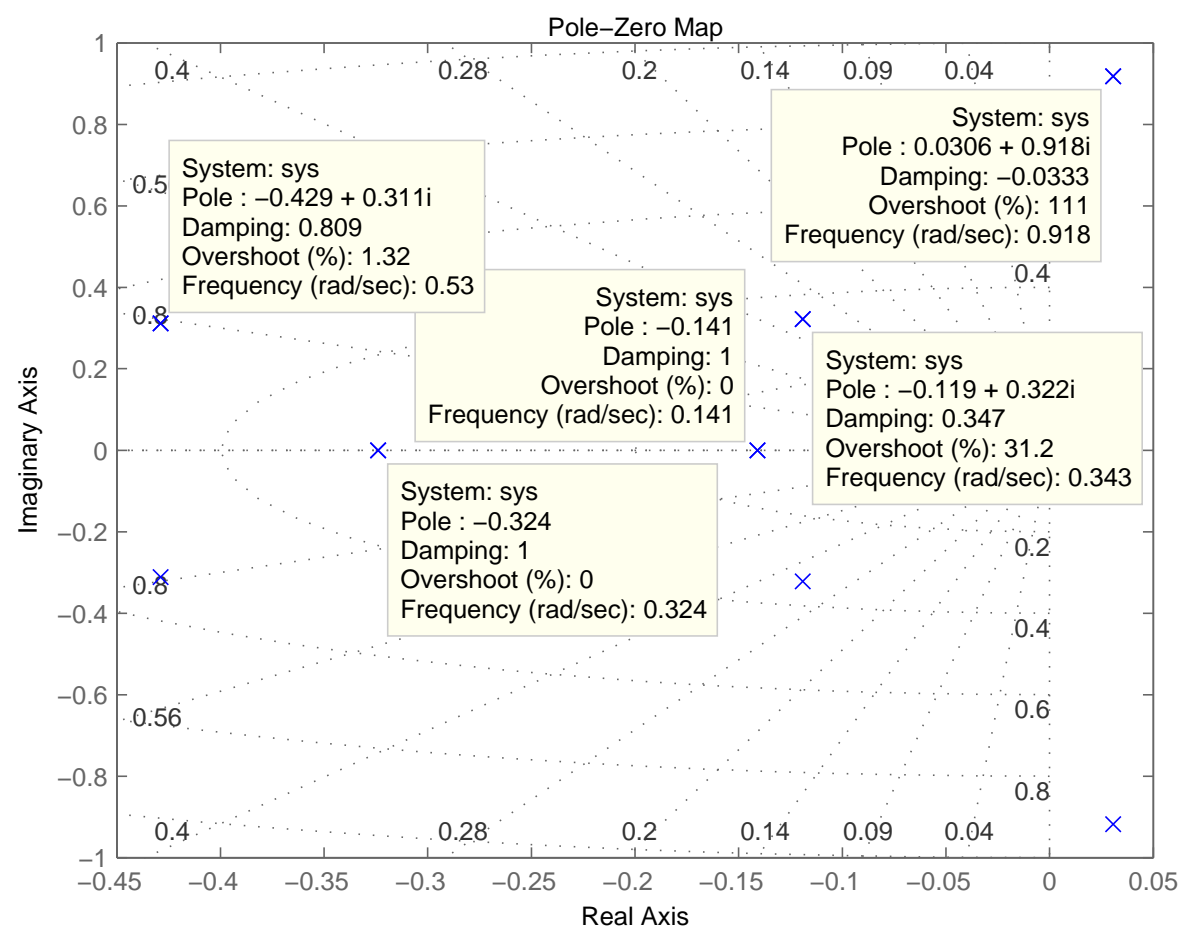

Figura 5.6: Distribuição de pólos e zeros do modelo linear do VSA.

Tabela 5.2: Autovalores e Autovetores da Planta $G(s)$

\begin{tabular}{|c|c|c|}
\hline $\begin{array}{l}\text { arfagem/caturro (oscilação) } \\
\lambda_{1,2}=-0,429 \pm 0,311 i\end{array}$ & $\begin{array}{c}\text { caturro } \\
\lambda_{3}=-0,141\end{array}$ & $\begin{array}{c}\text { balanço (oscilação) } \\
\lambda_{4,5}=0,0306 \pm 0,918 i\end{array}$ \\
\hline $\begin{array}{ll} & 0\end{array}$ & 0 & 0 \\
\hline$-2,1664 \mathrm{e}-16 \pm 4,9585 \mathrm{e}-17 \mathrm{i}$ & $-6,2755 \mathrm{e}-17$ & $-8,9708 \mathrm{e}-02 \mp 1,6625 \mathrm{e}-01 \mathrm{i}$ \\
\hline $4,2041 \mathrm{e}-01 \pm 1,0034 \mathrm{e}-01 \mathrm{i}$ & $-5,5668 \mathrm{e}-01$ & $-9,6763 \mathrm{e}-18 \mp 1,0476 \mathrm{e}-17 \mathrm{i}$ \\
\hline$-1,1654 \mathrm{e}-16 \pm 3,7910 \mathrm{e}-17 \mathrm{i}$ & $-2,0398 \mathrm{e}-17$ & $2,1952 \mathrm{e}-02 \pm 6,5807 \mathrm{e}-01 \mathrm{i}$ \\
\hline$-3,4173 \mathrm{e}-01 \pm 2,4810 \mathrm{e}-01 \mathrm{i}$ & $-1,1592 \mathrm{e}-01$ & $1,4481 \mathrm{e}-17 \mp 4,9162 \mathrm{e}-17 \mathrm{i}$ \\
\hline $4,1451 \mathrm{e}-17 \pm 3,3219 \mathrm{e}-17 \mathrm{i}$ & $6,0098 \mathrm{e}-17$ & $-2,1404 \mathrm{e}-02 \pm 1,2795 \mathrm{e}-01 \mathrm{i}$ \\
\hline 2,1997e-16 $\pm 7,1304 \mathrm{e}-17 \mathrm{i}$ & $1,4475 \mathrm{e}-16$ & 7,1691e-01 \\
\hline 7,9678e-01 & $8,2260 \mathrm{e}-01$ & $-4,2782 \mathrm{e}-17 \mp 1,4306 \mathrm{e}-17 \mathrm{i}$ \\
\hline \multicolumn{2}{|c|}{$\begin{array}{c}\text { deriva/balanço (oscilação) } \\
\lambda_{67}=-0,119 \pm 0,322 i\end{array}$} & $\begin{array}{c}\text { avanço } \\
\lambda_{8}=-0,324\end{array}$ \\
\hline \multicolumn{2}{|c|}{0} & $1,0000 \mathrm{e}+00$ \\
\hline \multicolumn{2}{|c|}{$-4,1529 \mathrm{e}-01 \pm 3,6773 \mathrm{e}-01 \mathrm{i}$} & $-3,7921 \mathrm{e}-21$ \\
\hline \multicolumn{2}{|c|}{$-9,7891 \mathrm{e}-17 \mp 4,9026 \mathrm{e}-17 \mathrm{i}$} & $-2,7193 \mathrm{e}-21$ \\
\hline \multicolumn{2}{|c|}{$-8,8926 \mathrm{e}-02 \pm 2,4010 \mathrm{e}-01 \mathrm{i}$} & $1,8945 \mathrm{e}-22$ \\
\hline \multicolumn{2}{|c|}{$3,0105 \mathrm{e}-17 \mp 7,8983 \mathrm{e}-17 \mathrm{i}$} & $1,3280 \mathrm{e}-21$ \\
\hline \multicolumn{2}{|c|}{$2,1010 \mathrm{e}-01 \pm 1,5927 \mathrm{e}-01 \mathrm{i}$} & $-5,8082 \mathrm{e}-22$ \\
\hline \multicolumn{2}{|c|}{$7,4648 \mathrm{e}-01$} & $-5,8473 \mathrm{e}-22$ \\
\hline \multicolumn{2}{|c|}{$-1,7376 \mathrm{e}-16 \pm 7,0372 \mathrm{e}-17 \mathrm{i}$} & $-4,0989 \mathrm{e}-21$ \\
\hline
\end{tabular}



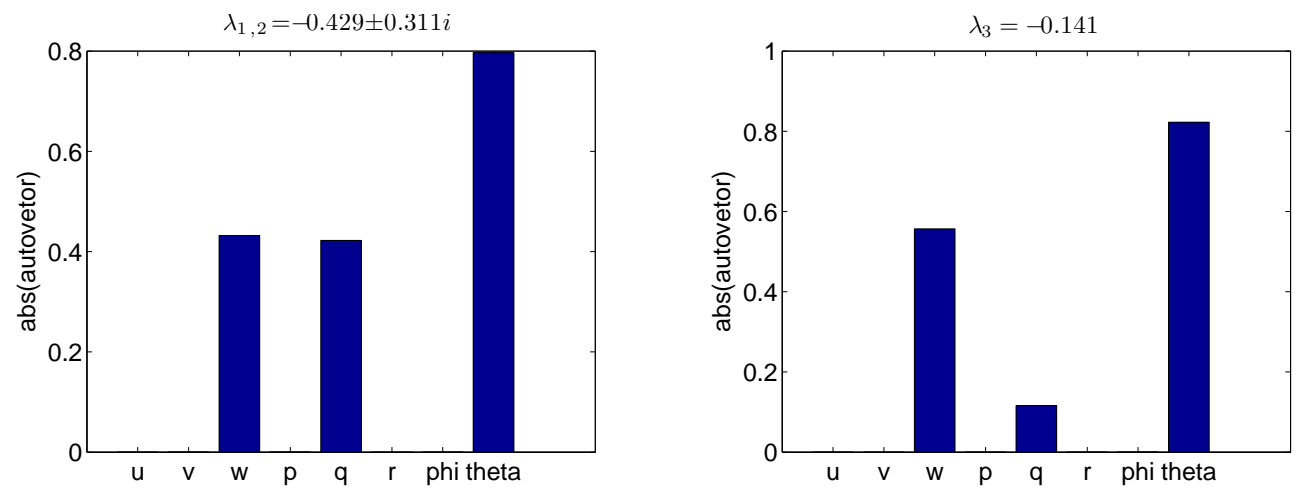

(a)

(b)
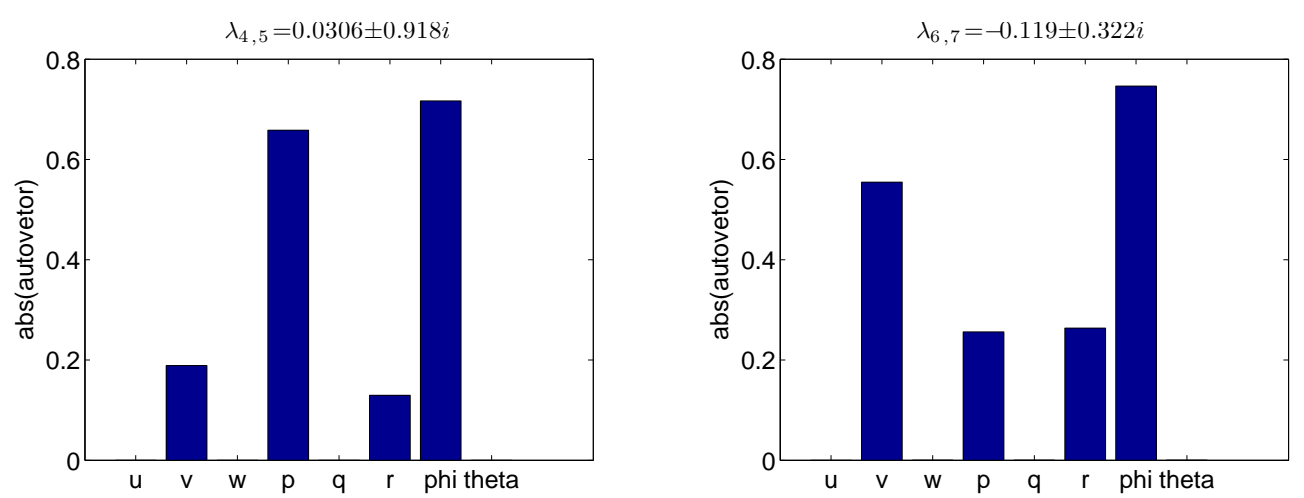

(c)

(d)

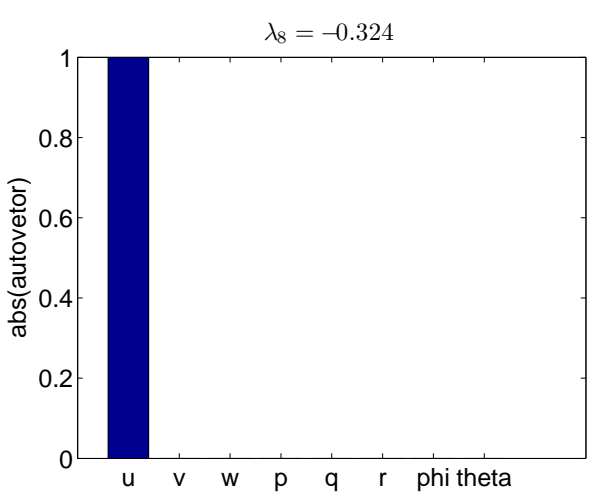

(e)

Figura 5.7: Magnitude dos autovetores associados a seus autovalores correspondentes $\lambda_{*}$ 


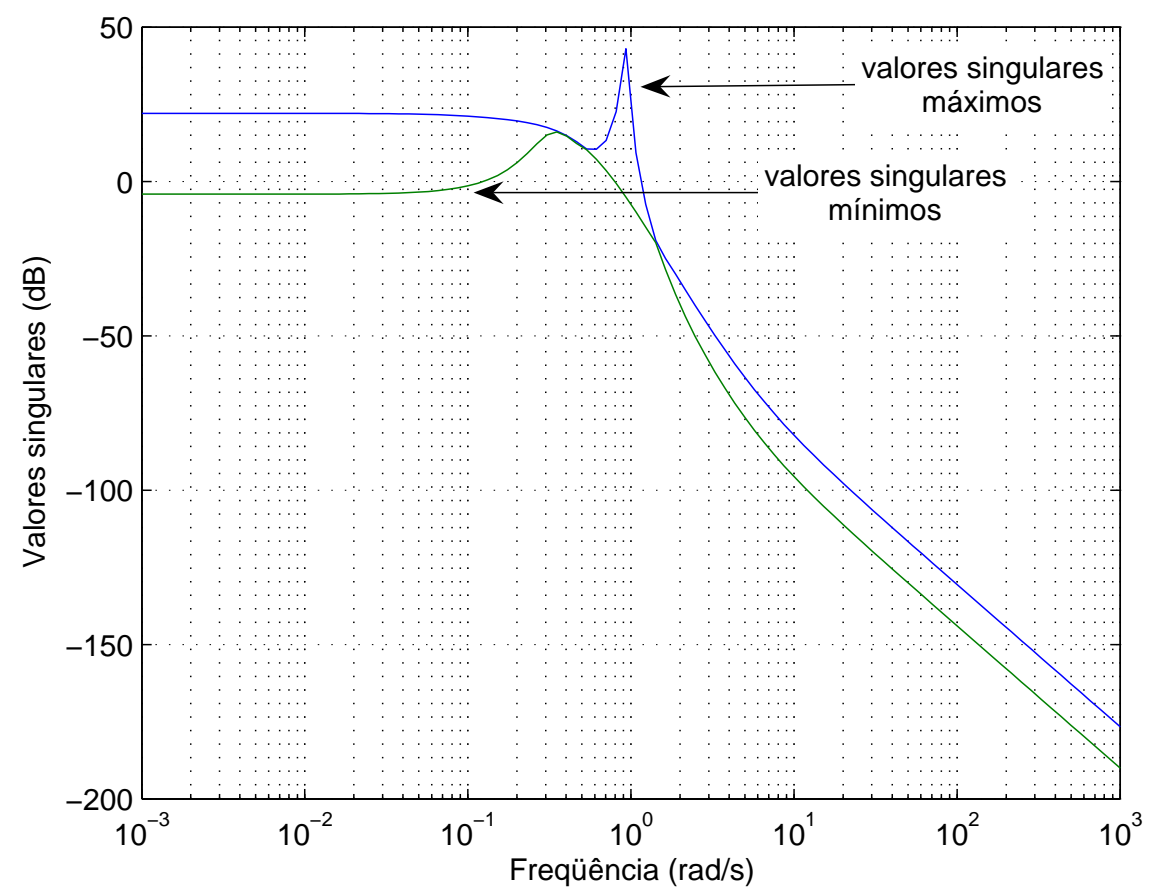

Figura 5.8: Valores Singulares de $G(s)$ em malha aberta.

própria $^{2}$, com características de um sistema bem condicionado.

\subsubsection{Normalização}

Para facilitar o projeto do controlador, as entradas do controlador são normalizadas considerando a saturação física dos atuadores: $30^{\circ}$ para o leme vertical $\left(\delta_{r}\right)$ e $25^{\circ}$ para o leme horizontal $\left(\delta_{s}\right)$. De acordo com Logan (1993), as variáveis controladas também são normalizadas considerando o erro máximo de acompanhamento do sinal de referência: $10^{\circ}$ para os ângulos de balanço $(\phi)$ e caturro $(\theta) ; 10^{\circ} / \mathrm{s}$ para a velocidade de guinada $(\dot{\psi})$; e $1 \mathrm{~m} / \mathrm{s}$ para a velocidade de $\operatorname{arfagem}(\dot{z})$. Assume-se também que o máximo valor esperado do erro para as velocidade de avanço $(u)$, deriva $(v)$ e arfagem $(w)$ é de $1 \mathrm{~m} / \mathrm{s}$; o erro máximo para as velocidades angulares de balanço $(p)$, caturro $(q)$ e guinada $(r)$ é de $10^{\circ}$ s. Portanto, as matrizes de normalização do sistema são:

$$
S_{u}=\frac{180^{\circ}}{\pi}\left[\begin{array}{cc}
1 / 30^{\circ} & 0 \\
0 & 1 / 25^{\circ}
\end{array}\right]
$$

\footnotetext{
${ }^{2}$ ver apêndice B.2
} 


$$
\begin{gathered}
S_{x}=\left[\begin{array}{cccccccc}
1 & 0 & 0 & 0 & 0 & 0 & 0 & 0 \\
0 & 1 & 0 & 0 & 0 & 0 & 0 & 0 \\
0 & 0 & 1 & 0 & 0 & 0 & 0 & 0 \\
0 & 0 & 0 & \frac{180^{\circ}}{\pi * 10} & 0 & 0 & 0 & 0 \\
0 & 0 & 0 & 0 & \frac{180^{\circ}}{\pi * 10} & 0 & 0 & 0 \\
0 & 0 & 0 & 0 & 0 & \frac{180^{\circ}}{\pi * 10} & 0 & 0 \\
0 & 0 & 0 & 0 & 0 & 0 & \frac{180^{\circ}}{\pi * 10} & 0 \\
0 & 0 & 0 & 0 & 0 & 0 & 0 & \frac{180^{\circ}}{\pi * 10}
\end{array}\right] \\
S_{y}=\frac{180^{\circ}}{\pi}\left[\begin{array}{ccccc}
\frac{1}{10} & 0 & 0 & 0 \\
0 & \frac{1}{10} & 0 & 0 \\
0 & 0 & \frac{1}{10} & 0 \\
0 & 0 & 0 & \frac{\pi}{180^{\circ} * 10}
\end{array}\right]
\end{gathered}
$$

\subsection{Síntese do Controlador $\mathcal{H}_{\infty}$}

Antes de prosseguir na síntese do controlador é indispensável que o sistema LIT atenda todas as propriedades expressas na página 33 (controlabilidade, observabilidade, estabilizabilidade e detectabilidade), além das hipóteses dadas na pagina 49. Adota-se a ilustração da figura 5.9 para a síntese do controlador $K$. Nesta, P é a Planta generalizada (veja figura 4.10) que contém também todas as funções de ponderação.

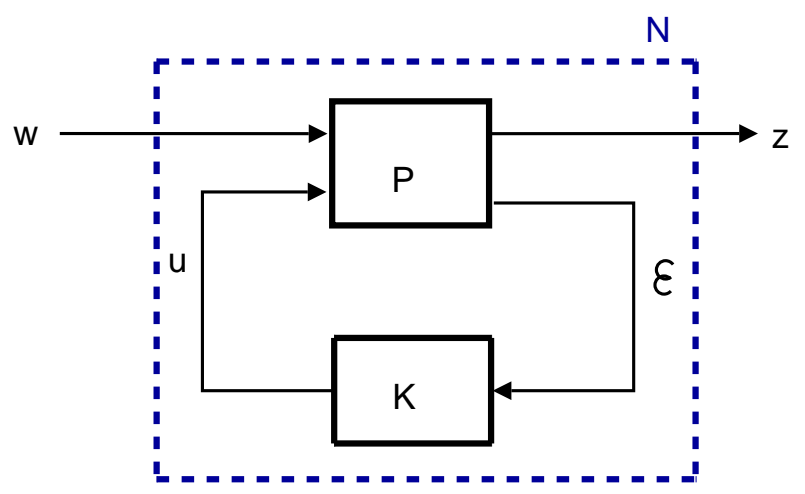

Figura 5.9: Configuração geral para síntese. 


\subsubsection{Especificações do projeto}

A seguir, mostram-se as especificações do projeto do controlador para a dinâmica do submersível.

As especificações do projeto foram sumarizadas ${ }^{3}$ nos itens seguintes:

E1 Estabilidade em malha fechada;

E2 $\bar{\sigma}(S)<1$ para $\omega<0,7 \mathrm{rad} / \mathrm{s}$;

E3 $\bar{\sigma}(T)<1$ para $\omega>2 \mathrm{rad} / \mathrm{s}$;

E4 $\bar{\sigma}(C)<1$ para $\omega>7 \mathrm{rad} / \mathrm{s} \mathrm{e}$

E5 Resposta temporal ao degrau unitário

Tabela 5.3: Resposta do sistema, assumindo um tempo de acomodação $T=4 \tau$

\begin{tabular}{rccc}
\hline $\begin{array}{r}\text { Sinal } \\
\text { controlado }\end{array}$ & $\begin{array}{c}\text { Ultrapassagem } \\
\text { percentual }\end{array}$ & $\begin{array}{c}\text { Tempo de } \\
\text { assentamento }\end{array}$ & $\mathbf{1 / \tau}$ \\
\hline \hline balanço & $20 \%$ & $20 \mathrm{~s}$ & 0,02 \\
caturro & $10 \%$ & $20 \mathrm{~s}$ & 0,02 \\
veloc. de guinada & $10 \%$ & $20 \mathrm{~s}$ & 0,02 \\
veloc. de submersão & $10 \%$ & $80 \mathrm{~s}$ & 0,005 \\
\hline
\end{tabular}

Como o caso é multivariável, as especificações de E1 a E4 foram iguais para todos os sinais a serem controlados, isto diminui o esforço na busca de parâmetros para as matrizes de ponderação. Após determinar um valor $\gamma$ sub-ótimo na síntese do controlador, e verificase a melhor formatação para $S, T$ e $C$; E5 é necessária para testar as especificações de desempenho do sistema no domínio do tempo.

\subsubsection{Funções de ponderação}

As funções que ponderarão os sinais exógenos $W_{i}, W_{d}, W_{n}$ são inicialmente definidas como matrizes identidade $I$. Porém, $R$ pode ser formatada como um filtro passa-baixa no final do processo, para reduzir o sobresinal na resposta do sistema controlado.

A escolha adequada de $W_{S}, W_{C}$ e $W_{T}$ permitirá alcançar os requisitos de Robustez para

\footnotetext{
${ }^{3} \mathrm{Na}$ seção 4.7 encontram-se bem detalhadas as especificações do projeto do controlador, que vão permitir formatar $S, T$ e $C$ para conseguir ER e DR
} 
o sistema (ER e DR). A busca de parâmetros envolvidos é complexa é fatigante devido ao grande número de parâmetros envolvidos. A recomendação é utilizar um procedimento de otimização, através de uma busca pelo método gradiente, algoritmos genéticos, ou um outro procedimento.

Neste trabalho, $W_{S}$ e $W_{T}$ foram definidas pelo método descrito em (DONHA; KATEBI, 2004) que utiliza algoritmos genéticos. $W_{C}$ foi eleita de acordo com a equação (4.41), modificada para o caso multivariável, ver figuras 5.10, 5.12 e 5.11.

As funções de ponderação usadas foram:

$$
\begin{aligned}
& W_{S_{\phi}}=\frac{s^{3}+6.91 s^{2}+2.76 s+8.3}{s^{3}+14.04 s^{2}+19.95 s+19.43} \\
& W_{S_{\theta}}=\frac{s^{3}+4.57 s^{2}+3.72 s+3.61}{s^{3}+8.38 s^{2}+10.63 s+4.81} \\
& W_{S_{\psi}}=\frac{s^{3}+1.32 s^{2}+2.37 s+4.12}{s^{3}+19.98 s^{2}+11.75 s+12.91} \\
& W_{S_{\dot{z}}}=\frac{s^{3}+11.2 s^{2}+14.46 s+7.51}{s^{3}+12.9 s^{2}+18.81 s+11.93} \\
& W_{C_{\delta_{r}}}=\frac{s^{2}+3.44 s+2.96}{1 \mathrm{e}-8 s^{2}+3.20 \mathrm{e}-3 s+2.562} \\
& W_{C_{\delta_{s}}}=\frac{s^{2}+3.44 s+2.96}{1 \mathrm{e}-8 s^{2}+3.20 \mathrm{e}-3 s+2.562} \\
& W_{T_{\phi}}=\frac{0.29}{7.12 s+1} \\
& W_{T_{\theta}}=\frac{0.74}{10.14 s+1} \\
& W_{T_{\dot{\psi}}}=\frac{0.35}{8.83 s+1} \\
& W_{T_{\dot{z}}}=\frac{0.67}{13.88 s+1} \\
& W_{S}=\left[\begin{array}{cccc}
W_{S_{\phi}} & 0 & 0 & 0 \\
0 & W_{S_{\theta}} & 0 & 0 \\
0 & 0 & W_{S_{\dot{\psi}}} & 0 \\
0 & 0 & 0 & W_{S_{\dot{z}}}
\end{array}\right] \quad W_{T}=\left[\begin{array}{cccc}
W_{T_{\phi}} & 0 & 0 & 0 \\
0 & W_{T_{\theta}} & 0 & 0 \\
0 & 0 & W_{T_{\dot{\psi}}} & 0 \\
0 & 0 & 0 & W_{T_{\dot{z}}}
\end{array}\right] \\
& W_{C}=\left[\begin{array}{cc}
W_{C_{\delta_{r}}} & 0 \\
0 & W_{C_{\delta_{s}}}
\end{array}\right]
\end{aligned}
$$

As figuras 5.10-5.11 mostram as funções de ponderação utilizadas na síntese do controlador.

Colocando-se o sistema na configuração de dois portos e usando as ponderações acima, o controlador $K$ pode ser encontrado através de softwares de prateleira como o Matlab ou 


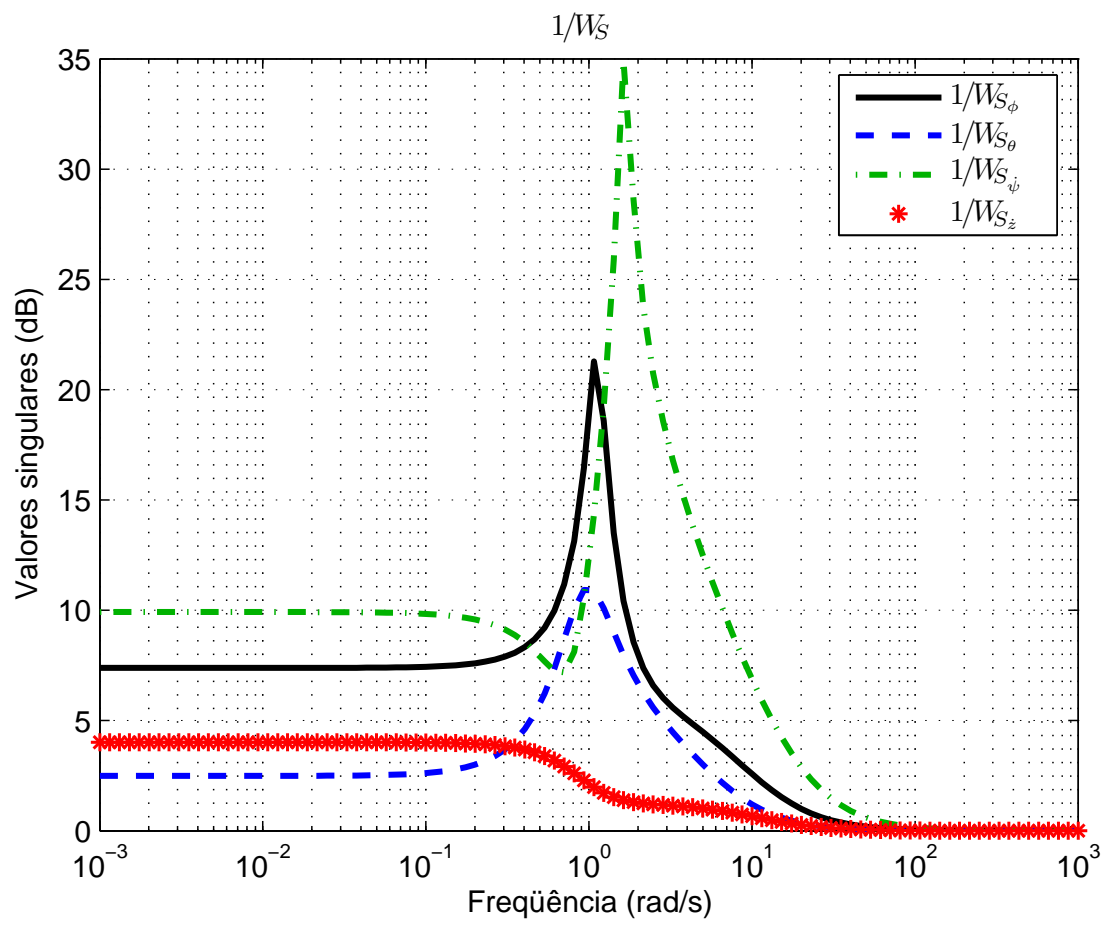

Figura 5.10: Matriz de transferência da função de ponderação $\left(1 / W_{S}\right)$. o Scilab. 


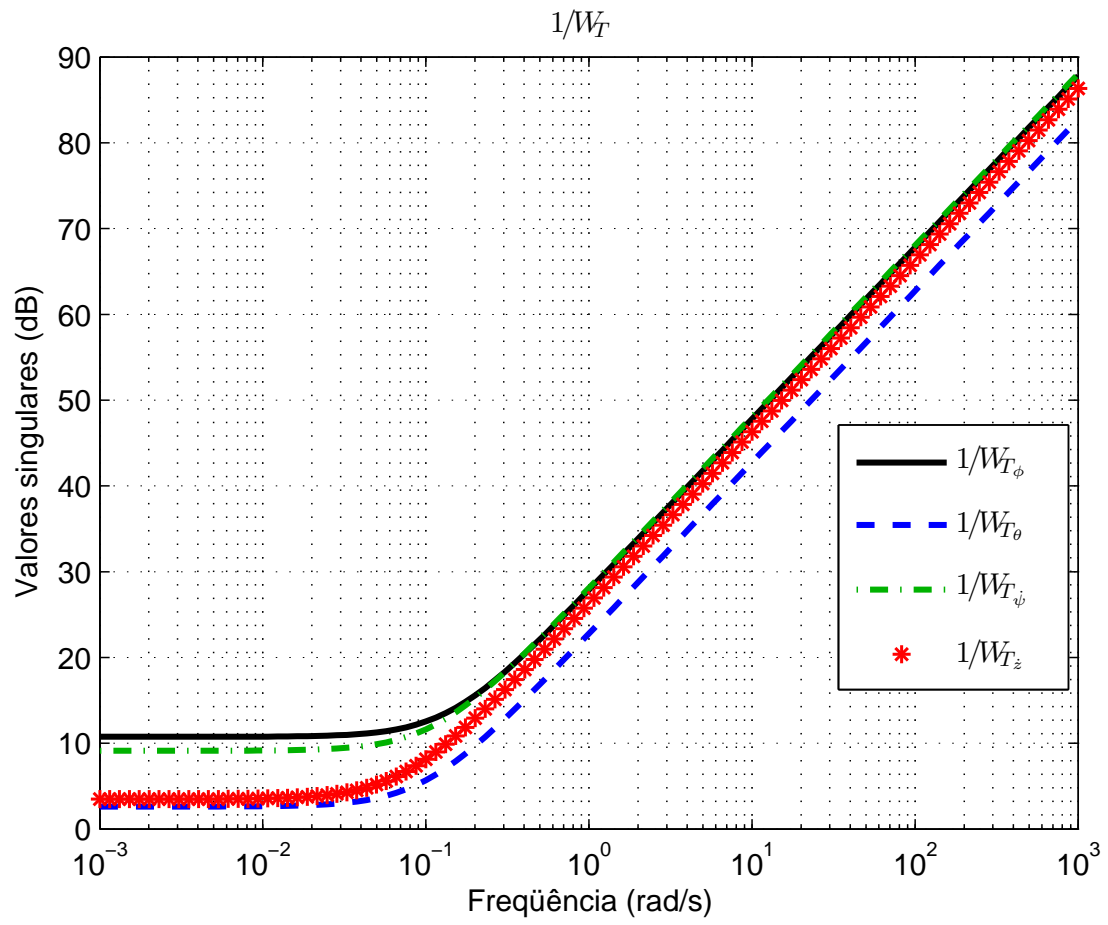

Figura 5.11: Matriz de transferência da função de ponderação $\left(1 / W_{T}\right)$.

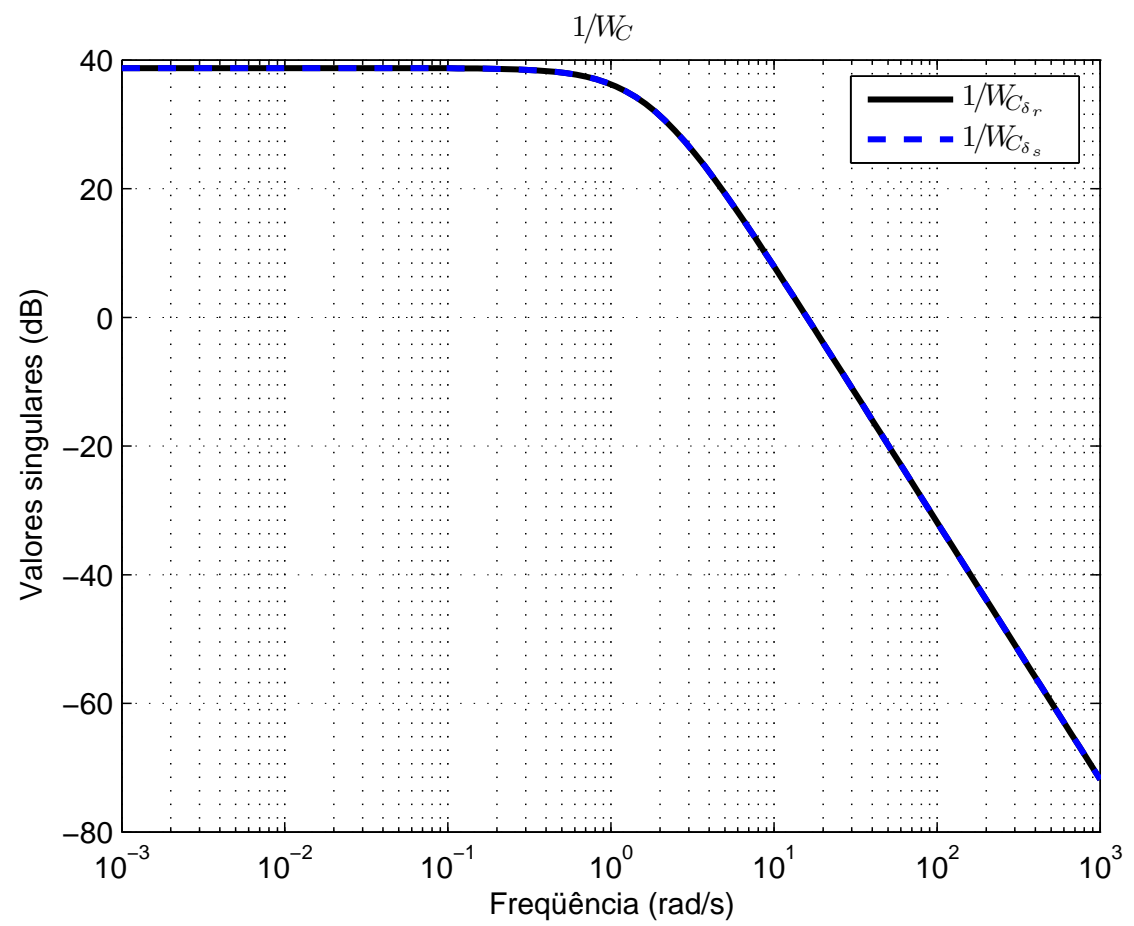

Figura 5.12: Matriz de transferência da função de ponderação $\left(1 / W_{C}\right)$. 


\subsubsection{Funções de Sensibilidade}

A figura 5.13 ilustra a função de sensibilidade $(S)$ para o sistema controlado, e $W_{S}$ a respectiva função de ponderação.

A figura 5.14 ilustra a função de sensibilidade complementar $(T)$ para o sistema controlado, e $W_{T}$ a respectiva função de ponderação.

A figura 5.15 ilustra a função de sensibilidade do controlador $(C)$ para o sistema controlado, e $W_{C}$ a respectiva função de ponderação.

As figuras 5.16, 5.17 e 5.18 mostram a resposta do sistema após de ser aplicada a ponderação, e permitem avaliar a Robustez do sistema controlado.

Na tabela 5.4 mostram-se alguns parâmetros importantes que auxiliam o entendimento das respostas do sistema controlado (figuras 5.13, 5.14 e 5.15). Na tabela, $f_{r}$ é a freqüência de cruzamento que corresponde ao ganho de $0 \mathrm{~dB}$ da resposta, e $f_{c}$ é a freqüência de corte que corresponde ao ganho da resposta diminuído de $3 \mathrm{~dB}$.

Tabela 5.4: Funções de Sensibilidade do sistema controlado

\begin{tabular}{cllll}
\hline Sensibilidade & $\begin{array}{l}\text { Freqüência de } \\
\text { cruzamento }\left(f_{r}\right)\end{array}$ & $\begin{array}{l}\text { Freqüência de } \\
\text { corte }\left(f_{c}\right)\end{array}$ & Sobresinal & Decaimento \\
\hline \hline$S_{\phi}$ & - & - & $15,66 \mathrm{~dB}$ & - \\
$S_{\theta}$ & - & - & $4,17 \mathrm{~dB}$ & - \\
$S_{\dot{\psi}}$ & $0,7 \mathrm{rad} / \mathrm{s}$ & $0,6 \mathrm{rad} / \mathrm{s}$ & - & - \\
$S_{\dot{z}}$ & - & $3 \mathrm{rad} / \mathrm{s}$ & - & - \\
\hline$T_{\phi}$ & $2,5 \mathrm{rad} / \mathrm{s}$ & $2,88 \mathrm{rad} / \mathrm{s}$ & $16 \mathrm{~dB}$ & $-110 \mathrm{~dB} /$ década \\
$T_{\theta}$ & $1 \mathrm{rad} / \mathrm{s}$ & $1,26 \mathrm{rad} / \mathrm{s}$ & $0,5 \mathrm{~dB}$ & $-110 \mathrm{~dB} /$ década \\
$T_{\dot{\psi}}$ & - & $1,58 \mathrm{rad} / \mathrm{s}$ & $-162,5 \mathrm{~dB}$ & $-60 \mathrm{~dB} /$ década \\
$T_{\dot{z}}$ & - & $0,06 \mathrm{rad} / \mathrm{s}$ & - & $-30 \mathrm{~dB} /$ década \\
\hline$C_{\delta_{r}}$ & $6,5 \mathrm{rad} / \mathrm{s}$ & $10 \mathrm{rad} / \mathrm{s}$ & $12,5 \mathrm{~dB}$ & $-84 \mathrm{~dB} /$ década \\
$C_{\delta_{s}}$ & $3,98 \mathrm{rad} / \mathrm{s}$ & $8,32 \mathrm{rad} / \mathrm{s}$ & $1,6 \mathrm{~dB}$ & $-75 \mathrm{~dB} /$ década \\
\hline
\end{tabular}

Da sensibilidade (S) verifica-se que E2 não foi garantida para o balanço e o caturro (figuras 5.13(a) e 5.13(b)); ao contrário, E2 foi garantida para as velocidades de guinada e submersão (figuras 5.13(c) e 5.13(d)). Da sensibilidade complementar (T) observa-se que E3 foi garantida para todos os sinais controlados (figura 5.14). É também importante observar que os sinais relativos às velocidades de guinada e submersão (figuras 5.13(c) e $5.14(\mathrm{~d}))$ estão abaixo de $-150 \mathrm{~dB}, \forall \omega$, o que provavelmente vai degradar o tracking. Da sensibilidade do controlador $(C)$ observa-se que as freqüências de cruzamento (roll off) são de $6,5 \mathrm{rad} / \mathrm{s}$ e $3,98 \mathrm{rad} / \mathrm{s}$; isto garante $\mathbf{E 4}$.

Note-se que $S$ demostra pouca ação de rejeição de distúrbios nos sinais $\phi$ e $\theta$, porém, $S$ é 
pequena em baixas freqüências para os sinais de velocidade $\dot{\psi}$ e $\dot{z}$, quer dizer, a rejeição a distúrbios é maior nesta banda. Naturalmente, pode-se dedicar um maior esforço na busca de parâmetros da função de ponderação $W_{s}$ e provavelmente aí reside a maior dificuldade a ser enfrentada por projetistas que utilizam esta técnica.

A sensibilidade complementar $T$ apresenta um decaimento característico em alta freqüência. Isto é mais notório nas variáveis controladas $\phi$ e $\theta$, ilustradas nas figuras 5.14(a) e 5.14(b), respectivamente. Este bom comportamento está associado à estabilidade robusta, e à rejeição de ruídos do sensor. Porém, observa-se um sobresinal na resposta de balanço próximo a 1rad/s com uma amplitude de 16dB. Este fato deve-se a que o veículo é sub-atuado, e o efeito na resposta temporal é o sobresinal grande.

A forma de $C=K S$ tem um comportamento característico, já que limita a ação de controle em altas freqüências, reduzindo seu valor progressivamente a partir da freqüência de cruzamento.

A análise das figuras 5.16, 5.17 e 5.18, mostra a boa ponderação realizada no sistema, já que as amplitudes são menores do que 1 na norma $\mathcal{H}_{\infty}$ e validam a Robustez do sistema controlado. As amplitudes dos sinais na figura 5.16, relativas ao desempenho robusto $(\mathrm{DR})^{4}$, são próximas de 1 para $\omega$ em alta freqüência; isto pode ocasionar a degradação do desempenho e será avaliado na resposta temporal do sistema. As amplitudes na figura 5.17 são menores de 1 em toda a faixa de freqüências, isto garante a estabilidade robusta do sistema (ER).

${ }^{4}$ Para DR, veja equação 4.38 


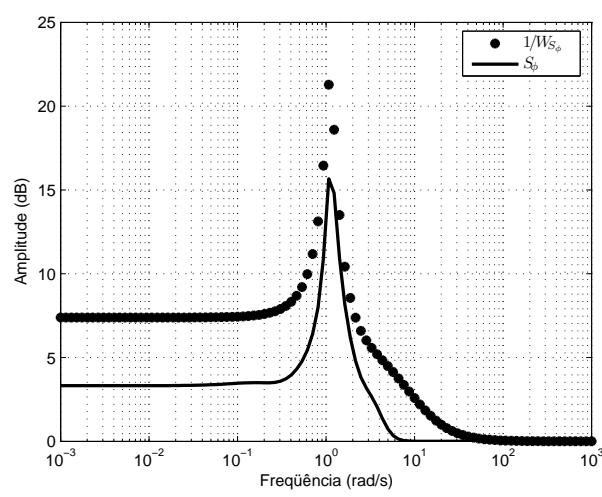

(a) balanço

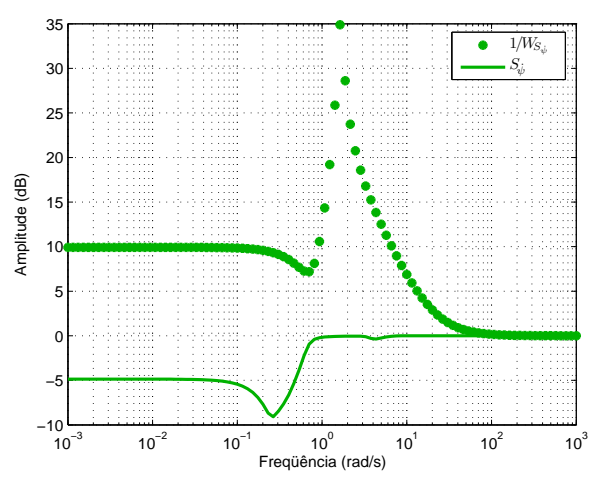

(c) velocidade de guinada

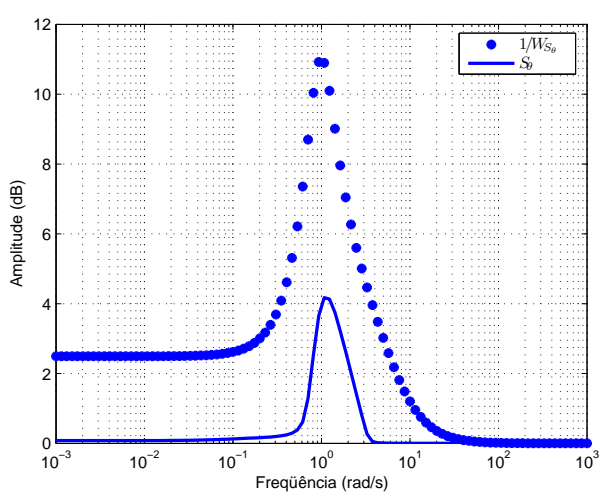

(b) caturro

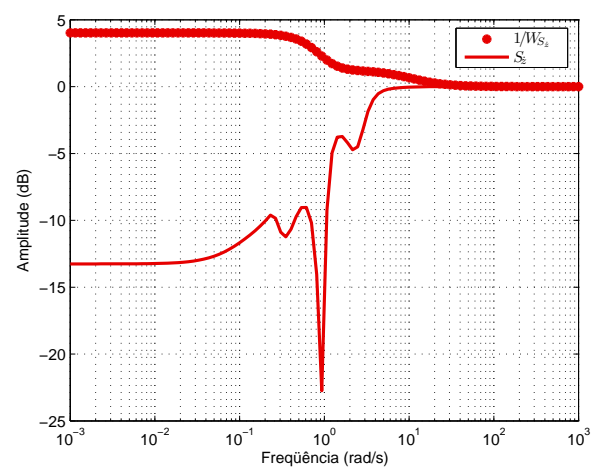

(d) velocidade de submersão

Figura 5.13: Função de sensibilidade $(S)$ e inversa da função de ponderação $\left(1 / W_{S}\right)$ 


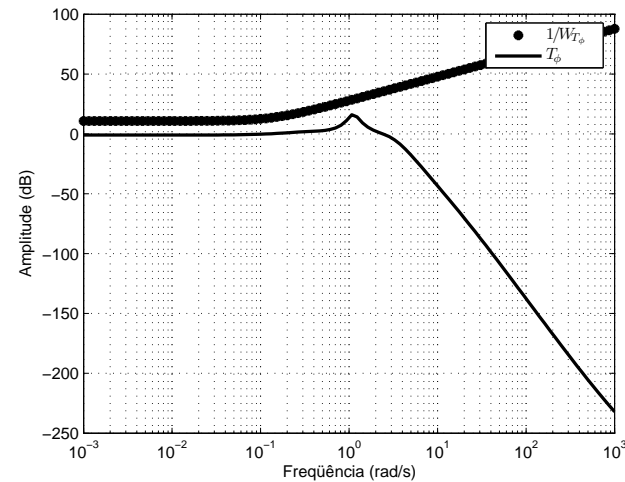

(a) balanço

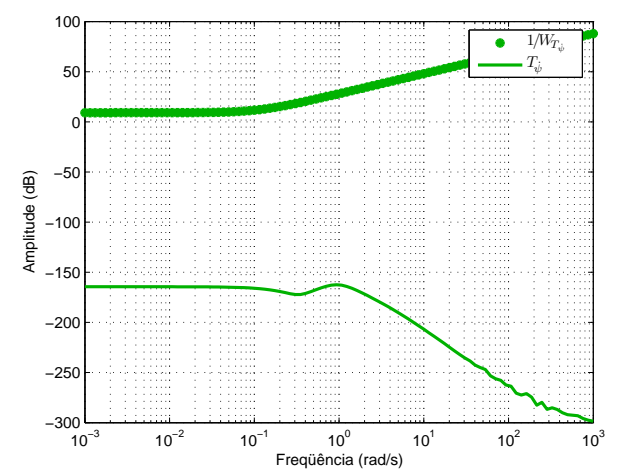

(c) velocidade de guinada

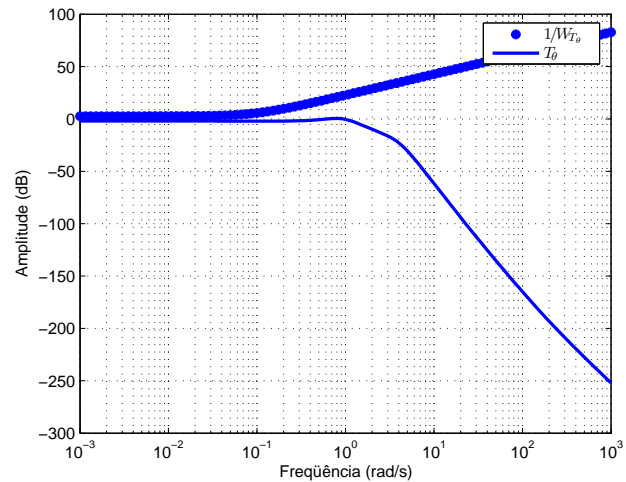

(b) caturro

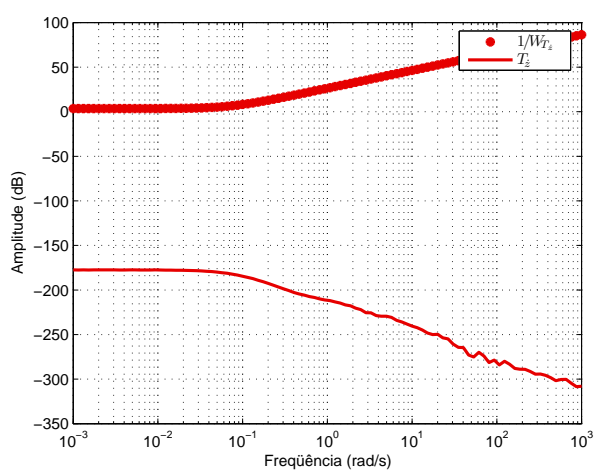

(d) velocidade de submersão

Figura 5.14: Função de sensibilidade complementar $(T)$ e inversa da função de ponderação $\left(1 / W_{T}\right)$

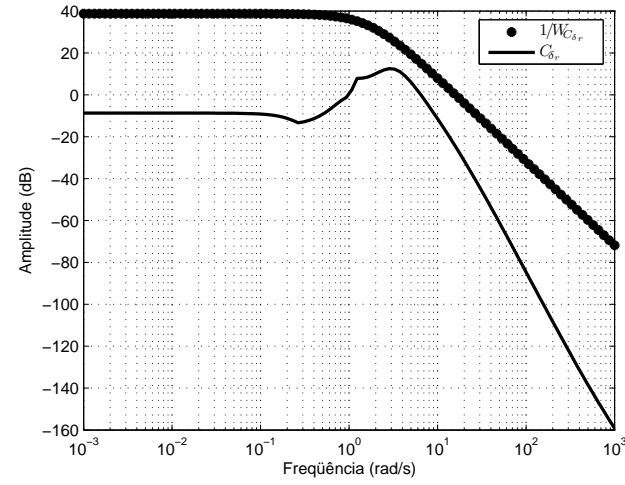

(a) leme vertical

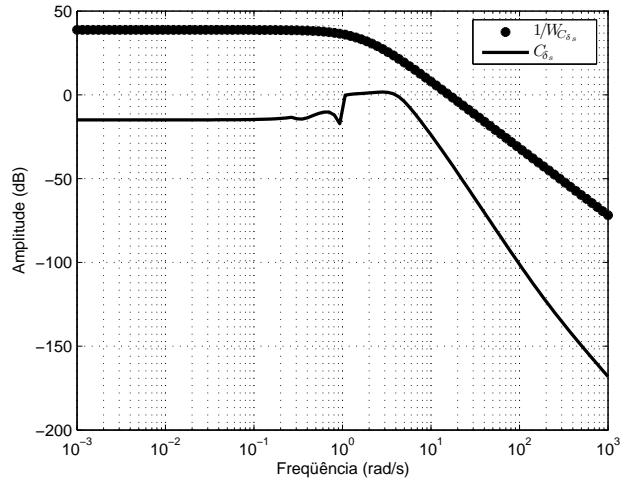

(b) leme horizontal

Figura 5.15: Função de sensibilidade do controlador $(C)$ e inversa da função de ponderação $\left(1 / W_{C}\right)$ 


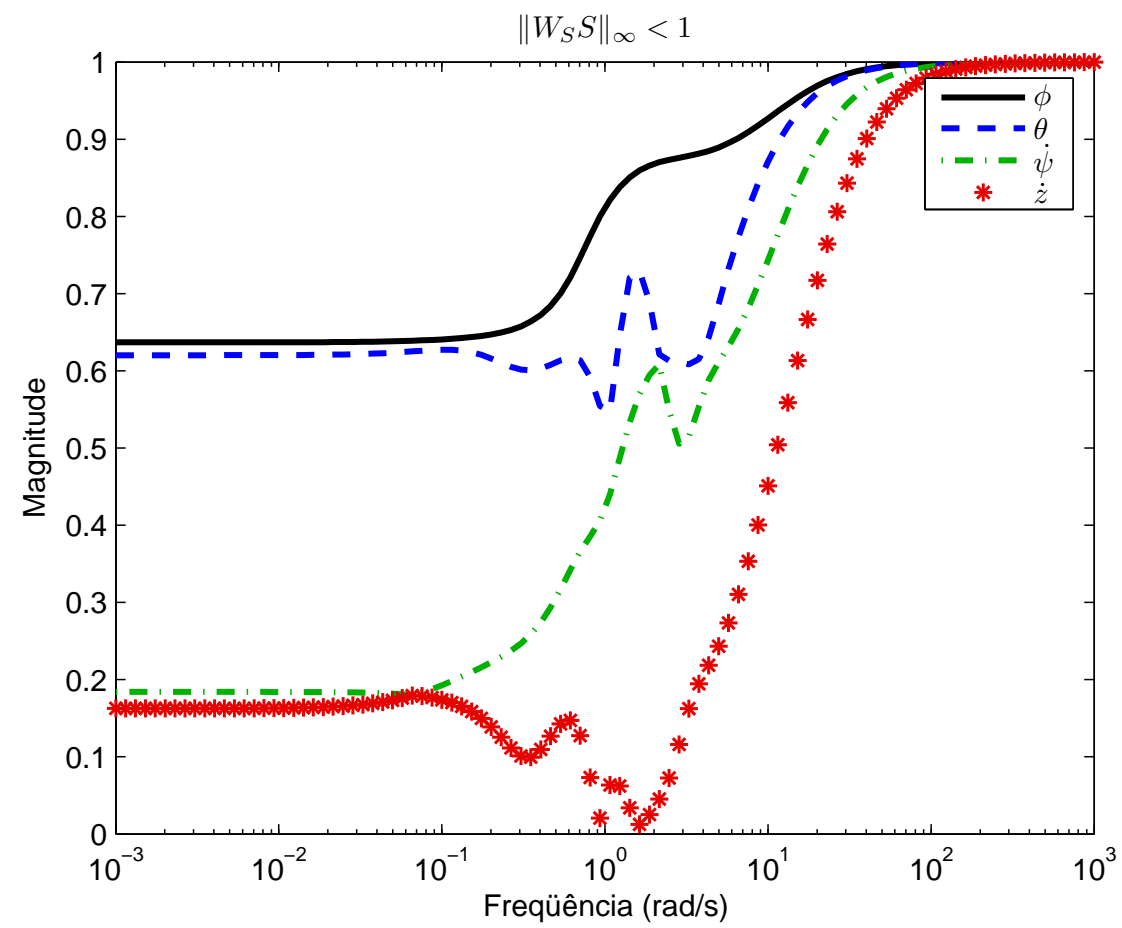

Figura 5.16: Matriz de Transferência $W_{S} S$.

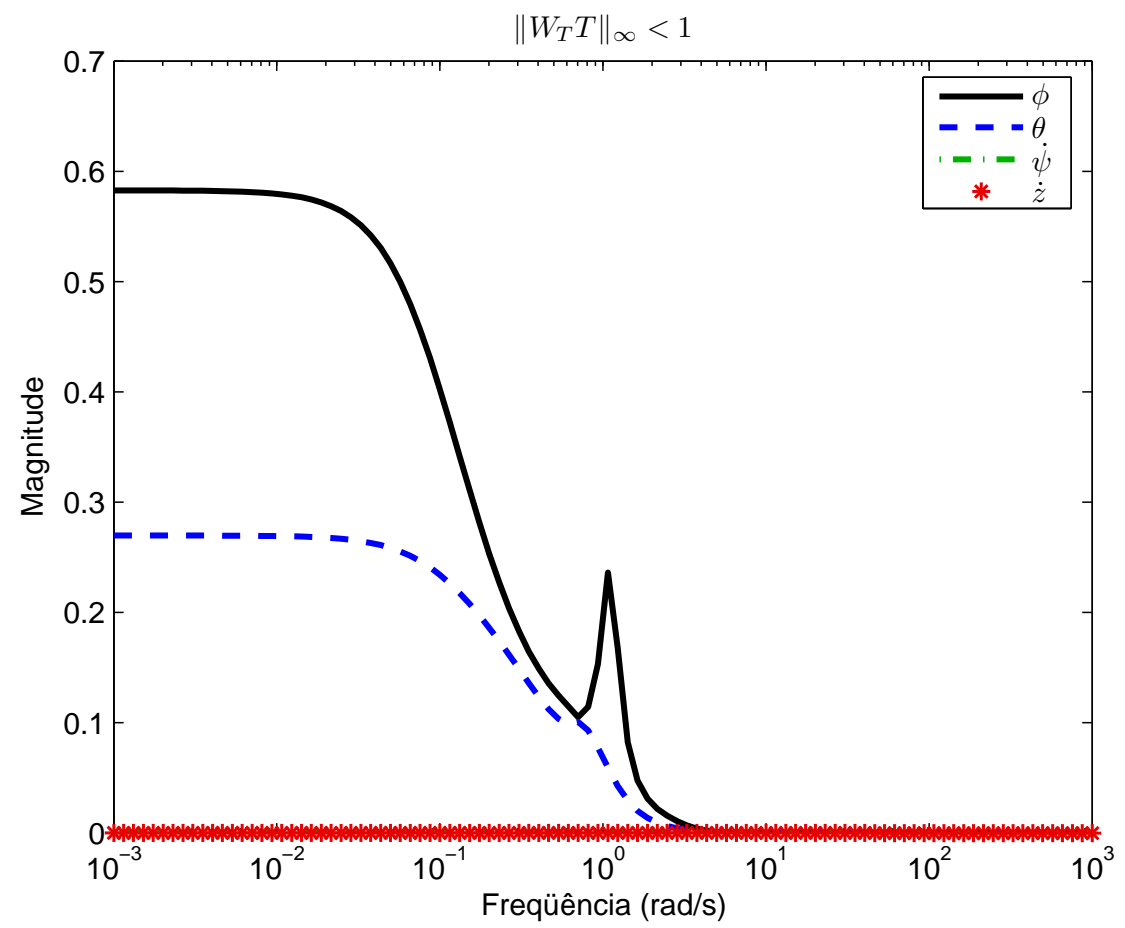

Figura 5.17: Matriz de Transferência $W_{T} T$. 


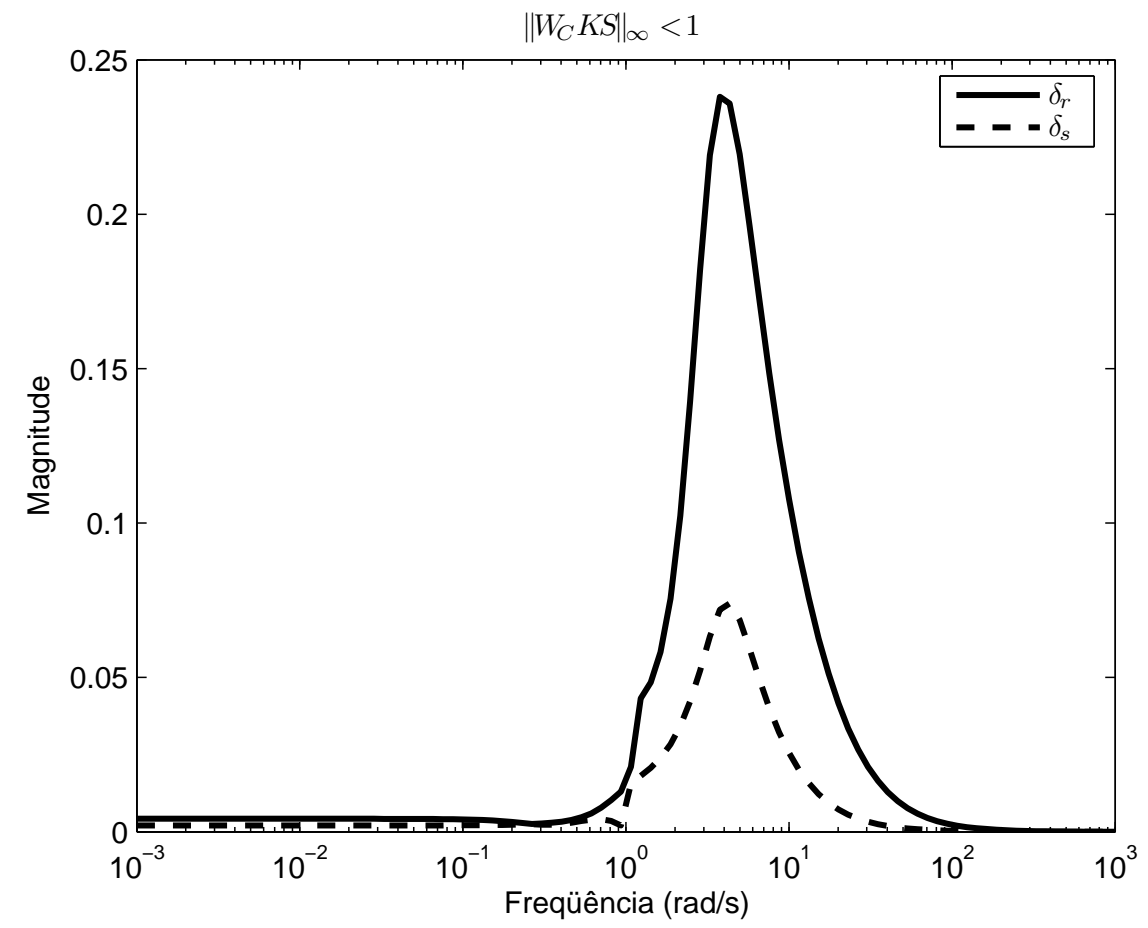

Figura 5.18: Matriz de Transferência $W_{C} C$.

\subsubsection{Resposta Temporal}

A resposta temporal do sistema é analisada tanto para o modelo linear quanto para o modelo não linear, com especial ênfase no último caso.

Pode-se obter grande quantidade de resultados, já que foi desenvolvido o modelo não linear completo de seis graus de liberdade para o veículo submersível autônomo. A seguir, apresentam-se os gráficos que se consideram mais relevantes na análise do sistema controlado.

\subsubsection{Resposta Linear}

Após a síntese do controlador $K$, o sistema controlado é testado com sinais de entrada para cada canal $(\phi, \theta, \dot{\psi}$ ou $\dot{z})$. Assim, na resposta do sistema pode-se observar características como: estabilidade, acompanhamento do sinal de entrada (referência), sobresinal, tempo de resposta e os acoplamentos que existem entre as variáveis controladas.

Na figura 5.19 mostram-se quatro respostas para o sistema linear controlado. Na resposta para o balanço (figura 5.19(a)), um sinal degrau foi injetado na entrada balanço e foram obtidas quatro respostas, uma relativa ao mesmo balanço e as outras três (caturro, velocidade de guinada e velocidade de submersão) relativas ao acoplamento existente no 
sistema. Na resposta para o caturro (figura 5.19(b)), um sinal degrau foi injetado no caturro e foram também obtidas quatro respostas, uma relativa ao mesmo caturro e as outras três (balanço, velocidade de guinada e velocidade de submersão) relativas ao acoplamento existente no sistema. Na resposta para a velocidade de guinada (figura 5.19(c)), um sinal degrau foi injetado na velocidade de guinada e foram também obtidas quatro respostas, uma relativa à mesma velocidade de guinada e as outras três (balanço, caturro e a velocidade de submersão) relativas ao acoplamento existente no sistema. Na resposta para a velocidade de submersão (figura 5.19(d)), um sinal degrau foi injetado na velocidade de submersão e foram também obtidas quatro respostas, uma relativa à mesma velocidade de submersão e as outras três (balanço, caturro e a velocidade de guinada) relativas ao acoplamento existente no sistema.

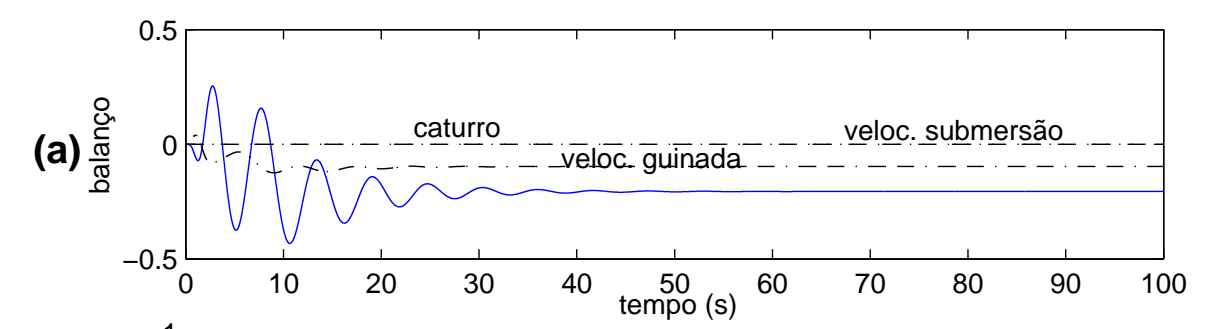

(b) 产

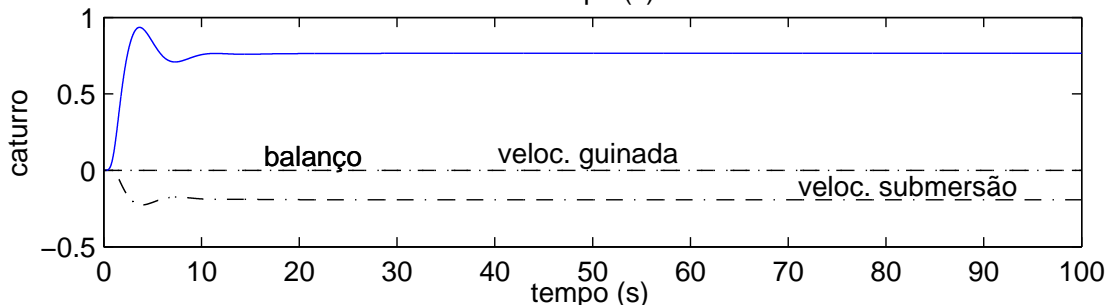

(c)

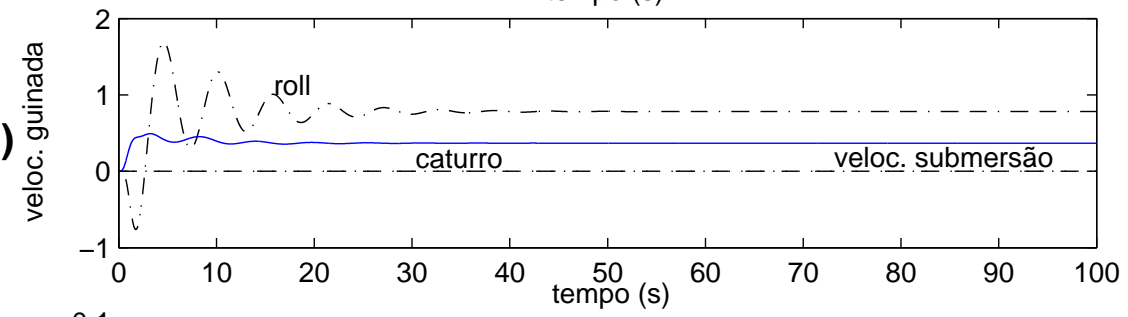

(d)

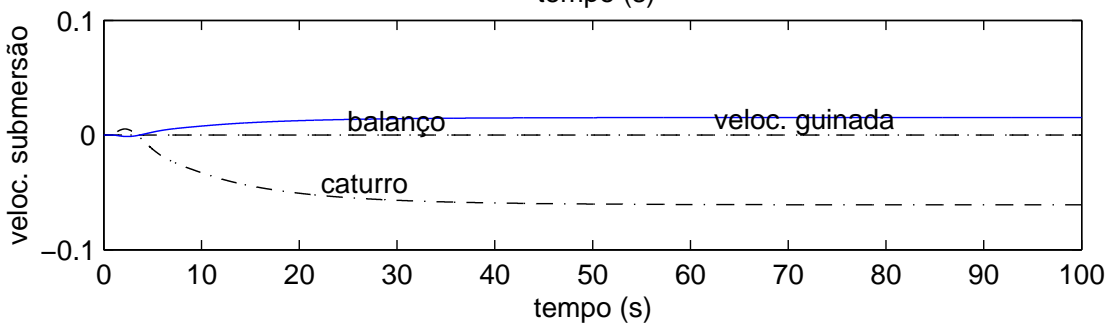

Figura 5.19: Resposta ao degrau unitário do modelo linear controlado: balanço, caturro, velocidade de guinada e velocidade de submersão. 
Dos resultados antes descritos, a resposta para o balanço $(\phi)$ tem comportamento oscilatório, isto reflete o pico que existe no gráfico de sensibilidade complementar $(T)$

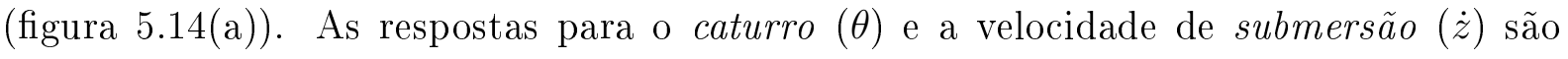
acopladas devido à configuração do veículo e a disposição de atuadores. A resposta para a velocidade de guinada $(\dot{\psi})$ também é fortemente acoplada com o balanço do veículo $(\phi)$. O sistema controlado reflete boa estabilidade e bom tracking, embora o erro no regime permanente seja grande. Esse erro é de 0,25 para a resposta de caturro, e é relativamente menor comparado com as outras respostas. A figura 5.17 mostra também a característica de bom tracking no sistema, com picos ligeiros para o balanço.

\subsubsection{Resposta Modelo Não Linear}

A resposta do modelo não linear controlado foi obtida através de uma malha fechada entre o controlador $K$ e o modelo não linear completo (seis graus de liberdade).

Foram obtidas quatro respostas (balanço, caturro, velocidade de guinada e velocidade de submersão). Estas respostas foram obtidas de modo similar ao caso linear, excitando as entradas do sistema com um sinal degrau em cada canal e observando as quatro saídas do sistema.

Na resposta para o balanço, a entrada do sistema não linear controlado é $\left[\begin{array}{llll}\phi & \theta & \dot{\psi} & \dot{z}\end{array}\right]^{T}$ $=\left[\begin{array}{llll}1 & 0 & 0 & 0\end{array}\right]^{T}$, onde 1 e 0 representam o sinal degrau unitário e o sinal zero, respectivamente. A figura 5.20(a) mostra a resposta não linear do sistema controlado.

Na resposta para o caturro, a entrada do sistema não linear controlado é $\left[\begin{array}{llll}\phi & \theta & \dot{\psi} & \dot{z}\end{array}\right]^{T}$ $=\left[\begin{array}{llll}0 & 1 & 0 & 0\end{array}\right]^{T}$, e a figura 5.20(b) mostra a saída do sistema não linear controlado.

Na resposta para a velocidade de guinada, a entrada do sistema controlado é $\left[\begin{array}{llll}\phi & \theta & \dot{\psi} & \dot{z}\end{array}\right]^{T}$ $=\left[\begin{array}{llll}0 & 0 & 1 & 0\end{array}\right]^{T}$, e a figura 5.20(c) mostra a saída do sistema não linear controlado.

Na resposta para a velocidade de submersão, a entrada do sistema não linear controlado é $\left[\begin{array}{llll}\phi & \theta & \dot{\psi} & \dot{z}\end{array}\right]^{T}=\left[\begin{array}{llll}0 & 0 & 0 & 1\end{array}\right]^{T}$, e a figura 5.20(d) mostra a saída do sistema não linear controlado.

O comportamento do modelo não linear controlado é ilustrado na figura 5.20. Estes resultados são similares aos resultados do modelo linear (figura 5.19). Cabe a mesma interpretação e certifica-se a aproximação bem feita do modelo não linear pelo modelo linear e, principalmente, valida-se o controlador $K$ para o modelo não linear.

A resposta para a velocidade de guinada (modelo não linear) apresentou mais dois acoplamentos ligeiros com o movimento de caturro e com a velocidade de submersão, veja figuras 5.19(c) e 5.20(c) para comparação. 

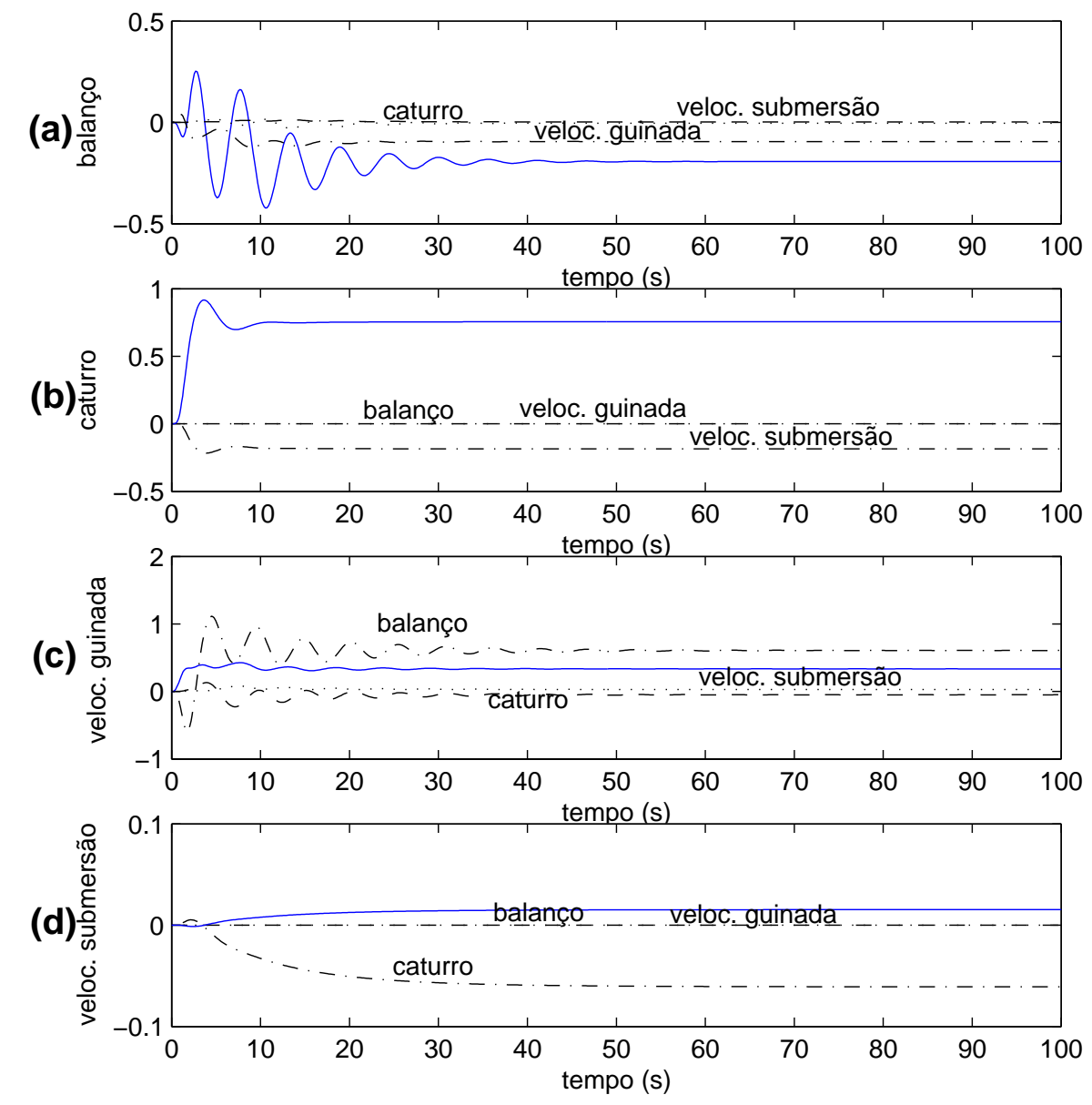

Figura 5.20: Resposta ao degrau unitário do modelo não linear controlado: balanço, caturro, velocidade de guinada e velocidade de submersão. 
Para avaliar a Robustez do Sistema Controlado, foram feitas simulações com pontos distintos de operação, usando-se o mesmo controlador projetado. A seguir, apresentam-se duas situações para as mesmas condições iniciais, porém, com velocidades diferentes do ponto de operação. A resposta para o caturro foi avaliada nos três casos.

\section{Caso 1}

Ponto de operação $x=[2,7 \mathrm{~m} / \mathrm{s}, 0,0,0,0,0,0,0]^{T}$.

Condições iniciais do sistema $x_{0}=[2 \mathrm{~m} / \mathrm{s}, 0,0,0,0,0,0,0]^{T}$

Sinal de referência, degrau unitário para o caturro $\left(\left[\begin{array}{llll}\phi & \theta & \dot{\psi} & \dot{z}\end{array}\right]^{T}=\left[\begin{array}{llll}0 & 1 & 0 & 0\end{array}\right]^{T}\right)$.

A figura 5.21(a) mostra a resposta para o caturro, e a figura 5.22 mostra as velocidades e posições do veículo controlado.

\section{Caso 2}

Ponto de operação $x=[1,1 \mathrm{~m} / \mathrm{s}, 0,0,0,0,0,0,0]^{T}$.

Condições iniciais do sistema $x_{0}=[2 \mathrm{~m} / \mathrm{s}, 0,0,0,0,0,0,0]^{T}$

Sinal de referência, degrau unitário para o caturro $\left(\left[\begin{array}{llll}\phi & \theta & \dot{\psi} & \dot{z}\end{array}\right]^{T}=\left[\begin{array}{llll}0 & 1 & 0 & 0\end{array}\right]^{T}\right)$.

A figura 5.21(b) mostra a resposta para o caturro, e a figura 5.23 mostra as velocidades e posições do veículo controlado.

\section{Caso 3}

Ponto de operação $x=[2,7 \mathrm{~m} / \mathrm{s}, 0,0,0,0,0,0,0]^{T}$.

Condições iniciais do sistema $x_{0}=[2 \mathrm{~m} / \mathrm{s}, 0,0,0,0,0,0,0]^{T}$

Sinal de referência, degrau para o caturro $^{5}$.

No modelo não linear modificaram-se propositalmente três coeficientes relativos à matriz de massa adicional (equação 3.50), como segue-se:

$$
\begin{gathered}
X_{\dot{u}}=1,1 X_{\dot{u}} \\
Y_{\dot{v}}=1,1 Y_{\dot{v}} \\
Z_{\dot{w}}=10 Z_{\dot{w}}
\end{gathered}
$$

A figura 5.24 mostra a resposta para o caturro do sistema controlado.

Nos casos 1 e 2 observa-se que o caturro $(\theta)$ e a velocidade de submersão $(\dot{z})$ apresentam acoplamento dinâmico. O balanço $(\phi)$ e a velocidade de guinada $(\dot{\psi})$ apresentam amplitudes de zero na resposta do sistema. Estes resultados são os esperados, já que com a mudança do ângulo caturro o veículo emerge ou submerge, portanto também produz-se

\footnotetext{
${ }^{5} \mathrm{O}$ sinal de referência foi construída a partir de degraus, veja figura 5.24
} 
uma variação da posição z em relação ao tempo (figuras 5.22(d) e 5.23(d)). No caso 1 (figura 5.21(a)), o erro de tracking é igual ao caso da resposta de caturro na figura 5.20 (b). Este fato é esperado, já que os termos de ordem maior que dois, relativos ao amortecimento, foram desprezados na modelagem ${ }^{6}$ matemática do VSA. No caso 2 (figura 5.21(b)), o erro de tracking é maior quando comparado com a resposta de caturro na figura 5.20(b). Este fato também é esperado, já que o controlador $K$ foi projetado para uma velocidade de avanço muito maior $(2 \mathrm{~m} / \mathrm{s})$, o que dificulta a ação de controle a baixas velocidades $(1,1 \mathrm{~m} / \mathrm{s})$ (figura 5.23$)$.

No caso 3, o controlador opera em condições bastante adversas, mas os resultados mostraram que o veículo acompanha o sinal degrau para o caturro (figura 5.24(b)) com um ligeiro erro, o acoplamento com a velocidade de submersão é notório (figura 5.24(d)), e o acoplamento com o balanço (figura 5.24(a)) e com a velocidade de guinada (figura 5.24(c)) tiveram características parecidas a um sinal oscilante amortecido, com amplitudes pico pico menores do que $14 \times 10 e-4$.

Nos casos 1, 2 e 3 os resultados são os esperados, já que segundo a equação (4.28), $T$ pequeno garante a estabilidade robusta (ER) para incertezas da pior espécie. Porém, os picos em $S$ devem ainda ser reduzidos (equação (4.31)), já que estes picos mostram a possível degradação de desempenho do sistema. Portanto, com este controlador, existe ainda uma questão a ser resolvida relativa ao desempenho robusto (DR) do sistema, que é mais significativo para o movimento de balanço, a velocidade de guinada e a velocidade de submersão.

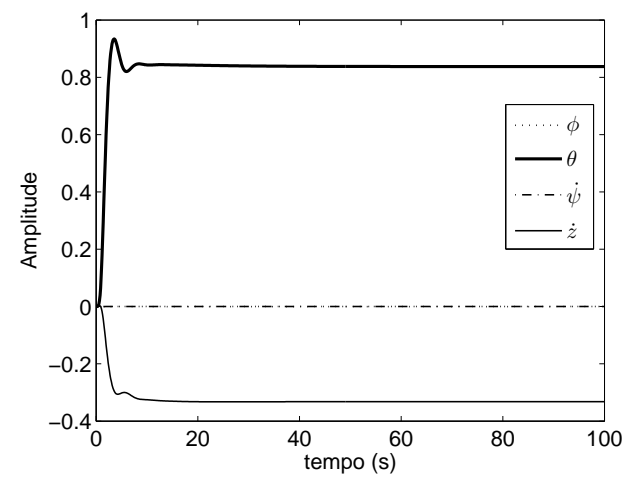

(a) Caso 1 ; condição $u=2,7 \mathrm{~m} / \mathrm{s}$

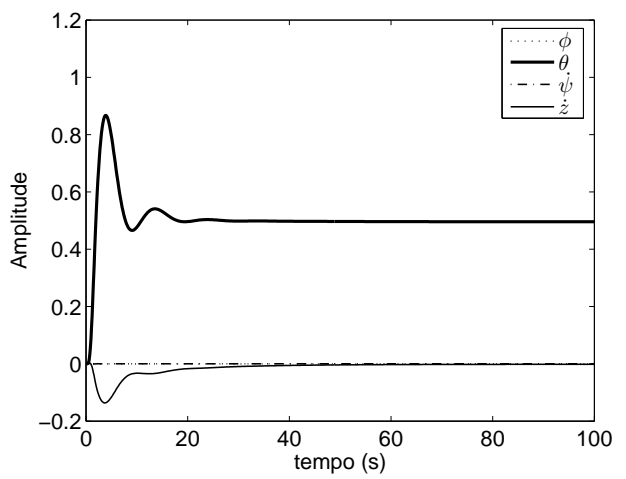

(b) Caso 2 ; condição $u=1,1 \mathrm{~m} / \mathrm{s}$

Figura 5.21: Resposta ao degrau unitário para o caturro

\footnotetext{
${ }^{6} \mathrm{~A}$ modelagem dinâmica está no capítulo 3
} 


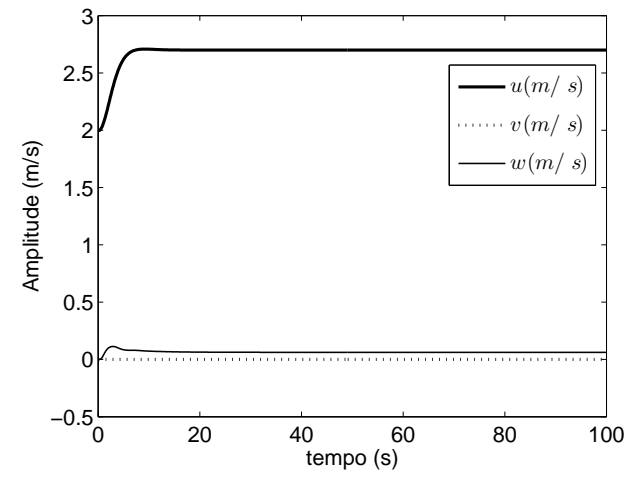

(a) veolicidade linear

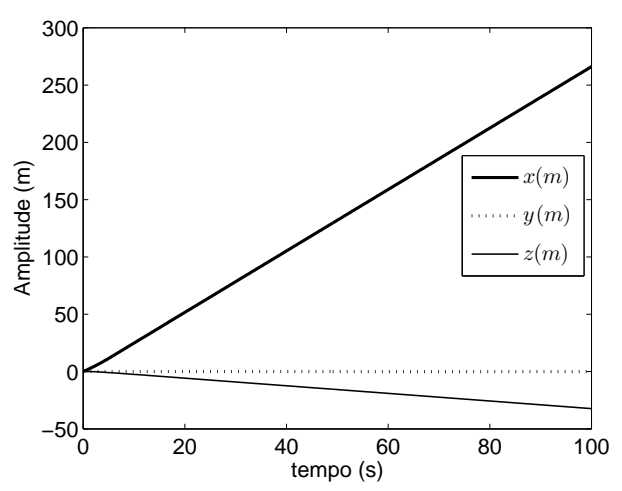

(c) posição

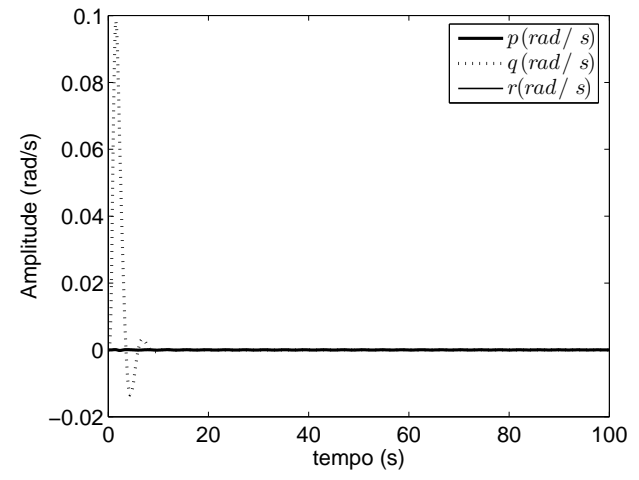

(b) velocidade rotacional

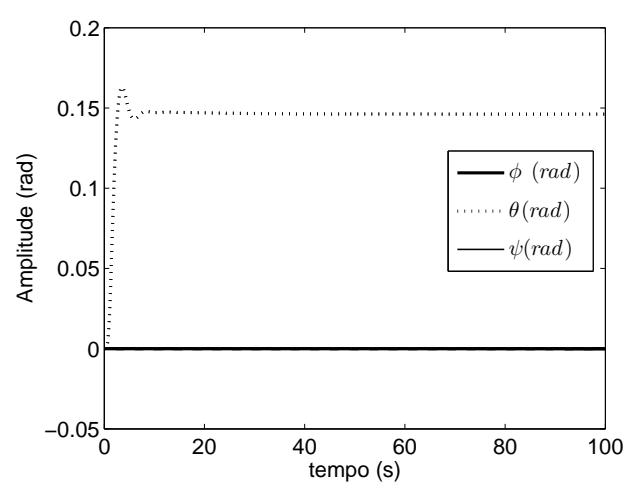

(d) atitude

Figura 5.22: Caso 1; velocidade e posição do veículo controlado (degrau unitário no caturro e velocidade de avanço de $u=2,7 \mathrm{~m} / \mathrm{s}$ ) 


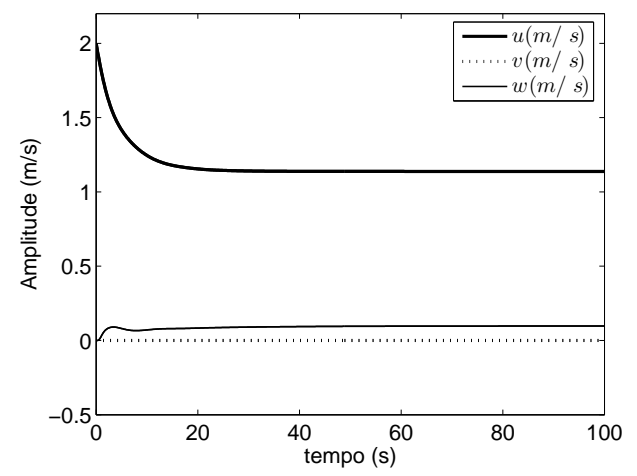

(a) velocidade linear

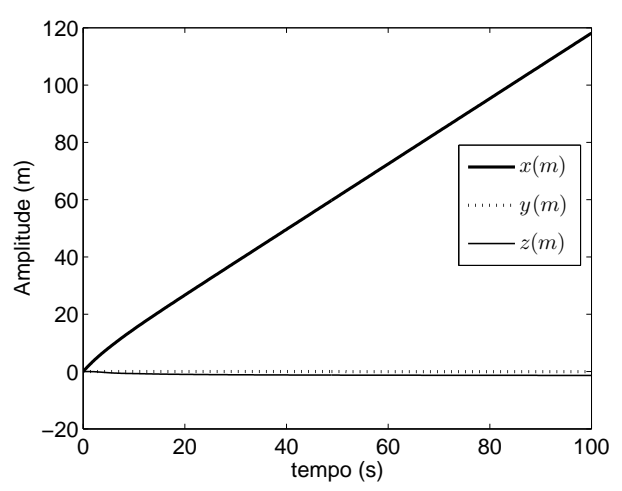

(c) posição

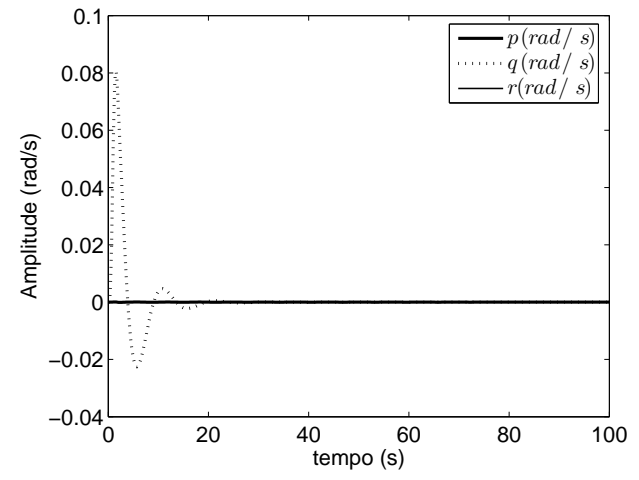

(b) velocidade rotacional

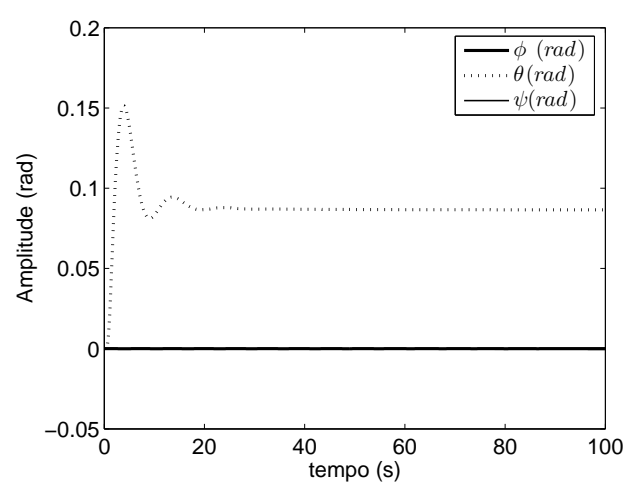

(d) atitude

Figura 5.23: Caso 2; velocidade e posição do veículo controlado (degrau unitário no caturro e velocidade de avanço de $u=1,1 \mathrm{~m} / \mathrm{s}$ ) 


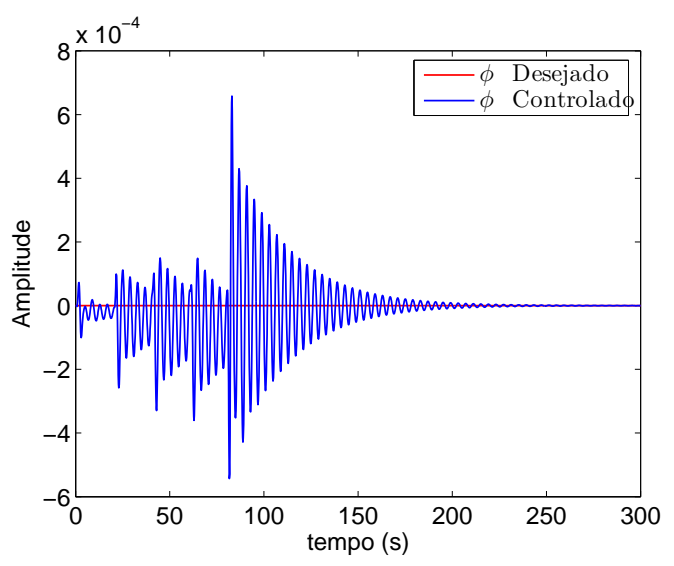

(a) balanço

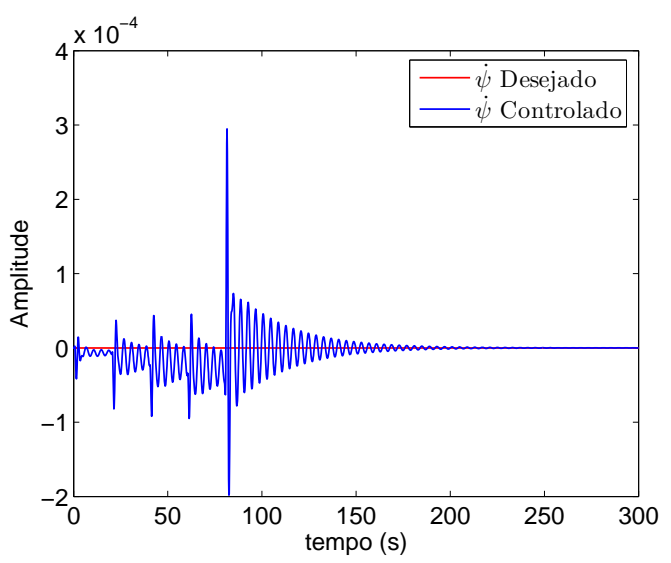

(c) velocidade de guinada

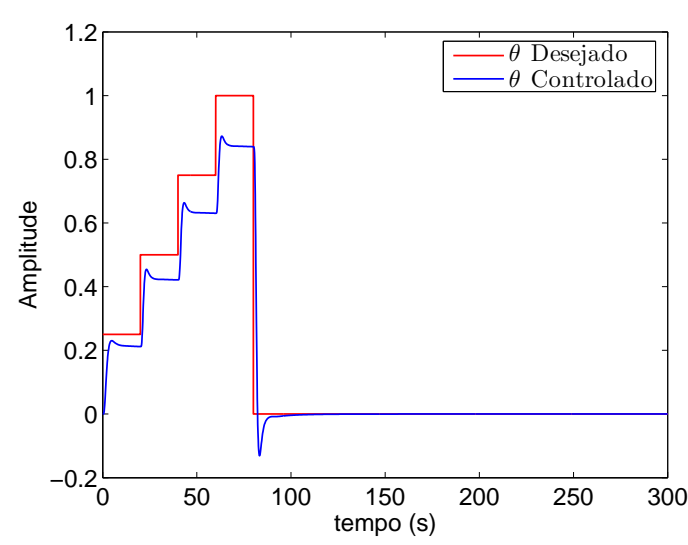

(b) caturro

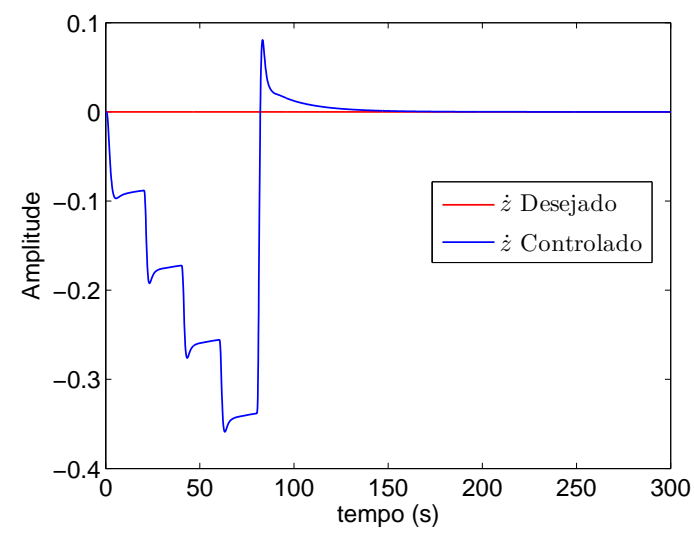

(d) velocidade de submersão

Figura 5.24: Resposta não linear do sistema controlado considerando incertezas de massa adicionada 


\subsection{Controlador de Dois Graus de Liberdade}

O excesso de robustez limita as especificações de desempenho do VSA. Os resultados obtidos usando a estrutura $\mathcal{H}_{\infty}$ de um grau de liberdade (1-GL) (ver figura 4.10), são satisfatórios do ponto de vista da estabilidade, mas ainda podem ser melhorados em relação ao desempenho (DONHA; LUQUE, 2006).

De acordo com os resultados da seção anterior, conseguiu-se ER para todos os sinais controlados, o movimento de caturro apresentou pouca degradação de desempenho, porém, o movimento de balanço, a velocidade de guinada e a velocidade de submersão tiveram um desempenho pouco satisfatório. A explicação é o fato de ter-se um sistema dinâmico sub-atuado, com muitas variáveis a serem controladas e poucos atuadores disponíveis para controlar todos esses sinais, o que torna mais exaustiva a busca de parâmetros para as funções de ponderação. Assim, recomenda-se usar uma alternativa para resolver o problema de DR.

Existem muitas alternativas para melhorar o desempenho do VSA, neste trabalho adotouse a estrutura do controlador de dois graus de liberdade (2-GL), também usada por LundstrÖm, Skogestad e Doyle (1999) (ver figura 5.25). O controlador $K$, de duas entradas y e $r$, pode ser particionado em $K=\left[\begin{array}{ll}K_{y} & K_{r}\end{array}\right]^{T}$, onde $K_{y}$ é a parte relativa à realimentação, e $K_{r}$ é a parte relativa ao préfiltro. O préfiltro $R$ é projetado de acordo com as especificações de projeto (E5). O bloco $W_{\text {model }}$ pode ser introduzido na estrutura para dar a formatação desejada do sistema em malha fechada (TANNER; GEERING, 2003).

Para a síntese do controlador 2-GL, $R$ e $W_{\text {model }}$ são matrizes identidades e as matrizes de ponderação são mantidas. Após a síntese do controlador com a nova estrutura (figura 5.25), projeta-se o préfiltro, com a seguinte estrutura:

$$
R=\left[\begin{array}{cccc}
\frac{1.76}{s+0.2} & 0 & 0 & 0 \\
0 & \frac{0.6}{s+0.2} & 0 & 0 \\
0 & 0 & \frac{9.8}{s+0.2} & 0 \\
0 & 0 & 0 & \frac{-10}{s+0.05}
\end{array}\right]
$$

A figura 5.26 mostra a resposta temporal do sistema, onde o controlador de 2-GL foi utilizado. O desempenho melhorou, mas viu-se comprometida a estabilidade relativa ao balanço e à guinada. Todos os sinais controlados acompanham os sinais de referência.

A resposta para a velocidade de guinada 5.26(c) é oscilante, isto deve-se ao fato de que a alta velocidade de cruzeiro limita a manobrabilidade do VSA. As oscilações na resposta para o balanço (figura 5.26(a)) também têm a mesma interpretação, já que de acordo a figura 5.7(d) este movimento é fortemente acoplado com a velocidade de guinada. 
A resposta para o caturro (figura 5.26(b)) é lisa e bem estável, não há acoplamento com o movimento de balanço, nem com a velocidade de guinada.

A resposta para a velocidade de submersão (figura 5.26(d)) também é lisa, neste caso, os acoplamentos com o balanço e a velocidade de guinada são mínimos.

A Principal causa das oscilações na resposta do sistema é o fato do VSA ser sub-atuado, e as limitações físicas da própria dinâmica do veículo. Assim projeta-se um limitador para todos os sinais de entrada no sistema controlado com valores extremos entre $-0,5$ e 0,5 . A figura 5.27 mostra a resposta ao degrau 0,5 do sistema, as oscilações de balanço e velocidade de guinada desaparecem completamente no regime permanente. Estabilidade e Desempenho Robustos foram finalmente alcançados.

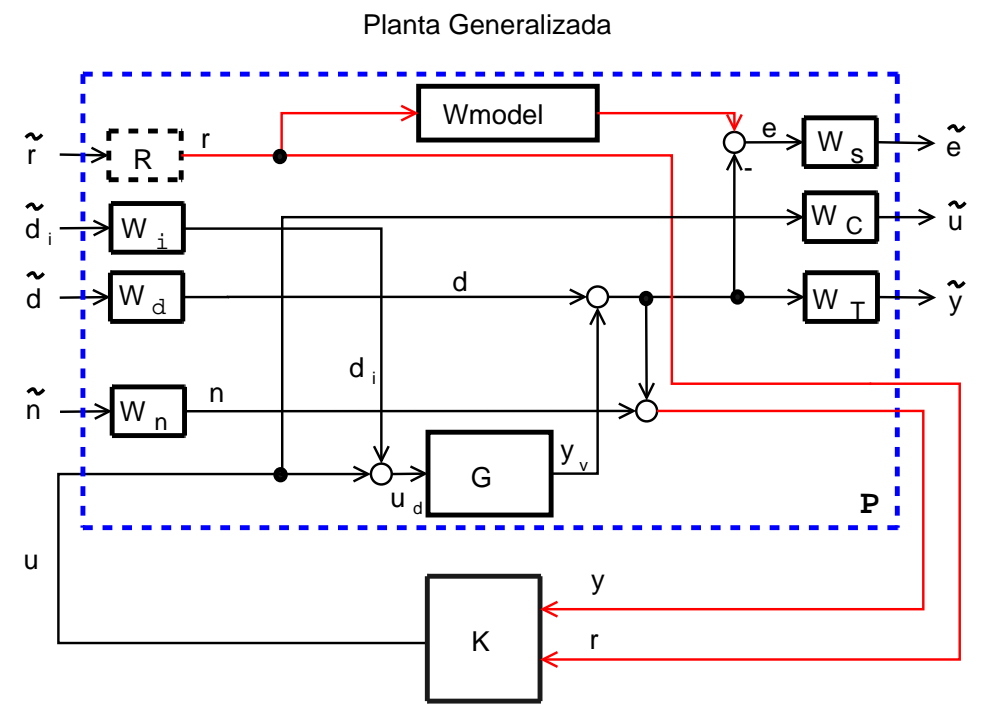

Figura 5.25: Controlador de dois graus de liberdade, configuração genérica de dois portos. 
(a)

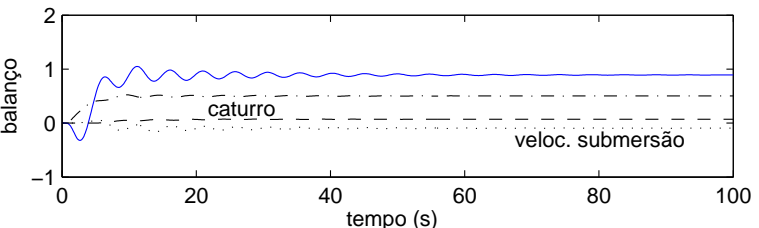

(b)

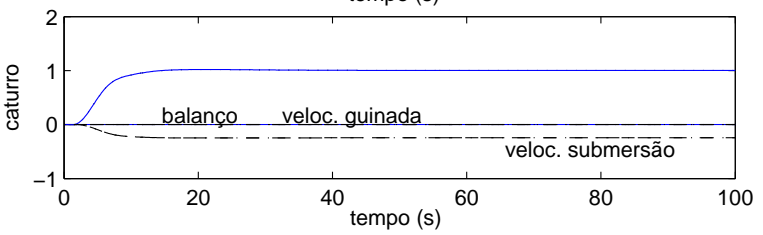

(c)

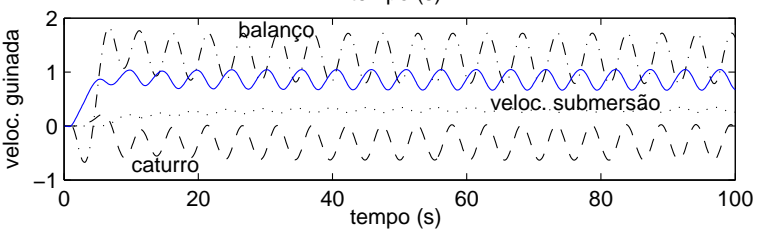

(d)

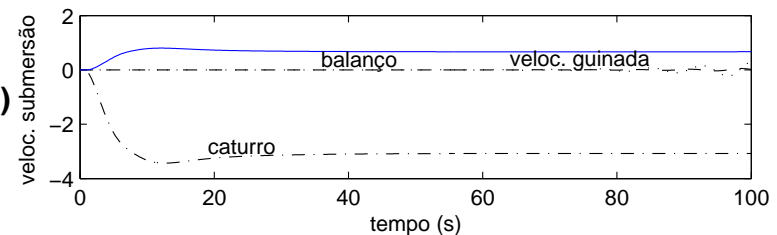

Figura 5.26: Resposta ao degrau unitário com o controlador de 2-GL - caso não linear.
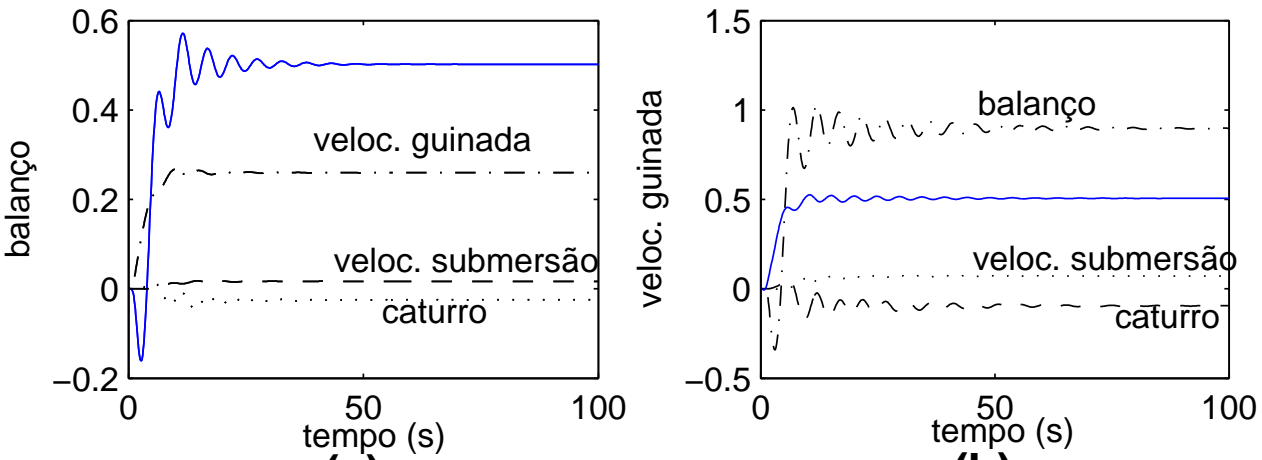

(a)

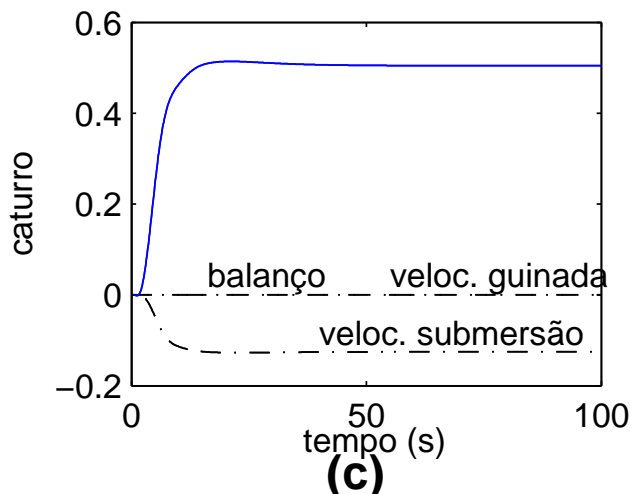

(b)

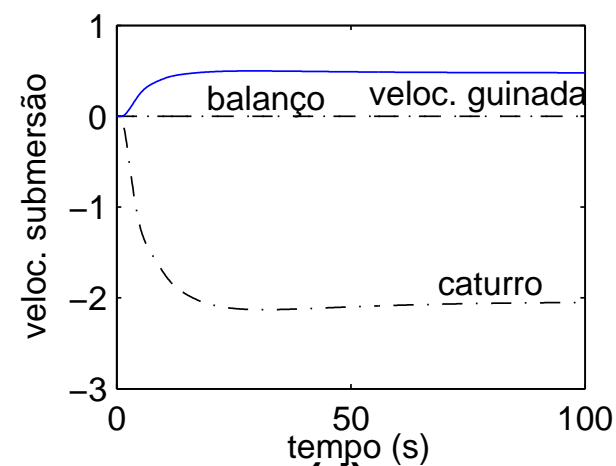

(d)

Figura 5.27: Resposta ao degrau 0,5 com o controlador de 2-GL - caso não linear. 


\subsection{Guiagem}

Com os resultados anteriores satisfatórios, parte-se agora para a implementação do sistema de guiagem, procurando testar o veículo na realização de missões de exploração envolvendo o acompanhamento de trajetórias espaciais.

O sistema de guiagem é encarregado de guiar o veículo até um alvo ou acompanhar uma trajetória desejada, utilizando para este propósito a leitura das variáveis $(x, y$ e $z) \mathrm{e}$ transformações cinemáticas, gerando o sinal de comando para o sistema propulsor. O Controlador Robusto aciona as superfícies de controle (lemes) a partir dos sinais observados do sistema. A velocidade de avanço deve permanecer constante $(2 \mathrm{~m} / \mathrm{s})$ em todo o trajeto.

A figura 5.28 mostra a inclusão do bloco de guiagem na arquitetura de controle geral do sistema. A missão do veículo agora é acompanhar uma determinada trajetória, construída a partir de alvos desejados representados pela triade $\left[x_{d}(k), y_{d}(k), z_{d}(k)\right]$, onde $k=1,2, \ldots, N$ define a linha de visão ou LOS (Line of Sight) para a guinada (HEALEY; LIENARD, 1993).

Neste trabalho, usa-se uma forma modificada da LOS para a velocidade de guinada, onde:

$$
\begin{gathered}
\psi_{d}(t)=\tan ^{-1}\left(\frac{y_{d}(k)-y(t)}{x_{d}(k)-x(t)}\right) \\
\dot{\psi}_{d}(t)=\frac{d}{d t}\left\{\psi_{d}(t)\right\}
\end{gathered}
$$

A velocidade de guinada $\left(\dot{\psi}_{d}\right)$, que entra no bloco do controlador realiza a guiagem do VSA no plano horizontal. Para avaliar a guiagem, são geradas duas trajetórias básicas para o VSA: o círculo e o zig-zag.

A figura 5.30 mostra a guiagem do VSA numa trajetória circular. A figura 5.31 mostra a movimentação dos lemes nesta tarefa. Na figura 5.32 observa-se o sinal de comando controlado (velocidade de guinada), onde nota-se que o sinal é bastante liso, indicando boa rejeição de distúrbios. A posição do veículo relativamente ao referencial fixo encontra-se na figura 5.34, onde observa-se que apesar do forte acoplamento verificado anteriormente, o VSA mantém a trajetória horizontal plana e chega à trajetória assintoticamente, o que pode ser melhor apreciado na figura 5.30.

Como o VSA é sub-atuado, quando o veículo acompanha a trajetória circular, ocorre o balanço e a variação da velocidade de guinada. Porém, o ângulo de caturro, com magnitude de 0,15 , mantém a posição $z=0$ (ver figura 5.34) indicando que houve compensação dos esforços de sustentação gerados pela velocidade de avanço $u=2 \mathrm{~m} / \mathrm{s}$. 


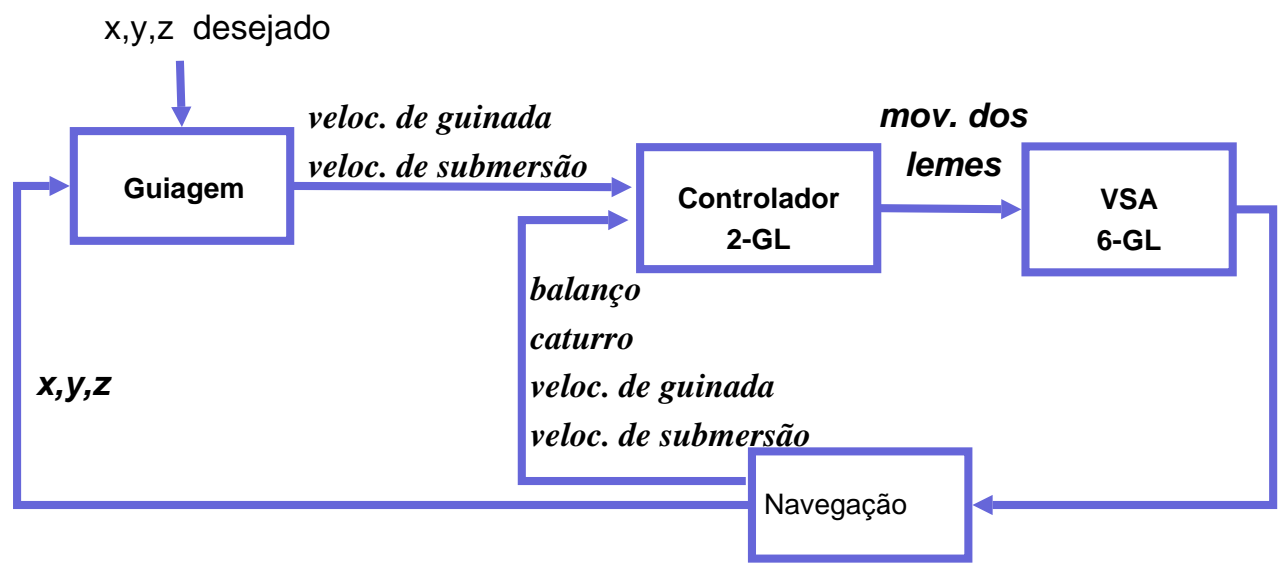

Figura 5.28: Diagrama de Blocos: Guiagem e Controle para o VSA.

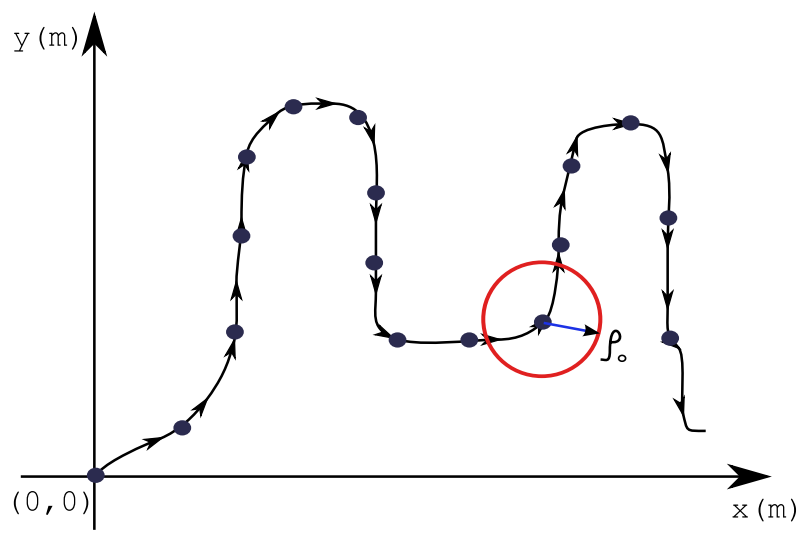

Figura 5.29: Trajetória desejada de acordo com LOS: Os alvos são representados por pequenos círculos, e o círculo maior representa a restrição imposta $\left(\right.$ raio $\left.=\rho_{o}\right)$.

A figura 5.35 mostra a guiagem numa trajetória zig-zag, onde observa-se um pequeno erro de acompanhamento (offset) considerando-se a alta velocidade de cruzeiro. A figura 5.36 mostra a movimentação dos lemes nesta tarefa. Na figura 5.37 observa-se o sinal de comando (velocidade de guinada). A posição do veículo relativamente ao referencial fixo encontra-se na figura 5.39.

Quando o veículo acompanha a trajetória zig-zag, o acoplamento balanço/ velocidade de guinada é claro (figura 5.38), mas o valor do ângulo caturro é pequeno.

O sistema de guiagem para LOS mostrou o bom acompanhamento das trajetórias desejadas. A flexibilidade da lei de Controle Robusta e o sistema de guiagem, desenvolvidos para o veículo submersível autônomo, permitem acompanhar trajetórias diversas e complexas, como a trajetória helicoidal ilustrada na figura 5.40. 


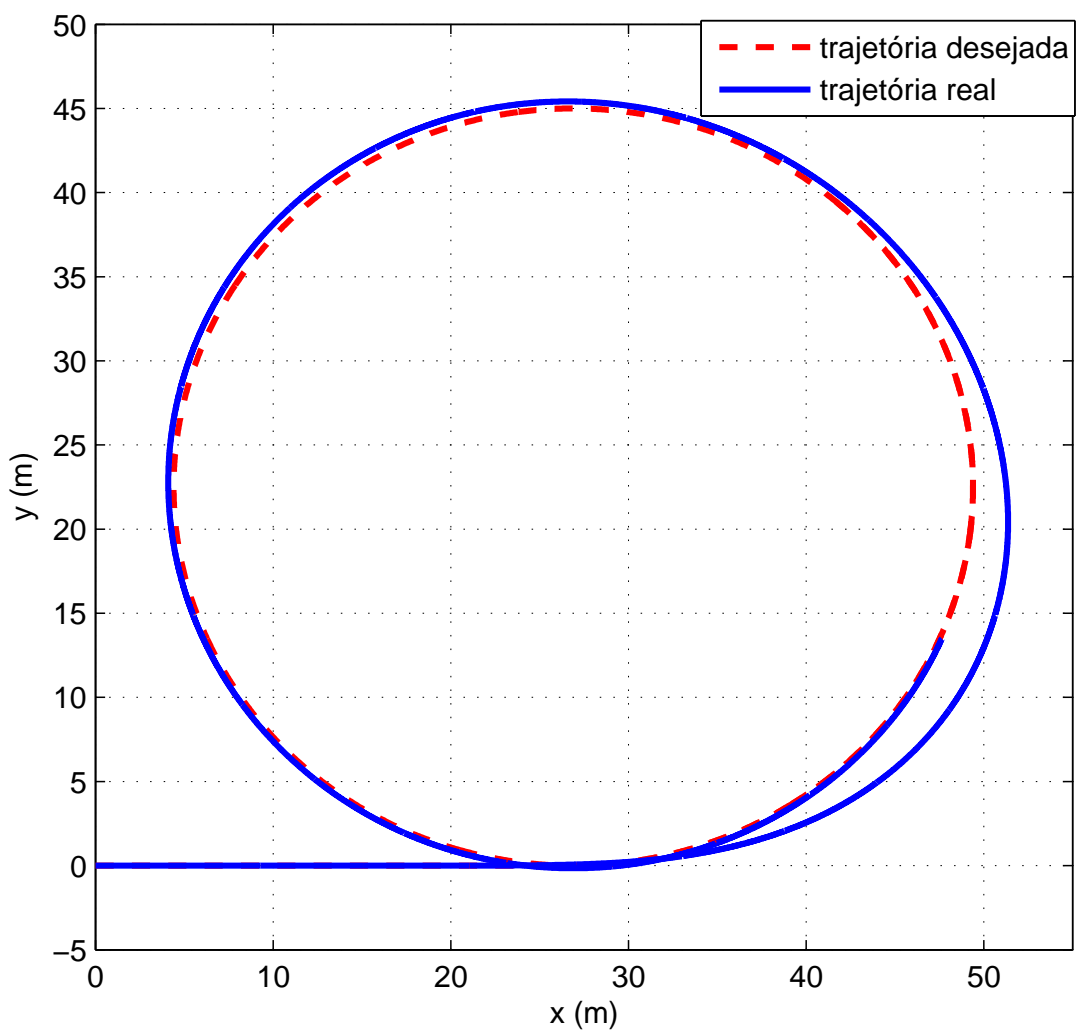

Figura 5.30: Trajetória circular: $u=2 \mathrm{~m} / \mathrm{s}$.

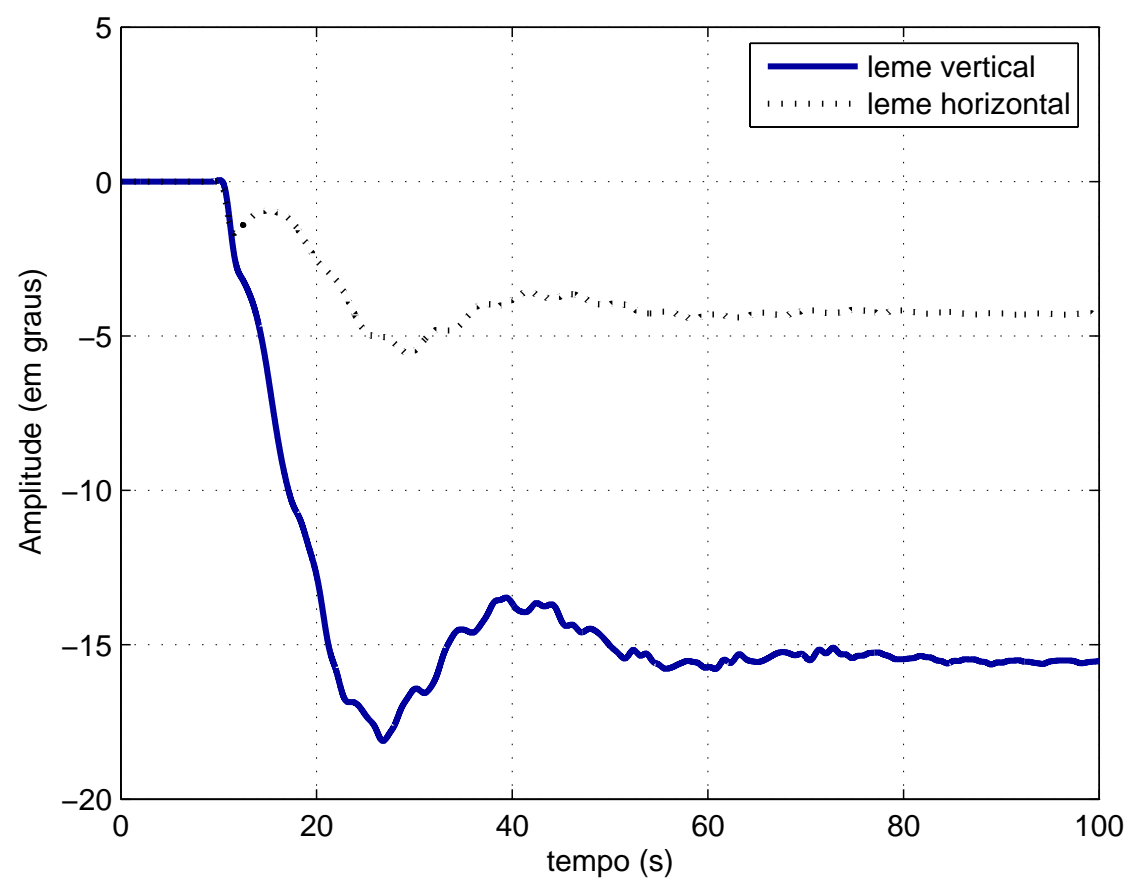

Figura 5.31: Trajetória circular: movimento dos lemes. 


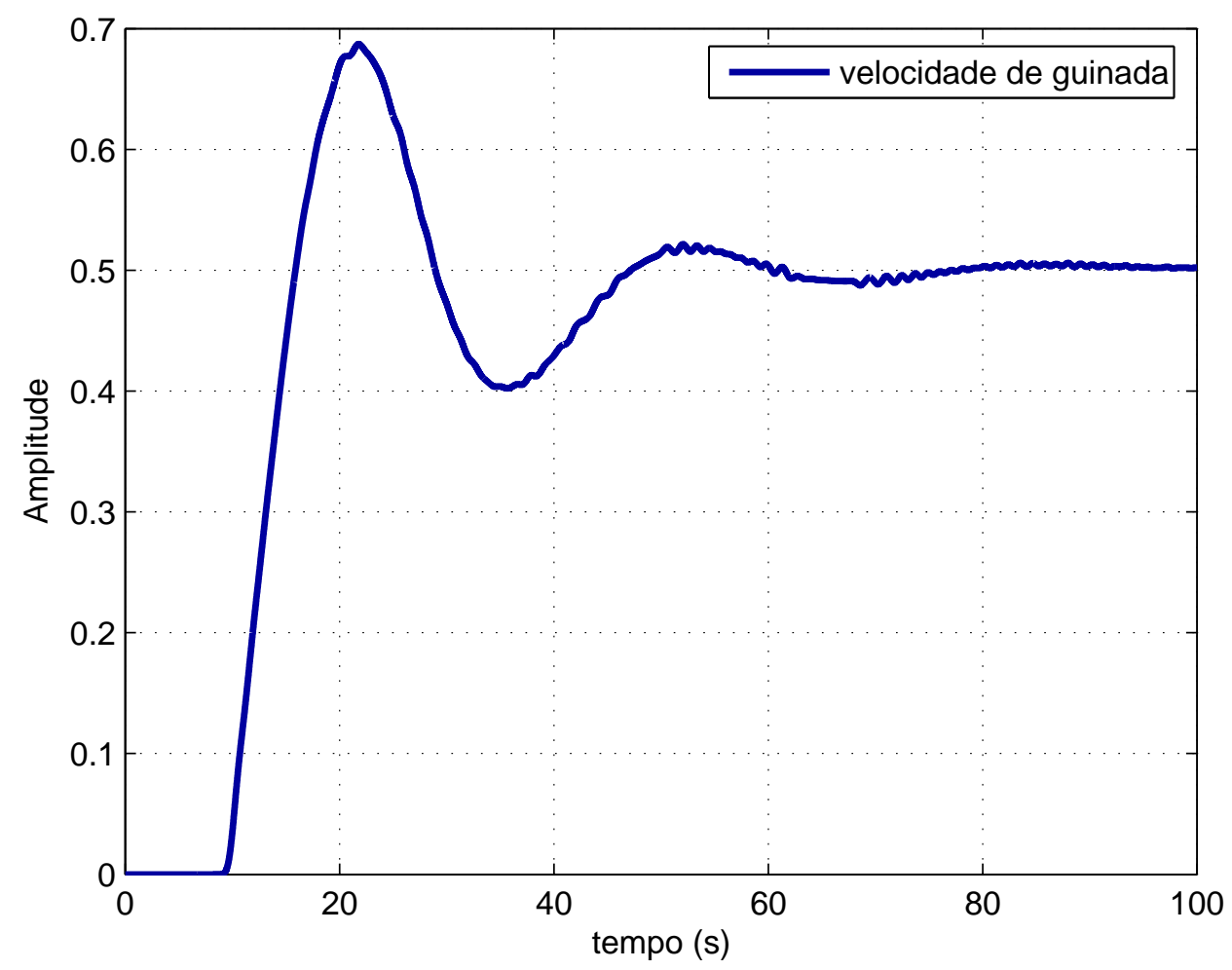

Figura 5.32: Trajetória Circular: sinal de comando.

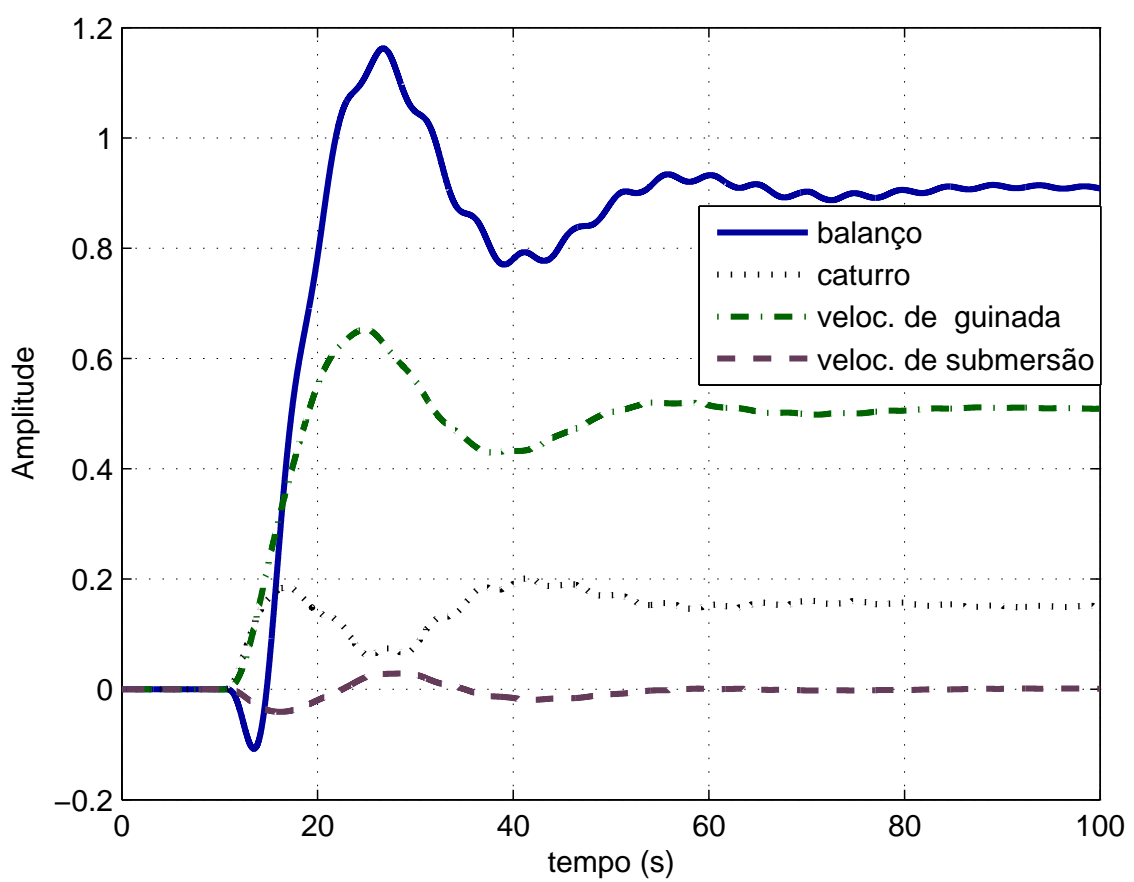

Figura 5.33: Trajetória circular: resposta do sistema controlado. 


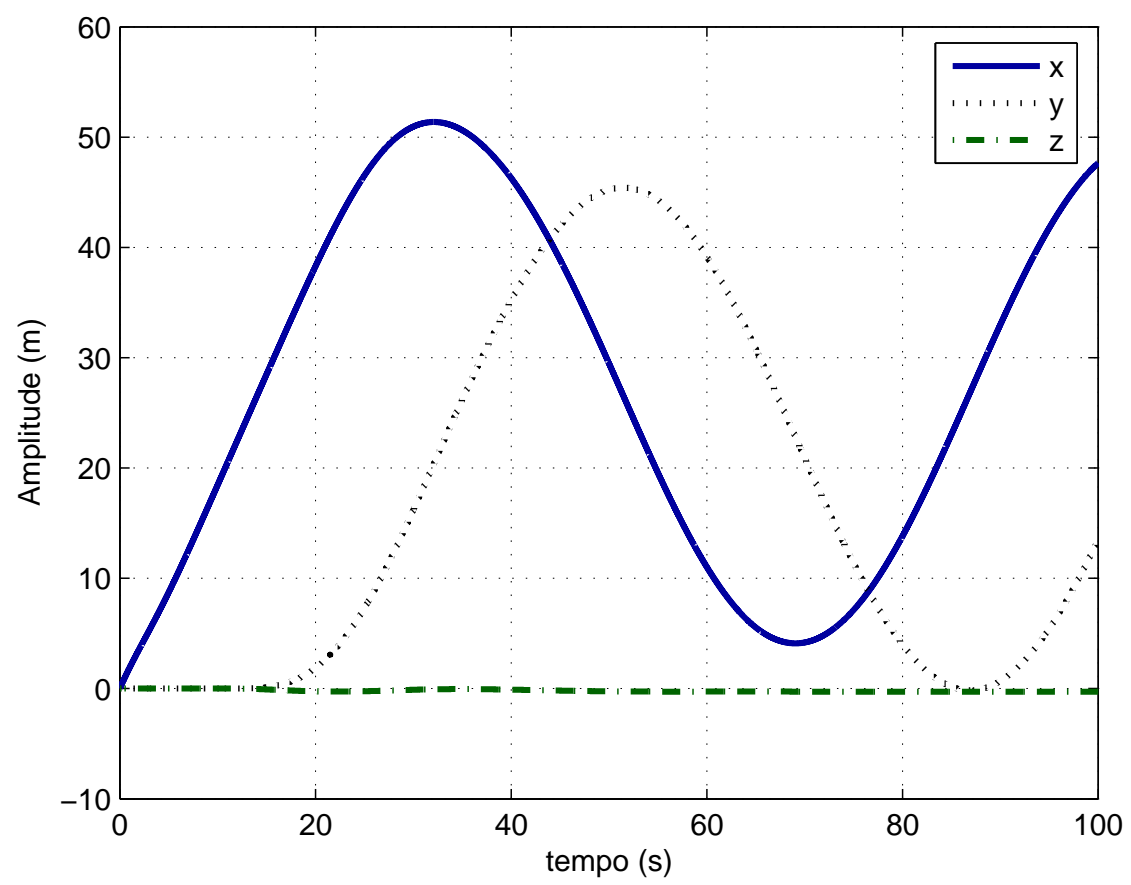

Figura 5.34: Trajetória Circular, posição $x, y$ e $z$ do VSA.

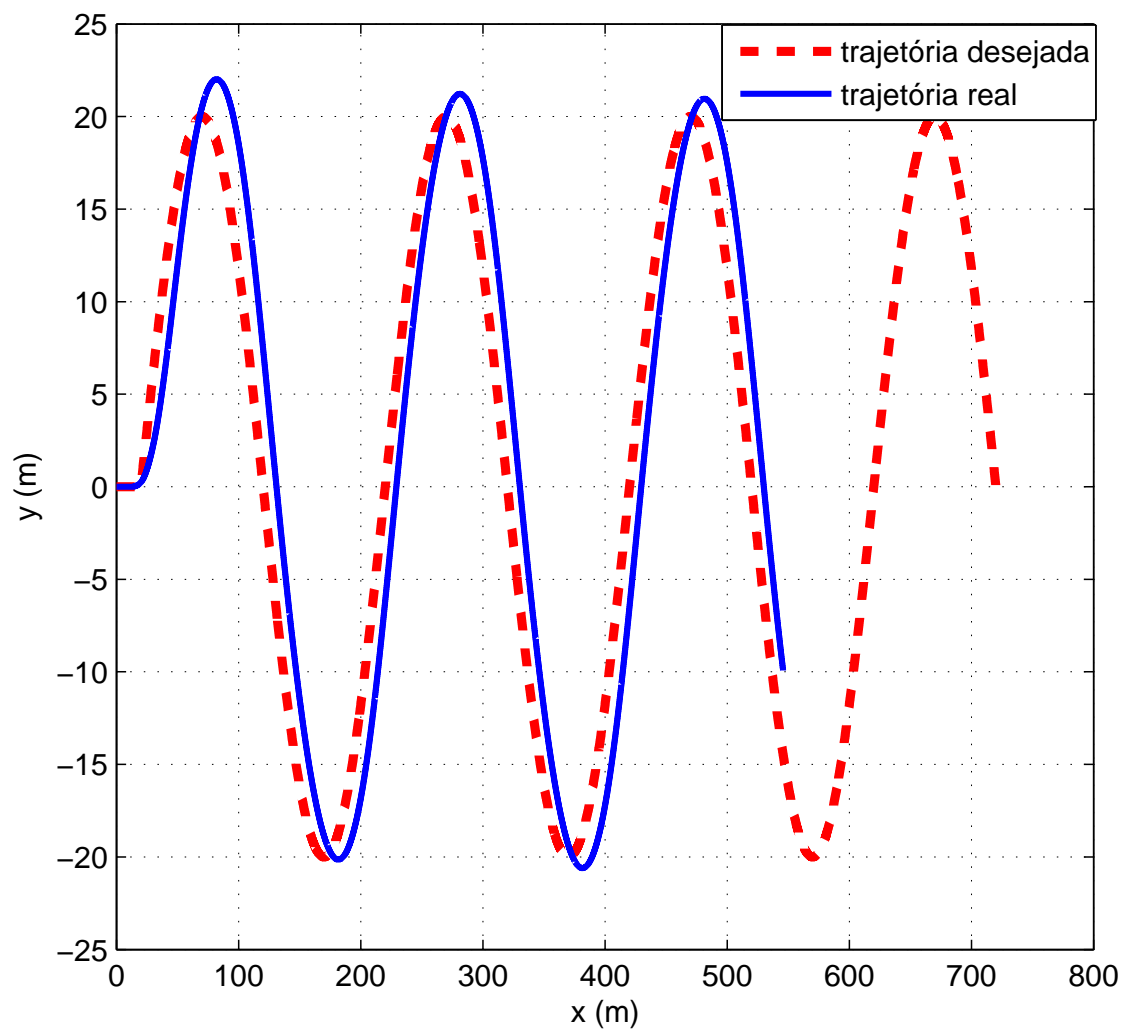

Figura 5.35: Trajetória zig-zag: $u=2 \mathrm{~m} / \mathrm{s}$. 


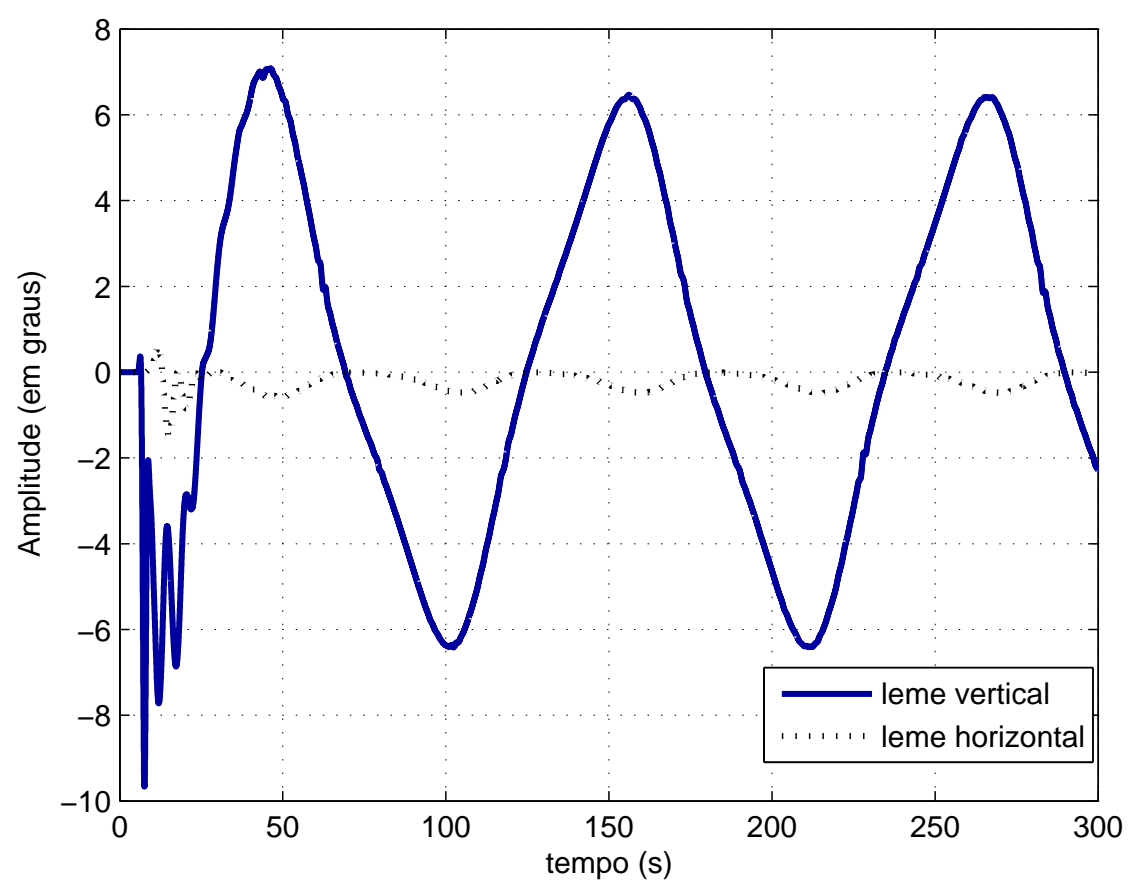

Figura 5.36: Trajetória zig-zag: movimento dos lemes.

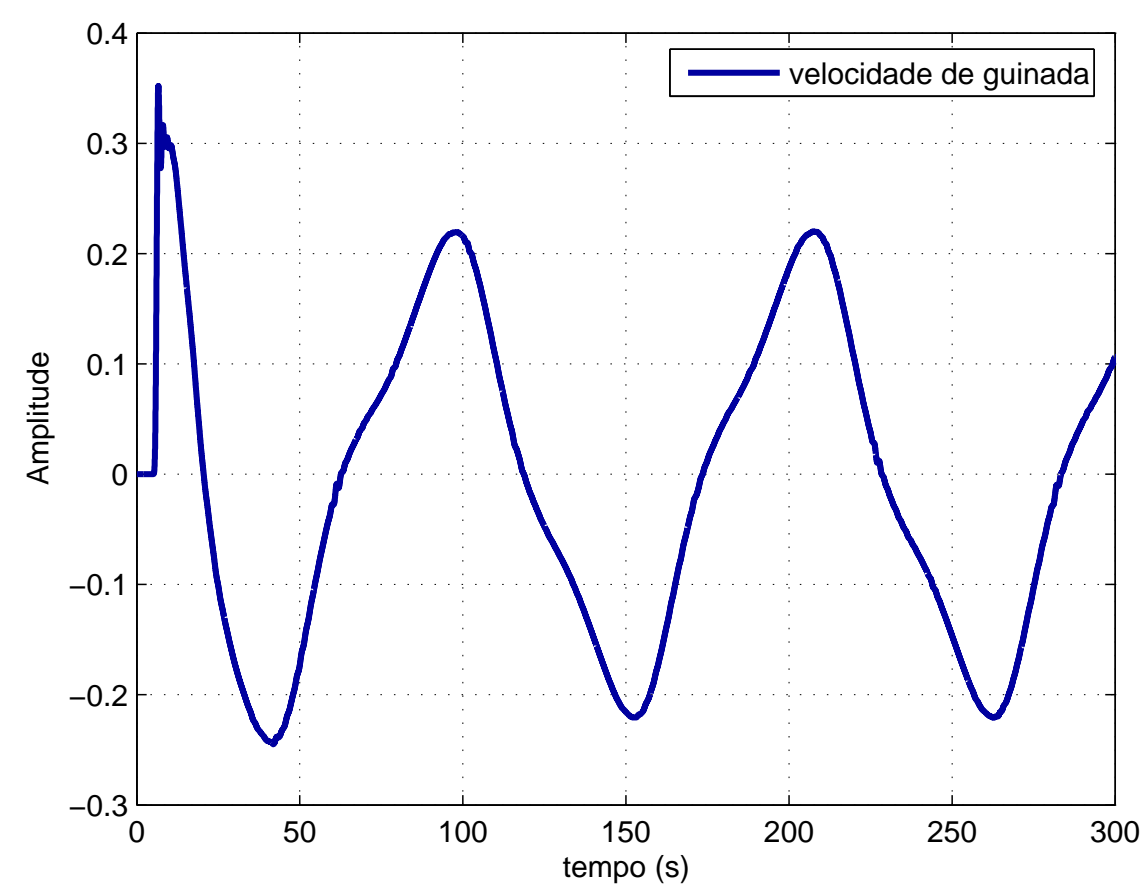

Figura 5.37: Trajetória zig-zag: sinal de comando. 


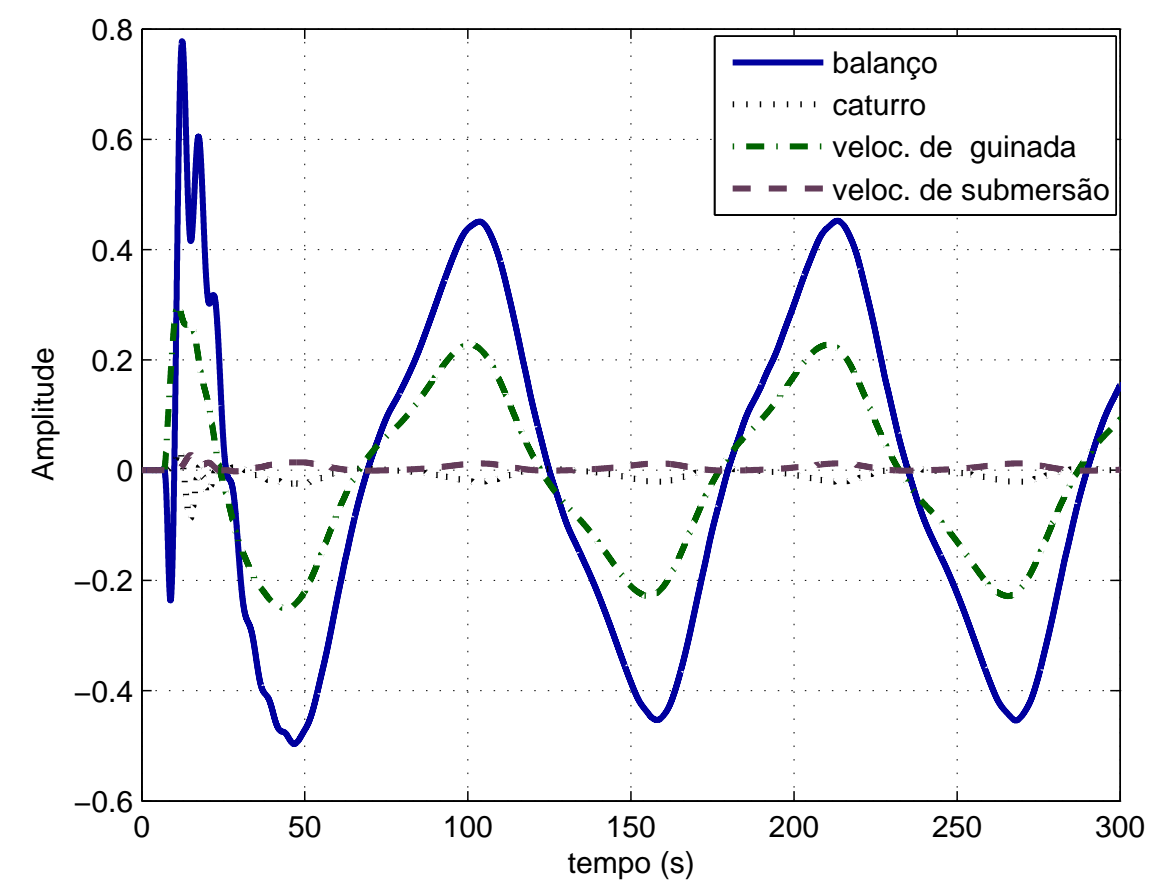

Figura 5.38: Trajetória zig-zag: resposta do sistema controlado.

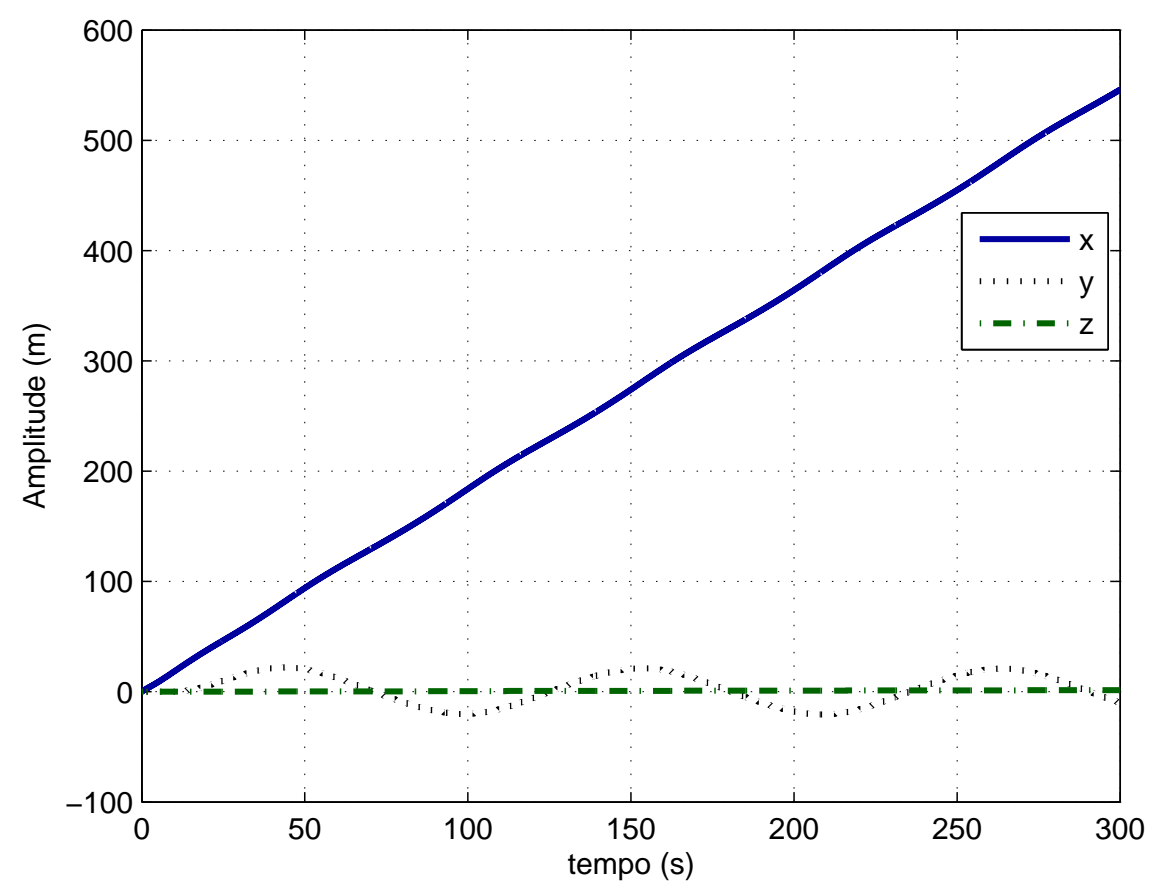

Figura 5.39: Trajetória zig-zag: posição $x, y$ e $z$ do VSA. 


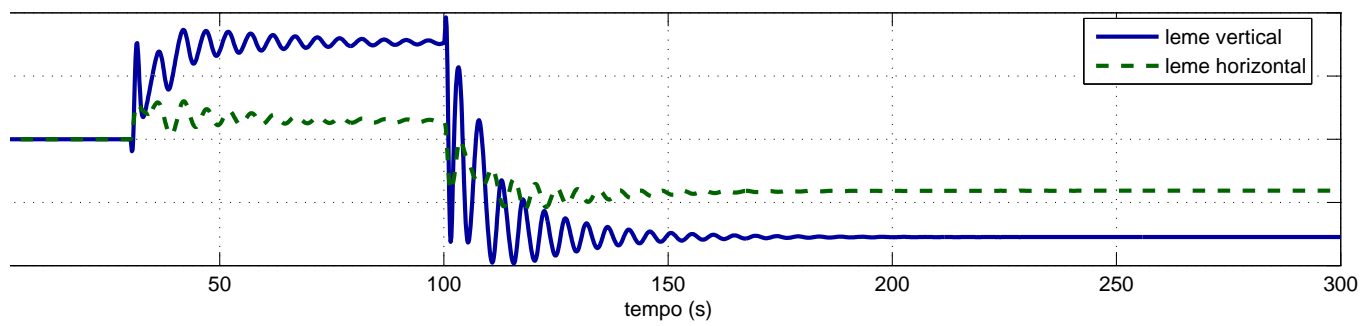

(a) Movimento dos lemes

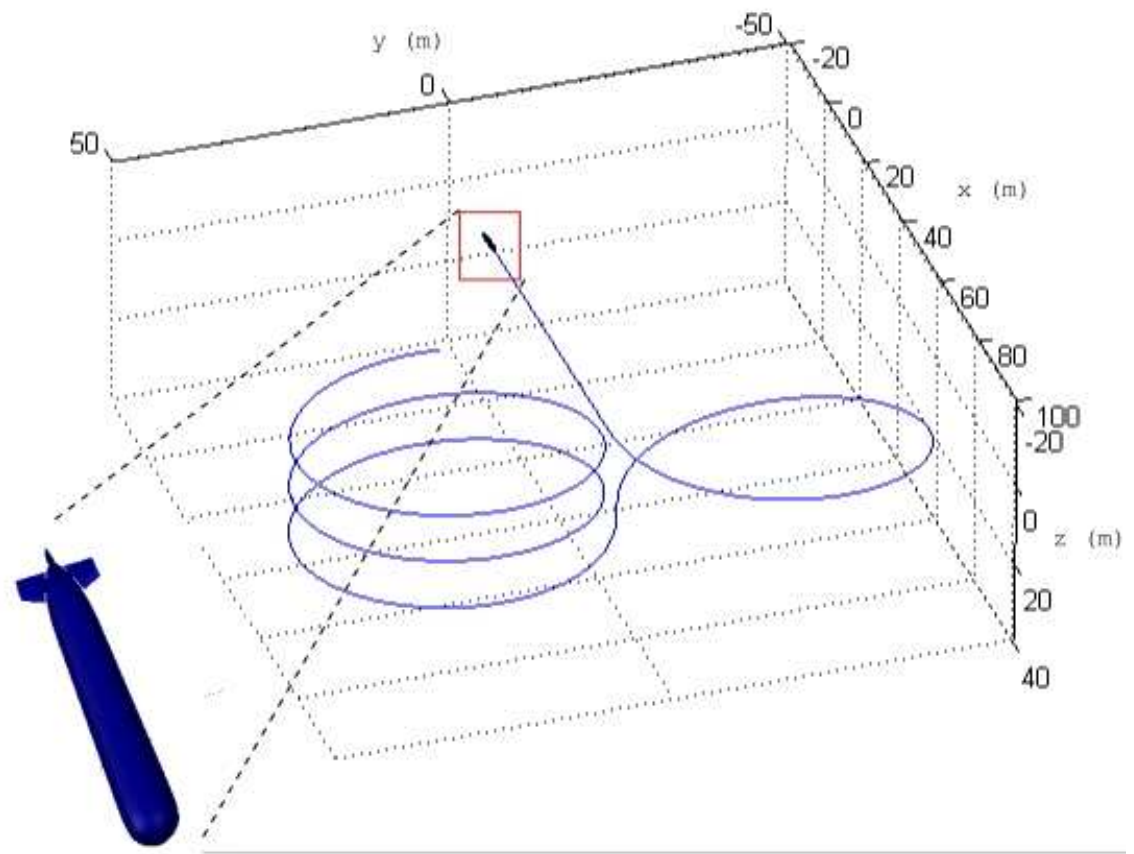

(b) Posição do veículo

Figura 5.40: Trajetória helicoidal do VSA 


\section{Conclusões}

Neste trabalho inicialmente escolheu-se e refinou-se um modelo matemático não linear para um Veículo Submersível Autônomo .Após extensa pesquisa e validações chegou-se a um modelo não linear completo com seis graus de liberdade (6-GL) expresso for equações diferenciais ordinárias a parâmetros constantes. O modelo não linear de 6-GL foi validado através de simulações numéricas. Em seguida, foram obtidos modelos lineares em pontos distintos de operação. A análise dos resultados permitiu identificar o forte acoplamento dinâmico existente entre suas variáveis. Finalmente, como fóco principal nesta pesquisa, projetou-se, validou-se e testou-se a Lei de Controle Robusto $\mathcal{H}_{\infty}$ para controlar de maneira centralizada todos os movimentos do veículo submersível.

A modelagem e a técnica de controle robusto demandaram muito esforço. O trabalho realizado mostrou que é possível controlar o sistema, apesar das incertezas com relação à dinâmica do veículo, aos distúrbios e ruídos de natureza diversa. O controlador robusto multivariável é capaz de manter estabilidade robusta e especificações de desempenho são garantidas.

A técnica de Sensibilidade Mista foi utilizada para sintetizar o controlador, capaz de atender às especificações de estabilidade e desempenho robustos para o projeto do controlador. Em seguida, foram analisadas todas as respostas relativas à sensibilidade do sistema até encontrarem-se os parâmetros que satisfizessem as especificações de maneira ótima. Certamente, o maior esforço de síntese esteve associado à busca dos parâmetros das funções de ponderação que resolvem o problema de controle para o VSA.

A resposta do sistema controlado foi avaliada no domínio do tempo, tanto para o caso linear quanto para o caso não linear, onde verificou-se novamente a estabilidade robusta. $\mathrm{O}$ veículo também foi controlado ainda em pontos de operação muito diferentes, onde incertezas de modelagem foram consideradas nos coeficientes de massa adicional, verificando-se que a robustez do controlador, conseguindo-se estabilidade robusta no sistema, embora o desempenho tenha-se degradado ligeiramente.

A técnica de controle robusto aplicada no veículo submersível autônomo assume o tipo de incertezas não estruturadas, portanto, pode ser demasiado conservadora em relação 
à estabilidade e algumas especificações do desempenho não podem ser alcançadas. Este problema ainda é exacerbado, já que o VSA aqui estudado é sub-atuado. A estrutura do controlador 2-GL foi então adotada para a solução deste problema, através da introdução de um filtro na entrada do sistema. Assim, os resultados finais no domínio do tempo mostraram-se completamente satisfatórios com o uso da técnica de Controle Robusto $\mathcal{H}_{\infty}$ por Sensibilidade Mista.

Um sistema de guiagem foi elaborado para o controle do VSA, buscando emular a realização de missões típicas e verificar a capacidade de acompanhamento de trajetórias pré-programadas ou desejadas. Os resultados alcançados foram também muito satisfatórios e o VSA controlado pode acompanhar até trajetórias tridimensionais.

Finalmente, o controlador robusto multivariável desenvolvido neste trabalho mostrou boas características de estabilidade e desempenho robustos, permitindo o controle de todos os movimentos do VSA de maneira centralizada.

\subsection{Sugestões para trabalhos futuros}

A seguir, apresentam-se algumas sugestões e trabalhos futuros a serem encaminhados a partir deste trabalho.

Sugere-se, inicialmente, o modelamento de correntezas marinhas, e a verificação da robustez e do desempenho do VSA de forma mais rigorosa nessa nova situação, o que pode eventualmente levar a modificações dos parâmetros das funções de ponderação. Recomenda-se modelar os distúrbios presentes no meio submarino, e aproximá-los por modelos lineares, o que pode facilitar a busca de parâmetros para as funções de ponderação.

Para sistemas multivariáveis e sub-atuados, a normalização converte-se num fator fundamental na síntese de um controlador robusto centralizado, pois facilita a busca de parâmetros das funções de ponderação. Recomenda-se, então, extrema atenção nesta fase do projeto. Outro fator importante é relativo às incertezas do sistema. Usando-se incertezas estruturadas pode-se aliviar o problema do excesso de conservadorismo relativo às incertezas da pior espécie $\left(\|\Delta\|_{\infty}=1\right)$. Com a síntese- $\mu$ deve ser possível melhorar o desempenho do controlador. A mesma estrutura do controlador 2-GL usada neste trabalho pode também ser usada na síntese do controlador usando a técnica de síntese- $\mu$ (LUNDSTRÖM; SKOGESTAD; DOYLE, 1999).

Podem-se também fazer comparações com outras técnicas de controle como o controle PID, o Controle Difuso, o Controle $\mathcal{H}_{2}$, o Controle $L Q G / L T R$, o Controle por Modos 
Deslizantes, o Controle Adaptativo, etc.

Pode-se otimizar o controlador 2-GL desenvolvido neste trabalho, realizando uma comparação entre as abordagens de malha aberta e de malha fechada, onde as especificações do projeto e o número de parâmetros envolvidos serão avaliados como sugerido em Tanner e Geering (2003).

Na parte relativa ao sistema de guiagem de submersíveis, devido à forma hidrodinâmica do torpedo, $x, y$ e $z$ são variáveis não controláveis, portanto, sugere-se a implementação do sistema de guiagem para o VSA. O sistema de guiagem proposto neste trabalho pode ser comparado com outras técnicas de guiagem, como no trabalho de Ryoo, Shin e Tahk (2005), onde os autores utilizam uma otimização para efetuar o acompanhamento de trajetórias. Também pode-se usar lógica difusa para comandar o sistema de guiagem LOS (LIN; MON, 2001).

Em Geral, técnicas de controle avançados como LQG e $\mathcal{H}_{\infty}$ produzem controladores de ordem elevada, portanto, exigem demasiados requisitos para a implementação em hardware. Sugere-se a utilização de técnicas de redução de ordem para o controlador, como truncamento balanceado, balanço residual ou aproximação da norma Hankel (SKOGESTAD; POSTLETHWAITE, 1996). 


\section{Referências}

ABKOWITZ, M. A. Stability and Motion Control of Ocean Vehicles. Cambridge, Massachusetts and London, England: The M.I.T. PRESS, 1969.

AVILA, J. J. Estimação de Coeficientes Hidrodinâmicos de um Veículo Submarino Semi-Autônomo. 128 p. Dissertação (Mestrado) — Escola Politécnica, Universidade de São Paulo, São Paulo, 2003.

BARROS, E. de; PASCOAL, A.; SA, E. de. Auv dynamics: modelling and parameter estimation using analytical, semi-empirical, and CFD methods. In: CAMS'04. Proceedings of the IFAC Conference on Control Applications in Marine Systems. Ancona, Italy: Dipartimento di Ingegneria Informatica, e dell'Automazione, Università Politécnica delle Marche, 2004. p. 369-376.

BO, H. Hydrodynamic Estimation and Identification. Dissertação (Master's Thesis) Department of Engineering Cybernetics, Trondheim, Norway and Instituto Superior Técnico, ISR, Lisbon, Portugal, 2004.

CANHETTI, A. C. Modelo Livre de Submarinos. 147 p. Dissertação (Mestrado) Escola Politécnica, Universidade de São Paulo, São Paulo, 1998.

CRUZ, J. J. da. Controle Robusto Multivariável. São Paulo, Brasil: EDUSP, 1996.

DOMICINIANO, V. Integração Casco-Motor-Hélice do Modelo-Livre - S3201-8420RG-01. Brasil, 1996. Centro Tecnológico da Marinha em São Paulo, São Paulo, 1995.

DONHA, D. C. Projeto de Sistemas de Controle Robustos Multivariáveis, Apostila. São Paulo, Brasil: Poli-USP, 2003.

DONHA, D. C.; KATEBI, R. M. Automatic cost weigth selection in mixed sensitivity controller synthesis. In: CAMS'04. Proceedings of the IFAC Conference on Control Applications in Marine Systems. Ancona, Italy: Dipartimento di Ingegneria Informatica, e dell'Automazione, Università Politécnica delle Marche, 2004. p. 279-284.

DONHA, D. C.; LUQUE, J. C. C. Six dof robust control. In: Proceedings of the International Control Conference. Glasgow, Scotland: University of Strathclyde, 2006.

DOYLE, J.; FRANCIS, B.; TANNENBAUM, A. Feedback Control Theory. Englewood Cliffs, NJ: Macmillan Publishing Co, 1990.

FELDMAN, J. Revised Standard Submarine Equations of Motion. Bethesda, Maryland 20084: David W. Taylor Naval Ship Research and Development Center, 1979.

FOSSEN, T. I. Guidance and Control of Ocean Vehicles. West Sussex PO19 1UD, England: John Whiley \& Sons, 1994. 
FRANCIS, B. A. A course in $\mathcal{H}_{\infty}$ Control Theory. Toronto, Canada: Springer-Verlag, 1988.

HEALEY, A. J.; LIENARD, D. Multivariable sliding model control for unmanned underwater vehicles. IEEE Journal of Oceanic Engineering, v. 18, n 3, p. 327-339, 1993.

HOERNER, S. F. Fluid Dynamics Drag. PO Box 65283, Vancouver, WA 98665: now published by the Author's estate, 1965. ISBN 9993623938.

HOERNER, S. F. Fluid Dynamics Lift. 2nd ed. PO Box 65283, Vancouver, WA 98665: now published by the Author's estate, 1992. ISBN 9998831636.

HOVER, F. S. Physical modelling of the odyssey II Vehicle. MIT Sea Grant / WHOI: The M.I.T. Press, 1995.

HUGHES, P. C. Spacecraft Attitude Hydrodynamics. New York, USA: John Whiley \& Sons, 1986.

IMLAY, F. H. The Complete Expressions for Added Mass of a Rigid Body Moving in a Ideal Fluid. Washington D.C.: David Taylor Model Basin, 1961.

ISE, L. International Submarine Engineering Ltd. Web Based AUV DesignInfo. BC, Canada, 2000. Disponível em http://www.ise.bc.ca.

KWAK, S.-S. Development of an Extensible Interactive Modeling, Simulation, Animation, and Real-Time Control (MoSART) Submarine Environment: A Tool for Enhancing Research and Education. 164 p. Dissertação (Master's Thesis) - Arizona State Univ., Arizona, USA, 1999.

LIN, C.-M.; MON, Y.-J. Fuzzy-logic-based guidance law design. In: IEEE Transactions on Aerospace and Electronic Systems. [S.l.]: IEEE, 2001.

LOGAN, C. L. Robust controller design and evaluation for a small underwater vehicle. 365 p. Dissertação (Master's Thesis) - Massachusetts Institute of Technology. Dept. of Electrical Engineering and Computer Science, Cambridge, MA, 1993.

LUNDSTRÖM, P.; SKOGESTAD, S.; DOYLE, J. Two-degree-of-freedom controller design for an ill-conditioned distillation process using $\mu$-synthesis. In: IEEE Transactions on Control Systems Technology. [S.1.]: IEEE Control Systems Society, 1999. v. 7, No 1, p. $12-21$.

MARTIN, R. J.; ATHANS, M.; VALVANI, L. S. Multivariable control of a submersible using the lqg/ltr design methodology. Laboratory for Information and Decision Systems, Massachusetts Institute of Technology, p. 12, 1986.

MCEWEN, R. Modeling and Control of a Variable-Length AUV. Moss Landing, CA 95039-9644, 2002.

MEIROVITCH, L. Introduction to dynamics and control. New York, USA: John Whiley \& Sons, 1985.

NAEEM, W.; SUTTON, R.; M.AHMAD, S. Lqg/ltr control of an autonomous underwater vehicle using a hybrid guidance law. In: Proceedings of GCUV'03 conference. Newport, UK: [s.n.], 2003. p. 35-40. 
NEWMAN, J. N. Marine Hydrodynamics. Massachusetts, USA: MIT Press, 1977.

PRESTERO, T. Verification of a Six-Degree Freedom simulation Model for the Remus Autonomous Underwater Vehicles. 128 p. Dissertação (Master's Thesis) - Massachusetts Institute of Technology and Wood Hole Oceanographic Institution, MA, CA-USA, 2001.

RENTSCHLER, M. E.; HOVER, F.; CHRYSSOSTOMIDIS, C. System identification of open-loop manoeuvring leads improved auv flight performance. IEEE Journal of Oceanic Engineering, v. 31, p. 200-1208, 2006.

RYOO, C.-K.; SHIN, H.-S.; TAHK, M.-J. Optimal waypoint guidance synthesis. In: Proceedings of the 44th IEEE Conference on Decision and Control, and the European Control Conference. Toronto, Canada: IEEE, 2005.

SBRAGIO, R. Projeto do Propulsor do Modelo-Livre - S3201-2450-RG-01. Brasil, 1995. Centro Tecnológico da Marinha em São Paulo, São Paulo, 1995.

SILVESTRE, C.; PASCOAL, A. Depth control of the infante auv using. gain-scheduled reduced-order output feedback. In: CAMS. Proc. of 16th IFAC World Congress. Prague, Czech Republic, 2005.

SKOGESTAD, S.; POSTLETHWAITE, I. Multivariable Feedback Control. West Sussex PO19 1UD, England: John Whiley \& Sons, 1996.

SONG, F.; AN, E.; SMITH, S. M. Design robust nonlinear controllers for autonomous underwater vehicles with comparison of simulated and at-sea test data. Journal of Vibration and Control, v. 8(2), p. 189-217, 2002.

SORANI, L. A. Estudo da dinâmica e controle de um submersivel não tripulado para uso no ambiente fluvial. 81 p. Dissertação (Mestrado) — Escola Politécnica, Universidade de São Paulo, São Paulo, 2002.

SOUZA, E. C. Modelagem e Controle de Veículos Submarinos Não Tripulados. 150 p. Dissertação (Mestrado) — Escola Politécnica, Universidade de São Paulo, São Paulo, 2003.

STRANG, G. Linear Algebra and its Aplications. N. York: Academic Press, 1988.

TANNER, O.; GEERING, H. P. Two-degree-of-freedom robust controller for an autonomous helicopter. In: Proceedings of the American Control Conference. Colorado, USA: IEEE, 2003. p. 993-998.

TRIANTAFYLlOU, M. S.; HOVER, F. S. Maneuvering and Control of Marine Vehicles. Cambridge, Massachusetts, USA: The M.I.T. PRESS, 2003.

VALAVANIS, K. P.; GRACANIN, D.; MATIJASEVIC, M.; KOLLURU, R.; DEMETRIOU, G. Control architectures for autonomous underwater vehicles. Control Systems Magazine, IEEE, v. 17, p. 48-64, 1997.

WHITCOMB, L. L. Underwater robotics: Out of the research laboratory and into the field. International conference in Robotics and Automation, IEEE, Preprint of an invited paper to appear in the Special Session on Industrial Robotics, p. 1-6, 2000. 
ZAMES, G. Optimal sensitivity and feedback; weighted seminorms, appoximate inverses, and plant invariant schemes. In: Proceedings 17th Allerton Conference. [S.l.: s.n.], 1979. p. $744-752$.

ZHOU, K. Essential of Robust Control. http://www.ee.lsu.edu/kemin/Courses.htm: Slides, Lecture Notes of Advanced Control Systems, EE 7510, 2000.

ZHOU, K.; DOYLE, J. C.; GLOVER, K. Robust and Optimal Control. [S.l.]: Prentice-Hall, 1995. 


\section{Apêndice A - Hidrodinâmica do VSA}

\section{A.1 Coeficientes Hidrodinâmicos do Veículo}

Tabela A.1: Parâmetros hidrodinâmicos normalizados para o VSA em 6 DOF

\begin{tabular}{|c|c|c|}
\hline $\begin{array}{l}X_{\delta_{r} \delta_{r}}^{\prime}=-0,32 \cdot 10^{-2} \\
X_{r r}^{\prime}=0,39 \cdot 10^{-3} \\
X_{v r}^{\prime}=0,19 \cdot 10^{-1}\end{array}$ & $\begin{array}{l}X_{\delta_{s} \delta_{s}}^{\prime}=-0,25 \cdot 10^{-2} \\
X_{u u}^{\prime}=-0,34 \cdot 10^{-3} \\
X_{w q}^{\prime}=-0,13 \cdot 10^{-1}\end{array}$ & $\begin{array}{l}X_{q q}^{\prime}=-0,23 \cdot 10^{-3} \\
X_{\dot{u}}^{\prime}=-0,10 \cdot 10^{-2}\end{array}$ \\
\hline $\begin{array}{l}Y_{\delta_{r}}^{\prime}=0,86 \cdot 10^{-2} \\
Y_{p p}^{\prime}=-0,24 \cdot 10^{-3} \\
Y_{\dot{r}}^{\prime}=0,11 \cdot 10^{-1} \\
Y_{v|v| R}^{\prime}=-0,11\end{array}$ & $\begin{array}{l}Y_{p}^{\prime}=-0,52 \cdot 10^{-3} \\
Y_{p q}^{\prime}=0,13 \cdot 10^{-1} \\
Y_{v}^{\prime}=-0,50 \cdot 10^{-1} \\
Y_{w p}^{\prime}=0,13 \cdot 10^{-1}\end{array}$ & $\begin{array}{l}Y_{\dot{\dot{p}}}^{\prime}=0,31 \cdot 10^{-2} \\
Y_{r}^{\prime}=-0,11 \cdot 10^{-3} \\
Y_{\dot{v}}^{\prime}=-0,23 \cdot 10^{-1}\end{array}$ \\
\hline $\begin{array}{l}Z_{\delta_{s}}^{\prime}=-0,81 \cdot 10^{-2} \\
Z_{v p}^{\prime}=-0,81 \cdot 10^{-1}\end{array}$ & $\begin{array}{l}Z_{q}^{\prime}=-0,92 \cdot 10^{-2} \\
Z_{w}^{\prime}=-0,17 \cdot 10^{-1}\end{array}$ & $\begin{array}{l}Z_{\dot{q}}^{\prime}=-0,57 \cdot 10^{-3} \\
Z_{\dot{w}}^{\prime}=-0,13 \cdot 10^{-1}\end{array}$ \\
\hline $\begin{array}{l}K_{\delta_{r}}^{\prime}=-0,11 \cdot 10^{-3} \\
K_{q r}^{\prime}=-0,11 \cdot 10^{-3} \\
K_{v}^{\prime}=-0,52 \cdot 10^{-3}\end{array}$ & $\begin{aligned} K_{p}^{\prime} & =-0,31 \cdot 10^{-3} \\
K_{r}^{\prime} & =-0,79 \cdot 10^{-5} \\
K_{\dot{v}}^{\prime} & =-0,31 \cdot 10^{-2}\end{aligned}$ & $\begin{array}{l}K_{\dot{p}}^{\prime}=-0,42 \cdot 10^{-4} \\
K_{\dot{r}}^{\prime}=-0,44 \cdot 10^{-3} \\
K_{w p}^{\prime}=0,11 \cdot 10^{-3}\end{array}$ \\
\hline $\begin{array}{l}M_{*}^{\prime}=-0,21 \cdot 10^{-4} \\
M_{\dot{q}}^{\prime}=-0,18 \cdot 10^{-3} \\
M_{\dot{w}}^{\prime}=0,37 \cdot 10^{-2}\end{array}$ & $\begin{array}{l}M_{\delta s}^{\prime}=-0,37 \cdot 10^{-2} \\
M_{r p}^{\prime}=0,98 \cdot 10^{-3} \\
M_{w|w| R}^{\prime}=-0,11 \cdot 10^{-1}\end{array}$ & $\begin{array}{l}M_{q}^{\prime}=-0,35 \cdot 10^{-2} \\
M_{w}^{\prime}=-0,57 \cdot 10^{-3}\end{array}$ \\
\hline $\begin{array}{l}N_{\delta_{r}}^{\prime}=-0,42 \cdot 10^{-2} \\
N_{p q}^{\prime}=-0,65 \cdot 10^{-3} \\
N_{v}^{\prime}=-0,11 \cdot 10^{-3}\end{array}$ & $\begin{array}{l}N_{p}^{\prime}=-0,83 \cdot 10^{-4} \\
N_{r}^{\prime}=-0,41 \cdot 10^{-2} \\
N_{\dot{v}}^{\prime}=-0,11 \cdot 10^{-1}\end{array}$ & $\begin{array}{l}N_{\dot{p}}^{\prime}=-0,79 \cdot 10^{-5} \\
N_{\dot{r}}^{\prime}=-0,11 \cdot 10^{-3} \\
N_{v|v| R}^{\prime}=-0,72 \cdot 10^{-2}\end{array}$ \\
\hline $\begin{array}{l}x_{G}=0,09 m \\
x_{B}=0,09 m \\
I_{x}=940 N-m \\
I_{x y}=0 N-m \\
c_{t}=0,16\end{array}$ & $\begin{array}{l}y_{G}=0 m \\
y_{B}=0 m \\
I_{y}=3631 N-m \\
I_{x z}=0 N-m \\
w_{e}=0,24\end{array}$ & $\begin{array}{l}z_{G}=0 m \\
z_{B}=-0,06 m \\
I_{z}=3631 N-m \\
I_{y z}=0 N-m\end{array}$ \\
\hline
\end{tabular}


Tabela A.2: Parâmetros hidrodinâmicos para o VSA em 6 DOF

\begin{tabular}{|c|c|c|}
\hline $\begin{array}{l}X_{\delta_{r} \delta_{r}}=\frac{\rho}{2} l^{2} X_{\delta_{r} \delta_{r}}^{\prime} \\
X_{r r}=\frac{\rho}{2} l^{4} X_{r r}^{\prime} \\
X_{v r}^{\prime}=\frac{\rho}{2} l^{3}\end{array}$ & $\begin{array}{l}X_{\delta_{s} \delta_{s}}=\frac{\rho}{2} l^{2} X_{\delta_{s} \delta_{s}}^{\prime} \\
X_{u u}=\frac{\rho}{2} l^{2} X_{u u}^{\prime} \\
X_{w q}^{\prime}=\frac{\rho}{2} l^{3}\end{array}$ & $\begin{array}{l}X_{q q}=\frac{\rho}{2} l^{4} X_{q q}^{\prime} \\
X_{\dot{u}}=\frac{\rho}{2} l^{3} X_{\dot{u}}^{\prime}\end{array}$ \\
\hline $\begin{array}{l}Y_{\delta_{r}}=\frac{\rho}{2} l^{2} Y_{\delta_{r}}^{\prime} \\
Y_{p|p|}=\frac{\rho}{2} l^{4} Y_{p|p|}^{\prime} \\
Y_{\dot{r}}=\frac{\rho}{2} l^{4} Y_{\dot{r}}^{\prime} \\
Y_{v|v| R}=\frac{\rho}{2} l^{2} Y_{v|v| R}^{\prime}\end{array}$ & $\begin{array}{l}Y_{p}=\frac{\rho}{2} l^{3} Y_{p}^{\prime} \\
Y_{p q}=\frac{\rho}{2} l^{4} Y_{p q}^{\prime} \\
Y_{v}=\frac{\rho}{2} l^{2} Y_{v}^{\prime} \\
Y_{w p}=\frac{\rho}{2} l^{3} Y_{w p}^{\prime}\end{array}$ & $\begin{aligned} Y_{\dot{p}} & =\frac{\rho}{2} l^{4} Y_{\dot{p}}^{\prime} \\
Y_{r} & =\frac{\rho}{2} l^{3} Y_{r}^{\prime} \\
Y_{\dot{v}} & =\frac{\rho}{2} l^{3} Y_{\dot{v}}^{\prime}\end{aligned}$ \\
\hline $\begin{aligned} Z_{\delta_{s}} & =\frac{\rho}{2} l^{2} Z_{\delta_{s}}^{\prime} \\
Z_{v p} & =\frac{\rho}{2} l^{3} Z_{v p}^{\prime}\end{aligned}$ & $\begin{aligned} Z_{q} & =\frac{\rho}{2} l^{3} Z_{q}^{\prime} \\
Z_{w} & =\frac{\rho}{2} l^{2} Z_{w}^{\prime}\end{aligned}$ & $\begin{aligned} Z_{\dot{q}} & =\frac{\rho}{2} l^{4} Z_{\dot{q}}^{\prime} \\
Z_{\dot{w}} & =\frac{\rho}{2} l^{2} Z_{\dot{w}}^{\prime}\end{aligned}$ \\
\hline $\begin{array}{l}K_{\delta_{r}}=\frac{\rho}{2} l^{3} K_{\delta_{r}}^{\prime} \\
K_{q r}=\frac{\rho}{2} l^{5} K_{q r}^{\prime} \\
K_{v}=\frac{\rho}{2} l^{3} K_{v}^{\prime}\end{array}$ & $\begin{aligned} K_{p} & =\frac{\rho}{2} l^{4} K_{p}^{\prime} \\
K_{r} & =\frac{\rho}{2} l^{4} K_{r}^{\prime} \\
K_{\dot{v}} & =\frac{\rho}{2} l^{4} K_{\dot{v}}^{\prime}\end{aligned}$ & $\begin{array}{l}K_{\dot{p}}=\frac{\rho}{2} l^{5} K_{\dot{p}}^{\prime} \\
K_{\dot{r}}=\frac{\rho}{2} l^{5} K_{\dot{r}}^{\prime} \\
K_{w p}=\frac{\rho}{2} l^{4} K_{w p}^{\prime}\end{array}$ \\
\hline $\begin{aligned} M_{*} & =\frac{\rho}{2} l^{3} M_{*}^{\prime} \\
M_{\dot{q}} & =\frac{\rho}{2} l^{5} M_{\dot{q}}^{\prime} \\
M_{\dot{w}} & =\frac{\rho}{2} l^{4} M_{\dot{w}}^{\prime}\end{aligned}$ & $\begin{array}{l}M_{\delta_{s}}=\frac{\rho}{2} l^{3} M_{\delta_{s}}^{\prime} \\
M_{r p}=\frac{\rho}{2} l^{5} M_{r p}^{\prime} \\
M_{w|w| R}=\frac{\rho}{2} l^{3} M_{w|w| R}^{\prime}\end{array}$ & $\begin{array}{l}M_{q}=\frac{\rho}{2} l^{4} M_{q}^{\prime} \\
M_{w}=\frac{\rho}{2} l^{3} M_{w}^{\prime}\end{array}$ \\
\hline $\begin{array}{l}N_{\delta_{r}}=\frac{\rho}{2} l^{3} N_{\delta_{r}}^{\prime} \\
N_{p q}=\frac{\rho}{2} l^{5} N_{p q}^{\prime} \\
N_{v}=\frac{\rho}{2} l^{3} N_{v}^{\prime}\end{array}$ & $\begin{array}{l}N_{p}=\frac{\rho}{2} l^{4} N_{p}^{\prime} \\
N_{r}=\frac{\rho}{2} l^{4} N_{r}^{\prime} \\
N_{\dot{v}}=\frac{\rho}{2} l^{4} N_{\dot{v}}^{\prime}\end{array}$ & $\begin{array}{l}N_{\dot{p}}=\frac{\rho}{2} l^{5} N_{\dot{p}}^{\prime} \\
N_{\dot{r}}=\frac{\rho}{2} l^{5} N_{\dot{r}}^{\prime} \\
N_{v|v| R}=\frac{\rho}{2} l^{3} N_{v|v| R}^{\prime}\end{array}$ \\
\hline
\end{tabular}




\section{A.2 Modelo Linear do VSA}

Nesta seção apresentam-se as matrizes utilizadas para o modelo VSA, linearizadas ao redor da velocidade de avanço de $u=2 \mathrm{~m} / \mathrm{s}$.

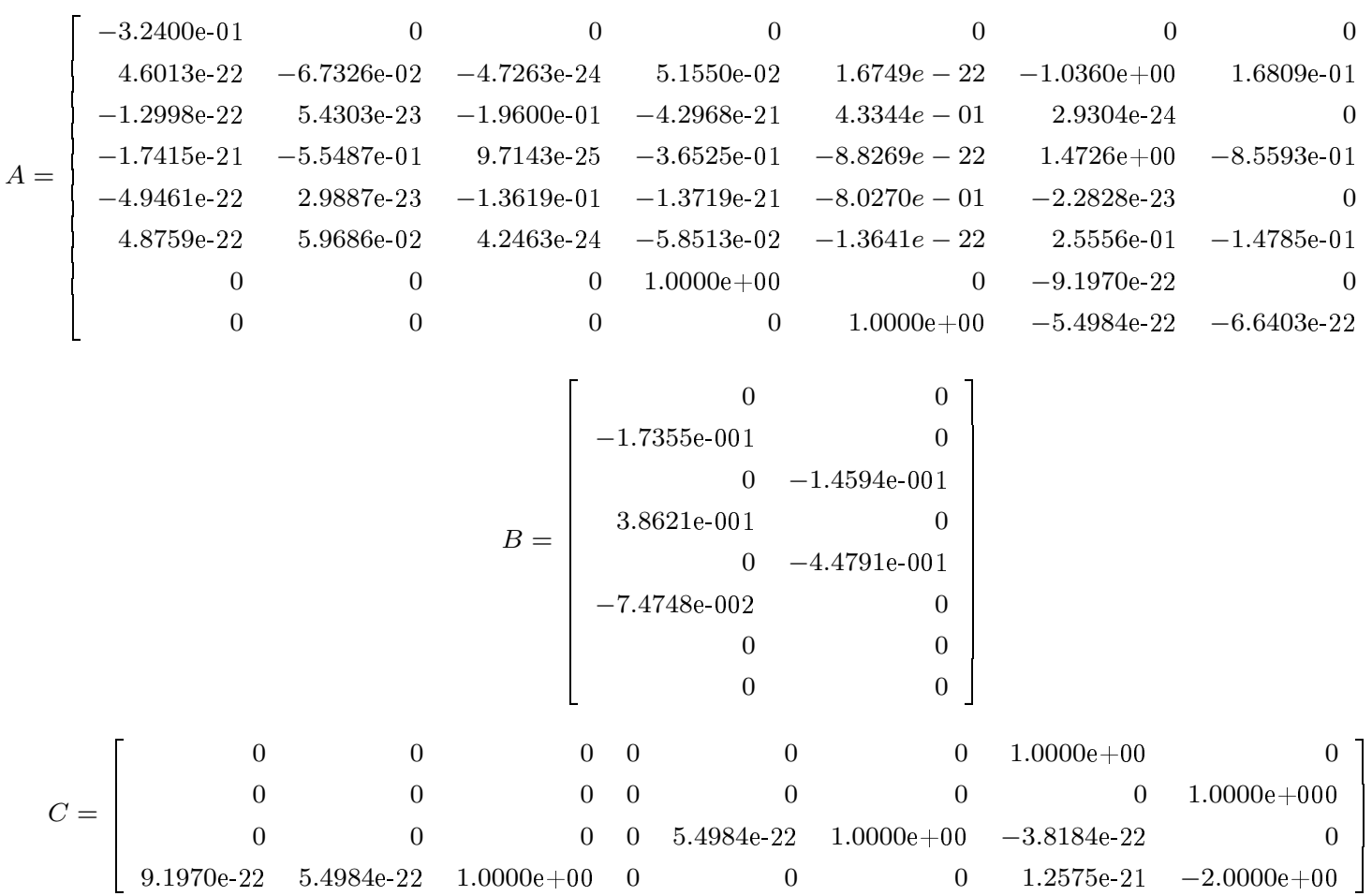

$$
\begin{aligned}
& D=\left[\begin{array}{ll}
0 & 0 \\
0 & 0 \\
0 & 0 \\
0 & 0
\end{array}\right]
\end{aligned}
$$

$\left.\begin{array}{r}0 \\ 0 \\ 2.3802 \mathrm{e}-02 \\ 0 \\ -1.8542 \mathrm{e}-01 \\ 0 \\ 6.6403 \mathrm{e}-22 \\ 0\end{array}\right]$

(A.1) 


\section{Apêndice B - Espaços Normados}

\section{B.1 Espaços $\mathcal{H}_{2}$ e $\mathcal{H}_{\infty}$}

Espaço $\mathcal{L}_{2}(j \mathbb{R})$ : todas as funções de matrizes complexas $F$ tal que a integral embaixo é limitada por:

$$
\int_{-\infty}^{\infty} \operatorname{Trace}\left[F^{*}(j \omega) F(j \omega)\right] d \omega<\infty
$$

com o produto interno

$$
\langle F, G\rangle:=\frac{1}{2 \pi} \int_{-\infty}^{\infty} \operatorname{Trace}\left[F^{*}(j \omega) G(j \omega)\right] d \omega
$$

e a norma induzida do produto interno é dado por:

$$
\|F\|_{2}:=\sqrt{\langle F, F\rangle}
$$

$\mathcal{R} \mathcal{L}_{2}(j \mathbb{R})$ ou simplesmente $\mathcal{R} \mathcal{L}_{2}$ : todas as matrizes de transferência estritamente próprias, reais e racionais sem pólos no eixo imaginário.

Espaço $\mathcal{H}_{2}$ : um subespaço (fechado) de $\mathcal{L}_{2}(j \mathbb{R})$ com funções $F(s)$ analíticas em $\operatorname{Re}(s)>$ 0 .

$$
\begin{aligned}
\|F\|_{2}^{2} & :=\sup _{\sigma>0}\left\{\frac{1}{2 \pi} \int_{-\infty}^{\infty} \operatorname{Trace}\left[F^{*}(\sigma+j \omega) F(\sigma+j \omega)\right] d \omega\right\} \\
& =\frac{1}{2 \pi} \int_{-\infty}^{\infty} \operatorname{Trace}\left[F^{*}(j \omega) F(j \omega)\right] d \omega
\end{aligned}
$$

$\mathcal{R H}_{2}$ (subespaço real racional de $\mathcal{H}_{2}$ ): todas as matrizes de transferência estáveis, estritamente próprias, reais e racionais.

Espaço $\mathcal{H}_{2}^{\perp}$ : o complemento ortogonal de $\mathcal{H}_{2}$ em $\mathcal{L}_{2}$ que são analíticas em $\operatorname{Re}(s)<0$. $\mathcal{R H}_{2}^{\perp}$ (o subespaço real e racional de $\mathcal{H}_{2}^{\perp}$ ): todas as matrizes de transferência anti-estáveis, estritamente próprias, reais e racionais. 
Relações de Parseval

$$
\begin{aligned}
\mathcal{L}_{2}(-\infty, \infty) & \cong \mathcal{L}_{2}(j \mathbb{R}) \quad \mathcal{L}_{2}[0, \infty) \cong \mathcal{H}_{2} \quad \mathcal{L}_{2}(-\infty, \infty] \cong \mathcal{H}_{2}^{\perp} \\
\|G\|_{2} & =\|g\|_{2} \quad \text { onde } G(s)=\mathcal{L}[g(t)] \in \mathcal{L}_{2}(j \mathbb{R})
\end{aligned}
$$

Espaço $\mathcal{L}_{\infty}(j \mathbb{R})$ : ou simplesmente $\mathcal{L}_{\infty}$, é o espaço Banach ou funções matriciais avaliadas, que são limitadas em $j \mathbb{R}$, com a norma

$$
\|F\|_{\infty}:=\operatorname{ess} \sup _{\omega \in \mathbb{R}} \bar{\sigma}[F(j \omega)] .
$$

$\mathcal{R} \mathcal{L}_{\infty}(j \mathbb{R})$ ou simplesmente $\mathcal{R} \mathcal{L}_{\infty}$ : todas as matrizes de transferência próprias, reais e racionais que não contem pólos no eixo imaginário.

Espaço $\mathcal{H}_{\infty}$ : é um subespaço (fechado) de $\mathcal{L}_{\infty}$ com funções que são analíticas e limitadas no semiplano direito. A norma $\mathcal{H}_{\infty}$ é definida como:

$$
\|F\|_{\infty}:=\sup _{\operatorname{Re}(s)>0} \bar{\sigma}[F(s)]=\sup _{\omega \in \mathbb{R}} \bar{\sigma}[F(j \omega)]
$$

A segunda igualdade pode ser considerada como uma generalização do teorema de modulo máximo para funções de transferência.

$\mathcal{R H}_{\infty}$ : todas as matrizes de transferência estáveis, próprias, reais e racionais.

Espaço $\mathcal{H}_{\infty}^{-}$: um subespaço (fechado) de $\mathcal{L}_{\infty}$ com funções que são analíticas e limitadas no semiplano esquerdo. A norma $\mathcal{H}_{\infty}^{-}$é definida

$$
\|F\|_{\infty}:=\sup _{\operatorname{Re}(s)<0} \bar{\sigma}[F(s)]=\sup _{\omega \in \mathbb{R}} \bar{\sigma}[F(s)]
$$

$\mathcal{R} \mathcal{H}_{\infty}^{-}$: todas as matrizes de transferência anti-estáveis, próprias, reais e racionais.

\section{B.2 Definições para Sistemas}

1.G(s) é estritamente própria $\Leftrightarrow \lim _{s \rightarrow \infty} G(s) \rightarrow 0$

2.G(s) é semi imprópria $\Leftrightarrow \lim _{s \rightarrow \infty} G(s) \rightarrow \alpha \neq 0$

3.se $G(s)$ é estritamente própria ou semi imprópria $\rightarrow G(s)$ é própria

4.G(s) é imprópria $\Leftrightarrow \lim _{s \rightarrow \infty} G(s) \rightarrow \infty$ 


\section{B.3 Transformação Fracional Linear $\boldsymbol{L F T}$}

As transformações fracionais lineares, servem para interconectar sistemas na representação de dois portos. Considere-se a planta generalizada $P$ de dimensão $\left(n_{1}+n_{2}\right) \times\left(m_{1}+m_{2}\right)$ e particionada como segue:

$$
P=\left[\begin{array}{ll}
P_{11} & P_{12} \\
P_{21} & P_{22}
\end{array}\right]
$$

Sejam $\triangle$ e $K$ de dimensão $m_{1} \times n_{1}$ e $m_{2} \times n_{2}$, respectivamente (compatível com a partição de $P$ ). Pode-se obter as transformações fracionais lineares $F_{u}$ e $F_{l}$, superior e inferior, respectivamente:

$$
\begin{aligned}
& F_{u}(P, \triangle) \triangleq P_{22}+P_{21} \triangle\left(I-P_{11} \triangle\right)^{-1} P_{12}=R \\
& F_{l}(P, K) \triangleq P_{11}+P_{12} K\left(I-P_{22} K\right)^{-1} P_{21}=N
\end{aligned}
$$

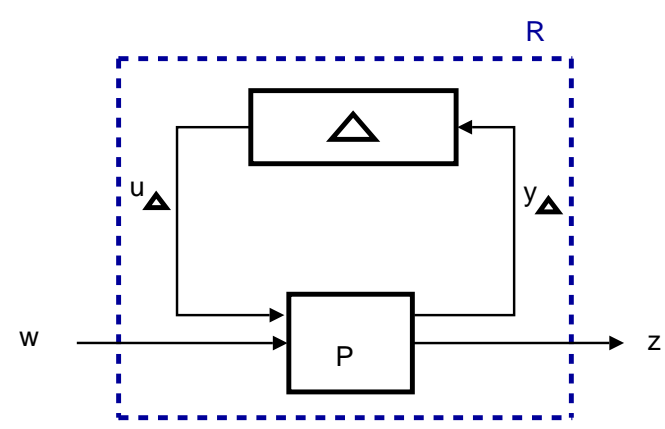

(a)

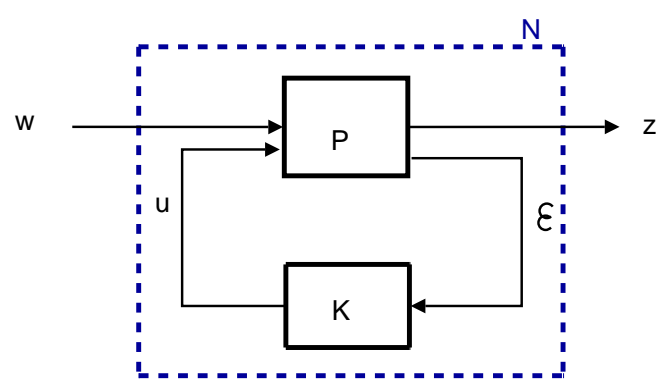

(b)

Figura B.1: Transformações Fracionais Lineares 


\section{Apêndice C - Métodos Numéricos}

Neste apêndice apresenta-se a solução numérica para a equação de evolução de estados do modelo (3.56), dado que o procedimento para a equação de saídas instantâneas é o mesmo.

\section{C.1 Método de Euler}

O método de Euler consiste na aplicação da fórmula iterativa:

$$
\mathbf{x}_{n+1}=\mathbf{x}_{n}+\mathbf{f}\left(\mathbf{x}_{n}, \mathbf{u}_{n}\right) \cdot \Delta t
$$

onde $\Delta t$ é o tempo de passo do modelo. Ou seja a cada instante de tempo cria-se uma nova informação para o vetor de estados, a partir do estado anterior e da atualização através das equações da dinâmica do sistema do sistema.

O método Euler que computacionalmente requer pequeno esforço, é inaceitável quando leva a soluções divergentes quando se utilizam intervalos de tempos muito grandes.

\section{C.2 Método de Euler modificado}

O método seguinte é uma modificação do método de Euler, onde dois coeficientes são introduzidos na fórmula a fim de conseguir maior precisão. Este método também é conhecido como integração trapezoidal, onde:

$$
\begin{aligned}
& \mathbf{k}_{1}=\mathbf{x}_{n}+\mathbf{f}\left(\mathbf{x}_{n}, \mathbf{u}_{n}\right) \cdot \Delta t \\
& \mathbf{k}_{2}=\mathbf{f}\left(\mathbf{k}_{1}, \mathbf{u}_{n+1}\right)
\end{aligned}
$$

A partir das equações anteriores obtemos:

$$
\mathbf{x}_{n+1}=\mathbf{x}_{n}+\frac{\Delta t}{2} \mathbf{f}\left(\left(\mathbf{x}_{n}, \mathbf{u}_{n}\right)+\mathbf{k}_{2}\right)
$$




\section{C.3 Método Runge Kutta}

Para minimizar o erro por aproximação, podem introduzir-se mais pontos intermédios (coeficientes). Por exemplo, utilizando quatro destes pontos:

$$
\begin{aligned}
& \mathbf{k}_{1}=\mathbf{x}_{n}+\mathbf{f}\left(\mathbf{x}_{n}, \mathbf{u}_{n}\right) \\
& \mathbf{k}_{2}=\mathbf{f}\left(\mathbf{x}+\frac{\Delta t}{2} \mathbf{k}_{1}, \mathbf{u}_{n+1 / 2}\right) \\
& \mathbf{k}_{3}=\mathbf{f}\left(\mathbf{x}+\frac{\Delta t}{2} \mathbf{k}_{2}, \mathbf{u}_{n+1 / 2}\right) \\
& \mathbf{k}_{4}=\mathbf{f}\left(\mathbf{x}+\Delta t \mathbf{k}_{3}, \mathbf{u}_{n+1}\right)
\end{aligned}
$$

Onde o vetor $\mathbf{u}_{n+1 / 2}$, se consegue por interpolação, conforme:

$$
\mathbf{u}_{n+1 / 2}=\frac{1}{2}\left(\mathbf{u}_{n}+\mathbf{u}_{n+1}\right)
$$

Este é o método mais utilizado na solução numérica de sistemas de EDOs e também será usado neste trabalho.

$$
\mathbf{x}_{n+1}=\mathbf{x}_{n}+\frac{\Delta t}{6}\left(\mathbf{k}_{1}+2 \mathbf{k}_{2}+2 \mathbf{k}_{3}+\mathbf{k}_{4}\right)
$$

\section{C.4 Simulação Computacional}

Neste trabalho, implementou-se uma aproximação numérica para determinar a solução do modelo desenvolvido para o VSA.

No apêndice D está o código do programa que realiza esta tarefa. O Matlab foi eleito como ferramenta computacional para este propósito. O programa calcula os esforços e momentos do modelo para cada instante do tempo em função da velocidade e atitude do veículo. Estes esforços e momentos determinam as acelerações relativamente ao referencial móvel e à taxa de alteração da posição relativamente ao referencial fixo. Essas acelerações são usadas para calcular as novas velocidades do veículo.

O modelo do VSA, utiliza duas variáveis de entradas:

- Condições Iniciais, que refere-se ao estado inicial do vetor de estados.

- Sinal de Controle, dado pela velocidade de rotação do propulsor e pela inclinação dos lemes atuadores. 


\section{Apêndice D - Código fonte}

A seguir, apresenta-se o código fonte dos programas principais que vão permitir reproduzir o trabalho.

Modelo Dinâmico do veiculo submersível autônomo vsa2.m

function $[x \operatorname{dot}]=\mathrm{vsa} 2(\mathrm{t}, \mathrm{x}, \operatorname{del} \mathrm{r}, \operatorname{del} \mathrm{s}, \mathrm{n})$

$\%$ function $[x d o t]=v s a 2(t, x, \operatorname{delr}, \operatorname{dels}, n)$

$\%$ vsa2.m Autor: Juan Luque

Date: $18-\mathrm{Jul}-2005$

$\%$ You can use ODE45 function in matlab for solve the AUV nonlinear model

$\%$ (Autonomous Underwater Vehicle, VSA in Portuguese)

$\%$ You can use vsa2_resp.m for time responses and numerical solve for VSA

$\%$

$\%$ The hydrodynamics coefficients can be found in Canhetti Dissertation $\%(1998)$

$\mathrm{m}=1460 ; \quad \%$ vehicle mass

wei=m*9.8; \%weight of the vehicle

buo=wei; $\%$ Buoyance

$c t=0.16$;

$\mathrm{d}=0.33 * 2 ; \quad \%$ diameter of VSA

ix $=940$;

iy $=3631$;

$i z=3631$;

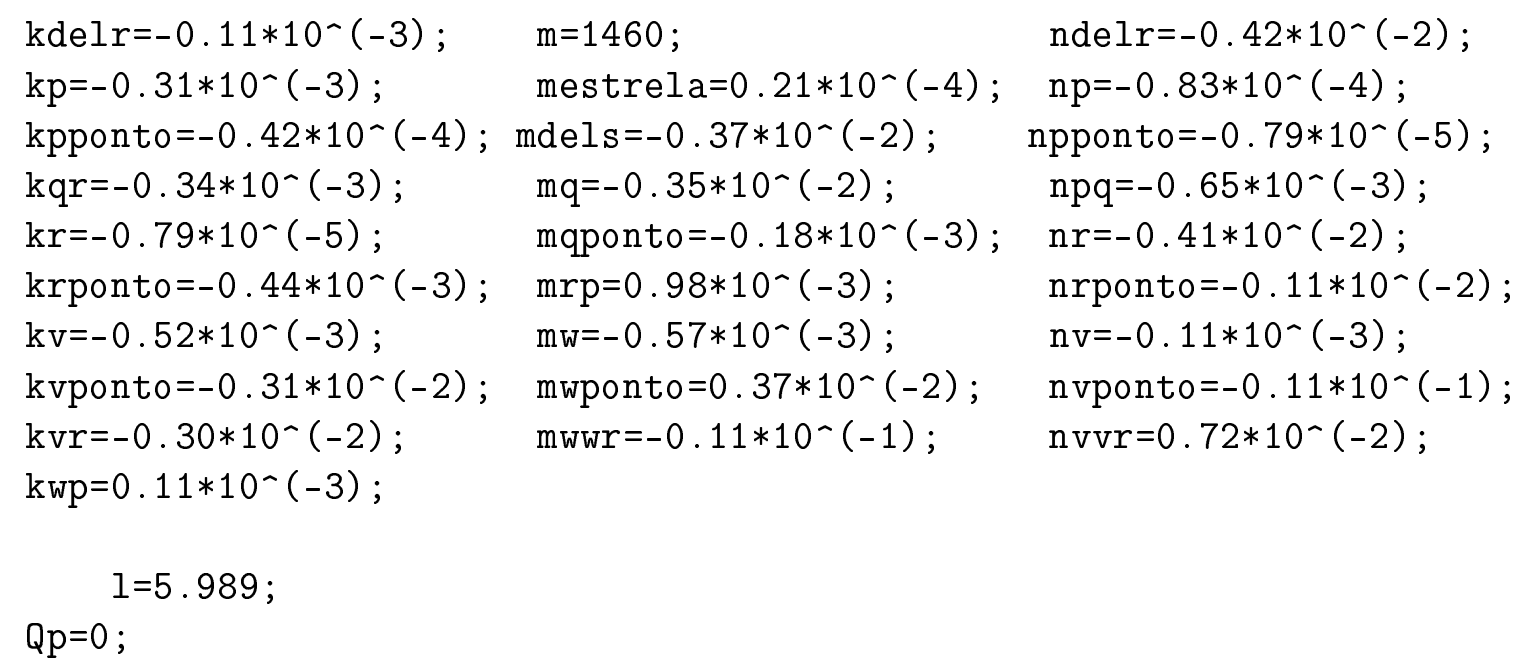


ro $=10^{-3}$;

we $=0.24$;

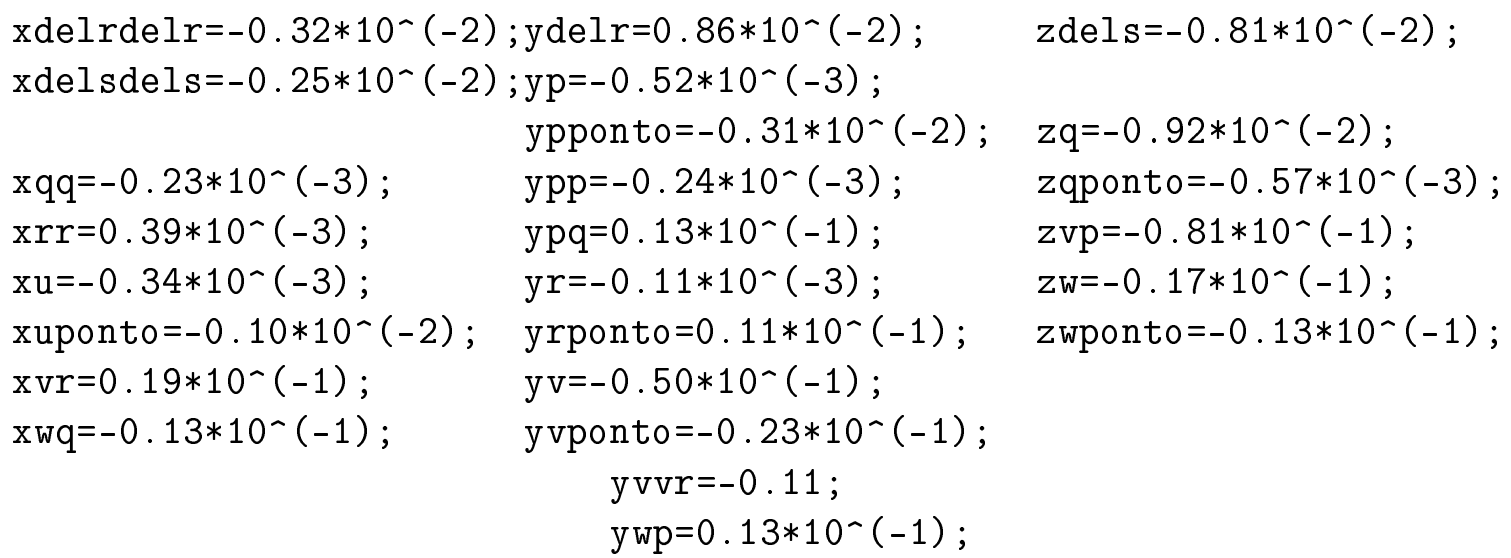


if teta $<=90 * \mathrm{deg}$

teta $=89.99 * \operatorname{deg}$

elseif teta $<=90.01 * \mathrm{deg}$

tet $a=90.01 * \operatorname{deg}$

else

teta=teta

end

end

$\%$

$\%$ Rudder and propeller

max_delr $=30 * \mathrm{pi} / 180$;

$\%$ max value delta_r (rad)

max_dels $=25 *$ pi $/ 180$;

$\%$ max value delta_s (rad)

max_n $\quad=15$;

$\% \max$ value $\mathrm{n}$ (rps)

$\%$ Rudder and shaft saturations

if abs(delr)>max_delr,delr=sign (delr)*max_delr;end

if abs (dels)>max_dels, dels=sign (dels)*max_dels; end

if abs(n)>max_n, delr=sign (n)*max_n; end

$\%$ Parameters, hydrodynamic derivatives and main dimensions

$c 1=\cos (f i)$;

$c 2=\cos ($ teta $)$;

c3 $=\cos (p s i)$;

$\mathrm{s} 1=\sin (\mathrm{fi})$;

s2 $=\sin ($ teta $)$;

s3 $=\sin ($ psi);

t2 $=\tan ($ teta $)$;

$\%$ Control input (rudder and propeller)

$\%$ delr = ui (1);

$\%$ dels = ui (2);

$\% \mathrm{n}=0 ; \% \mathrm{n}=2+0.2 * \mathrm{t}$;

$\%$

$\mathrm{j}=(\mathrm{u} *(1-\mathrm{we})) /(\mathrm{n} * \mathrm{~d})$;

$k t=-0.2482 * j \sim(2)-0.2272 * j+0.4823$;

$\mathrm{tp}=\mathrm{ro} * \mathrm{~d}^{\wedge}(4) * \mathrm{kt} * \mathrm{n}^{\wedge}(2)$;

if $\mathrm{n}<=0$

disp('cuidado $n$ negativo ou zero!')

end

$\mathrm{rv}=-0.2478 * \mathrm{u}^{\sim}(3)+24.986 * \mathrm{u}^{\sim}(2)+8.5217 * \mathrm{u}$;

$\%$

$r 2=(1 / 2) * r 0 * 1^{\wedge}(2) ; r 3=(1 / 2) * r o * 1^{\wedge}(3) ; r 4=(1 / 2) * r 0 * 1^{\wedge}(4) ; r 5=(1 / 2) * r 0 * 1^{\wedge}(5)$;

$\%$

MASS $=\ldots$

[m-r3*xuponto

0

0

0

$0 \quad \mathrm{~m}-\mathrm{r} 3 *$ yvponto $0 \quad-r 4 *$ ypponto $\quad 0 \quad-r 4 *$ yrponto 


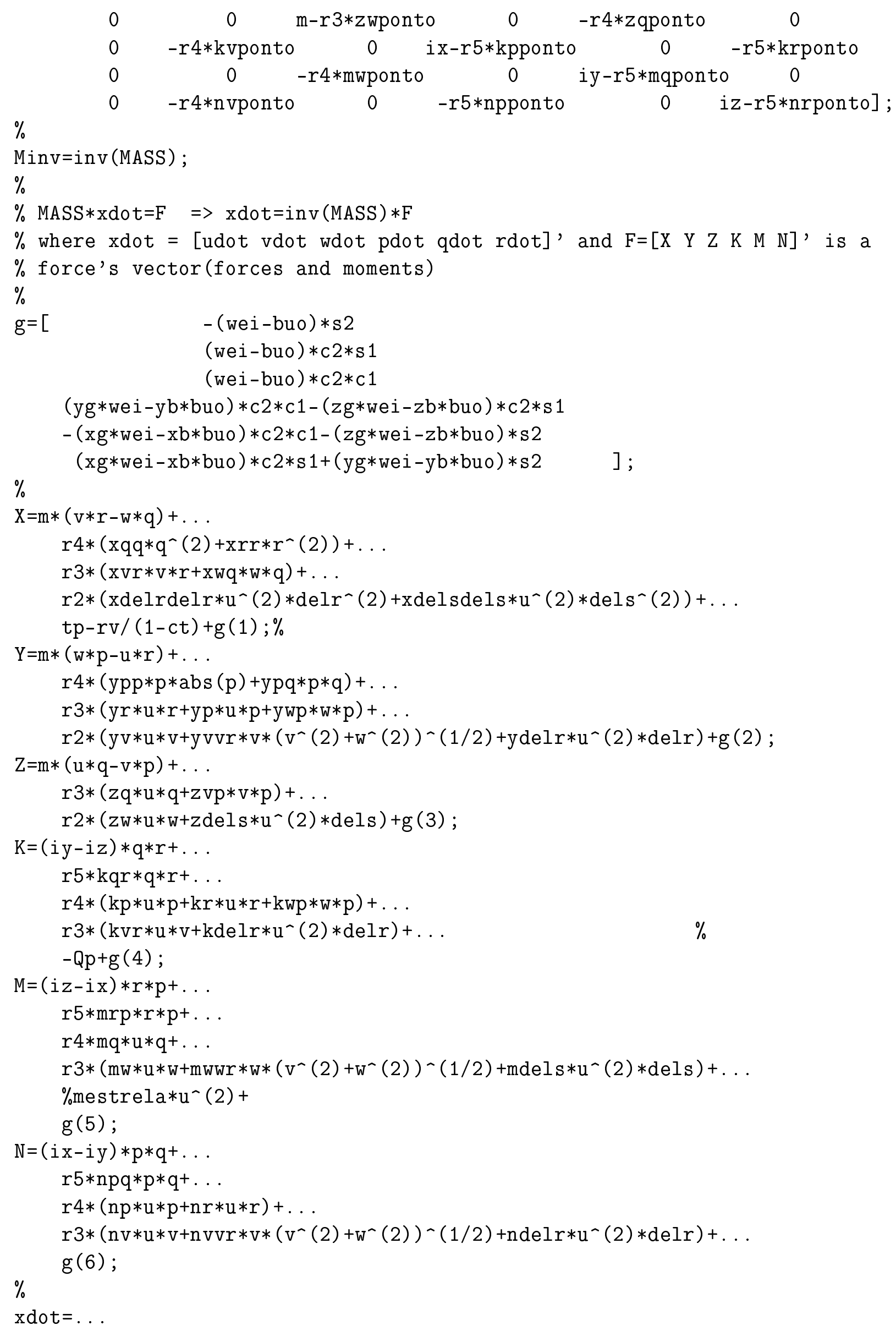


$[\operatorname{Minv}(1,1) * \mathrm{X}+\operatorname{Minv}(1,2) * \mathrm{Y}+\operatorname{Minv}(1,3) * \mathrm{Z}+\operatorname{Minv}(1,4) * \mathrm{~K}+\operatorname{Minv}(1,5) * \mathrm{M}+\operatorname{Minv}(1,6) * \mathrm{~N}$ $\operatorname{Minv}(2,1) * \mathrm{X}+\operatorname{Minv}(2,2) * \mathrm{Y}+\operatorname{Minv}(2,3) * \mathrm{Z}+\operatorname{Minv}(2,4) * \mathrm{~K}+\operatorname{Minv}(2,5) * \mathrm{M}+\operatorname{Minv}(2,6) * \mathrm{~N}$ $\operatorname{Minv}(3,1) * \mathrm{X}+\operatorname{Minv}(3,2) * \mathrm{Y}+\operatorname{Minv}(3,3) * \mathrm{Z}+\operatorname{Minv}(3,4) * \mathrm{~K}+\operatorname{Minv}(3,5) * \mathrm{M}+\operatorname{Minv}(3,6) * \mathrm{~N}$ $\operatorname{Minv}(4,1) * \mathrm{X}+\operatorname{Minv}(4,2) * \mathrm{Y}+\operatorname{Minv}(4,3) * \mathrm{Z}+\operatorname{Minv}(4,4) * \mathrm{~K}+\operatorname{Minv}(4,5) * \mathrm{M}+\operatorname{Minv}(4,6) * \mathrm{~N}$ $\operatorname{Minv}(5,1) * \mathrm{X}+\operatorname{Minv}(5,2) * \mathrm{Y}+\operatorname{Minv}(5,3) * \mathrm{Z}+\operatorname{Minv}(5,4) * \mathrm{~K}+\operatorname{Minv}(5,5) * \mathrm{M}+\operatorname{Minv}(5,6) * \mathrm{~N}$ $\operatorname{Minv}(6,1) * \mathrm{X}+\operatorname{Minv}(6,2) * \mathrm{Y}+\operatorname{Minv}(6,3) * \mathrm{Z}+\operatorname{Minv}(6,4) * \mathrm{~K}+\operatorname{Minv}(6,5) * \mathrm{M}+\operatorname{Minv}(6,6) * \mathrm{~N}$ $\mathrm{c} 3 * \mathrm{c} 2 * \mathrm{u}+(\mathrm{c} 3 * \mathrm{~s} 2 * \mathrm{~s} 1-\mathrm{s} 3 * \mathrm{c} 1) * \mathrm{v}+(\mathrm{s} 3 * \mathrm{~s} 1+\mathrm{c} 3 * \mathrm{c} 1 * \mathrm{~s} 2) * \mathrm{w}$ $\mathrm{s} 3 * \mathrm{c} 2 * \mathrm{u}+(\mathrm{c} 1 * \mathrm{c} 3+\mathrm{s} 1 * \mathrm{~s} 2 * \mathrm{~s} 3) * \mathrm{v}+(\mathrm{c} 1 * \mathrm{~s} 2 * \mathrm{~s} 3-\mathrm{c} 3 * \mathrm{~s} 1) * \mathrm{w}$ $-\mathrm{s} 2 * \mathrm{u}+\mathrm{c} 2 * \mathrm{~s} 1 * \mathrm{v}+\mathrm{c} 1 * \mathrm{c} 2 * \mathrm{w}$

$\mathrm{p}+\mathrm{s} 1 * \mathrm{t} 2 * \mathrm{q}+\mathrm{c} 1 * \mathrm{t} 2 * \mathrm{r}$

$(\mathrm{c} 1 * \mathrm{q}-\mathrm{s} 1 * \mathrm{r})$ $\mathrm{s} 1 / \mathrm{c} 2 * \mathrm{q}+\mathrm{c} 1 / \mathrm{c} 2 * \mathrm{r}$

Resposta Temporal para o VSA vsa2_resp.m

\% vsa2_resp.m

$\%$ Time Response for the vehicle 'VSA', for this purpose use vsa2.m file clear all

close all

$\mathrm{t}=[0: 0.1: 100]$;

$\mathrm{x} 0=\left[\begin{array}{llllllllllll}2 & 0 & 0 & 0 & 0 & 0 & 0 & 0 & 0 & 0 & 0 & 0\end{array}\right] ; \%$ initial conditions

$\%$ greater than $2.5 \mathrm{~m} / \mathrm{s}$ without control,

$\%$ the system go to instability

$\mathrm{ui}=\left[\begin{array}{lll}15 * \mathrm{pi} / 180 & 0 & 1 \mathrm{e}-6\end{array}\right]$;

$\%$ values for the atuator [delr dels n]

delr=ui (1);

$\%$ rudder, stern and propeller velocity

dels=ui (2);

$\mathrm{n}=\mathrm{ui}(3)$;

$f=18 ; \quad \%$ fontsize axes

$\%$ gcf for figures

$\%$ gca for axes

$[t, y]=0 d e 45($ Qvsa2 $, t, x 0,[], \operatorname{del} r, \operatorname{del} s, n) ; \%$ numerical solve for vsa.m using $\%$ 4ta Runge Kutta algorithm

set (0, 'DefaultTextInterpreter', 'latex')

figure (1)

$\%$ set(gcf,'position', [270 170560500$]$ ) \% position of the figure

set (gca,'fontsize',f); $\quad \%$ fontsize of the axes

$\%$ set (gca, 'position', [ [0.13 $0.583837 \quad 0.840 .341163]$ );

plot (t,y (:,1), 'k-', 'LineWidth',1); hold on;

plot $(t, y(:, 2)$, 'k--' , 'LineWidth', 2$)$;

plot $\left(t, y(:, 3),{ }^{\prime} \mathrm{k}:\right.$ ', 'LineWidth', 2$)$;

legend ('\$u \$', ' $\$ \mathrm{v} \$$ ', ' $\$ \mathrm{w} \$$ ') ; set (legend, 'Interpreter', 'latex');

title('Translation velocity')

xlabel ('time (s)', 'Interpreter', 'tex')

ylabel ('[u v w] (m/s)', 'Interpreter', 'tex')

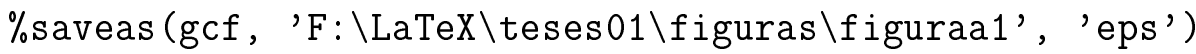

figure (2)

set (gca, 'fontsize',f);

plot (t,y $(:, 4),{ }^{\prime}-^{\prime}$, 'LineWidth', 1); hold on; 


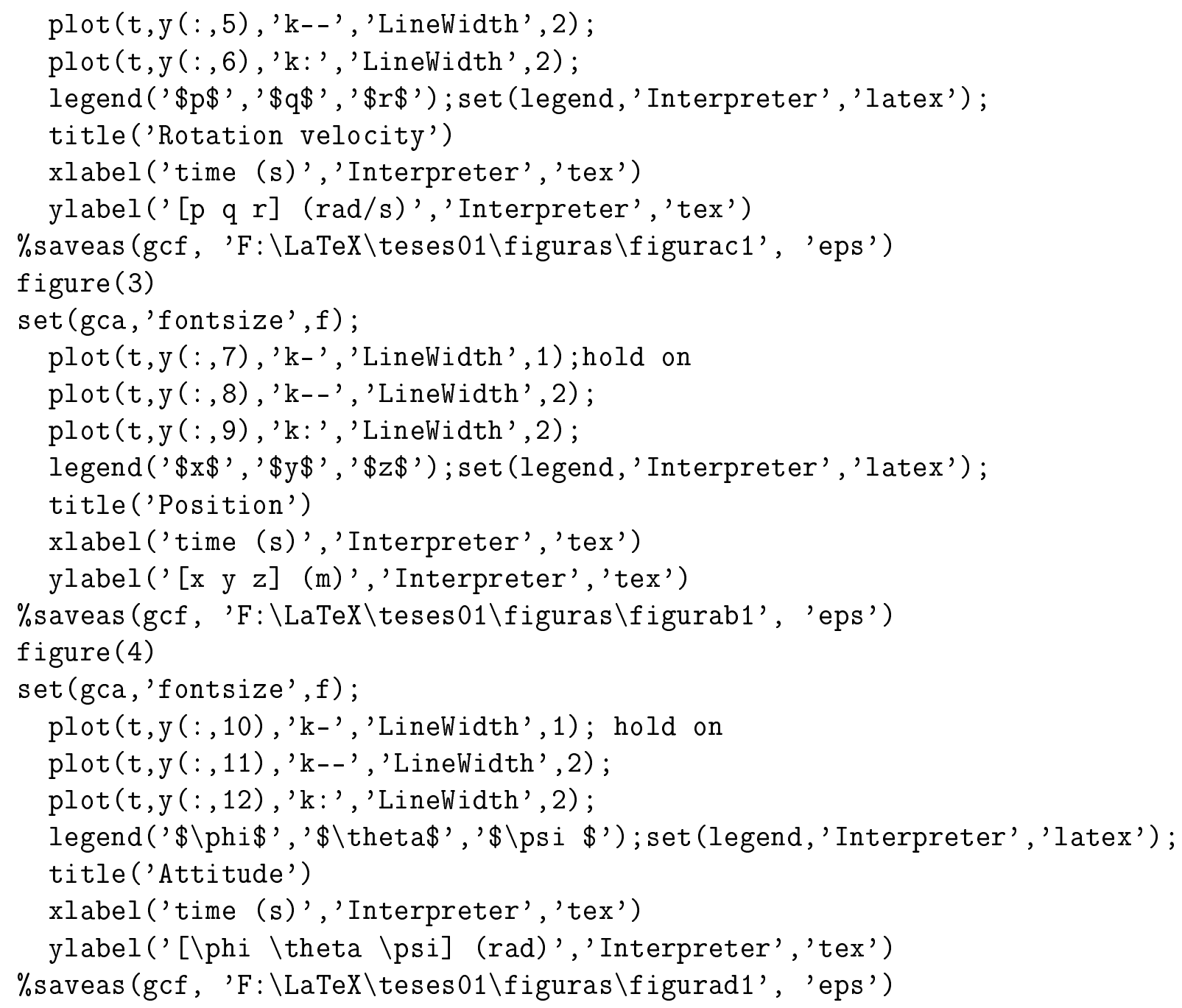

\section{D.1 Síntese do Controlador}

Síntese Do Controlador VSA vsa2_resp.m

$\%$ vsa2_hinf01

$\%$ Mixed Sensitivity for VSA, using $\mathrm{S}$ KS and $\mathrm{T}$ functions

$\%$ it needs the next programs for running correctly:

$\%$ vsa2_sfcn02.m, vsa2_sys02.mdl and vsa2.m vsa_weigth.m trimlin02.m

$\%$ 1) Get the linear model 12 states

$\%$ 2) Reduce the VSA-LTI model in 8 states, neglected states with no $\%$ dynamics effect

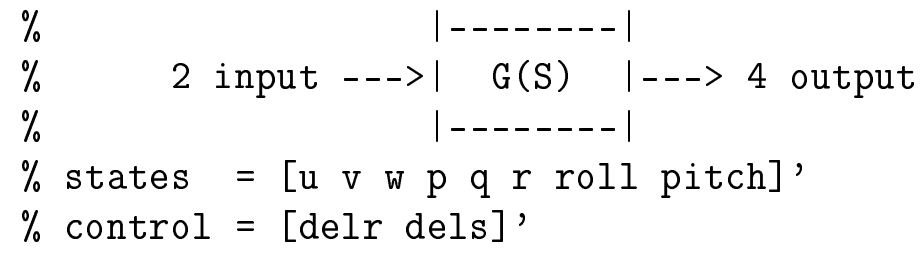


$\%$ output = [roll pitch yaw_rate depth_rate]'

$\% 3$ ) Scaling the VSA-LTI system

$\%$ 4) Select the weigthing functions

$\%$ 4) Put the system in correctly configuration for advanced control, i.e.

$\%$ extended Plant using sysic.

$\%$ 5) Synthesis of Controller using $\mathrm{H}_{\text {_infinity. }}$

$\%$ 6) Plot the responses (frequency and time)

$\%$

load trimlin02

$a p=a ; b p=b ; c p=c ; d p=d$;

$[a p, b p, c p, d p]=r e d u c i r(a p, b p, c p, d p) ; \%$ neglected variables with no dymamics $\%$ effect

$\%$ [ $\begin{array}{llll}x & y & z & p s i\end{array}$,

pzmap (ap, bp , cp, dp) ;

pause

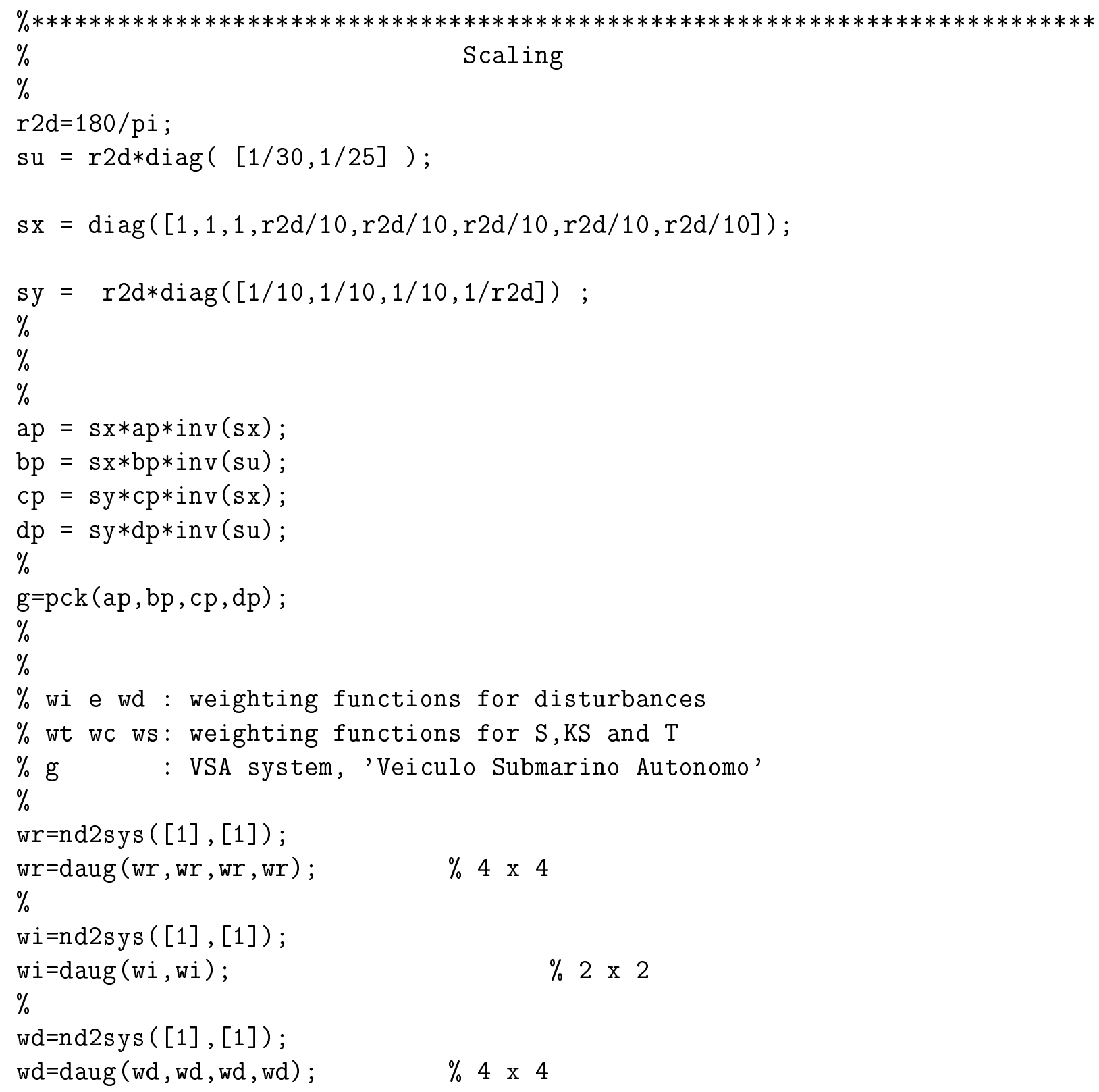




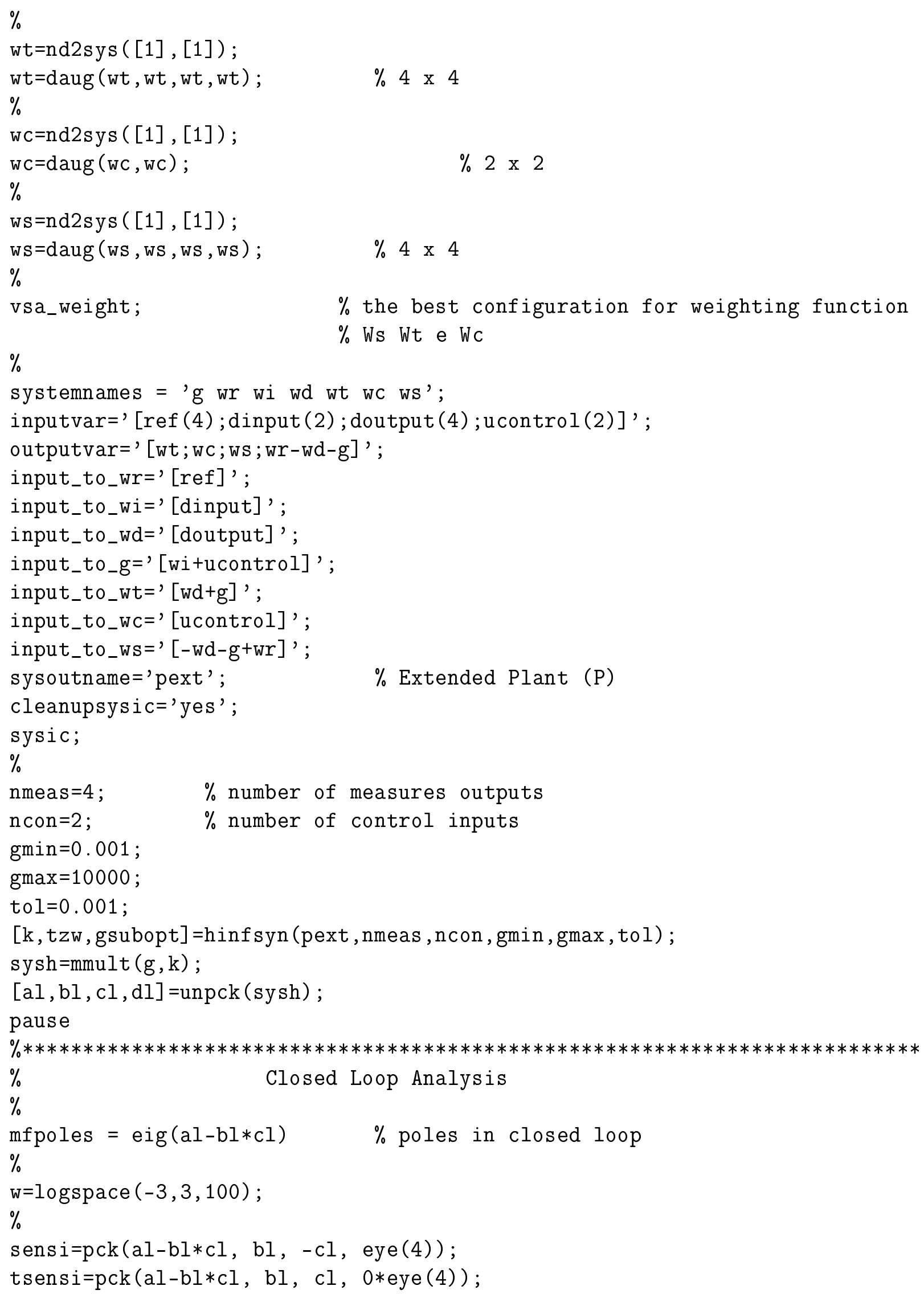

